\title{
Boundary Element Approximation for Maxwell's Eigenvalue Problem
}

\author{
Zur Erlangung des akademischen Grades eines \\ DOKTORS DER NATURWISSENSCHAFTEN
}

\author{
von der Fakultät für Mathematik des \\ Karlsruher Institut für Technologie \\ genehmigte \\ DISSERTATION
}

von

M. Sc. Jiping Xin

aus Göteborg, Sweden

Tag der mündlichen prüfung: 13.07.2011

Referent: Prof. Dr. Christian Wieners

Korreferent: Prof. Dr. Willy Dörfler 


\section{Contents}

Abstract

List of Figures $\quad$ v

List of Tables $\quad$ v

1 Boundary Element Methods for Boundary Value Problems 1

1.1 Classical electrodynamics . . . . . . . . . . . . . . . 3

1.2 The Helmholtz case . . . . . . . . . . . . . . . . . . 6

1.2.1 Representation formula . . . . . . . . . . . . . . 7

1.2.2 Function spaces . . . . . . . . . . . . . . . 7

1.2.3 Boundary integral equations . . . . . . . . . . . . . . 9

1.2.4 Variational formulations . . . . . . . . . . . . . . 12

1.2 .5 Galerkin-BEMs . . . . . . . . . . . . . . . 13

1.2 .6 Numerical tests . . . . . . . . . . . . . . . . . 17

1.3 The Maxwell case . . . . . . . . . . . . . . . . . . . . . . . . . . . . . . . . . . . 19

1.3.1 Representation formula . . . . . . . . . . . . . . . . . . 19

1.3.2 Function spaces . . . . . . . . . . . . . . . . 21

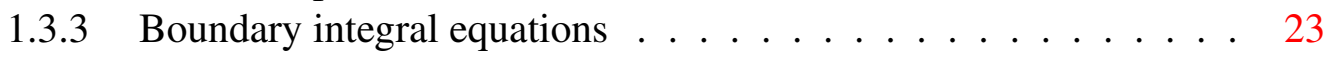

1.3.4 Variational formulations . . . . . . . . . . . . 27

1.3.5 Galerkin-BEMs . . . . . . . . . . . . . . . . . . . . . 29

1.3.6 Numerical tests . . . . . . . . . . . . . . 32

2 Domain Decomposition Methods

2.1 The Helmholtz case . . . . . . . . . . . . . . . . . . . . . . . . 34

2.1.1 Interface problem . . . . . . . . . . . . . . 34

2.1.2 Domain decomposition method .............. 35

2.1.3 Variational formulation . . . . . . . . . . . . . 36

2.1 .4 Galerkin-BEM . . . . . . . . . . . . . . . 37

2.1.5 Numerical tests . . . . . . . . . . . . . . . . . 38

2.2 The Maxwell case . . . . . . . . . . . . . . . . . . . . . . . . . 40

2.2.1 Interface problem . . . . . . . . . . . . . . . 40

2.2.2 Domain decomposition method . . . . . . . . . . . . . . 40

2.2.3 Variational formulation . . . . . . . . . . . . . . . . . 41

2.2 .4 Galerkin-BEM . . . . . . . . . . . . . . . . 43

2.2 .5 Numerical tests . . . . . . . . . . . . . . . . 45 
3 Boundary Element Methods for Eigenvalue Problems 47

3.1 A Priori error estimates for holomorphic eigenvalue problems . . . . . . . 47

3.1.1 Basic definitions . . . . . . . . . . . . . . . 47

3.1 .2 Convergence . . . . . . . . . . . . . . . . . 49

3.1 .3 A Priori error estimates . . . . . . . . . . . . . . . 50

3.2 The Helmholtz case . . . . . . . . . . . . . . . . . . . . 54

3.2.1 Nonlinear solution method for eigenvalue problem . . . . . . . . 54

3.2 .2 A Priori error estimates . . . . . . . . . . . . . . 55

3.2 .3 Numerical tests . . . . . . . . . . . . . . . . 57

3.3 The Maxwell case . . . . . . . . . . . . . . . . . . . . . . . . 59

3.3.1 Nonlinear solution method for eigenvalue problem . . . . . . . 59

3.3.2 A Priori error estimates . . . . . . . . . . . . . . . 60

3.3 .3 Numerical tests . . . . . . . . . . . . . . . 61

4 Boundary Element Methods for Interface Eigenvalue Problems 63

4.1 The Helmholtz case . . . . . . . . . . . . . . . . . . . . . . . 63

4.1.1 Nonlinear solution method for interface eigenvalue problem . . . 63

4.1.2 Numerical tests . . . . . . . . . . . . . . . . 66

4.2 The Maxwell case . . . . . . . . . . . . . . . . . . . . . . 69

4.2.1 Nonlinear solution method for interface eigenvalue problem . . . 69

4.2.2 Numerical tests . . . . . . . . . . . . . . . . . . 72

5 Comparison of BEMs and FEMs in Band Structure Computation in 3D $\begin{array}{ll}\text { Photonic Crystals } & 75\end{array}$

5.1 A brief introduction to photonic crystals . . . . . . . . . . . . 75

5.2 A homogeneous problem with periodic boundary conditions . . . . . . 78

5.2.1 Nonlinear solution method . . . . . . . . . . . . . 79

5.2 .2 Numerical tests . . . . . . . . . . . . . . . . . 80

5.3 An inhomogeneous problem with periodic boundary conditions . . . . . . 82

5.3.1 Nonlinear solution method . . . . . . . . . . . . . . 82

5.3 .2 Numerical tests . . . . . . . . . . . . . . . . 85

5.4 Comparison of BEMs and FEMs . . . . . . . . . . . . . . . . . . 87

5.4.1 Numerical tests . . . . . . . . . . . . . . . . . . . 88

$5.4 .2 \quad$ Numerical examples . . . . . . . . . . . . . . . . . 90

Bibliography $\quad 92$ 


\section{Abstract}

The aim of this thesis is to use Galerkin boundary element methods to solve the eigenvalue problems for the Helmholtz equation and the Maxwell's equations with an application to the computation of band structures of photonic crystals. Boundary element methods (BEM) may be considered as the application of Galerkin methods to boundary integral equations. The central to boundary element methods is the reduction of boundary value problems to equivalent boundary integral equations. This boundary reduction has the advantage of reducing the number of space dimension by one and the capability to solve problems involving infinite domains. The strategy for studying boundary integral equations by weak solutions is the same with partial differential equations. Boundary element methods are based on variational formulations and the strategy for studying boundary element methods is also the same with finite element methods. In Chapter 1 we give a brief introduction of Galerkin-BEMs for the Laplace and Helmholtz equations, and the Maxwell's equations for the Dirichlet and Neumann boundary value problems with a Priori error estimates. In Chapter 2 we use Galerkin-BEMs with domain decomposition methods to solve the inhomogeneous problems for the Helmholtz equation and the Maxwell's equations with a Priori error estimates. The numerical results confirm the a Priori results for boundary value problems. To solve eigenvalue problems by using boundary element methods is a new work. In Chapter 3 we give an introduction of Galerkin-BEMs for solving the eigenvalue problems for the Helmholtz equation and the Maxwell's equations with a Priori error estimates (three times). The proof of a Priori error estimates follow the Ph.D. work of Dr. Gerhard Unger in 2010. In Chapter 4 we use Galerkin-BEMs to solve the interface eigenvalue problems for the Helmholtz equation and the Maxwell's equations. The numerical results confirm the a Priori results. If we use Galerkin-BEMs to solve these eigenvalue problems, the linear eigenvalue problems will be changed to the nonlinear eigenvalue problems and we use the Newton method to solve this kind of nonlinear eigenvalue problems. Because of the limit of the Newton method, an alternative method such as the contour integral method will be considered in the further work after this thesis.

Photonic crystals are the materials which are composed of periodic dielectric or metallo-dielectric nanostructures. They exist in nature and have been studied for more than one hundred years. Photonic crystals can also be technically designed and produced to allow and forbid electromagnetic waves in a similar way that the periodicity of semiconductor crystals affects the motion of electrons. Since photonic crystals affect electromagnetic waves, the Maxwell's equations are used to describe this phenomena. When we design photonic crystals, we need to know for which frequencies electromagnetic waves can not propagate in them. So we need to calculate the frequencies 
and this is an eigenvalue problem. By using the famous Bloch theorem, the problem is changed from the whole domain to one unit cell with quasi-periodic boundary conditions. As a summary, we get an interface eigenvalue problem with quasi-periodic boundary conditions for the Maxwell's equations. In Chapter 5 we solve the eigenvalue problems in homogeneous and inhomogeneous mediums, respectively, with periodic boundary conditions. At the end we solve an interface eigenvalue problem with quasi-periodic boundary conditions as an example for the computation of band structures of photonic crystals and compare our results with finite element methods. The results from GalerkinBEMs match the results from finite element methods very well and we confirm the application of Galerkin-BEMs for solving this kind of eigenvalue problems. 


\section{List of Figures}

1.1 A flow chart of Galerkin-BEMs for boundary value problems . . . . . . . 2

1.2 Dirichlet boundary value problems for the Laplace and Helmholtz equations 18

1.3 Neumann boundary value problems for the Laplace and Helmholtz equations ........................... 19

1.4 Dirichlet and Neumann boundary value problems for Maxwell's equations 33

2.1 Interface problem with Dirichlet boundary condition for the Helmholtz equation . . . . . . . . . . . . . . . . . . . 39

2.2 Interface problem with Dirichlet boundary condition for Maxwell's equations ............................. 45

3.1 First eigenvector and second eigenvector of the Laplace eigenvalue problem with homogeneous Dirichlet boundary condition . . . . . . . 58

3.2 First eigenvector and second eigenvector of Maxwell eigenvalue problem with homogeneous Dirichlet boundary condition . . . . . . . . . . 62

4.1 First eigenvector of the interface eigenvalue problem for the Helmholtz equation with homogeneous Dirichlet boundary condition . . . . . . . 67

4.2 Second eigenvector of the interface eigenvalue problem for the Helmholtz equation with homogeneous Dirichlet boundary condition . . . . . . . 68

4.3 First eigenvector of the interface eigenvalue problem for Maxwell's equations with homogeneous Dirichlet boundary condition . . . . . . 73

4.4 Second eigenvector of the interface eigenvalue problem for Maxwell's equations with homogeneous Dirichlet boundary condition . . . . . . . 74

5.1 A simple definition of crystals . . . . . . . . . . . . . 76

$5.21 \mathrm{D}, 2 \mathrm{D}$ and 3D periodic structures of photonic crystals $\ldots \ldots . \ldots 76$

5.3 First eigenvector and second eigenvector of the eigenvalue problem for Maxwell's equations with periodic boundary conditions . . . . . . . . . 81

5.4 First eigenvector of the interface eigenvalue problem for Maxwell's equations with periodic boundary conditions . . . . . . . . . 86

5.5 Second eigenvector of the interface eigenvalue problem for Maxwell's equations with periodic boundary conditions . . . . . . . . . . . 87

5.6 Band structure of a homogeneous problem calculated by Galerkin-BEMs $\quad 89$

5.7 Band structure of an inhomogeneous problem solved by Galerkin-BEMs and FEMs . . . . . . . . . . . . . . . . . . . . . 90 


\section{List of Tables}

1.1 Accuracy of Galerkin-BEMs for Dirichlet boundary value problems for the Laplace and Helmholtz equations . . . . . . . . . . . . . . . . 18

1.2 Accuracy of Galerkin-BEMs for Neumann boundary value problems for the Laplace and Helmholtz equations . . . . . . . . . . . . . . . . . 18

1.3 Accuracy of Galerkin-BEMs for Dirichlet and Neumann boundary value problems for Maxwell's equations . . . . . . . . . . . . . 32

2.1 Accuracy of Galerkin-BEMs for interface problem with Dirichlet boundary condition for the Helmholtz equation . . . . . . . . . . . . . . . . 39

2.2 Accuracy of Galerkin-BEMs for interface problem with Dirichlet boundary condition for Maxwell's equations . . . . . . . . . . . . 46

3.1 Convergence of the first eigenvalue of the Laplace eigenvalue problem with homogeneous Dirichlet boundary condition . . . . . . . . . . . 57

3.2 Convergence of the second eigenvalue of the Laplace eigenvalue problem with homogeneous Dirichlet boundary condition . . . . . . . . . . . . 59

3.3 Convergence of the first eigenvalue and second eigenvalue of Maxwell eigenvalue problem with homogeneous Dirichlet boundary values . . . .

4.1 Convergence of the first eigenvalue and second eigenvalue of the interface eigenvalue problem for the Laplace equation with homogeneous Dirichlet boundary condition . . . . . . . . . . . . . . .

4.2 Convergence of the first eigenvalue and second eigenvalue of the interface eigenvalue problem for Maxwell's equations with homogeneous Dirichlet boundary condition . . . . . . . . . . . . . .

5.1 Convergence of the first eigenvalue and second eigenvalue of the eigenvalue problem for Maxwell's equations with periodic boundary conditions

5.2 Convergence of the first eigenvalue and second eigenvalue of the interface eigenvalue problem for Maxwell's equations with periodic boundary conditions . . . . . . . . . . . . . . . . . .

5.3 Convergence of the eigenvalues calculated by Galerkin-BEMs in band structure . . . . . . . . . . . . . . . . . .

5.4 Convergence of the eigenvalues calculated by finite element methods in band structure . . . . . . . . . . . . . . . . . . 


\section{Chapter 1}

\section{Boundary Element Methods for Boundary Value Problems}

Partial differential equations (PDE) and boundary integral equations (BIE) are used to describe different problems in physics and other research fields. At first we should have an understanding of a well-posed problem. A well-posed problem means the existence, uniqueness and stability of the solution. The study of these properties is the main work for PDEs and BIEs and we have two ways. One way is to find a representation formula for the solution. This kind of the solution is called a classical solution and the study could follow [27, 39, 25, 23]. A classical solution is usually required to be $k$-times continuously differentiable according to the order of the PDE. This is a strong condition and many boundary value problems don't have so regular solutions. Even if the solution is regular, it is also difficult to find a formula for it in many cases. So if we want to discuss a more general problem, we use the other way which generalizes the problem and discusses the properties of the solution by a variational formulation. This kind of the solution is called a weak solution and the study could follow [23, 5, 25]. The strategy for studying BIEs by a weak solution is exactly the same with PDEs [65, 62, 35]. Finite element methods (FEM) and boundary element methods (BEM) are based on variational formulations. The study of FEMs could follow [22, 47, 4, 50, 16]. As a summary we have three steps.

(1a) a generalization of the problem;

(1b) the existence, uniqueness and stability of a weak solution;

(1c) FEMs or BEMs based on variational formulations.

The main idea of (1a) for BIEs is to extend continuously differentiable function spaces to Sobolev spaces and operators are also extended to Sobolev spaces. The study of Sobolev spaces could follow $[26,60,1]$. Since Sobolev spaces and generalized operators are defined in a distributional sense, the continuously differentiable condition is released and the problem could be defined on a domain with a Lipschitz boundary. We have three steps for (1a) and five sub-steps for the continuity of boundary integral operators (BIO).

(2a) definitions of Sobolev spaces;

(2b) definitions of generalized operators; 
(2c) continuity of generalized operators.

- continuity of Neumann and Dirichlet trace operators;

- continuity of potential operators;

- potentials as weak solutions of a generalized problem;

- continuity of boundary integral operators;

- representations of singular integrals.

The next step (1b) is to define a variational formulation by a dual pairing and discuss the existence, uniqueness and stability of a weak solution. The Lax-Milgram theorem and Fredholm alternative lemma are the common tools used in this step. They need the bilinear form in the variational formulation to be elliptic or satisfy the Gårding inequality. This step need the knowledge of function analysis and the study could follow [20, 59, 5]. In the last step (1c) we need to define a boundary element space instead of the Sobolev space in the variational formulation and get a discretization formulation. The strategy to do the a Priori error estimates for BEMs is exactly the same with FEMs. They are the Cea's lemma, optimal convergence and super convergence. The study of BEMs could follow $[34,58,65,62]$

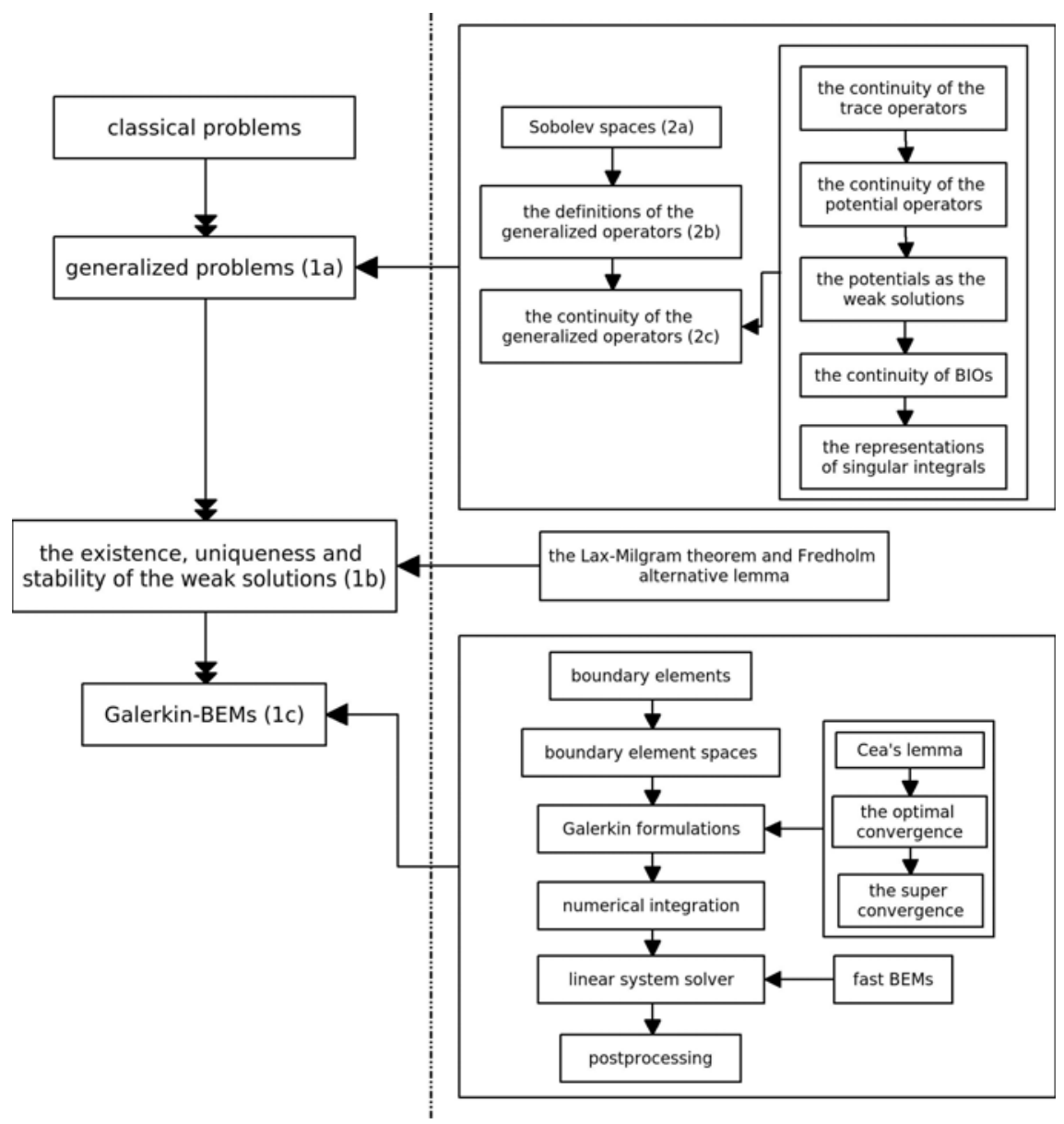

Figure 1.1 A flow chart of Galerkin-BEMs for boundary value problems 
Fig 1.1 is a flow chart of a standard procedure of the study of BIEs and BEMs for boundary value problems. In this chapter we follow Fig 1.1 to give an introduction of Galerkin-BEMs for Dirichlet and Neumann boundary value problems for the Helmholtz equation and the Maxwell's equations with some numerical examples. This chapter is the basis of the whole thesis which includes the definitions of function spaces, and the definitions and properties of boundary integral operators for the Helmholtz equation and the Maxwell's equations. The work of BEMs for the Maxwell's equations is based on the work for the Helmholtz equation and the work for the Helmholtz equation is based on the work for the Laplace equation. The work for the Laplace equation is based on some results of the study of the Laplace equation as a PDE.

\subsection{Classical electrodynamics}

In this section we introduce the Maxwell's equations for different problems in classical electrodynamics and classify them into the Poisson, heat and wave equations. We only consider electromagnetic fields in a linear, homogeneous and isotropic medium. The study of classical electrodynamics could follow [78, 30, 37].

\section{The Maxwell's equations}

In 1864 J. C. Maxwell published the famous paper to combine the equations from electrostatics and magnetostatics with Faraday law and modify them to be a consistent equation system. We call this equation system the Maxwell's equations. The Maxwell's equations are used to describe electromagnetic phenomena. In $1886 \mathrm{H}$. Hertz generated and detected electromagnetic radiation in the University of Karlsruhe.

$$
\begin{aligned}
& \nabla \cdot \mathbf{E}=\frac{\rho}{\varepsilon}, \\
& \nabla \times \mathbf{E}=-\mu \frac{\partial \mathbf{H}}{\partial t}, \\
& \nabla \cdot \mathbf{H}=0, \\
& \nabla \times \mathbf{H}=\mathbf{j}+\varepsilon \frac{\partial \mathbf{E}}{\partial t},
\end{aligned}
$$

where $\mathbf{E}$ is the electric field intensity, $\mathbf{H}$ is the magnetic field intensity, $\varepsilon$ is the permittivity, $\mu$ is the permeability, $\rho$ is the electric charge density and $\mathbf{j}$ is the electric current density. The boundary conditions at the interface between two different mediums are given by

$$
\begin{aligned}
& \mathbf{n} \cdot\left(\varepsilon_{2} \mathbf{E}_{2}-\varepsilon_{1} \mathbf{E}_{1}\right)=\Sigma \\
& \mathbf{n} \times\left(\mathbf{E}_{2}-\mathbf{E}_{1}\right)=0 \\
& \mathbf{n} \cdot\left(\mu_{2} \mathbf{H}_{2}-\mu_{1} \mathbf{H}_{1}\right)=0 \\
& \mathbf{n} \times\left(\mathbf{H}_{2}-\mathbf{H}_{1}\right)=\mathbf{K}
\end{aligned}
$$

where $\mathbf{n}$ is the unit normal on the interface, $\mu_{1}, \mu_{2}$ and $\varepsilon_{1}, \varepsilon_{2}$ are the permeability and permittivity of two different mediums, respectively, $\Sigma$ is the surface charge density, and 
$\mathbf{K}$ is the surface current density.

\section{Electrostatics (the Poisson equation)}

Electrostatics is the study of static electric fields generated by stationary electric charges. The Coulomb's law is the basis of electrostatics and the Maxwell's equations (1.1.1) are reduced to

$$
\begin{aligned}
& \nabla \cdot \mathbf{E}=\frac{\rho}{\varepsilon}, \\
& \nabla \times \mathbf{E}=0 .
\end{aligned}
$$

Since (1.1.3b) holds, we define $\mathbf{E}$ as the gradient of a scalar potential $\Phi$

$$
\mathbf{E}=-\nabla \Phi
$$

We use (1.1.4) in (1.1.3a) and get

$$
-\Delta \Phi=\frac{\rho}{\varepsilon}
$$

\section{Magnetostatics (the Poisson equation)}

Magnetostatics is the study of static magnetic fields generated by steady currents. The Biot and Savart law is the basis of magnetostatics and the Maxwell's equations (1.1.1) are reduced to

$$
\begin{aligned}
& \nabla \cdot \mathbf{H}=0, \\
& \nabla \times \mathbf{H}=\mathbf{j},
\end{aligned}
$$

Since (1.1.6a) holds, we define $\mathbf{H}$ as the curl of a vector potential $\mathbf{A}$ which satisfies the transverse gauge

$$
\begin{aligned}
& \nabla \cdot \mathbf{A}=0, \\
& \mathbf{H}=\nabla \times \mathbf{A} .
\end{aligned}
$$

We use (1.1.7b) in (1.1.6b) with (1.1.7a) and get a system of equations

$$
\begin{aligned}
\nabla \cdot \mathbf{A} & =0, \\
-\nabla^{2} \mathbf{A} & =\mathbf{j} .
\end{aligned}
$$

\section{Electromagnetics (the wave and heat equation)}

First, we consider electromagnetic fields in a dielectric medium. Since (1.1.1c) holds, we have the same definition for $\mathbf{H}$ as (1.1.7b). We use (1.1.7b) in (1.1.1b) and get

$$
\nabla \times\left(\mathbf{E}+\mu \frac{\partial \mathbf{A}}{\partial t}\right)=0 .
$$


According (1.1.9) we define $\mathbf{E}+\mu \frac{\partial \mathbf{A}}{\partial t}$ as the gradient of a scalar potential

$$
\mathbf{E}+\mu \frac{\partial \mathbf{A}}{\partial t}=-\nabla \Phi
$$

We could use the Lorenz gauge or the transverse gauge for $\Phi$ and A. By the Lorenz gauge (1.1.10a) we get a system of equations

$$
\begin{aligned}
& \nabla \cdot \mathbf{A}+\varepsilon \frac{\partial \Phi}{\partial t}=0 \\
& \varepsilon \mu \frac{\partial^{2} \Phi}{\partial t^{2}}-\Delta \Phi=\frac{\rho}{\varepsilon} \\
& \varepsilon \mu \frac{\partial^{2} \mathbf{A}}{\partial t^{2}}-\nabla^{2} \mathbf{A}=\mathbf{j} .
\end{aligned}
$$

For the electric field intensity we use (1.1.1d) in (1.1.1b) and get

$$
\varepsilon \mu \frac{\partial^{2} \mathbf{E}}{\partial t^{2}}-\nabla^{2} \mathbf{E}=-\mu \frac{\partial \mathbf{j}}{\partial t}
$$

We could also use (1.1.1b) in (1.1.1d) to get an equation for $\mathbf{H}$ which is similar to (1.1.11).

Next, we consider electromagnetic fields in a conducting medium, so (1.1.1d) has a different form by the Ohm's law

$$
\nabla \times \mathbf{H}=\mathbf{j}+\sigma \mathbf{E}+\varepsilon \frac{\partial \mathbf{E}}{\partial t},
$$

where $\sigma$ is the electrical conductivity. Using the same definitions of the scalar and vector potentials and the Lorenz gauge (1.1.13a), we get a system of equations

$$
\begin{aligned}
& \nabla \cdot \mathbf{A}+\sigma \Phi+\varepsilon \frac{\partial \Phi}{\partial t}=0 \\
& \varepsilon \mu \frac{\partial^{2} \Phi}{\partial t^{2}}+\mu \sigma \frac{\partial \Phi}{\partial t}-\Delta \Phi=\frac{\rho}{\varepsilon} \\
& \varepsilon \mu \frac{\partial^{2} \mathbf{A}}{\partial t^{2}}+\mu \sigma \frac{\partial \mathbf{A}}{\partial t}-\nabla^{2} \mathbf{A}=\mathbf{j} .
\end{aligned}
$$

For the electric field intensity we use (1.1.12) in (1.1.1b) and get

$$
\varepsilon \mu \frac{\partial^{2} \mathbf{E}}{\partial t^{2}}+\mu \sigma \frac{\partial \mathbf{E}}{\partial t}-\nabla^{2} \mathbf{E}=-\mu \frac{\partial \mathbf{j}}{\partial t} .
$$

Then, for a conducting medium, $\sigma$ is much larger than $\varepsilon$, so we may neglect the second order differential terms in (1.1.13b), (1.1.13c) and (1.1.14) and get

$$
\begin{gathered}
\mu \sigma \frac{\partial \Phi}{\partial t}-\Delta \Phi=\frac{\rho}{\varepsilon} \\
\mu \sigma \frac{\partial \mathbf{A}}{\partial t}-\nabla^{2} \mathbf{A}=\mathbf{j}
\end{gathered}
$$




$$
\mu \sigma \frac{\partial \mathbf{E}}{\partial t}-\nabla^{2} \mathbf{E}=-\mu \frac{\partial \mathbf{j}}{\partial t}
$$

\section{Electromagnetic waves (the Helmholtz equation)}

The study of the propagation of electromagnetic waves is a common topic in classical electrodynamics. We just consider the equations for the electric field intensity E. First, we consider electromagnetic waves in a dielectric medium without sources $(\mathbf{j}$ and $\rho$ ). From (1.1.11) we get

$$
\varepsilon \mu \frac{\partial^{2} \mathbf{E}}{\partial t^{2}}-\nabla^{2} \mathbf{E}=0
$$

Next, we consider electromagnetic waves in a conducting medium without sources. From (1.1.14) we get

$$
\varepsilon \mu \frac{\partial^{2} \mathbf{E}}{\partial t^{2}}+\mu \sigma \frac{\partial \mathbf{E}}{\partial t}-\nabla^{2} \mathbf{E}=0 .
$$

Then, we neglect the second order differential term in (1.1.18) and get

$$
\mu \sigma \frac{\partial \mathbf{E}}{\partial t}-\nabla^{2} \mathbf{E}=0
$$

Let $\omega$ be a certain frequency of electromagnetic waves. We use $\mathbf{E}(x, t)=\mathbf{e}(x) \mathrm{e}^{-\mathrm{i} \omega t}$ in (1.1.17), (1.1.18), (1.1.19) and (1.1.1a). We get a system of equations for time-harmonic electric fields

$$
\begin{aligned}
\nabla \cdot \mathbf{e} & =0 \\
-\nabla^{2} \mathbf{e} & =\lambda \mathbf{e}
\end{aligned}
$$

where $\lambda=\varepsilon \mu \omega^{2}$ for (1.1.17), $\lambda=\varepsilon \mu \omega^{2}+\mathrm{i} \mu \omega \sigma$ for (1.1.18), or $\lambda=\mathrm{i} \mu \omega \sigma$ for (1.1.19). We can do the same transformations for the corresponding equations for $\mathbf{H}$.

\section{Classification}

Now we have already seen some familiar equations in classical electrodynamics. $\Phi$ in (1.1.5) and $\mathbf{A}_{i}$ in (1.1.8) satisfy the Poisson equation respectively. $\Phi, \mathbf{A}_{i}$ and $\mathbf{E}_{i}$ satisfy the wave equation in (1.1.10) and (1.1.11) respectively. They also satisfy the wave equation with a damping term in (1.1.13) and (1.1.14) and the heat equation in (1.1.15) and (1.1.16) respectively. $\mathbf{e}_{i}$ satisfies the Helmholtz equation and $\lambda$ is a real number or a complex number with zero or nonzero real part in (1.1.20). The above equations with appropriate boundary and initial conditions will be boundary value problems or initial-boundary value problems. If we calculate $\lambda$ and e together in (1.1.20), this is an eigenvalue problem.

\subsection{The Helmholtz case}

From (1.1.20) we know that $\mathbf{e}_{i}$ satisfies the Helmholtz equation and $\lambda$ could be a real number or a complex number. In this section we study Dirichlet and Neumann boundary value problems for the Helmholtz equation. We just consider electromagnetic waves in a 
dielectric medium, so $\lambda$ is a real number. We assume that $\lambda=k^{2}$ and $k$ is a positive real number. The Helmholtz equation is

$$
\Delta u(x)+k^{2} u(x)=0 \quad \forall x \in \Omega,
$$

where $\Omega$ is a bounded domain. Dirichlet and Neumann boundary conditions are given by

$$
\begin{array}{ll}
u(x)=f(x) & \forall x \in \Gamma, \\
\frac{\partial u}{\partial \mathbf{n}}(x):=\mathbf{n}(x) \cdot \nabla u(x)=g(x) & \forall x \in \Gamma,
\end{array}
$$

respectively, where $\Gamma:=\partial \Omega, \mathbf{n}$ is the exterior unit normal to $\Gamma$, and $f$ and $g$ are the given data.

\subsubsection{Representation formula}

We assume that $\Gamma$ is a smooth boundary. Green's first formula for (1.2.1) is

$$
\begin{array}{r}
\int_{\Omega}\left(\Delta u(x)+k^{2} u(x)\right) v(x) \mathrm{d} x+\int_{\Omega}\left(\nabla u(x) \cdot \nabla v(x)-k^{2} u(x) v(x)\right) \mathrm{d} x \\
=\int_{\Gamma} \frac{\partial u}{\partial \mathbf{n}}(x) v(x) \mathrm{d} s_{x} .
\end{array}
$$

for any smooth function $v$. Green's second formula is

$$
\begin{aligned}
\int_{\Omega}\left(\Delta u(x)+k^{2} u(x)\right) v(x) \mathrm{d} x- & \int_{\Omega} u(x)\left(\Delta v(x)+k^{2} v(x)\right) \mathrm{d} x \\
& =\int_{\Gamma} \frac{\partial u}{\partial \mathbf{n}}(x) v(x) \mathrm{d} s_{x}-\int_{\Gamma} u(x) \frac{\partial v}{\partial \mathbf{n}}(x) \mathrm{d} s_{x} .
\end{aligned}
$$

The fundamental solution for the Helmholtz equation is

$$
E_{k}(x, y)=\frac{\mathrm{e}^{\mathrm{i} k|x-y|}}{4 \pi|x-y|}
$$

which satisfies

$$
-\left(\Delta+k^{2}\right) E_{k}(x, y)=\delta(x-y) .
$$

We use $v(x)=E_{k}(x, y)$ and (1.2.1) in (1.2.4), and exchange the notations for $x$ and $y$. Then we get a representation formula for $u$,

$$
u(x)=\int_{\Gamma} E_{k}(x, y) \frac{\partial u}{\partial \mathbf{n}_{y}}(y) \mathrm{d} s_{y}-\int_{\Gamma} \frac{\partial E_{k}(x, y)}{\partial \mathbf{n}_{y}} u(y) \mathrm{d} s_{y} \quad \forall x \in \Omega .
$$

\subsubsection{Function spaces}

In this section we introduce some basic notation and list the definitions of the function spaces which we will use. Let $\alpha=\left(\alpha_{1}, \cdots, \alpha_{d}\right)$ be a $d$-dimensional vector and $\alpha_{i}$ be 
non-negative integers. $|\alpha|:=\sum_{i=1}^{d} \alpha_{i}$. Then we define partial derivatives for a real valued function $u$ which is sufficiently smooth as

$$
D^{\alpha} u:=\frac{\partial^{|\alpha|} u}{\partial x_{1}^{\alpha_{1}} \cdots \partial x_{d}^{\alpha_{d}}} .
$$

Let $\Omega$ be a bounded domain. In our work a domain means an open and connected subset of $\mathbb{R}^{n}(n=2,3)$. The definitions of continuously differentiable function spaces $C^{m}(\Omega)$ and Hölder continuously differentiable function spaces $C^{m+\beta}(\Omega)$ for $m \in \mathbb{N}^{0}$ and $0<\beta<1$ are

$$
C^{m}(\Omega):=\{u \mid u \text { is } m \text { times continuously differentiable in } \Omega\},
$$

and

$$
C^{m+\beta}(\Omega):=\left\{u \in C^{m}(\Omega) \mid\|u\|_{C^{m+\beta}(\Omega)}<\infty\right\},
$$

with the corresponding norms

$$
\|u\|_{C^{m}(\Omega)}:=\sum_{|\alpha| \leq m} \sup _{x \in \Omega}\left|D^{\alpha} u(x)\right|
$$

and

$$
\|u\|_{C^{m+\beta}(\Omega)}:=\|u\|_{C^{m}(\Omega)}+\sum_{|\alpha|=m} \sup _{x, y \in \Omega, x \neq y} \frac{\left|D^{\alpha} u(x)-D^{\alpha} u(y)\right|}{|x-y|^{\beta}} .
$$

$C^{\infty}(\Omega)$ is the space of functions which are infinitely continuously differentiable.

$L^{2}(\Omega)$ is a Hilbert space and the definition is

$$
L^{2}(\Omega):=\left\{u \mid u \text { is a measurable function },\|u\|_{L^{2}(\Omega)}<\infty\right\},
$$

where

$$
\|u\|_{L^{2}(\Omega)}^{2}:=\int_{\Omega}|u(x)|^{2} \mathrm{~d} x .
$$

The definition of the inner product of $L^{2}(\Omega)$ is

$$
\langle u, v\rangle_{L^{2}(\Omega)}:=\int_{\Omega} u(x) \overline{v(x)} \mathrm{d} x .
$$

We can define Sobolev spaces as the closure of smooth function spaces by the norm defined by weak derivatives. Alternatively, we can define Sobolev spaces as a subset of distributions. We use the first definition with the notation from the second definition.

$$
H^{m}(\Omega):={\overline{C^{\infty}(\Omega)}}^{\|\cdot\|_{H^{m}(\Omega)}},
$$

with the norm

$$
\begin{aligned}
\|u\|_{H^{m}(\Omega)}^{2} & :=\sum_{\alpha \leq m}\left\|D^{\alpha} u\right\|_{L^{2}(\Omega)}^{2} . \\
H^{m+\beta}(\Omega) & :=\overline{C^{\infty}(\Omega)}\|\cdot\|_{H^{m+\beta}(\Omega)},
\end{aligned}
$$


with the norm

$$
\|u\|_{H^{m+\beta}(\Omega)}^{2}:=\|u\|_{H^{m}(\Omega)}^{2}+|u|_{H^{m+\beta}(\Omega)}^{2},
$$

and the semi-norm is

$$
|u|_{H^{m+\beta}(\Omega)}^{2}:=\sum_{|\alpha|=m} \int_{\Omega} \int_{\Omega} \frac{\left|D^{\alpha} u(x)-D^{\alpha} u(y)\right|^{2}}{|x-y|^{n+2 \beta}} \mathrm{d} x \mathrm{~d} y .
$$

$n$ is the dimension of the Euclidean space. $\beta \in(0,1) . H^{-(m+\beta)}(\Omega)$ is the dual space of $H^{m+\beta}(\Omega)$.

For boundary integral equations we need Sobolev spaces defined on the manifold $\Gamma$. We can define Sobolev spaces on $\Gamma$ by the same way as on $\Omega$, or by the trace inequality and the inverse trace inequality from [65, Theorem 2.21 and 2.22] and [1, 48], we can also define Sobolev spaces on $\Gamma$ as the trace spaces of Sobolev spaces on $\Omega$. That is

$$
H^{m+\beta}(\Gamma):=\left\{\left.u\right|_{\Gamma} \mid u \in H^{m+\beta+1 / 2}(\Omega)\right\} .
$$

$H^{-(m+\beta)}(\Gamma)$ is the dual space of $H^{m+\beta}(\Gamma)$.

\subsubsection{Boundary integral equations}

When we derive the representation formula (1.2.6), we assume that the boundary is smooth and $u \in C^{2}(\Omega)$. We call (1.2.6) the classical representation formula. In the last section we have introduced (2a) in Fig. 1.1 and in this section we continue to introduce (2b) and (2c), then we can generalize the problem for (1.2.6).

Let $u$ be the solution of (1.2.1). From Dirichlet and Neumann boundary conditions (1.2.2a) and (1.2.2b), we need to define two trace operators.

$$
\gamma_{0}(u)(x):=\lim _{\tilde{x} \in \Omega \rightarrow x \in \Gamma} u(\tilde{x})
$$

We define $\gamma_{1}(u)$ as the solution of the variational formulation

$$
\left\langle\gamma_{1}(u), v\right\rangle_{\Gamma}:=\int_{\Omega}\left(\nabla u(x) \cdot \nabla \mathcal{E}(v)(x)-k^{2} u(x) \mathcal{E}(v)(x)\right) \mathrm{d} x
$$

for all $v \in H^{1 / 2}(\Gamma) .\langle\cdot, \cdot\rangle_{\Gamma}$ is the dual pairing. The operator $\mathcal{E}: H^{1 / 2}(\Gamma) \rightarrow H^{1}(\Omega)$ is defined by the inverse trace inequality [65, Theorem 2.22]. Then for $u \in H^{1}(\Omega)$ we have $\gamma_{0}(u) \in H^{1 / 2}(\Gamma)$ by the trace inequality and $\gamma_{1}(u) \in H^{-1 / 2}(\Gamma)$. We call $\gamma_{0}$ the Dirichlet trace operator and $\gamma_{1}$ the Neumann trace operator. We have a lemma for the continuity of the trace operators.

Lemma 1. Let $\Gamma$ be a Lipschitz boundary. The Dirichlet trace operator $\gamma_{0}$ and the Neumann trace operator $\gamma_{1}$ for the Helmholtz equation are bounded operators

$$
\begin{aligned}
& \gamma_{0}: H^{1}(\Omega) \rightarrow H^{1 / 2}(\Gamma), \\
& \gamma_{1}: H^{1}(\Omega) \rightarrow H^{-1 / 2}(\Gamma) .
\end{aligned}
$$


Proof. See [1, 48] and Lemma 4.4 in [65].

We define two potential operators corresponding to the two boundary integrals in (1.2.6) for $v \in H^{-1 / 2}(\Gamma)$ and $w \in H^{1 / 2}(\Gamma)$ as

$$
\begin{array}{cl}
\widetilde{V}_{k}(v)(x)=\int_{\Gamma} E_{k}(x, y) v(y) \mathrm{d} s_{y} & \forall x \in \Omega, \\
W_{k}(w)(x)=\int_{\Gamma} \frac{\partial E_{k}(x, y)}{\partial \mathbf{n}(y)} w(y) \mathrm{d} s_{y} & \forall x \in \Omega .
\end{array}
$$

We call $\widetilde{V}_{k}$ the single-layer potential operator and $W_{k}$ the double-layer potential operator. We have a lemma for the continuity of $\widetilde{V}_{k}$ and $W_{k}$.

Lemma 2. Let $\Gamma$ be a Lipschitz boundary and let $v \in H^{-1 / 2}(\Gamma)$ or $w \in H^{1 / 2}(\Gamma)$ be given. Then, $u(x)=\widetilde{V}_{k}(v)(x)$ or $u(x)=W_{k}(w)(x)$ for $x \in \Omega$ is a weak solution of the Helmholtz equation. The single-layer potential operator $\widetilde{V}_{k}$ and the double-layer potential operator $W_{k}$ are bounded operators

$$
\begin{gathered}
\widetilde{V}_{k}: H^{-1 / 2}(\Gamma) \rightarrow H^{1}(\Omega), \\
W_{k}: H^{1 / 2}(\Gamma) \rightarrow H^{1}(\Omega) .
\end{gathered}
$$

Proof. See Lemma 6.6 and Lemma 6.10 in [65].

By using the Dirichlet and Neumann trace operators to the single-layer and doublelayer potential operators, respectively, we could define four boundary integral operators for the Helmholtz equation. By the continuity of trace operators and potential operators, we also have the continuity of boundary integral operators. Then we get the following theorem.

Theorem 1. Let $\Gamma$ be a Lipschitz boundary. The boundary integral operators, $\gamma_{0} \widetilde{V}_{k}, \gamma_{0} W_{k}$, $\gamma_{1} \widetilde{V}_{k}$, and $\gamma_{1} W_{k}$, are bounded operators

$$
\begin{aligned}
\gamma_{0} \widetilde{V}_{k}: & H^{-1 / 2}(\Gamma) \rightarrow H^{1 / 2}(\Gamma), \\
\gamma_{0} W_{k}: & H^{1 / 2}(\Gamma) \rightarrow H^{1 / 2}(\Gamma), \\
\gamma_{1} \widetilde{V}_{k}: & H^{-1 / 2}(\Gamma) \rightarrow H^{-1 / 2}(\Gamma), \\
\gamma_{1} W_{k}: & H^{1 / 2}(\Gamma) \rightarrow H^{-1 / 2}(\Gamma) .
\end{aligned}
$$

Proof. By using Lemma 1 and Lemma 2.

In the calculation we need explicit formulae for the boundary integral operators. We 
have the following definitions

$$
\begin{aligned}
\left(V_{k} v\right)(x) & :=\int_{\Gamma \backslash\{x\}} E_{k}(x, y) v(y) \mathrm{d} s_{y}, \\
\left(K_{k} w\right)(x) & :=\text { p.v. } \int_{\Gamma \backslash\{x\}} \gamma_{1, y}\left(E_{k}(x, y)\right) w(y) \mathrm{d} s_{y}, \\
\left(K_{k}^{\prime} v\right)(x) & :=\text { p.v. } \int_{\Gamma \backslash\{x\}} \gamma_{1, x}\left(E_{k}(x, y)\right) v(y) \mathrm{d} s_{y}, \\
\left(D_{k} w\right)(x) & :=- \text { p.v. } \int_{\Gamma} \gamma_{1, x}\left(\gamma_{1, y}\left(E_{k}(x, y)\right)\right)(w(y)-w(x)) \mathrm{d} s_{y},
\end{aligned}
$$

for $x \in \Gamma, v \in H^{-1 / 2}(\Gamma)$ and $w \in H^{1 / 2}(\Gamma) . \quad V_{k}$ is called the single-layer boundary integral operator and $V_{k} v$ is a weakly singular integral. $K_{k}$ and $K_{k}^{\prime}$ are called the doublelayer and adjoint double-layer boundary integral operator, respectively, and $K_{k} w$ and $K_{k}^{\prime} v$ are Cauchy principle value integrals. $D_{k}$ is called the hyper-singular boundary integral operator. For the hyper-singular boundary integral operator, it is not integrable. We need to do a regularization for it. (1.2.7d) is a regularization of $D_{k}$ and it is a Cauchy principle value integral. Then we have the following results for the boundary integral operators. There exists $\eta \in L^{\infty}(\Gamma)$ such that

$$
\begin{aligned}
V_{k}=\gamma_{0} \widetilde{V}_{k}: & H^{-1 / 2}(\Gamma) \rightarrow H^{1 / 2}(\Gamma), \\
(-1+\eta) I+K_{k}=\gamma_{0} W_{k}: & H^{1 / 2}(\Gamma) \rightarrow H^{1 / 2}(\Gamma) \\
\eta I+K_{k}^{\prime}=\gamma_{1} \widetilde{V}_{k}: & H^{-1 / 2}(\Gamma) \rightarrow H^{-1 / 2}(\Gamma), \\
D_{k}=\gamma_{1} W_{k}: & H^{1 / 2}(\Gamma) \rightarrow H^{-1 / 2}(\Gamma) .
\end{aligned}
$$

More details see [65, 49].

If $\Gamma$ is differentiable within a neighborhood of $x \in \Gamma$, we have $\eta(x)=\frac{1}{2}$. So without loss of generality, we always assume that $\eta(x)=\frac{1}{2}$ for almost all $x \in \Gamma$ in our work. (1.2.7d) is still not enough for the calculation, we have the other formula for the dual pairing $\left\langle D_{k} w, v\right\rangle_{\Gamma}$,

$$
\begin{aligned}
\left\langle D_{k} w, v\right\rangle_{\Gamma}= & \int_{\Gamma} \int_{\Gamma} E_{k}(x, y)(\mathbf{n}(y) \times \nabla \widetilde{w}(y)) \cdot(\mathbf{n}(x) \times \nabla \widetilde{v}(x)) \mathrm{d} s_{y} \mathrm{~d} s_{x} \\
& -k^{2} \int_{\Gamma} \int_{\Gamma} E_{k}(x, y) w(y) v(x) \mathbf{n}(y) \cdot \mathbf{n}(x) \mathrm{d} s_{y} \mathrm{~d} s_{x}
\end{aligned}
$$

where $\widetilde{w}$ and $\widetilde{v}$ are the suitable extensions of $w$ and $v$ into a three-dimensional neighborhood of $\Gamma$ respectively. For more details see $[65,51]$.

Now we go back to the representation formula (1.2.6). The function space for $u$ is extended to $H^{1}(\Omega)$ and we have

$$
u(x)=\left(\widetilde{V}_{k} \gamma_{1}(u)\right)(x)-\left(W_{k} \gamma_{0}(u)\right)(x) \quad \forall x \in \Omega .
$$

We call (1.2.9) the generalized representation formula. We use the Dirichlet trace operator 
$\gamma_{0}$ and the Neumann trace operator $\gamma_{1}$ on (1.2.9), respectively, and get two boundary integral equations

$$
\begin{aligned}
& \gamma_{0} u=V_{k}\left(\gamma_{1} u\right)-\left(-\frac{1}{2} I+K_{k}\right)\left(\gamma_{0} u\right), \\
& \gamma_{1} u=\left(\frac{1}{2} I+K_{k}^{\prime}\right)\left(\gamma_{1} u\right)+D_{k}\left(\gamma_{0} u\right),
\end{aligned}
$$

for $\gamma_{0} u \in H^{1 / 2}(\Gamma)$ and $\gamma_{1} u \in H^{-1 / 2}(\Gamma)$. If we want to use the generalized representation formula (1.2.9) to calculate the solution in the domain, we need to know $\left(\gamma_{0} u, \gamma_{1} u\right)$ on the whole boundary. So by using (1.2.10), we define a Calderón projection for $\left(\gamma_{0} u, \gamma_{1} u\right)$ as

$$
\mathcal{C}=\left(\begin{array}{cc}
\frac{1}{2} I-K_{k} & V_{k} \\
D_{k} & \frac{1}{2} I+K_{k}^{\prime}
\end{array}\right): H^{1 / 2}(\Gamma) \times H^{-1 / 2}(\Gamma) \rightarrow H^{1 / 2}(\Gamma) \times H^{-1 / 2}(\Gamma) .
$$

By using the Dirichlet boundary condition (1.2.2a) and the Neumann boundary condition (1.2.2b), respectively, we get two boundary integral equations

$$
\begin{aligned}
& V_{k}\left(\gamma_{1} u\right)=\left(\frac{1}{2} I+K_{k}\right) f, \\
& D_{k}\left(\gamma_{0} u\right)=\left(\frac{1}{2} I-K_{k}^{\prime}\right) g .
\end{aligned}
$$

\subsubsection{Variational formulations}

In the last section we consider the step (1a) in Fig. 1.1. In this section we continue to introduce the step (1b) in Fig. 1.1. We define $\sigma:=\gamma_{1} u$ and $\varphi:=\gamma_{0} u$.

The variational formulation for (1.2.12) is to find $\sigma \in H^{-1 / 2}(\Gamma)$ such that

$$
\left\langle V_{k} \sigma, \chi\right\rangle_{\Gamma}=\left\langle\left(\frac{1}{2} I+K_{k}\right) f, \chi\right\rangle_{\Gamma}
$$

for all $\chi \in H^{-1 / 2}(\Gamma)$.

The variational formulation for (1.2.13) is to find $\varphi \in H^{1 / 2}(\Gamma)$ such that

$$
\left\langle D_{k} \varphi, v\right\rangle_{\Gamma}=\left\langle\left(\frac{1}{2} I-K_{k}^{\prime}\right) g, v\right\rangle_{\Gamma}
$$

for all $v \in H^{1 / 2}(\Gamma)$.

If $k=0,(1.2 .1)$ is the Laplace equation. Let $V$ denote the single-layer boundary integral operator and $D$ denote the hyper-singular boundary integral operator of the Laplace equation. $V$ is proved to be $H^{-1 / 2}(\Gamma)$-elliptic and $D$ is proved to be $H^{1 / 2}(\Gamma)$ semi-elliptic. $V_{k}$ is not $H^{-1 / 2}(\Gamma)$-elliptic and $D_{k}$ is not $H^{1 / 2}(\Gamma)$-elliptic for the Helmholtz equation. If we want to prove the existence and uniqueness of the solutions of (1.2.14) and (1.2.15), we need to prove the coercivity of $V_{k}$ and $D_{k}$. The coercivity means that for a bounded operator there exists a compact operator such that the addition of these two 
operators is an elliptic operator. We have a lemma for the coercivity of $V_{k}$ and $D_{k}$.

Lemma 3. Let $\Gamma$ be a Lipschitz boundary. Let $C_{V}:=V-V_{k}$ and $C_{D}:=D+I-D_{k}$. Then $C_{V}$ and $C_{D}$ are compact operators, and $V_{k}$ and $D_{k}$ satisfy the Gårding inequality

$$
\begin{gathered}
\left\langle\left(V_{k}+C_{V}\right) v, v\right\rangle_{\Gamma}=\langle V v, v\rangle_{\Gamma} \geq c^{V}\|v\|_{H^{-1 / 2}(\Gamma)}^{2}, \\
\left\langle\left(D_{k}+C_{D}\right) w, w\right\rangle_{\Gamma}=\langle(D+I) w, w\rangle_{\Gamma} \geq c^{D}\|w\|_{H^{1 / 2}(\Gamma)}^{2}
\end{gathered}
$$

for $v \in H^{-1 / 2}(\Gamma)$ and $w \in H^{1 / 2}(\Gamma)$.

Proof. More details see Theorem 6.40 in [65].

If $k^{2}$ is not an eigenvalue of the Laplace eigenvalue problem, by the Fredholm's alternative and Lemma 3, we have the existence and uniqueness of (1.2.14) and (1.2.15).

\subsubsection{Galerkin-BEMs}

In this section we go to the last step (1c) in Fig. 1.1 to introduce Galerkin-BEMs for (1.2.14) and (1.2.15). In our work we only consider triangular meshes.

Definition 1. A triangular cell $\mathcal{C}$ consists of a domain $\Omega_{\mathcal{C}}$, vertices $\mathcal{V}=\left\{x_{1}, x_{2}, x_{3}\right\}$ and edges $\mathcal{E}=\left\{e_{1}, e_{2}, e_{3}\right\}$.

Definition 2. A triangular mesh $\Gamma_{h}$ is built by cells $\left\{\mathcal{C}_{i}\right\}_{i=1}^{N}$ and defines a boundary $\Gamma_{h}$ such that $\Gamma_{h}=\bigcup_{i=1}^{N} \bar{\Omega}_{i}, \mathcal{V}_{\Gamma_{h}}=\bigcup_{i=1}^{N} \mathcal{V}_{i}$, and $\mathcal{E}_{\Gamma_{h}}=\bigcup_{i=1}^{N} \mathcal{E}_{i}$.

Def. 1 and 2 are important for the data structure of meshes in $\mathrm{M}++$ which is a parallel FEM software developed by Prof. C. Wieners in KIT. More details see [74, 76, 75].

Definition 3. A boundary element is defined by $\left(\mathcal{C}, P_{\mathcal{C}}, \Sigma_{\mathcal{C}}\right) . P_{\mathcal{C}}$ is a polynomial function space on $\Omega_{\mathcal{C}} . \Sigma_{\mathcal{C}}$ is a set of linear functionals on $P_{\mathcal{C}}$ and they are called the degrees of freedom.

A cell $\mathcal{C}$ and a boundary element $\left(\mathcal{C}, P_{\mathcal{C}}, \Sigma_{\mathcal{C}}\right)$ could also be defined from a reference cell $\widehat{\mathcal{C}}$ and a reference boundary element $\left(\widehat{\mathcal{C}}, P_{\widehat{\mathcal{C}}}, \Sigma_{\widehat{\mathcal{C}}}\right)$ by a mapping.

We define two piecewise polynomial function spaces on the boundary

$$
\begin{gathered}
S_{h}^{0}\left(\Gamma_{h}\right):=\left\{v_{h} \in L^{2}\left(\Gamma_{h}\right) \mid v_{h} \text { is constant on every } \Omega_{i} \text { in the mesh } \Gamma_{h}\right\}, \\
S_{h}^{1}\left(\Gamma_{h}\right):=\left\{w_{h} \in C\left(\Gamma_{h}\right) \mid w_{h} \text { is a linear function on every } \Omega_{i} \text { in the mesh } \Gamma_{h}\right\} .
\end{gathered}
$$

Boundary elements on every $\mathcal{C}_{i}$ in the mesh $\Gamma_{h}$ with polynomial function spaces defined by $\left.S_{h}^{0}\left(\Gamma_{h}\right)\right|_{\Omega_{i}}$ and $\left.S_{h}^{1}\left(\Gamma_{h}\right)\right|_{\Omega_{i}}$ are $\left(\mathcal{C}_{i},\left.S_{h}^{0}\left(\Gamma_{h}\right)\right|_{\Omega_{i}}, \Sigma_{\mathcal{C}_{i}}^{0}\right)$ and $\left(\mathcal{C}_{i},\left.S_{h}^{1}\left(\Gamma_{h}\right)\right|_{\Omega_{i}}, \Sigma_{\mathcal{C}_{i}}^{1}\right)$, and

$$
\begin{gathered}
\Sigma_{\mathcal{C}_{i}}^{0}:=\left\{l \text { is a lineal functional, } l(v)=v\left(\left(x_{1}+x_{2}+x_{3}\right) / 3\right),\left.v \in S_{h}^{0}\left(\Gamma_{h}\right)\right|_{\Omega_{i}}\right\}, \\
\Sigma_{\mathcal{C}_{i}}^{1}:=\left\{l_{i} \text { is a linear functional for } i=1,2,3, l_{i}(w)=w\left(x_{i}\right),\left.w \in S_{h}^{1}\left(\Gamma_{h}\right)\right|_{\Omega_{i}}\right\} .
\end{gathered}
$$

Then we define two boundary element spaces for (1.2.14) and (1.2.15) by $\left(\Gamma_{h}, S_{h}^{0}\left(\Gamma_{h}\right), \Sigma_{\Gamma_{h}}^{0}\right)$ and $\left(\Gamma_{h}, S_{h}^{1}\left(\Gamma_{h}\right), \Sigma_{\Gamma_{h}}^{1}\right) . \Sigma_{\Gamma_{h}}^{0}$ and $\Sigma_{\Gamma_{h}}^{1}$ are the union of the degrees of freedom of all $\Sigma_{\mathcal{C}_{i}}^{0}$ and 
$\Sigma_{\mathcal{C}_{i}}^{1}$. We simplify the notations from $\left(\Gamma_{h}, S_{h}^{0}\left(\Gamma_{h}\right), \Sigma_{\Gamma_{h}}^{0}\right)$ and $\left(\Gamma_{h}, S_{h}^{1}\left(\Gamma_{h}\right), \Sigma_{\Gamma_{h}}^{1}\right)$ to $S_{h}^{0}\left(\Gamma_{h}\right)$ and $S_{h}^{1}\left(\Gamma_{h}\right)$.

The discretization of (1.2.14) is to find $\sigma_{h} \in S_{h}^{0}\left(\Gamma_{h}\right)$ such that

$$
\left\langle V_{k} \sigma_{h}, \chi_{h}\right\rangle_{\Gamma}=\left\langle\left(\frac{1}{2} I+K_{k}\right) f, \chi_{h}\right\rangle_{\Gamma}
$$

for all $\chi_{h} \in S_{h}^{0}\left(\Gamma_{h}\right)$.

The discretization of (1.2.15) is to find $\varphi_{h} \in S_{h}^{1}\left(\Gamma_{h}\right)$ such that

$$
\left\langle D_{k} \varphi_{h}, v_{h}\right\rangle_{\Gamma}=\left\langle\left(\frac{1}{2} I-K_{k}^{\prime}\right) g, v_{h}\right\rangle_{\Gamma},
$$

for all $v_{h} \in S_{h}^{1}\left(\Gamma_{h}\right)$.

Let $\left\{\Phi_{i}^{0}\right\}_{i=1}^{N_{0}}$ be a basis of $S_{h}^{0}\left(\Gamma_{h}\right)$ and $\left\{\Phi_{i}^{1}\right\}_{i=1}^{N_{1}}$ be a basis of $S_{h}^{1}\left(\Gamma_{h}\right)$. We use $\sigma_{h}=$ $\sum_{j=1}^{N_{0}} \xi_{j}^{0} \Phi_{j}^{0}$ and $\varphi_{h}=\sum_{j=1}^{N_{1}} \xi_{j}^{1} \Phi_{j}^{1}$ in (1.2.18) and (1.2.19) and get two linear systems.

$$
\begin{gathered}
A^{0} \xi^{0}=b^{0} \\
A^{0}[i, j]=\frac{1}{4 \pi} \int_{\Omega_{i}} \int_{\Omega_{j}} \frac{\mathrm{e}^{\mathrm{i} k|x-y|}}{|x-y|} \mathrm{d} s_{y} \mathrm{~d} s_{x} \\
b^{0}[i]=\frac{1}{2} \int_{\Omega_{i}} f(x) \mathrm{d} s_{x}+\frac{1}{4 \pi} \int_{\Omega_{i}} \int_{\Gamma}(1-\mathrm{i} k|x-y|) \mathrm{e}^{\mathrm{i} k|x-y|} \frac{(x-y) \cdot \mathbf{n}(y)}{|x-y|^{3}} f(y) \mathrm{d} s_{y} \mathrm{~d} s_{x} \\
A^{1}[i, j]=\frac{1}{4 \pi} \int_{\Gamma} \int_{\Gamma} \frac{\mathrm{e}^{\mathrm{i} k|x-y|}}{|x-y|}\left(\mathbf{n}(y) \times \nabla \widetilde{\Phi_{j}^{1}}(y)\right) \cdot\left(\mathbf{n}(x) \times \nabla \widetilde{\Phi_{i}^{1}}(x)\right) \mathrm{d} s_{y} \mathrm{~d} s_{x} \\
b_{\Gamma}^{1} \int_{\Gamma} \frac{\mathrm{e}^{\mathrm{i} k|x-y|}}{|x-y|} \Phi_{j}^{1}(y) \Phi_{i}^{1}(x) \mathbf{n}(y) \cdot \mathbf{n}(x) \mathrm{d} s_{y} \mathrm{~d} s_{x} \\
\int_{\Gamma} g(x) \Phi_{i}^{1}(x) \mathrm{d} s_{x}-\frac{1}{4 \pi} \int_{\Gamma} \int_{\Gamma}(1-\mathrm{i} k|x-y|) \mathrm{e}^{\mathrm{i} k|x-y|} \frac{(y-x) \cdot \mathbf{n}(x)}{|x-y|^{3}} g(y) \Phi_{i}^{1}(x) \mathrm{d} s_{y} \mathrm{~d} s_{x}
\end{gathered}
$$

The last step is the a Priori error estimates for (1.2.18) and (1.2.19). The first error estimate is the Cea's lemma which constructs the error by the best approximation. The Cea's lemma is based on the stability from the well-known Ladyzenskaya-Babuška-Brezzi condition (LBB condition). The LBB condition is from the Garding inequality and the uniqueness of $V_{k}, D_{k}$. We prove the LBB condition firstly following [34, Theorem 5.5].

Lemma 4. There exists $h_{0}>0$ such that for all $\chi_{h} \in S_{h}^{0}\left(\Gamma_{h}\right)$ and $v_{h} \in S_{h}^{1}\left(\Gamma_{h}\right)$ and $0<h<h_{0}$ we have

$$
\begin{aligned}
c_{s}\left\|\chi_{h}\right\|_{H^{-1 / 2}(\Gamma)} & \leq \sup _{\chi_{h}^{\prime} \in S_{h}^{0}\left(\Gamma_{h}\right),\left\|\chi_{h}^{\prime}\right\|_{H^{-1 / 2}(\Gamma)}>0} \frac{\left|\left\langle V_{k} \chi_{h}, \chi_{h}^{\prime}\right\rangle_{\Gamma}\right|}{\left\|\chi_{h}^{\prime}\right\|_{H^{-1 / 2}(\Gamma)}}, \\
c_{d}\left\|v_{h}\right\|_{H^{1 / 2}(\Gamma)} & \leq \sup _{v_{h}^{\prime} \in S_{h}^{1}\left(\Gamma_{h}\right),\left\|v_{h}^{\prime}\right\|_{H^{1 / 2}(\Gamma)}>0} \frac{\left|\left\langle D_{k} v_{h}, v_{h}^{\prime}\right\rangle_{\Gamma}\right|}{\left\|v_{h}^{\prime}\right\|_{H^{1 / 2}(\Gamma)}} .
\end{aligned}
$$


Proof. We prove the LBB condition for the Dirichlet boundary value problem and the proof for the Neumann boundary value problem is the same. We define $B:=V_{k}+C_{V}$. $B$ is $H^{-1 / 2}(\Gamma)$-elliptic. We define a new variational problem to find $\bar{v} \in H^{-1 / 2}(\Gamma)$ such that

$$
\langle B \bar{v}, v\rangle_{\Gamma}=\left\langle C_{V} \chi_{h}, v\right\rangle_{\Gamma} \quad \forall v \in H^{-1 / 2}(\Gamma) .
$$

We have $\bar{v}=B^{-1} C_{V} \chi_{h}$. The Galerkin formulation is to find $\bar{v}_{h} \in S_{h}^{0}\left(\Gamma_{h}\right)$ such that

$$
\left\langle B \bar{v}_{h}, v_{h}\right\rangle_{\Gamma}=\left\langle C_{V} \chi_{h}, v_{h}\right\rangle_{\Gamma} \quad \forall v_{h} \in S_{h}^{0}\left(\Gamma_{h}\right) .
$$

From the variational formulation and the Galerkin formulation we get

$$
\left\langle B \bar{v}_{h}-B \bar{v}, v_{h}\right\rangle_{\Gamma}=0 \quad \forall v_{h} \in S_{h}^{0}\left(\Gamma_{h}\right) .
$$

This defines a Galerkin projection $G_{h B}: \bar{v} \rightarrow \bar{v}_{h}$ and $G_{h B} \rightarrow I$ as $h \rightarrow 0$.

$V_{k}$ and $B$ are bounded. $B$ is $H^{-1 / 2}(\Gamma)$-elliptic and $V_{k}$ satisfies the Gårding inequality. Then we have $B$ and $V_{k}$ are invertible, and $B^{-1}$ and $V_{k}^{-1}$ are bounded. We define two operators by $G_{h B}, B$ and $C_{V}$,

$$
L:=I-B^{-1} C_{V}=B^{-1} V_{k} \quad \text { and } \quad L_{h}:=I-G_{h B} B^{-1} C_{V} .
$$

By the properties of $G_{h B}, B$ and $C_{V}, L$ is bounded and invertible, $L^{-1}$ is bounded and $L_{h}$ is bounded. By the consistency of $G_{h B}$ we get

$$
\left\|L-L_{h}\right\|=\left\|\left(G_{h B}-I\right) B^{-1} C_{V}\right\| \rightarrow 0 \quad \text { as } h \rightarrow 0 .
$$

So $L_{h}^{-1}$ exits and it is uniformly bounded if $h$ is as small as enough.

We begin to consider the right-hand side of (1.2.20a).

$$
\begin{gathered}
\left\langle V_{k} \chi_{h}, \chi_{h}^{\prime}\right\rangle_{\Gamma}=\left\langle B L_{h} \chi_{h}, \chi_{h}^{\prime}\right\rangle_{\Gamma}-\left\langle\left(B L_{h}-B L\right) \chi_{h}, \chi_{h}^{\prime}\right\rangle_{\Gamma} \\
\left|\left\langle V_{k} \chi_{h}, \chi_{h}^{\prime}\right\rangle_{\Gamma}\right|+\left|\left\langle\left(B L_{h}-B L\right) \chi_{h}, \chi_{h}^{\prime}\right\rangle_{\Gamma}\right| \geq\left|\left\langle B L_{h} \chi_{h}, \chi_{h}^{\prime}\right\rangle_{\Gamma}\right|
\end{gathered}
$$

If $\chi_{h}^{\prime}=L_{h} \chi_{h}$ we have

$$
\left\langle B L_{h} \chi_{h}, L_{h} \chi_{h}\right\rangle_{\Gamma} \geq c_{1}\left\|L_{h} \chi_{h}\right\|_{H^{-1 / 2}(\Gamma)}^{2} \geq c_{1} c_{2}\left\|\chi_{h}\right\|_{H^{-1 / 2}(\Gamma)}\left\|L_{h} \chi_{h}\right\|_{H^{-1 / 2}(\Gamma)}
$$

by using $L_{h}^{-1}$ is uniformly bounded in the last inequality and

$$
\left|\left\langle\left(B L_{h}-B L\right) \chi_{h}, L_{h} \chi_{h}\right\rangle_{\Gamma}\right| \leq\|B\|\left\|L_{h}-L\right\|\left\|\chi_{h}\right\|_{H^{-1 / 2}(\Gamma)}\left\|L_{h} \chi_{h}\right\|_{H^{-1 / 2}(\Gamma)} .
$$

Then we have

$$
\left|\left\langle V_{k} \chi_{h}, L_{h} \chi_{h}\right\rangle_{\Gamma}\right| \geq\left(c_{1} c_{2}-\|B\|\left\|L_{h}-L\right\|\right)\left\|\chi_{h}\right\|_{H^{-1 / 2}(\Gamma)}\left\|L_{h} \chi_{h}\right\|_{H^{-1 / 2}(\Gamma)} .
$$

We can choose $h$ as small as enough such that $c_{1} c_{2}-\|B\|\left\|L_{h}-L\right\|>c_{s}$ and the proof is done.

From the BBL condition it is easy to get the stability of (1.2.18) and (1.2.19). For a 
finite dimensional problem, uniqueness implies existence. So by the stability we have the existence and uniqueness of the solutions of (1.2.18) and (1.2.19).

Lemma 5. There exists $c_{1}>0$ and $c_{2}>0$ such that for the solutions of (1.2.14) and (1.2.15) and the solutions of (1.2.18) and (1.2.19), we have

$$
\begin{gathered}
\left\|\sigma-\sigma_{h}\right\|_{H^{-1 / 2}(\Gamma)} \leq c_{1} \inf _{\chi_{h} \in S_{h}^{0}\left(\Gamma_{h}\right)}\left\|\sigma-\chi_{h}\right\|_{H^{-1 / 2}(\Gamma)}, \\
\left\|\varphi-\varphi_{h}\right\|_{H^{1 / 2}(\Gamma)} \leq c_{2} \inf _{v_{h} \in S_{h}^{1}\left(\Gamma_{h}\right)}\left\|\varphi-v_{h}\right\|_{H^{1 / 2}(\Gamma)} .
\end{gathered}
$$

Proof. We prove Cea's lemma for the Dirichlet boundary value problem following [65, Theorem 8.10] and the proof for the Neumann boundary value problem is the same. From (1.2.14) and (1.2.18) we have

$$
\left\langle V_{k} \sigma_{h}, \chi_{h}\right\rangle_{\Gamma}=\left\langle V_{k} \sigma, \chi_{h}\right\rangle_{\Gamma} \quad \text { for all } \chi_{h} \in S_{h}^{0}\left(\Gamma_{h}\right) .
$$

This defines a Galerkin projection $G_{h V_{k}} \sigma=\sigma_{h}$. By the stability from the LBB condition, $G_{h V_{k}}$ is bounded. Then we have

$$
\begin{aligned}
\left\|\sigma-\sigma_{h}\right\|_{H^{-1 / 2}(\Gamma)} & =\left\|\sigma-G_{h V_{k}} \chi_{h}+G_{h V_{k}} \chi_{h}-\sigma_{h}\right\|_{H^{-1 / 2}(\Gamma)} \\
& \leq\left\|\sigma-\chi_{h}\right\|_{H^{-1 / 2}(\Gamma)}+\left\|G_{h V_{k}}\left(\chi_{h}-\sigma\right)\right\|_{H^{-1 / 2}(\Gamma)} \\
& \leq(1+c)\left\|\sigma-\chi_{h}\right\|_{H^{-1 / 2}(\Gamma)}
\end{aligned}
$$

The proof is done.

We need the following approximation properties of $S_{h}^{0}\left(\Gamma_{h}\right)$ and $S_{h}^{1}\left(\Gamma_{h}\right)$ for $\chi \in$ $H_{p w}^{s}(\Gamma)$ and $v \in H_{p w}^{s}(\Gamma)$, respectively, from [58, Theorem 2.1, 2.3] for quasi-optimal error estimates. $H_{p w}^{s}(\Gamma):=\left\{u \in L^{2}(\Gamma)|u|_{\Gamma_{i}} \in H^{s}\left(\Gamma_{i}\right), i=1, \cdots, n\right\}$.

$$
\begin{array}{cl}
\inf _{\chi_{h} \in S_{h}^{0}\left(\Gamma_{h}\right)}\left\|\chi-\chi_{h}\right\|_{H^{a}(\Gamma)} \leq c h^{s-a}|\chi|_{H_{p w}^{s}(\Gamma)} & \text { for } a \in[-1,0] \text { and } s \in[0,1], \\
\inf _{v_{h} \in S_{h}^{1}\left(\Gamma_{h}\right)}\left\|v-v_{h}\right\|_{H^{a}(\Gamma)} \leq c h^{s-a}|v|_{H_{p w}^{s}(\Gamma)} \quad \text { for } a \in[-2,1] \text { and } s \in[1,2] .
\end{array}
$$

We use (1.2.22a) and (1.2.22b) in (1.2.21a) and (1.2.21b), respectively, and get quasioptimal error estimates

$$
\begin{aligned}
\left\|\sigma-\sigma_{h}\right\|_{H^{-1 / 2}(\Gamma)} & \leq c h^{s+1 / 2}|\sigma|_{H_{p w}^{s}(\Gamma)}, \\
\left\|\varphi-\varphi_{h}\right\|_{H^{1 / 2}(\Gamma)} & \leq c h^{s-1 / 2}|\varphi|_{H_{p w}^{s}(\Gamma)} .
\end{aligned}
$$

From (1.2.23a) and (1.2.23b), it is obviously that it is difficult to calculate error in the norms, $\|\cdot\|_{H^{-1 / 2}(\Gamma)}$ and $\|\cdot\|_{H^{1 / 2}(\Gamma)}$. A good choice is to use $L^{2}(\Gamma)$-norm. We use Aubin-Nitsche duality to derive error estimates in $L^{2}(\Gamma)$-norm.

Theorem 2. Let $\sigma \in H_{p w}^{s}(\Gamma)$ for $s \in[0,1]$ and $\varphi \in H_{p w}^{s}(\Gamma)$ for $s \in[1,2]$. We have

$$
\begin{aligned}
\left\|\sigma-\sigma_{h}\right\|_{L^{2}(\Gamma)} & \leq c h^{s}|\sigma|_{H_{p w}^{s}(\Gamma)}, \\
\left\|\varphi-\varphi_{h}\right\|_{L^{2}(\Gamma)} & \leq c h^{s}|\varphi|_{H_{p w}^{s}(\Gamma)} .
\end{aligned}
$$


Proof. We prove the error estimate for the Dirichlet boundary value problem following [65, Lemma 12.2] and the proof for the Neumann boundary value problem is the same. In the proof we need the global inverse inequality from [65, Lemma 10.10] and the approximation properties of $L^{2}$-projection in $S_{h}^{0}\left(\Gamma_{h}\right)$. Let $Q_{h} \sigma$ be $L^{2}$-projection of $\sigma$ to $S_{h}^{0}\left(\Gamma_{h}\right)$.

$$
\begin{aligned}
&\left\|\sigma-\sigma_{h}\right\|_{L^{2}(\Gamma)} \leq\left\|\sigma-Q_{h} \sigma\right\|_{L^{2}(\Gamma)}+\left\|Q_{h} \sigma-\sigma_{h}\right\|_{L^{2}(\Gamma)} \\
& \underbrace{\leq}_{\text {global inverse inequality }}\left\|\sigma-Q_{h} \sigma\right\|_{L^{2}(\Gamma)}+c h^{-1 / 2}\left\|Q_{h} \sigma-\sigma_{h}\right\|_{H^{-1 / 2}(\Gamma)} \\
& \leq\left\|\sigma-Q_{h} \sigma\right\|_{L^{2}(\Gamma)}+c h^{-1 / 2}\left(\left\|Q_{h} \sigma-\sigma\right\|_{H^{-1 / 2}(\Gamma)}+\left\|\sigma-\sigma_{h}\right\|_{H^{-1 / 2}(\Gamma)}\right)
\end{aligned}
$$

By the approximation properties of $L^{2}$-projection in $L^{2}(\Gamma)$-norm and $H^{-1 / 2}(\Gamma)$-norm from [65, Theorem 10.2] and [65, Corollary 10.3], respectively, and (1.2.23a), the proof is done.

\subsubsection{Numerical tests}

We consider two numerical tests on the boundary of a unit cube $(0,1)^{3}$. In the first test we assume that $k=0$ and (1.2.1) is the Laplace equation. An analytical solution used for the test is

$$
u_{0}(x)=\left(1+x_{1}\right) \mathrm{e}^{x_{2}} \cos \left(x_{3}\right) .
$$

In the second test we consider the Helmholtz equation $(k=\sqrt{3})$ with an analytical solution used for the test

$$
u_{1}(x)=\left(1+x_{1}\right) \mathrm{e}^{x_{2}} \sin \left(2 x_{3}\right) .
$$

For Neumann boundary value problems of the Laplace equation, since $D$ is $H^{1 / 2}(\Gamma)$ semi-elliptic, we need additional terms in the variational formulation. In Tables 1.1 and 1.2 the first column is the level of meshes, the second column is the number of degrees of freedom, the third and fifth columns are $L^{2}$-error for the Laplace equation and Helmholtz equation respectively and the fourth and sixth columns are the rate of convergence $(s)$ in $O\left(h^{s}\right)$. Table 1.1 and Fig.1.2 are the results of Dirichlet boundary value problems of (1.2.25) and (1.2.26). Table 1.2 and Fig.1.3 are the results of Neumann boundary value problems of (1.2.25) and (1.2.26). "CR" means the rate of convergence $(s)$ and $\sigma_{h}^{n}$ is the approximated solution calculated by using a mesh of level $n$. The calculation of $L^{2}$-error and rate of convergence for $\varphi_{h}$ is similar with $\sigma_{h}$. The formulae are

$$
\begin{aligned}
\left\|\sigma_{h}-\sigma\right\|_{L^{2}(\Gamma)}^{2} & =\int_{\Gamma}\left(\sigma_{h}(x)-\sigma(x)\right)^{2} \mathrm{~d} s_{x}, \\
\mathrm{CR} & =\log _{2} \frac{\left\|\sigma_{h}^{n-1}-\sigma\right\|_{\mathbf{L}^{2}(\Gamma)}}{\left\|\sigma_{h}^{n}-\sigma\right\|_{\mathbf{L}^{2}(\Gamma)}}
\end{aligned}
$$

In the fourth and sixth columns of Table 1.1 we observe a linear convergence for piecewise constant function spaces and in the fourth and sixth columns of Table 1.2 we observe a quadratic convergence for piecewise linear function spaces approximately. 


\begin{tabular}{|c|c|c|c|c|c|}
\hline level & dof & Error $_{L}$ & $\mathrm{CR}_{L}$ & Error $_{H}$ & $\mathrm{CR}_{H}$ \\
\hline 0 & 24 & 1.38597 & - & 3.16616 & - \\
\hline 1 & 96 & 0.65702 & 1.0769 & 1.50606 & 1.0720 \\
\hline 2 & 384 & 0.28534 & 1.2033 & 0.62868 & 1.2604 \\
\hline 3 & 1536 & 0.12640 & 1.1747 & 0.26788 & 1.2307 \\
\hline
\end{tabular}

Table 1.1 Accuracy of Galerkin-BEMs for Dirichlet boundary value problems for the Laplace and Helmholtz equations

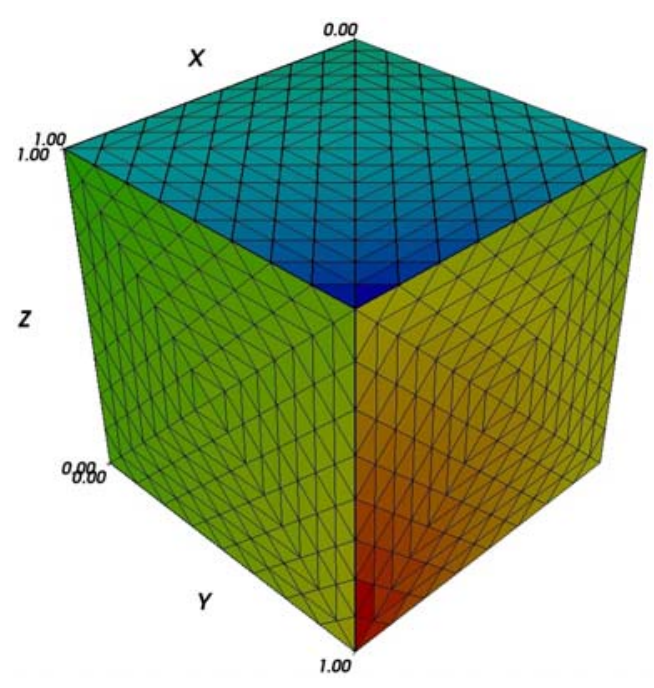

(a) the Laplace equation

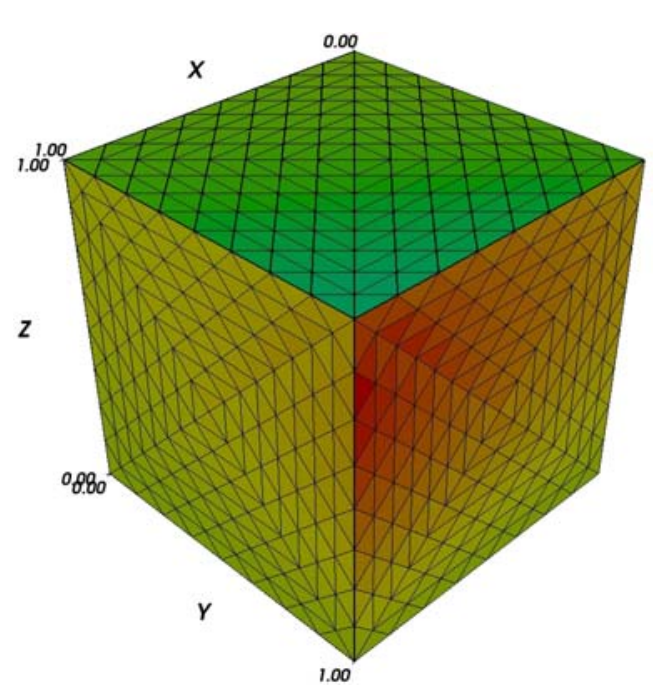

(b) the Helmholtz equation

Figure 1.2 Dirichlet boundary value problems for the Laplace and Helmholtz equations

\begin{tabular}{|c|c|c|c|c|c|}
\hline level & dof & Error $_{L}$ & $\mathrm{CR}_{L}$ & Error $_{H}$ & $\mathrm{CR}_{H}$ \\
\hline 0 & 14 & 0.39467 & - & 1.05687 & - \\
\hline 1 & 50 & 0.09237 & 2.0951 & 0.25747 & 2.0373 \\
\hline 2 & 194 & 0.02157 & 2.0984 & 0.05917 & 2.1215 \\
\hline 3 & 770 & 0.00517 & 2.0617 & 0.01413 & 2.0660 \\
\hline
\end{tabular}

Table 1.2 Accuracy of Galerkin-BEMs for Neumann boundary value problems for the Laplace and Helmholtz equations 


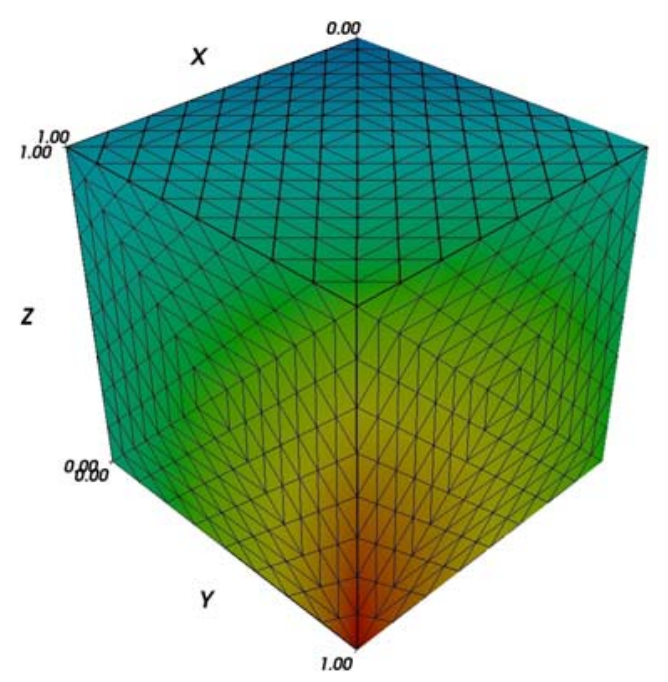

(a) the Laplace equation

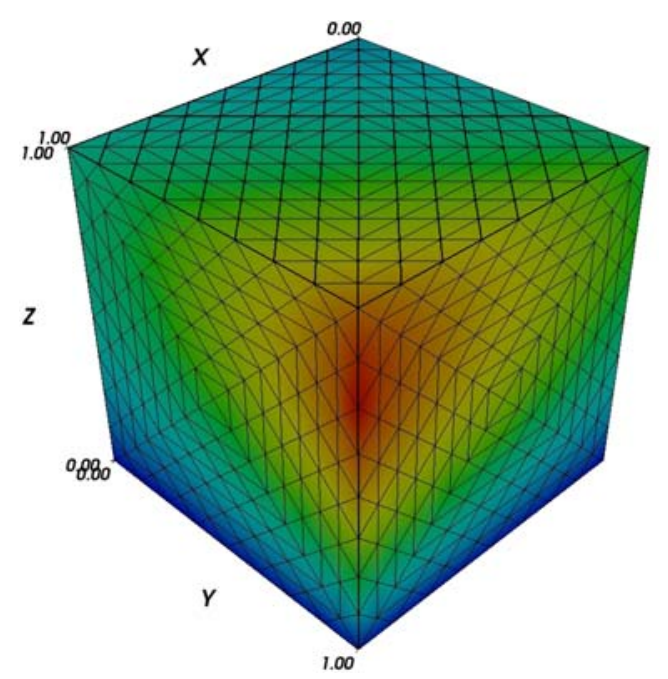

(b) the Helmholtz equation

Figure 1.3 Neumann boundary value problems for the Laplace and Helmholtz equations

\subsection{The Maxwell case}

In this section we study Dirichlet and Neumann boundary value problems for the Maxwell's equations (1.1.20). We just consider electromagnetic waves in a dielectric medium, so $\lambda$ is a real number. We assume that $\lambda=k^{2}$ and $k$ is a positive real number. The time-harmonic Maxwell's equations for electric or magnetic fields are

$$
\begin{aligned}
\nabla \times \nabla \times \mathbf{u}(x) & =k^{2} \mathbf{u}(x) & & \forall x \in \Omega, \\
\nabla \cdot \mathbf{u}(x) & =0 & & \forall x \in \Omega,
\end{aligned}
$$

where $\Omega$ is a bounded domain. Dirichlet and Neumann boundary conditions are

$$
\begin{aligned}
\mathbf{n} \times \mathbf{u}(x) & =\mathbf{f}(x) & & \forall x \in \Gamma, \\
\mathbf{n} \times \nabla \times \mathbf{u}(x) & =\mathbf{g}(x) & & \forall x \in \Gamma,
\end{aligned}
$$

respectively, where $\Gamma:=\partial \Omega, \mathbf{n}$ is the exterior unit normal to $\Gamma$, and $\mathbf{f}$ or $\mathbf{g}$ is the given data.

\subsubsection{Representation formula}

We assume that $\Omega$ has a smooth boundary. Green's first formula for (1.3.1) is

$$
\begin{aligned}
& \int_{\Omega}\left(\nabla \times \nabla \times \mathbf{u}(x)-k^{2} \mathbf{u}(x)\right) \cdot \mathbf{v}(x) \mathrm{d} x \\
& -\int_{\Omega}\left(\nabla \times \mathbf{u}(x) \cdot \nabla \times \mathbf{v}(x)-k^{2} \mathbf{u}(x) \cdot \mathbf{v}(x)\right) \mathrm{d} x \\
= & \int_{\Gamma} \mathbf{n} \times \nabla \times \mathbf{u}(x) \cdot \mathbf{v}(x) \mathrm{d} s_{x} .
\end{aligned}
$$


Green's second formula is

$$
\begin{aligned}
& \int_{\Omega}\left(\nabla \times \nabla \times \mathbf{u}(x)-k^{2} \mathbf{u}(x)\right) \cdot \mathbf{v}(x) \mathrm{d} x \\
& -\int_{\Omega} \mathbf{u}(x) \cdot\left(\nabla \times \nabla \times \mathbf{v}(x)-k^{2} \mathbf{v}(x)\right) \mathrm{d} x \\
= & \int_{\Gamma} \mathbf{n} \times \nabla \times \mathbf{u}(x) \cdot \mathbf{v}(x) \mathrm{d} s_{x}-\int_{\Gamma} \mathbf{u}(x) \cdot \mathbf{n} \times \nabla \times \mathbf{v}(x) \mathrm{d} s_{x} .
\end{aligned}
$$

Inserting (1.3.1a) in the left hand side of (1.3.4) yields

$$
\begin{aligned}
& -\int_{\Omega} \mathbf{u}(x) \cdot\left(\nabla(\nabla \cdot \mathbf{v}(x))-\nabla^{2} \mathbf{v}(x)-k^{2} \mathbf{v}(x)\right) \mathrm{d} x \\
= & -\int_{\Gamma} \mathbf{n} \cdot \mathbf{u}(x) \nabla \cdot \mathbf{v}(x) \mathrm{d} s_{x}+\int_{\Omega} \mathbf{u}(x) \cdot\left(\nabla^{2} \mathbf{v}(x)+k^{2} \mathbf{v}(x)\right) \mathrm{d} x .
\end{aligned}
$$

With the right hand side of (1.3.4) we get

$$
\begin{aligned}
& \int_{\Omega} \mathbf{u}(x) \cdot\left(\nabla^{2} \mathbf{v}(x)+k^{2} \mathbf{v}(x)\right) \mathrm{d} x=\int_{\Gamma} \mathbf{n} \times \nabla \times \mathbf{u}(x) \cdot \mathbf{v}(x) \mathrm{d} s_{x} \\
& +\int_{\Gamma} \mathbf{n} \cdot \mathbf{u}(x) \nabla \cdot \mathbf{v}(x) \mathrm{d} s_{x}+\int_{\Gamma} \mathbf{n} \times \mathbf{u}(x) \cdot \nabla \times \mathbf{v}(x) \mathrm{d} s_{x} .
\end{aligned}
$$

We set $\mathbf{v}=E_{k}(x, y) \mathbf{e}_{i}$ in (1.3.5) with $i=1,2,3 . \mathbf{e}_{i}$ is a unit vector. $E_{k}(x, y)$ is the fundamental solution (1.2.5) of the Helmholtz equation. We exchange the notation for $x$ and $y$ and get

$$
\begin{aligned}
\mathbf{u}_{i}(x)= & -\int_{\Gamma} \mathbf{n}_{y} \times \nabla_{y} \times \mathbf{u}(y) \cdot\left(E_{k}(x, y) \mathbf{e}_{i}\right) \mathrm{d} s_{y}-\int_{\Gamma} \mathbf{n}_{y} \cdot \mathbf{u}(y) \nabla_{y} \cdot\left(E_{k}(x, y) \mathbf{e}_{i}\right) \mathrm{d} s_{y} \\
& -\int_{\Gamma} \mathbf{n}_{y} \times \mathbf{u}(y) \cdot \nabla_{y} \times\left(E_{k}(x, y) \mathbf{e}_{i}\right) \mathrm{d} s_{y} \\
= & -\int_{\Gamma} \mathbf{n}_{y} \times \nabla_{y} \times \mathbf{u}(y) \cdot \mathbf{e}_{i} E_{k}(x, y) \mathrm{d} s_{y}-\int_{\Gamma} \mathbf{n}_{y} \cdot \mathbf{u}(y) \frac{\partial E_{k}(x, y)}{\partial y_{i}} \mathrm{~d} s_{y} \\
& +\int_{\Gamma} \mathbf{n}_{y} \times \mathbf{u}(y) \cdot \nabla_{x} \times\left(E_{k}(x, y) \mathbf{e}_{i}\right) \mathrm{d} s_{y} \\
= & -\int_{\Gamma} \mathbf{n}_{y} \times \nabla_{y} \times \mathbf{u}(y) \cdot \mathbf{e}_{i} E_{k}(x, y) \mathrm{d} s_{y}+\frac{\partial}{\partial x_{i}} \int_{\Gamma} \mathbf{n}_{y} \cdot \mathbf{u}(y) E_{k}(x, y) \mathrm{d} s_{y} \\
& -\nabla_{x} \times \int_{\Gamma} \mathbf{n}_{y} \times \mathbf{u}(y) \cdot E_{k}(x, y) \mathrm{d} s_{y} \cdot \mathbf{e}_{i} .
\end{aligned}
$$

We put $\mathbf{u}_{i}$ for $i=1,2,3$ together and get the Stratton-Chu representation formula

$$
\begin{aligned}
\mathbf{u}(x)= & -\int_{\Gamma} \mathbf{n}_{y} \times \nabla_{y} \times \mathbf{u}(y) \cdot E_{k}(x, y) \mathrm{d} s_{y}+\nabla_{x} \int_{\Gamma} \mathbf{n}_{y} \cdot \mathbf{u}(y) E_{k}(x, y) \mathrm{d} s_{y} \\
& -\nabla_{x} \times \int_{\Gamma} \mathbf{n}_{y} \times \mathbf{u}(y) \cdot E_{k}(x, y) \mathrm{d} s_{y} .
\end{aligned}
$$


Now we derive an alternative evaluation of $\mathbf{n}_{y} \cdot \mathbf{u}(y)$ in (1.3.6).

$$
\begin{aligned}
\int_{\Gamma} \operatorname{div}_{\Gamma}(\mathbf{n} \times \mathbf{w}(x)) v(x) \mathrm{d} s_{x} & =-\int_{\Gamma} \mathbf{n} \times \mathbf{w}(x) \cdot\left(\nabla v(x)-\mathbf{n} \cdot \frac{\partial}{\partial \mathbf{n}} v(x)\right) \mathrm{d} s_{x} \\
& =-\int_{\Omega} \nabla \times \mathbf{w}(x) \cdot \nabla v(x) \mathrm{d} x+\int_{\Omega} \mathbf{w}(x) \cdot \nabla \times \nabla v(x) \mathrm{d} x \\
& =-\int_{\Omega} \nabla \times \mathbf{w}(x) \cdot \nabla v(x) \mathrm{d} x-\int_{\Omega} \nabla \cdot \nabla \times \mathbf{w}(x) v(x) \mathrm{d} x \\
& =-\int_{\Gamma} \mathbf{n} \cdot \nabla \times \mathbf{w}(x) v(x) \mathrm{d} s_{x}
\end{aligned}
$$

From the above derivation we get

$$
\int_{\Gamma} \operatorname{div}_{\Gamma}(\mathbf{n} \times \mathbf{w}(x)) v(x) \mathrm{d} s_{x}=-\int_{\Gamma} \mathbf{n} \cdot \nabla \times \mathbf{w}(x) v(x) \mathrm{d} s_{x} .
$$

We use (1.3.7) in the second term of the right-hand side of (1.3.6) and get

$$
\begin{aligned}
\nabla_{x} \int_{\Gamma} \mathbf{n}_{y} \cdot \mathbf{u}(y) E_{k}(x, y) \mathrm{d} s_{y} & =\nabla_{x} \int_{\Gamma} \mathbf{n}_{y} \cdot \frac{\nabla_{y} \times \nabla_{y} \times \mathbf{u}(y)}{k^{2}} E_{k}(x, y) \mathrm{d} s_{y} \\
& =-\frac{1}{k^{2}} \nabla_{x} \int_{\Gamma} \operatorname{div}_{\Gamma}\left(\mathbf{n}_{y} \times \nabla_{y} \times \mathbf{u}(y)\right) E_{k}(x, y) \mathrm{d} s_{y}
\end{aligned}
$$

Then (1.3.6) is changed to

$$
\begin{aligned}
\mathbf{u}(x)= & -\int_{\Gamma} \mathbf{n}_{y} \times \nabla_{y} \times \mathbf{u}(y) \cdot E_{k}(x, y) \mathrm{d} s_{y} \\
& -\frac{1}{k^{2}} \nabla_{x} \int_{\Gamma} \operatorname{div}_{\Gamma}\left(\mathbf{n}_{y} \times \nabla_{y} \times \mathbf{u}(y)\right) E_{k}(x, y) \mathrm{d} s_{y} \quad \forall x \in \Omega . \\
& -\nabla_{x} \times \int_{\Gamma} \mathbf{n}_{y} \times \mathbf{u}(y) \cdot E_{k}(x, y) \mathrm{d} s_{y}
\end{aligned}
$$

Since we assume that $\mathbf{u}$ is a regular solution and the boundary is a smooth boundary, we call (1.3.8) the classical representation formula for (1.3.1).

\subsubsection{Function spaces}

In Section 1.2.2 we introduce function spaces for scalar-valued functions. In this section we introduce continuously differentiable function spaces and Lebesgue integrable function spaces for vector-valued functions and use them to define the function spaces for the Maxwell's equations in the domain and on the boundary. The function spaces on the boundary have been studied in $[9,10,6]$ for piecewise smooth boundaries and in [12] for Lipschitz boundaries. [7] is a summary of all these work.

Let $\Omega$ be a bounded domain. The definitions of continuously differentiable function spaces $\mathbf{C}^{m}(\Omega)$ and Hölder continuously differentiable function spaces $\mathbf{C}^{m+\beta}(\Omega)$ are

$$
\mathbf{C}^{m}(\Omega):=\left\{\mathbf{u} \mid \mathbf{u}_{i} \in C^{m}(\Omega)\right\} \quad \text { and } \quad \mathbf{C}^{m+\beta}(\Omega):=\left\{\mathbf{u} \mid \mathbf{u}_{i} \in C^{m+\beta}(\Omega)\right\} .
$$


The definition of $\mathbf{L}^{2}(\Omega)$ is

$$
\mathbf{L}^{2}(\Omega):=\left\{\mathbf{u} \mid \mathbf{u}_{i} \in L^{2}(\Omega)\right\} \quad \text { with } \quad\|\mathbf{u}\|_{\mathbf{L}^{2}(\Omega)}^{2}:=\sum_{i=1}^{3}\left\|\mathbf{u}_{i}\right\|_{L^{2}(\Omega)}^{2} .
$$

The definitions of $\mathbf{H}^{m}(\Omega)$ and $\mathbf{H}^{m+\beta}(\Omega)$ for $m \in \mathbb{N}^{0}$ and $\beta \in(0,1)$ are

$$
\begin{aligned}
& \mathbf{H}^{m}(\Omega):=\overline{\mathbf{C}^{\infty}(\Omega)}\|\cdot\|_{\mathbf{H}^{m}(\Omega)} \quad \text { with } \quad\|\mathbf{u}\|_{\mathbf{H}^{m}(\Omega)}^{2}:=\sum_{i=1}^{3}\left\|\mathbf{u}_{i}\right\|_{H^{m}(\Omega)}^{2}, \\
& \mathbf{H}^{m+\beta}(\Omega):=\overline{\mathbf{C}^{\infty}(\Omega)}\|\cdot\|_{\mathbf{H}^{m+\beta}(\Omega)} \quad \text { with } \quad\|\mathbf{u}\|_{\mathbf{H}^{m+\beta}(\Omega)}^{2}:=\sum_{i=1}^{3}\left\|\mathbf{u}_{i}\right\|_{H^{m+\beta}(\Omega)}^{2} .
\end{aligned}
$$

Let $\mathbf{d}$ be a first order differential operator. The definitions of $\mathbf{H}^{m}(\mathbf{d}, \Omega)$ and $\mathbf{H}^{m+\beta}(\mathbf{d}, \Omega)$ are given by

$$
\begin{gathered}
\mathbf{H}^{m}(\mathbf{d}, \Omega):=\left\{\mathbf{u} \in \mathbf{H}^{m}(\Omega) \mid \mathbf{d} \mathbf{u} \in \mathbf{H}^{m}(\Omega)\right\} \\
\text { with } \quad\|\mathbf{u}\|_{\mathbf{H}^{m}(\mathbf{d}, \Omega)}^{2}:=\|\mathbf{u}\|_{\mathbf{H}^{m}(\Omega)}^{2}+\|\mathbf{d} \mathbf{u}\|_{\mathbf{H}^{m}(\Omega)}^{2}, \\
\mathbf{H}^{m+\beta}(\mathbf{d}, \Omega):=\left\{\mathbf{u} \in \mathbf{H}^{m+\beta}(\Omega) \mid \mathbf{d} \mathbf{u} \in \mathbf{H}^{m+\beta}(\Omega)\right\} \\
\text { with } \quad\|\mathbf{u}\|_{\mathbf{H}^{m+\beta}(\mathbf{d}, \Omega)}^{2}:=\|\mathbf{u}\|_{\mathbf{H}^{m+\beta}(\Omega)}^{2}+\|\mathbf{d} \mathbf{u}\|_{\mathbf{H}^{m+\beta}(\Omega)}^{2} .
\end{gathered}
$$

Let $\mathbf{H}(\Omega)$ denote $\mathbf{L}^{2}(\Omega)$. The common Sobolev spaces in the study of the Maxwell's equations are $\mathbf{H}(\operatorname{curl}, \Omega), \mathbf{H}(\operatorname{div}, \Omega)$ and $\mathbf{H}\left(\operatorname{curl}^{2}, \Omega\right)$.

For boundary integral equations we need function spaces defined on the manifold $\Gamma$. One common way is to use the Dirichlet trace to define function spaces on $\Gamma$. In Section 1.2.2 the Sobolev space $H^{m+\beta}(\Gamma)$ is defined as the trace space of $H^{m+\beta+1 / 2}(\Omega)$ by the Dirichlet trace operator $\gamma_{0}$ and $H^{-m-\beta}(\Gamma)$ is the dual space of $H^{m+\beta}(\Gamma)$. According the boundary condition (1.3.2a) we use the tangential trace $\left.(\mathbf{n} \times \mathbf{u})\right|_{\Gamma}$ as the Dirichlet trace for the Maxwell's equations. We compare $\left.(\mathbf{n} \times \mathbf{u})\right|_{\Gamma}$ with $\gamma_{0} u$. If the boundary is a smooth boundary, we have the definition of $\mathbf{n}$ on the whole boundary. We can use Sobolev spaces on $\Gamma$ in Section 1.2.2 to define Sobolev spaces on $\Gamma$ for the Maxwell's equations. More discussions about smooth boundaries could be found in [55, 2, 15, 52]. If the boundary is a non-smooth boundary, we don't have the definition of $\mathbf{n}$ on the whole boundary. So even if $\mathbf{u} \in \mathbf{C}^{\infty}(\bar{\Omega})$, we don't have $\left.(\mathbf{n} \times \mathbf{u})\right|_{\Gamma} \in \mathbf{H}^{1 / 2}(\Gamma)$. We can not use Sobolev spaces on $\Gamma$ in Section 1.2.2, so we define new Hilbert spaces on $\Gamma$ by the tangential trace

$$
\mathbf{H}_{\times}^{\beta}(\Gamma):=\left\{\left.(\mathbf{n} \times \mathbf{u})\right|_{\Gamma} \mid \mathbf{u} \in \mathbf{H}^{\beta+1 / 2}(\Omega)\right\} \quad \forall \beta \in(0,1),
$$

with an inner product such that the tangential trace is continuous and surjective. The definition of the tangential trace is generalized to a weak solution of

$$
\int_{\Omega} \nabla \times \mathbf{u}(x) \cdot \mathbf{v}(x) \mathrm{d} x-\int_{\Omega} \mathbf{u}(x) \cdot \nabla \times \mathbf{v}(x) \mathrm{d} x=\int_{\Gamma} \mathbf{n}(x) \times \mathbf{u}(x) \cdot \mathbf{v}(x) \mathrm{d} s_{x} .
$$

We define

$$
\mathbf{L}_{\mathbf{t}}^{2}(\Gamma):=\left\{\mathbf{u} \in \mathbf{L}^{2}(\Gamma) \mid \mathbf{n} \cdot \mathbf{u}=0\right\}
$$


$\mathbf{H}_{\times}^{-\beta}(\Gamma)$ is the dual space of $\mathbf{H}_{\times}^{\beta}(\Gamma)$ with $\mathbf{L}_{\mathbf{t}}^{2}(\Gamma)$ as a pivot space. We use the following dual pairing

$$
\langle\mathbf{v}, \mathbf{w}\rangle_{\tau, \Gamma}:=\int_{\Gamma}(\mathbf{v} \times \mathbf{n})(x) \cdot \mathbf{w}(x) \mathrm{d} s_{x} .
$$

More discussions about non-smooth boundaries see [6, 9, 10, 12].

For curvilinear polyhedra, a norm is given in [9] for $\mathbf{H}_{\times}^{1 / 2}(\Gamma)$. Let $\Gamma:=\bigcup_{i=1}^{n} \bar{\Gamma}_{i}$. $I_{i}$ is the set of indices of $\Gamma_{j}$ such that $\bar{\Gamma}_{j} \cap \bar{\Gamma}_{i} \neq 0$ and $i \neq j . \mathbf{n}_{i j}$ is the exterior tangential normal to $\Gamma_{i}$ and on $\bar{\Gamma}_{i} \bigcap \bar{\Gamma}_{j}$. The norm is defined by

$$
\begin{aligned}
\|\mathbf{v}\|_{\mathbf{H}_{\times}^{1 / 2}(\Gamma)}^{2}:= & \sum_{i=1}^{n}\left\|\left.\mathbf{v}\right|_{\Gamma_{i}}\right\|_{\mathbf{H}^{1 / 2}\left(\Gamma_{i}\right)}^{2} \\
& +\sum_{i=1}^{n} \sum_{j \in I_{i}} \int_{\Gamma_{i}} \int_{\Gamma_{j}} \frac{|\mathbf{v}|_{\Gamma_{i}}(x) \cdot \mathbf{n}_{i j}(x)-\left.\left.\mathbf{v}\right|_{\Gamma_{j}}(y) \cdot \mathbf{n}_{j i}(y)\right|^{2}}{|x-y|^{3}} \mathrm{~d} s_{y} \mathrm{~d} s_{x} .
\end{aligned}
$$

The $\operatorname{div}_{\Gamma}$ in (1.3.7) is generalized in $[6,8,12]$ to

$$
\operatorname{div}_{\Gamma} \mathbf{v}:=\left\{\begin{array}{ll}
\operatorname{div}_{\Gamma_{i}}\left(\left.\mathbf{v}\right|_{\Gamma_{i}}\right) & \text { on } \Gamma_{i} \\
\left.\mathbf{v}\right|_{\Gamma_{i}} \cdot \mathbf{n}_{i j}+\left.\mathbf{v}\right|_{\Gamma_{j}} \cdot \mathbf{n}_{j i} & \text { on } \bar{\Gamma}_{i} \bigcap \bar{\Gamma}_{j}
\end{array} .\right.
$$

Then we could define the Hilbert space on $\Gamma$ for the Maxwell's equations

$$
\mathbf{H}_{\times}^{-1 / 2}\left(\operatorname{div}_{\Gamma}, \Gamma\right):=\left\{\mathbf{v} \in \mathbf{H}_{\times}^{-1 / 2}(\Gamma) \mid \operatorname{div}_{\Gamma} \mathbf{v} \in H^{-1 / 2}(\Gamma)\right\}
$$

\subsubsection{Boundary integral equations}

When we derive the classical representation formula, we assume that the boundary is smooth and $\mathbf{u} \in \mathbf{C}^{2}(\Omega)$. In the last section we have introduced (2a) in Fig. 1.1 and in this section we continue to introduce $(2 b)$ and $(2 c)$, then we can generalize our problems from (1.3.8). We simplify the notation from $\mathbf{H}_{\times}^{-1 / 2}\left(\operatorname{div}_{\Gamma}, \Gamma\right)$ to $\mathbf{W}^{-1 / 2}(\Gamma)$.

Let $\mathbf{u}$ be the solution of (1.3.1). According the Dirichlet and Neumann boundary conditions (1.3.2a) and (1.3.2b), the classical definitions of trace operators are given by

$\gamma_{\mathbf{t}}(\mathbf{u})(x):=\lim _{\tilde{x} \in \Omega \rightarrow x \in \Gamma} \mathbf{n}(x) \times \mathbf{u}(\tilde{x}) \quad$ and $\quad \gamma_{\mathbf{N}}(\mathbf{u})(x):=\lim _{\tilde{x} \in \Omega \rightarrow x \in \Gamma} \mathbf{n}(x) \times \nabla \times \mathbf{u}(\tilde{x})$.

We need a generalized definition of $\gamma_{\mathbf{N}}(\mathbf{u})$ as the weak solution of the following variational formulation

$$
\left\langle\gamma_{\mathbf{N}}(\mathbf{u}), \gamma_{\mathbf{t}}(\mathbf{v})\right\rangle_{\tau, \Gamma}:=\frac{1}{k} \int_{\Omega}(\nabla \times \nabla \times \mathbf{u}(x) \cdot \mathbf{v}(x)-\nabla \times \mathbf{u}(x) \cdot \nabla \times \mathbf{v}(x)) \mathrm{d} x,
$$

for $\mathbf{u} \in \mathbf{H}\left(\operatorname{curl}^{2}, \Omega\right)$ and $\mathbf{v} \in \mathbf{C}^{\infty}(\Omega)$. The generalized definition of $\gamma_{\mathbf{t}}(\mathbf{u})$ is given in the last section. We call $\gamma_{\mathbf{t}}$ the Dirichlet trace operator and $\gamma_{\mathbf{N}}$ the Neumann trace operator. We have a lemma for the continuity of the trace operators.

Lemma 6. Let $\Gamma$ be a Lipschitz boundary. The Dirichlet trace operator $\gamma_{\mathrm{t}}$ and Neumann 
trace operator $\gamma_{\mathbf{N}}$ are bounded operators

$$
\begin{aligned}
\gamma_{\mathbf{t}} & : \mathbf{H}(\operatorname{curl}, \Omega) \rightarrow \mathbf{W}^{-1 / 2}(\Gamma), \\
\gamma_{\mathbf{N}} & : \mathbf{H}\left(\operatorname{curl}^{2}, \Omega\right) \rightarrow \mathbf{W}^{-1 / 2}(\Gamma) .
\end{aligned}
$$

Proof. See [10, Theorem 4.4], [12, Section 4] and [13, Lemma 3].

We define two potential operators corresponding to the two boundary integrals in (1.3.8) for $\mathbf{v}, \mathbf{w} \in \mathbf{W}^{-1 / 2}(\Gamma)$ as

$$
\begin{array}{ll}
\Psi_{S L}^{k}(\mathbf{v})(x):=-k \int_{\Gamma} \mathbf{v}(y) \cdot E_{k}(x, y) \mathrm{d} s_{y}-\frac{1}{k} \nabla_{x} \int_{\Gamma} \operatorname{div}_{\Gamma}(\mathbf{v}(y)) E_{k}(x, y) \mathrm{d} s_{y} & \forall x \in \Omega, \\
\Psi_{D L}^{k}(\mathbf{w})(x):=-\nabla_{x} \times \int_{\Gamma} \mathbf{w}(y) \cdot E_{k}(x, y) \mathrm{d} s_{y} & \forall x \in \Omega .
\end{array}
$$

We call $\Psi_{S L}^{k}$ the single-layer potential operator and $\Psi_{D L}^{k}$ the double-layer potential operator. Let $\mathbf{d}$ be a first order differential operator. We define

$$
\mathbf{H}(\mathbf{d} 0, \Omega):=\{\mathbf{u} \in \mathbf{H}(\Omega) \mid \mathbf{d} \mathbf{u}=0\} .
$$

We have a lemma for the continuity of $\Psi_{S L}^{k}$ and $\Psi_{D L}^{k}$.

Lemma 7. Let $\Gamma$ be a Lipschitz boundary and let $\mathbf{v}, \mathbf{w} \in \mathbf{W}^{-1 / 2}(\Gamma)$ be given. Then, $\mathbf{u}(x)=\Psi_{S L}^{k}(\mathbf{v})(x)$ or $\mathbf{u}(x)=\Psi_{D L}^{k}(\mathbf{w})(x)$ for $x \in \Omega$ is a weak solution of the Maxwell's equations. The potential operators, $\Psi_{S L}^{k}$ and $\Psi_{D L}^{k}$, are bounded operators

$$
\begin{gathered}
\Psi_{S L}^{k}: \mathbf{W}^{-1 / 2}(\Gamma) \rightarrow \mathbf{H}\left(\mathbf{c u r l}^{2}, \Omega\right) \bigcap \mathbf{H}(\operatorname{div} 0, \Omega), \\
\Psi_{D L}^{k}: \mathbf{W}^{-1 / 2}(\Gamma) \rightarrow \mathbf{H}\left(\mathbf{c u r l}^{2}, \Omega\right) \bigcap \mathbf{H}(\operatorname{div} 0, \Omega) .
\end{gathered}
$$

Proof. See Theorem 5 in [13].

By using trace operators to potential operators, respectively, we get boundary integral operators and the continuity of boundary integral operators is from the continuity of trace operators and potential operators. For the Maxwell's equations we just need two boundary integral operators. If we compare the Dirichlet trace operator $\left(\gamma_{\mathbf{t}}\right)$ with the Neumann trace operator $\left(\gamma_{\mathbf{N}}\right)$, we find that the difference is the curl operator. If $\gamma_{\mathbf{t}}$ is the tangential trace of electric fields, $\gamma_{\mathbf{N}}$ is the tangential trace of magnetic fields. Electric fields and magnetic fields are symmetric. This is the physical explanation for two boundary integral operators. For mathematics, we can derive that the curl of $\Psi_{S L}^{k}$ is changed to $\Psi_{D L}^{k}$ and the curl of $\Psi_{D L}^{k}$ is changed to $\Psi_{S L}^{k}$. Compared with the four boundary integral operators of the Helmholtz equation, mathematics match physics very well. Let $x$ be in the domain.

$$
\begin{aligned}
\nabla_{x} \times \Psi_{S L}^{k}(\mathbf{v})(x)= & -\nabla_{x} \times\left(k \int_{\Gamma} \mathbf{v}(y) \cdot E_{k}(x, y) \mathrm{d} s_{y}\right) \\
& -\nabla_{x} \times\left(\frac{1}{k} \nabla_{x} \int_{\Gamma} \operatorname{div}_{\Gamma}(\mathbf{v}(y)) E_{k}(x, y) \mathrm{d} s_{y}\right) \\
= & -\nabla_{x} \times\left(k \int_{\Gamma} \mathbf{v}(y) \cdot E_{k}(x, y) \mathrm{d} s_{y}\right)=k \Psi_{D L}^{k}(\mathbf{v})(x)
\end{aligned}
$$




$$
\begin{aligned}
& \nabla_{x} \times \mathbf{\Psi}_{D L}^{k}(\mathbf{w})(x)=-\nabla_{x} \times\left(\nabla_{x} \times \int_{\Gamma} \mathbf{w}(y) \cdot E_{k}(x, y) \mathrm{d} s_{y}\right) \\
= & -\int_{\Gamma} \nabla_{x}\left(\nabla_{x} \cdot\left(\mathbf{w}(y) \cdot E_{k}(x, y)\right)\right) \mathrm{d} s_{y}+\int_{\Gamma} \nabla_{x}^{2}\left(\mathbf{w}(y) \cdot E_{k}(x, y)\right) \mathrm{d} s_{y} \\
= & \nabla_{x} \int_{\Gamma} \mathbf{w}(y) \cdot\left(\nabla_{y} E_{k}(x, y)-\mathbf{n}_{y} \cdot \frac{\partial E_{k}(x, y)}{\partial \mathbf{n}_{y}}\right) \mathrm{d} s_{y}+\int_{\Gamma} \mathbf{w}(y) \cdot \Delta_{y} E_{k}(x, y) \mathrm{d} s_{y} \\
= & -\nabla_{x} \int_{\Gamma} \operatorname{div}_{\Gamma}(\mathbf{w}(y)) E_{k}(x, y) \mathrm{d} s_{y}-k^{2} \int_{\Gamma} \mathbf{w}(y) \cdot E_{k}(x, y) \mathrm{d} s_{y}=k \mathbf{\Psi}_{S L}^{k}(\mathbf{w})(x)
\end{aligned}
$$

From the above derivation we get

$$
\nabla_{x} \times \Psi_{S L}^{k}(\mathbf{v})=k \Psi_{D L}^{k}(\mathbf{v}) \quad \text { and } \quad \nabla_{x} \times \Psi_{D L}^{k}(\mathbf{w})=k \Psi_{S L}^{k}(\mathbf{w}) .
$$

Theorem 3. Let $\Gamma$ be a Lipschitz boundary. Then, $\gamma_{\mathbf{t}} \boldsymbol{\Psi}_{S L}^{k}=\gamma_{\mathbf{N}} \boldsymbol{\Psi}_{D L}^{k}$ and $\gamma_{\mathbf{t}} \boldsymbol{\Psi}_{D L}^{k}=$ $\gamma_{\mathbf{N}} \Psi_{S L}^{k}$ from (1.3.10) are bounded operators

$$
\begin{gathered}
\gamma_{\mathbf{t}} \boldsymbol{\Psi}_{S L}^{k}: \mathbf{W}^{-1 / 2}(\Gamma) \rightarrow \mathbf{W}^{-1 / 2}(\Gamma), \\
\gamma_{\mathbf{t}} \boldsymbol{\Psi}_{D L}^{k}: \mathbf{W}^{-1 / 2}(\Gamma) \rightarrow \mathbf{W}^{-1 / 2}(\Gamma) .
\end{gathered}
$$

Proof. By Lemma 6 and Lemma 7.

In the calculation we need explicit formulae for boundary integral operators.

$$
\begin{aligned}
& \gamma_{\mathbf{t}} \boldsymbol{\Psi}_{S L}^{k}(\mathbf{v})(x)=-\lim _{\tilde{x} \in \Omega \rightarrow x \in \Gamma} \mathbf{n}(x) \times\left(k \lim _{\epsilon \rightarrow 0} \int_{y \in \Gamma:|y-x|>\epsilon} \mathbf{v}(y) \cdot E_{k}(\tilde{x}, y) \mathrm{d} s_{y}\right) \\
& -\lim _{\tilde{x} \in \Omega \rightarrow x \in \Gamma} \mathbf{n}(x) \times\left(\frac{1}{k} \lim _{\epsilon \rightarrow 0} \nabla_{\tilde{x}} \int_{y \in \Gamma:|y-x|>\epsilon} \operatorname{div}_{\Gamma}(\mathbf{v}(y)) E_{k}(\tilde{x}, y) \mathrm{d} s_{y}\right) \\
& =-k \lim _{\epsilon \rightarrow 0} \int_{y \in \Gamma:|y-x|>\epsilon} \mathbf{n}(x) \times\left(\mathbf{v}(y) \cdot E_{k}(x, y)\right) \mathrm{d} s_{y} \\
& -\frac{1}{k} \lim _{\epsilon \rightarrow 0} \int_{y \in \Gamma:|y-x|>\epsilon} \mathbf{n}(x) \times \nabla_{x}\left(\operatorname{div}_{\Gamma}(\mathbf{v}(y)) E_{k}(x, y)\right) \mathrm{d} s_{y} \\
& =-k \int_{\Gamma \backslash\{x\}} \mathbf{n}(x) \times\left(\mathbf{v}(y) \cdot E_{k}(x, y)\right) \mathrm{d} s_{y} \\
& -\frac{1}{k} \text { p.v. } \int_{\Gamma \backslash\{x\}} \mathbf{n}(x) \times \nabla_{x}\left(\operatorname{div}_{\Gamma}(\mathbf{v}(y)) E_{k}(x, y)\right) \mathrm{d} s_{y} \\
& \gamma_{\mathbf{t}} \mathbf{\Psi}_{D L}^{k}(\mathbf{w})(x)=-\lim _{\tilde{x} \in \Omega \rightarrow x \in \Gamma} \mathbf{n}(x) \times\left(\lim _{\epsilon \rightarrow 0} \nabla_{\tilde{x}} \times \int_{y \in \Gamma:|y-x|>\epsilon} \mathbf{w}(y) \cdot E_{k}(\tilde{x}, y) \mathrm{d} s_{y}\right) \\
& =-\lim _{\epsilon \rightarrow 0} \int_{y \in \Gamma:|y-x|>\epsilon} \mathbf{n}(x) \times \nabla_{x} \times\left(\mathbf{w}(y) \cdot E_{k}(x, y)\right) \mathrm{d} s_{y} \\
& =-\lim _{\epsilon \rightarrow 0}\left(\begin{array}{c}
\int_{y \in \Gamma:|y-x|>\epsilon}\left(\mathbf{n}(x) \cdot \mathbf{w}(y) \frac{\partial E_{k}(x, y)}{\partial x_{1}}-\mathbf{w}_{1}(y) \frac{\partial E_{k}(x, y)}{\partial \mathbf{n}_{x}}\right) \\
\int_{y \in \Gamma:|y-x|>\epsilon}\left(\mathbf{n}(x) \cdot \mathbf{w}(y) \frac{\partial E_{k}(x, y)}{\partial x_{2}}-\mathbf{w}_{2}(y) \frac{\partial E_{k}(x, y)}{\partial \mathbf{n}_{x}}\right) \\
\mathrm{d} s_{y} \\
\int_{y \in \Gamma:|y-x|>\epsilon}\left(\mathbf{n}(x) \cdot \mathbf{w}(y) \frac{\partial E_{k}(x, y)}{\partial x_{3}}-\mathbf{w}_{3}(y) \frac{\partial E_{k}(x, y)}{\partial \mathbf{n}_{x}}\right) \\
\mathrm{d} s_{y}
\end{array}\right)
\end{aligned}
$$


By using the same argument as the adjoint double-layer operator in Section 1.2.3 we get

$$
\lim _{\epsilon \rightarrow 0} \int_{y \in \Gamma:|y-x|>\epsilon} \mathbf{w}_{i}(y) \frac{\partial E_{k}(x, y)}{\partial \mathbf{n}_{x}} \mathrm{~d} s_{y}=\frac{1}{2} \mathbf{w}_{i}+\text { p.v. } \int_{\Gamma \backslash\{x\}} \mathbf{w}_{i}(y) \frac{\partial E_{k}(x, y)}{\partial \mathbf{n}_{x}} \mathrm{~d} s_{y}
$$

for $i=1,2,3$. By using this result we get

$$
\gamma_{\mathbf{t}} \Psi_{D L}^{k}(\mathbf{w})(x)=\frac{1}{2} \mathbf{w}(x)-\text { p.v. } \int_{\Gamma \backslash\{x\}} \mathbf{n}(x) \times \nabla_{x} \times\left(\mathbf{w}(y) \cdot E_{k}(x, y)\right) \mathrm{d} s_{y} .
$$

Then we could define two boundary integral operators

$$
\begin{aligned}
\mathbf{S}_{k}(\mathbf{v})(x):= & -k \int_{\Gamma \backslash\{x\}} \mathbf{n}(x) \times\left(\mathbf{v}(y) \cdot E_{k}(x, y)\right) \mathrm{d} s_{y} \\
& -\frac{1}{k} \text { p.v. } \int_{\Gamma \backslash\{x\}} \mathbf{n}(x) \times \nabla_{x}\left(\operatorname{div}_{\Gamma}(\mathbf{v}(y)) E_{k}(x, y)\right) \mathrm{d} s_{y}, \\
\mathbf{C}_{k}(\mathbf{w})(x):= & - \text { p.v. } \int_{\Gamma \backslash\{x\}} \mathbf{n}(x) \times \nabla_{x} \times\left(\mathbf{w}(y) \cdot E_{k}(x, y)\right) \mathrm{d} s_{y}
\end{aligned}
$$

for $x \in \Gamma$ and $\mathbf{v}, \mathbf{w} \in \mathbf{W}^{-1 / 2}(\Gamma)$. We call $\mathbf{S}_{k}$ the single-layer boundary integral operator and $\mathbf{C}_{k}$ the double-layer boundary integral operator. The first term of $\mathbf{S}_{k}(\mathbf{v})$ is a weakly singular integral and the second term is a Cauchy principle value integral. In fact the second term is similar with the hyper-singular boundary integral operator for the Helmholtz equation and $\operatorname{div}_{\Gamma}$ is a regularization for it. $\mathbf{C}_{k}(\mathbf{w})$ is a Cauchy principle value integral. Then we have

$$
\begin{aligned}
\mathbf{S}_{k} & =\gamma_{\mathbf{t}} \Psi_{S L}^{k}: \mathbf{W}^{-1 / 2}(\Gamma) \rightarrow \mathbf{W}^{-1 / 2}(\Gamma), \\
\frac{1}{2} I+\mathbf{C}_{k} & =\gamma_{\mathbf{t}} \Psi_{D L}^{k}: \mathbf{W}^{-1 / 2}(\Gamma) \rightarrow \mathbf{W}^{-1 / 2}(\Gamma) .
\end{aligned}
$$

(1.3.11a) and (1.3.11b) are still not enough for the calculation and we have the other two formulae for their dual pairings. In the derivation of the new formulae, we need to use $\mathbf{n} \times \mathbf{w} \times \mathbf{n} \cdot \mathbf{v}=\mathbf{w} \cdot \mathbf{v}-(\mathbf{n} \cdot \mathbf{w})(\mathbf{n} \cdot \mathbf{v})$ and get

$$
\begin{aligned}
\left\langle\mathbf{S}_{k}(\mathbf{v}), \mathbf{w}\right\rangle_{\tau, \Gamma}= & -k \int_{\Gamma} \int_{\Gamma \backslash\{x\}} \mathbf{v}(y) \cdot \mathbf{w}(x) E_{k}(x, y) \mathrm{d} s_{y} \mathrm{~d} s_{x} \\
& +\frac{1}{k} \int_{\Gamma} \int_{\Gamma \backslash\{x\}} \operatorname{div}_{\Gamma}(\mathbf{v})(y) \operatorname{div}_{\Gamma}(\mathbf{w})(x) E_{k}(x, y) \mathrm{d} s_{y} \mathrm{~d} s_{x}, \\
\left\langle\mathbf{C}_{k}(\mathbf{w}), \mathbf{v}\right\rangle_{\tau, \Gamma}= & -\int_{\Gamma} \int_{\Gamma \backslash\{x\}} \nabla_{x} E_{k}(x, y) \cdot(\mathbf{w}(y) \times \mathbf{v}(x)) \mathrm{d} s_{y} \mathrm{~d} s_{x} .
\end{aligned}
$$

We use these two formulae for the assembling of matrices and vectors in Galerkin-BEMs.

Now we can go back to the classical representation formula (1.3.8). The function space of $\mathbf{u}$ is extended to $\mathbf{H}\left(\mathbf{c u r l}^{2}, \Omega\right) \bigcap \mathbf{H}(\operatorname{div} 0, \Omega)$ and we have

$$
\mathbf{u}(x)=\Psi_{S L}^{k}\left(\gamma_{\mathbf{N}} \mathbf{u}\right)(x)+\Psi_{D L}^{k}\left(\gamma_{\mathbf{t}} \mathbf{u}\right)(x) \quad \forall x \in \Omega .
$$


(1.3.12) is the generalized representation formula. We use the Dirichlet and Neumann trace operators on (1.3.12), respectively, and get two boundary integral equations

$$
\begin{aligned}
\gamma_{\mathbf{t}} \mathbf{u} & =\mathbf{S}_{k}\left(\gamma_{\mathbf{N}} \mathbf{u}\right)+\left(\frac{1}{2} I+\mathbf{C}_{k}\right)\left(\gamma_{\mathbf{t}} \mathbf{u}\right), \\
\gamma_{\mathbf{N}} \mathbf{u} & =\left(\frac{1}{2} I+\mathbf{C}_{k}\right)\left(\gamma_{\mathbf{N}} \mathbf{u}\right)+\mathbf{S}_{k}\left(\gamma_{\mathbf{t}} \mathbf{u}\right) .
\end{aligned}
$$

The idea is the same with the Helmholtz equation. If we want to calculate the solution in the domain by (1.3.12), we need to know $\left(\gamma_{\mathbf{t}} \mathbf{u}, \gamma_{\mathbf{N}} \mathbf{u}\right)$ on the whole boundary. By using (1.3.13) we define a Calderón projection as

$$
\mathcal{C}=\left(\begin{array}{cc}
\frac{1}{2} I+\mathbf{C}_{k} & \mathbf{S}_{k} \\
\mathbf{S}_{k} & \frac{1}{2} I+\mathbf{C}_{k}
\end{array}\right): \mathbf{W}^{-1 / 2}(\Gamma) \times \mathbf{W}^{-1 / 2}(\Gamma) \rightarrow \mathbf{W}^{-1 / 2}(\Gamma) \times \mathbf{W}^{-1 / 2}(\Gamma) .
$$

The Calderón projection is a coupling of the Dirichlet trace and the Neumann trace and it is important for mixed boundary value problems, Robin boundary condition and interface problems. By using Dirichlet and Neumann boundary conditions, we get two boundary integral equations

$$
\begin{aligned}
& \mathbf{S}_{k}\left(\gamma_{\mathbf{N}} \mathbf{u}\right)=\left(\frac{1}{2} I-\mathbf{C}_{k}\right) \mathbf{f} \\
& \mathbf{S}_{k}\left(\gamma_{\mathbf{t}} \mathbf{u}\right)=\left(\frac{1}{2} I-\mathbf{C}_{k}\right) \mathbf{g} .
\end{aligned}
$$

In fact (1.3.15) and (1.3.16) are equations of the same type and we just need to change the given data to get different solutions. So in the next sections we just consider the Dirichlet boundary value problem (1.3.15).

\subsubsection{Variational formulations}

In the last section we considered step (1a) in Fig. 1.1. In this section we introduce step (1b) in Fig. 1.1. We define $\boldsymbol{\sigma}:=\gamma_{\mathbf{N}} \mathbf{u}$. The variational formulation for (1.3.15) is to find $\boldsymbol{\sigma} \in \mathbf{W}^{-1 / 2}(\Gamma)$ such that

$$
\left\langle\mathbf{S}_{k}(\boldsymbol{\sigma}), \boldsymbol{\chi}\right\rangle_{\tau, \Gamma}=\left\langle\left(\frac{1}{2} I-\mathbf{C}_{k}\right) \mathbf{f}, \boldsymbol{\chi}\right\rangle_{\tau, \Gamma}
$$

for all $\chi \in \mathbf{W}^{-1 / 2}(\Gamma)$.

For the existence and uniqueness of the solution of (1.3.17) we need a Helmholtztype regular decomposition for $\mathbf{W}^{-1 / 2}(\Gamma)$ and use it to get the generalized Gårding inequality. We explain the reason for this decomposition by a physical point of view. The physical problems described by the Helmholtz equation are different from the Maxwell's equations. For example, the Helmholtz equation could be used for acoustics. In acoustics the potential energy and the kinetic energy are converted into each other and the total energy is conserved. We can find corresponding terms for both of them in the Helmholtz equation. The Maxwell's equations are used to describe electromagnetic fields. Electric 
fields and magnetic fields are symmetric as a part of electromagnetic fields and they are converted into each other. This is a kind of conversion. But just a part of electric fields is converted into magnetic fields, we need to do a decomposition to get this part and by the conversation we could get the ellipticity. We define

$$
\mathbf{H}_{\times}^{-1 / 2}\left(\operatorname{div}_{\Gamma} 0, \Gamma\right):=\left\{\mathbf{v} \in \mathbf{H}_{\times}^{-1 / 2}(\Gamma) \mid \operatorname{div}_{\Gamma} \mathbf{v}=0\right\} .
$$

Lemma 8. There exists a projection $R^{\Gamma}$ for $\mathbf{W}^{-1 / 2}(\Gamma)$ such that

$$
\begin{aligned}
R^{\Gamma}: \mathbf{W}^{-1 / 2}(\Gamma) & \rightarrow \mathbf{H}_{\times}^{1 / 2}(\Gamma), \\
Z^{\Gamma}:=I-R^{\Gamma}: \mathbf{W}^{-1 / 2}(\Gamma) & \rightarrow \mathbf{H}_{\times}^{-1 / 2}\left(\operatorname{div}_{\Gamma} 0, \Gamma\right),
\end{aligned}
$$

and we also have the continuity of $R^{\Gamma}$

$$
\left\|R^{\Gamma} \mathbf{v}\right\|_{\mathbf{W}^{-1 / 2}(\Gamma)} \leq C\left\|\operatorname{div}_{\Gamma} \mathbf{v}\right\|_{H^{-1 / 2}(\Gamma)} \quad \forall \mathbf{v} \in \mathbf{W}^{-1 / 2}(\Gamma) .
$$

Proof. See [13, Lemma 2].

We use this decomposition to prove the generalized Gårding inequality for $\mathbf{S}_{k}$.

Lemma 9. Let $\Gamma$ be a Lipschitz boundary. $X^{\Gamma}:=R^{\Gamma}-Z^{\Gamma}$. There exists a compact bilinear form $c_{\Gamma}$ such that $\mathbf{S}_{k}$ satisfies a generalized Gårding inequality

$$
\left|\left\langle\mathbf{S}_{k}(\mathbf{v}), X^{\Gamma} \mathbf{v}\right\rangle_{\tau, \Gamma}+c_{\Gamma}(\mathbf{v}, \mathbf{v})\right| \geq c_{g}\|\mathbf{v}\|_{\mathbf{W}^{-1 / 2}(\Gamma)}^{2} \quad \forall \mathbf{v} \in \mathbf{W}^{-1 / 2}(\Gamma) .
$$

Proof. The basic idea of the proof is given in [13, Lemma 10]. First we define

$$
\left\langle\mathbf{S}_{0, k}(\mathbf{v}), \mathbf{w}\right\rangle_{\tau, \Gamma}:=\frac{1}{k}\left\langle V\left(\operatorname{div}_{\Gamma}(\mathbf{v})\right), \operatorname{div}_{\Gamma}(\mathbf{w})\right\rangle_{\Gamma}-k\langle\mathbf{V}(\mathbf{v}), \mathbf{w}\rangle_{\tau, \Gamma},
$$

where $V$ is defined in Section 1.2.4, $\mathbf{V}_{k}$ is defined by the first term in (1.3.11a) and we set $\mathbf{V}=\mathbf{V}_{0}$. From [13, Corollary 4] we know that $\mathbf{S}_{0, k}-\mathbf{S}_{k}$ is compact and more details see [32, Theorem 6.2] and [19, Theorem 3]. We consider

$$
\begin{aligned}
& \left\langle\mathbf{S}_{k}(\mathbf{v}), X^{\Gamma} \mathbf{v}\right\rangle_{\tau, \Gamma}+\left\langle\mathbf{S}_{0, k}(\mathbf{v})-\mathbf{S}_{k}(\mathbf{v}), X^{\Gamma} \mathbf{v}\right\rangle_{\tau, \Gamma}=\left\langle\mathbf{S}_{0, k}\left(R^{\Gamma} \mathbf{v}+Z^{\Gamma} \mathbf{v}\right), R^{\Gamma} \mathbf{v}-Z^{\Gamma} \mathbf{v}\right\rangle_{\tau, \Gamma} \\
= & \frac{1}{k}\left\langle V\left(\operatorname{div}_{\Gamma}\left(R^{\Gamma} \mathbf{v}\right)\right), \operatorname{div}_{\Gamma}\left(R^{\Gamma} \mathbf{v}\right)\right\rangle_{\Gamma}+k\left\langle\mathbf{V}\left(Z^{\Gamma} \mathbf{v}\right), Z^{\Gamma} \mathbf{v}\right\rangle_{\tau, \Gamma}-k\left\langle\mathbf{V}\left(R^{\Gamma} \mathbf{v}\right), R^{\Gamma} \mathbf{v}\right\rangle_{\tau, \Gamma} \\
& -k\left\langle\mathbf{V}\left(Z^{\Gamma} \mathbf{v}\right), R^{\Gamma} \mathbf{v}\right\rangle_{\tau, \Gamma}+k\left\langle\mathbf{V}\left(R^{\Gamma} \mathbf{v}\right), Z^{\Gamma} \mathbf{v}\right\rangle_{\tau, \Gamma} .
\end{aligned}
$$

$V$ is an elliptic operator and from [13, Lemma 8] we know the ellipticity of V. For the first two terms of the right-hand side of (1.3.18), by using Lemma 8 we get

$$
\begin{aligned}
& \frac{1}{k}\left\langle V\left(\operatorname{div}_{\Gamma}\left(R^{\Gamma} \mathbf{v}\right)\right), \operatorname{div}_{\Gamma}\left(R^{\Gamma} \mathbf{v}\right)\right\rangle_{\Gamma}+k\left\langle\mathbf{V}\left(Z^{\Gamma} \mathbf{v}\right), Z^{\Gamma} \mathbf{v}\right\rangle_{\tau, \Gamma} \\
& \geq c\left(\left\|\operatorname{div}_{\Gamma}\left(R^{\Gamma} \mathbf{v}\right)\right\|_{H^{-1 / 2}(\Gamma)}^{2}+\left\|Z^{\Gamma} \mathbf{v}\right\|_{\mathbf{W}^{-1 / 2}(\Gamma)}^{2}\right) \\
& \underbrace{\geq}_{\text {Lemma } 8} c\left(\left\|R^{\Gamma} \mathbf{v}\right\|_{\mathbf{W}^{-1 / 2}(\Gamma)}^{2}+\left\|Z^{\Gamma} \mathbf{v}\right\|_{\mathbf{W}^{-1 / 2}(\Gamma)}^{2}\right) \geq c\|\mathbf{v}\|_{\mathbf{W}^{-1 / 2}(\Gamma)}^{2} .
\end{aligned}
$$


From [13, Lemma 9] we know that $\left\langle\mathbf{V}_{k} \cdot, \cdot\right\rangle_{\tau, \Gamma}$ is a compact bilinear form from $\mathbf{H}_{\times}^{1 / 2}(\Gamma) \times \mathbf{H}_{\times}^{-1 / 2}(\Gamma)$ or $\mathbf{H}_{\times}^{-1 / 2}(\Gamma) \times \mathbf{H}_{\times}^{1 / 2}(\Gamma)$ to $\mathbb{C}$. From [13, Lemma 7] and [33, Lemma 3.2], we know that $\mathbf{V}_{k}-\mathbf{V}$ is compact. We consider the left three terms of the right-hand side of (1.3.18) and know that the following bilinear form is also compact

$$
\begin{aligned}
k\left\langle\left(\mathbf{V}_{k}-\mathbf{V}\right)\left(R^{\Gamma} \mathbf{v}\right), R^{\Gamma} \mathbf{v}\right\rangle_{\tau, \Gamma} & +k\left\langle\left(\mathbf{V}_{k}-\mathbf{V}\right)\left(Z^{\Gamma} \mathbf{v}\right), R^{\Gamma} \mathbf{v}\right\rangle_{\tau, \Gamma} \\
& +k\left\langle\left(\mathbf{V}-\mathbf{V}_{k}\right)\left(R^{\Gamma} \mathbf{v}\right), Z^{\Gamma} \mathbf{v}\right\rangle_{\tau, \Gamma}
\end{aligned}
$$

Then we can define a compact bilinear form $c_{\Gamma}$ according (1.3.18) and (1.3.19). The proof is done.

If $k^{2}$ is not an eigenvalue of the eigenvalue problem of the Maxwell's equations, by [13, Theorem 4] and Fredholm's alternative, we have the existence and uniqueness of a solution $\boldsymbol{\sigma} \in \mathbf{W}^{-1 / 2}(\Gamma)$ of (1.3.17).

\subsubsection{Galerkin-BEMs}

In this section we go to the last step (1c) in Fig. 1.1 to introduce Galerkin-BEMs for (1.3.17). As in Section 1.2.5 we only consider triangular meshes. The basic definitions of meshes and boundary element spaces have been given in Section 1.2.5. In this section we define 2D triangular Raviart-Thomas elements as boundary elements.

Definition 4. A $2 D$ triangular Raviart-Thomas element of degree $l$ is defined by $\left(\mathcal{C}, \mathbf{P}_{\mathcal{C}}, \Sigma_{\mathcal{C}}\right)$

- $\mathbf{P}_{\mathcal{C}}$ is a polynomial vector space on $\Omega_{\mathcal{C}}$. Let $P_{\mathcal{C}}$ be a polynomial function space with maximum total degree $l-1$ and let $\tilde{P}_{\mathcal{C}}$ be a homogeneous polynomial function space with total degree exactly $l-1$ in two variables. $\mathbf{P}_{\mathcal{C}}$ with degree $l$ is defined as $\mathbf{P}_{\mathcal{C}}:=\left(P_{\mathcal{C}}\right)^{2} \oplus \tilde{P}_{\mathcal{C}} x$.

- The degrees of freedom $\Sigma_{\mathcal{C}}$ are defined by two parts. Let $l_{i}$ be a linear functional for $i=1,2,3$ and

$$
l_{i}(\mathbf{v}):=\int_{e_{i}} \mathbf{v}(x) \cdot \mathbf{n}_{e_{i}}(x) p(x) \mathrm{d} s_{x}
$$

for $\mathbf{v} \in \mathbf{P}_{\mathcal{C}}$ and $p \in P_{e_{i}}$ with degree $l-1$. $P_{e_{i}}$ is a polynomial function space defined on $e_{i}$ and $e_{i}$ is an edge of a cell. $\mathbf{n}_{e_{i}}$ is a tangential normal to $e_{i}$ for $i=1,2,3 . l_{4}$ is a linear functional with

$$
l_{4}(\mathbf{v}):=\int_{\Omega} \mathbf{v}(x) \cdot \mathbf{p}(x) \mathrm{d} x
$$

for $\mathbf{v} \in \mathbf{P}_{\mathcal{C}}$ and $\mathbf{p} \in\left(P_{\mathcal{C}}\right)^{2}$ with degree $l-2$.

For the lowest order Raviart-Thomas boundary element, we just need the first three degrees of freedom. A cell $\mathcal{C}$ and a boundary element $\left(\mathcal{C}, \mathbf{P}_{\mathcal{C}}, \Sigma_{\mathcal{C}}\right)$ could be defined from a reference cell by a mapping and a reference boundary element by the Piola transformation respectively. The definition of boundary element spaces is the same as in Section 1.2.5. We use the notation $\mathbf{W}_{h}^{-1 / 2}\left(\Gamma_{h}\right)$ for Raviart-Thomas finite element spaces of degree one. 
The discretization of (1.3.17) is to find $\boldsymbol{\sigma}_{h} \in \mathbf{W}_{h}^{-1 / 2}\left(\Gamma_{h}\right)$ such that

$$
\left\langle\mathbf{S}_{k}\left(\boldsymbol{\sigma}_{h}\right), \boldsymbol{\chi}_{h}\right\rangle_{\tau, \Gamma}=\left\langle\left(\frac{1}{2} I-\mathbf{C}_{k}\right) \mathbf{f}, \boldsymbol{\chi}_{h}\right\rangle_{\tau, \Gamma}
$$

for all $\boldsymbol{\chi}_{h} \in \mathbf{W}_{h}^{-1 / 2}\left(\Gamma_{h}\right)$. Let $\left\{\boldsymbol{\Phi}_{i}\right\}_{i=1}^{N}$ be a basis of $\mathbf{W}_{h}^{-1 / 2}\left(\Gamma_{h}\right)$. We use $\boldsymbol{\sigma}_{h}=\sum_{i=1}^{N} \xi_{i} \boldsymbol{\Phi}_{i}$ in (1.3.20) and get a linear system

$$
A \xi=b .
$$

The elements in $A$ and $b$ are calculated by

$$
\begin{gathered}
A[i, j]=-k \int_{\Gamma} \int_{\Gamma \backslash\{x\}} \boldsymbol{\Phi}_{j}(y) \cdot \boldsymbol{\Phi}_{i}(x) E_{k}(x, y) \mathrm{d} s_{y} \mathrm{~d} s_{x} \\
+\frac{1}{k} \int_{\Gamma} \int_{\Gamma \backslash\{x\}} \operatorname{div}_{\Gamma}\left(\boldsymbol{\Phi}_{j}\right)(y) \operatorname{div}_{\Gamma}\left(\boldsymbol{\Phi}_{i}\right)(x) E_{k}(x, y) \mathrm{d} s_{y} \mathrm{~d} s_{x}, \\
b[i]=\frac{1}{2} \int_{\Gamma} \mathbf{f}(x) \times \mathbf{n}(x) \cdot \boldsymbol{\Phi}_{i}(x) \mathrm{d} s_{x}+\int_{\Gamma} \int_{\Gamma \backslash\{x\}} \nabla_{x} E_{k}(x, y) \cdot\left(\mathbf{f}(y) \times \boldsymbol{\Phi}_{i}(x)\right) \mathrm{d} s_{y} \mathrm{~d} s_{x} .
\end{gathered}
$$

Now we begin to study the error estimates for (1.3.20). As in Section 1.2.5 we also prove the best approximation, the optimal convergence and a super convergent result.

Lemma 10. There exists $h_{0}>0$ such that for all $\chi_{h} \in \mathbf{W}_{h}^{-1 / 2}\left(\Gamma_{h}\right)$ and $0<h<h_{0}$ we have

$$
c_{s}\left\|\boldsymbol{\chi}_{h}\right\|_{\mathbf{W}^{-1 / 2}(\Gamma)} \leq \sup _{\boldsymbol{\chi}_{h}^{\prime} \in \mathbf{W}_{h}^{-1 / 2}\left(\Gamma_{h}\right),\left\|\boldsymbol{\chi}_{h}^{\prime}\right\|_{\mathbf{W}^{-1 / 2}(\Gamma)}>0} \frac{\left\langle\mathbf{S}_{k} \boldsymbol{\chi}_{h}, \boldsymbol{\chi}_{h}^{\prime}\right\rangle_{\Gamma}}{\left\|\boldsymbol{\chi}_{h}^{\prime}\right\|_{\mathbf{W}^{-1 / 2}(\Gamma)}} .
$$

Proof. The basic ideas of this proof is given in [13, Section 9.1]. In the proof we need to use the generalized Gårding inequality. If we compare Lemma 3 with Lemma 9, we find that we use a compact bilinear form $c_{\Gamma}$ in Lemma 9 and a compact operator $C_{V}$ in Lemma 3. So we define an operator $\mathbf{T}: \mathbf{W}^{-1 / 2}(\Gamma) \rightarrow \mathbf{W}^{-1 / 2}(\Gamma)$ corresponding to $c_{\Gamma}$ by

$$
\left\langle\mathbf{S}_{k} \boldsymbol{\chi}, \mathbf{T} \widehat{\chi}\right\rangle_{\tau, \Gamma}=c_{\Gamma}(\boldsymbol{\chi}, \widehat{\chi}) \quad \forall \boldsymbol{\chi} \in \mathbf{W}^{-1 / 2}(\Gamma) .
$$

$\mathbf{S}_{k}$ defines an isomorphism as in the discussion in Section 1.3.4. Since $c_{\Gamma}$ is a compact bilinear form, $\mathbf{T}$ is a compact operator by the inf-sup condition for $\mathbf{S}_{k}$.

We begin to consider the right-hand side of (1.3.21).

$$
\begin{aligned}
& \left\langle\mathbf{S}_{k} \boldsymbol{\chi}_{h},\left(\mathbf{P}_{h}^{X} X^{\Gamma}+\mathbf{P}_{h}^{T} \mathbf{T}\right) \boldsymbol{\chi}_{h}\right\rangle_{\tau, \Gamma} \\
= & \left\langle\mathbf{S}_{k} \boldsymbol{\chi}_{h},\left(X^{\Gamma}+\mathbf{T}\right) \boldsymbol{\chi}_{h}\right\rangle_{\tau, \Gamma}-\left\langle\mathbf{S}_{k} \boldsymbol{\chi}_{h},\left(\left(I-\mathbf{P}_{h}^{X}\right) X^{\Gamma}+\left(I-\mathbf{P}_{h}^{T}\right) \mathbf{T}\right) \boldsymbol{\chi}_{h}\right\rangle_{\tau, \Gamma},
\end{aligned}
$$

where $\mathbf{P}_{h}^{X}$ and $\mathbf{P}_{h}^{T}$ are two continuous projection operators to $\mathbf{W}_{h}^{-1 / 2}\left(\Gamma_{h}\right)$. The definitions of $\mathbf{P}_{h}^{X}$ and $\mathbf{P}_{h}^{T}$ and their uniform convergence could be found in [13, Section 9.1]. We get

$$
\begin{array}{r}
\left|\left\langle\mathbf{S}_{k} \boldsymbol{\chi}_{h},\left(\mathbf{P}_{h}^{X} X^{\Gamma}+\mathbf{P}_{h}^{T} \mathbf{T}\right) \chi_{h}\right\rangle_{\tau, \Gamma}\right|+\left|\left\langle\mathbf{S}_{k} \boldsymbol{\chi}_{h},\left(\left(I-\mathbf{P}_{h}^{X}\right) X^{\Gamma}+\left(I-\mathbf{P}_{h}^{T}\right) \mathbf{T}\right) \chi_{h}\right\rangle_{\tau, \Gamma}\right| \\
\geq\left|\left\langle\mathbf{S}_{k} \boldsymbol{\chi}_{h},\left(X^{\Gamma}+\mathbf{T}\right) \boldsymbol{\chi}_{h}\right\rangle_{\tau, \Gamma}\right|
\end{array}
$$


Now we can use the generalized Gårding inequality and get

$$
\begin{gathered}
\left|\left\langle\mathbf{S}_{k} \boldsymbol{\chi}_{h},\left(X^{\Gamma}+\mathbf{T}\right) \boldsymbol{\chi}_{h}\right\rangle_{\tau, \Gamma}\right|=\left|\left\langle\mathbf{S}_{k} \boldsymbol{\chi}_{h}, X^{\Gamma} \boldsymbol{\chi}_{h}\right\rangle_{\tau, \Gamma}+c_{\Gamma}\left(\boldsymbol{\chi}_{h}, \boldsymbol{\chi}_{h}\right)\right| \geq c_{g}\left\|\boldsymbol{\chi}_{h}\right\|_{\mathbf{W}^{-1 / 2}(\Gamma)}^{2} \\
\left|\left\langle\mathbf{S}_{k} \boldsymbol{\chi}_{h},\left(\left(I-\mathbf{P}_{h}^{X}\right) X^{\Gamma}+\left(I-\mathbf{P}_{h}^{T}\right) \mathbf{T}\right) \boldsymbol{\chi}_{h}\right\rangle_{\tau, \Gamma}\right| \\
\leq c\left(\left\|\left(I-\mathbf{P}_{h}^{X}\right) X^{\Gamma}\right\|+\left\|\left(I-\mathbf{P}_{h}^{T}\right) \mathbf{T}\right\|\right)\left\|\boldsymbol{\chi}_{h}\right\|_{\mathbf{W}^{-1 / 2}(\Gamma)}^{2}
\end{gathered}
$$

By using the uniform convergence of $\mathbf{P}_{h}^{X}$ and $\mathbf{P}_{h}^{T}$, we could find a $h_{0}$ such that

$$
\left|\left\langle\mathbf{S}_{k} \boldsymbol{\chi}_{h},\left(\left(I-\mathbf{P}_{h}^{X}\right) X^{\Gamma}+\left(I-\mathbf{P}_{h}^{T}\right) \mathbf{T}\right) \boldsymbol{\chi}_{h}\right\rangle_{\tau, \Gamma}\right| \leq c\left\|\boldsymbol{\chi}_{h}\right\|_{\mathbf{W}^{-1 / 2}(\Gamma)}^{2}
$$

for $h<h_{0}$ and $c<c_{g}$. Then we have

$$
\left|\left\langle\mathbf{S}_{k} \boldsymbol{\chi}_{h},\left(\mathbf{P}_{h}^{X} X^{\Gamma}+\mathbf{P}_{h}^{T} \mathbf{T}\right) \boldsymbol{\chi}_{h}\right\rangle_{\tau, \Gamma}\right| \geq\left(c_{g}-c\right)\left\|\boldsymbol{\chi}_{h}\right\|_{\mathbf{W}^{-1 / 2}(\Gamma)}^{2} .
$$

By the continuity of the operators, the proof is done.

Lemma 11. There exists $c>0$ such that for the solutions of (1.3.17) and (1.3.20) we have

$$
\left\|\boldsymbol{\sigma}-\boldsymbol{\sigma}_{h}\right\|_{\mathbf{W}^{-1 / 2}(\Gamma)} \leq c \inf _{\chi_{h} \in \mathbf{W}_{h}^{-1 / 2}\left(\Gamma_{h}\right)}\left\|\boldsymbol{\sigma}-\chi_{h}\right\|_{\mathbf{W}^{-1 / 2}(\Gamma)}
$$

Proof. From (1.3.17) and (1.3.20) we have

$$
\left\langle\mathbf{S}_{k} \boldsymbol{\sigma}_{h}, \boldsymbol{\chi}_{h}\right\rangle_{\tau, \Gamma}=\left\langle\mathbf{S}_{k} \boldsymbol{\sigma}, \boldsymbol{\chi}_{h}\right\rangle_{\tau, \Gamma} \quad \text { for all } \boldsymbol{\chi}_{h} \in \mathbf{W}_{h}^{-1 / 2}\left(\Gamma_{h}\right)
$$

This defines a Galerkin projection $G_{h \mathbf{S}_{k}} \boldsymbol{\sigma}=\boldsymbol{\sigma}_{h}$. By the stability from the LBB condition $G_{h \mathbf{S}_{k}}$ is bounded. Then we have

$$
\begin{aligned}
\left\|\boldsymbol{\sigma}-\boldsymbol{\sigma}_{h}\right\|_{\mathbf{W}^{-1 / 2}(\Gamma)} & =\left\|\boldsymbol{\sigma}-G_{h \mathbf{S}_{k}} \chi_{h}+G_{h \mathbf{S}_{k}} \chi_{h}-\boldsymbol{\sigma}_{h}\right\|_{\mathbf{W}^{-1 / 2}(\Gamma)} \\
& \leq\left\|\boldsymbol{\sigma}-\chi_{h}\right\|_{\mathbf{W}^{-1 / 2}(\Gamma)}+\left\|G_{h \mathbf{S}_{k}}\left(\chi_{h}-\boldsymbol{\sigma}\right)\right\|_{\mathbf{W}^{-1 / 2}(\Gamma)} \\
& \leq(1+c)\left\|\boldsymbol{\sigma}-\boldsymbol{\chi}_{h}\right\|_{\mathbf{W}^{-1 / 2}(\Gamma)} .
\end{aligned}
$$

The proof is done.

The approximation property of $\mathbf{W}_{h}^{-1 / 2}\left(\Gamma_{h}\right)$ for $\chi \in \mathbf{H}_{\times}^{s}\left(\operatorname{div}_{\Gamma}, \Gamma\right)$ is given in [13, Theorem 14]

$$
\left\|\chi-\mathbf{Q}_{h} \boldsymbol{\chi}\right\|_{\mathbf{W}^{-1 / 2}(\Gamma)} \leq c h^{s+1 / 2}\|\chi\|_{\mathbf{H}_{\times}^{s}\left(\operatorname{div}_{\Gamma}, \Gamma\right)} \quad \text { for } s \in[-1 / 2, l],
$$

where $\mathrm{Q}_{h} \chi$ is an orthogonal projection of $\chi$ to $\mathbf{W}_{h}^{-1 / 2}\left(\Gamma_{h}\right)$ with respect to the inner product of $\mathbf{W}^{-1 / 2}(\Gamma)$ and $l$ is the order of Raviart-Thomas boundary element spaces. We use (1.3.23) in (1.3.22) and get a quasi-optimal error estimate

$$
\left\|\boldsymbol{\sigma}-\boldsymbol{\sigma}_{h}\right\|_{\mathbf{W}^{-1 / 2}(\Gamma)} \leq c h^{s+1 / 2}\|\boldsymbol{\sigma}\|_{\mathbf{H}_{\times}^{s}\left(\operatorname{div}_{\Gamma}, \Gamma\right)} .
$$

As for the Helmholtz equation we need to calculate errors in the $\mathbf{L}_{\mathbf{t}}^{2}(\Gamma)$-norm and we 
just consider the lowest order Raviart-Thomas boundary element with $l=1$.

Theorem 4. Let $\boldsymbol{\sigma} \in \mathbf{H}_{\times}^{s}\left(\operatorname{div}_{\Gamma}, \Gamma\right)$ for $s \in[-1 / 2,1]$. We have

$$
\left\|\boldsymbol{\sigma}-\boldsymbol{\sigma}_{h}\right\|_{\mathbf{L}_{\mathbf{t}}^{2}(\Gamma)} \leq c h^{s}\|\boldsymbol{\sigma}\|_{\mathbf{H}_{\times}^{s}\left(\operatorname{div}_{\Gamma}, \Gamma\right)} .
$$

Proof. The proof is similar with Theorem 2 . The inverse inequality could be found in [17, Lemma 10]. We find

$$
\begin{aligned}
&\left\|\boldsymbol{\sigma}-\boldsymbol{\sigma}_{h}\right\|_{\mathbf{L}_{\mathbf{t}}^{2}(\Gamma)} \leq\left\|\boldsymbol{\sigma}-\mathbf{Q}_{h} \boldsymbol{\sigma}\right\|_{\mathbf{L}_{\mathbf{t}}^{2}(\Gamma)}+\left\|\mathbf{Q}_{h} \boldsymbol{\sigma}-\boldsymbol{\sigma}_{h}\right\|_{\mathbf{L}_{\mathbf{t}}^{2}(\Gamma)} \\
& \underbrace{\leq}_{\text {inverse inequality }}\left\|\boldsymbol{\sigma}-\mathbf{Q}_{h} \boldsymbol{\sigma}\right\|_{\mathbf{L}_{\mathbf{t}}^{2}(\Gamma)}+c h^{-1 / 2}\left\|\mathbf{Q}_{h} \boldsymbol{\sigma}-\boldsymbol{\sigma}_{h}\right\|_{\mathbf{W}^{-1 / 2}(\Gamma)} \\
& \quad \leq\left\|\boldsymbol{\sigma}-\mathbf{Q}_{h} \boldsymbol{\sigma}\right\|_{\mathbf{L}_{\mathbf{t}}^{2}(\Gamma)}+c h^{-1 / 2}\left(\left\|\mathbf{Q}_{h} \boldsymbol{\sigma}-\boldsymbol{\sigma}\right\|_{\mathbf{W}^{-1 / 2}(\Gamma)}+\left\|\boldsymbol{\sigma}-\boldsymbol{\sigma}_{h}\right\|_{\mathbf{W}^{-1 / 2}(\Gamma)}\right) .
\end{aligned}
$$

By (1.3.23) and (1.3.24), the proof is done.

\subsubsection{Numerical tests}

We consider one example on the boundary of a unit cube $(0,1)^{3}$. A analytical solution used for the test is

$$
\mathbf{u}(x)=\left(\begin{array}{c}
x_{2} \cos \left(x_{3}\right)+x_{3} \cos \left(x_{2}\right) \\
x_{1} \cos \left(x_{3}\right)+x_{3} \cos \left(x_{1}\right) \\
x_{1} \cos \left(x_{2}\right)+x_{2} \cos \left(x_{1}\right)
\end{array}\right)
$$

In Table 1.3 the first column is the level of meshes, the second column is the number of degrees of freedom, the third and fifth columns are $\mathbf{L}^{2}$-error for Dirichlet and Neumann boundary value problems respectively, and the fourth and sixth columns are the rate of convergence $(s)$ in $O\left(h^{s}\right)$. Fig.1.4a is the result of the Dirichlet boundary value problem of (1.3.25). Fig.1.4b is the result of the Neumann boundary value problem of (1.3.25). "CR" means the rate of convergence $(s)$ and $\boldsymbol{\sigma}_{h}^{n}$ is the approximated solution calculated by using a mesh of level $n$. The formulae are

$$
\begin{gathered}
\left\|\boldsymbol{\sigma}_{h}-\boldsymbol{\sigma}\right\|_{\mathbf{L}^{2}(\Gamma)}^{2}=\int_{\Gamma}\left(\boldsymbol{\sigma}_{h}(x)-\boldsymbol{\sigma}(x)\right) \cdot\left(\boldsymbol{\sigma}_{h}(x)-\boldsymbol{\sigma}(x)\right) \mathrm{d} s_{x}, \\
\mathrm{CR}=\log _{2} \frac{\left\|\boldsymbol{\sigma}_{h}^{n-1}-\boldsymbol{\sigma}\right\|_{\mathbf{L}^{2}(\Gamma)}}{\left\|\boldsymbol{\sigma}_{h}^{n}-\boldsymbol{\sigma}\right\|_{\mathbf{L}^{2}(\Gamma)}}
\end{gathered}
$$

\begin{tabular}{|c|c|c|c|c|c|}
\hline level & dof & Error $_{D}$ & $\mathrm{CR}_{D}$ & Error $_{N}$ & $\mathrm{CR}_{N}$ \\
\hline 0 & 36 & 0.52560 & - & 0.69761 & - \\
\hline 1 & 144 & 0.26010 & 1.0149 & 0.27707 & 1.3322 \\
\hline 2 & 576 & 0.13016 & 0.9987 & 0.13268 & 1.0623 \\
\hline 3 & 2304 & 0.06476 & 1.0072 & 0.06488 & 1.0320 \\
\hline
\end{tabular}

Table 1.3 Accuracy of Galerkin-BEMs for Dirichlet and Neumann boundary value problems for Maxwell's equations 


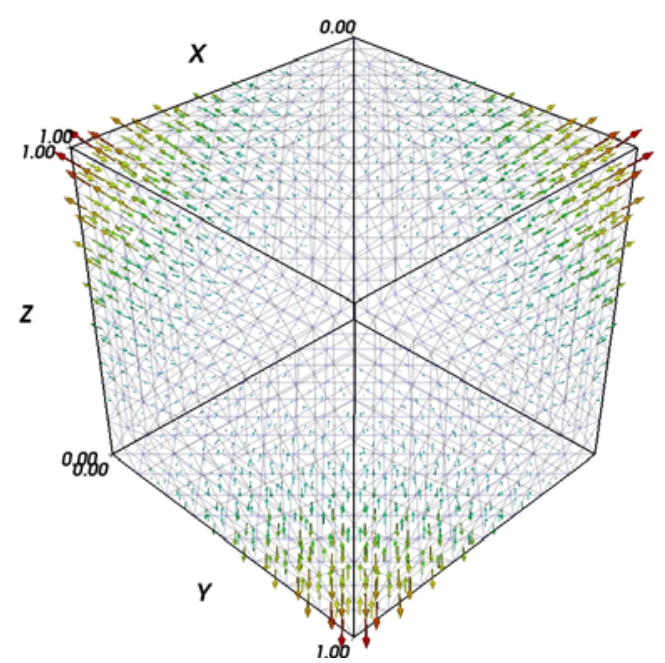

(a) Dirichlet boundary value problem

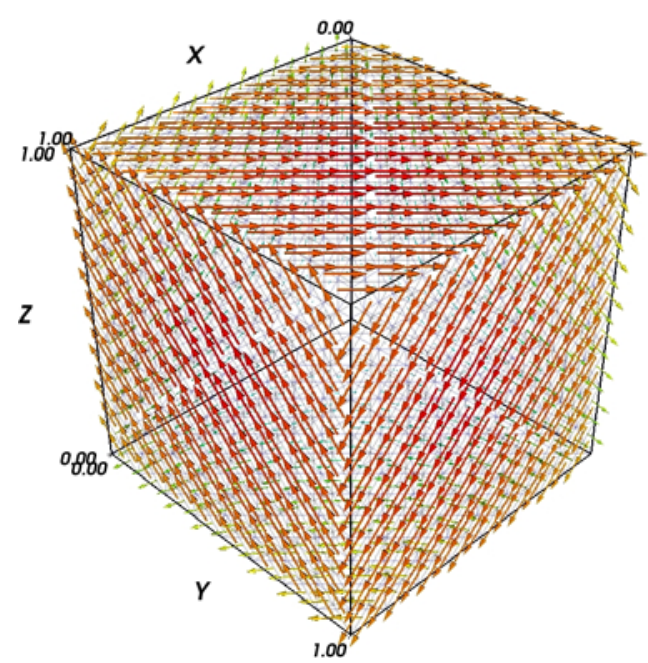

(b) Neumann boundary value problem

Figure 1.4 Dirichlet and Neumann boundary value problems for Maxwell's equations

In the fourth and sixth columns of Table 1.3 we observe a linear convergence for the lowest order Raviat-Thomas boundary element space. The perturbation in the rate of convergence is from the approximation of the linear form on the right-hand side of the variational formulation and numerical integrations. 


\section{Chapter 2}

\section{Domain Decomposition Methods}

Domain decomposition methods (DDM) can be used for the coupling of different physical problems in different domains, the coupling of different numerical methods in different domains, parallel computing and preconditioners for iterative methods. The basic idea of domain decomposition methods for boundary value problems is to reduce the solution of the boundary value problem in the whole domain to the solution of problems of the same type in subdomains by using interface conditions [56, 57, 64, 63, 68]. For a boundary integral equation, the solution on the boundary of the whole domain is reduced to the solution on the boundaries of subdomains. The calculation of band strucutres of photonic crystals is an inhomogeneous problem for the propagation of electromagnetic waves in an inhomogeneous dielectric medium. In our case we assume that the medium has different permittivity and permeability in different subdomains and in each subdomain the permittivity and permeability are constant. This is the coupling of different physical problems in different subdomains and we need to use DDMs to deal with this problem. Domain decomposition methods can also be used for the coupling of BEMs and FEMs. This is a very good combination of the advantages of BEMs and FEMs and it is very suitable for solving the transmission problem for electromagnetic waves.

\subsection{The Helmholtz case}

\subsubsection{Interface problem}

In this section we consider an inhomogeneous problem for the Helmholtz equation in $\Omega:=\Omega^{e x} \cup \overline{\Omega^{i n}}$ and the coefficient $\alpha$ in the equation has different values in $\Omega^{e x}$ and $\Omega^{i n}$. Since we use interface conditions for the coupling in this problem, we call it the interface problem. The interface problem with the Dirichlet boundary condition is

$$
\begin{aligned}
-\nabla \cdot\left(\frac{1}{\alpha} \nabla u(x)\right)-k^{2} u(x) & =0 \\
u(x) & =f(x) \quad x \in \Omega^{e x} \bigcup \Omega^{i n}, \\
x & \in \Gamma:=\partial \Omega,
\end{aligned}
$$

where $k$ is a positive real number, $\alpha=\alpha^{e x}$ in $\Omega^{e x}, \alpha=\alpha^{i n}$ in $\Omega^{i n}$, and $f$ is the given data. $\alpha^{e x}$ and $\alpha^{i n}$ are positive constants. 
We have two interface conditions for this problem

$$
\begin{aligned}
\gamma_{0}^{e x}\left(u^{e x}\right)(x) & =\gamma_{0}^{i n}\left(u^{i n}\right)(x) & & \forall x \in \Gamma^{I}, \\
\alpha^{e x} \gamma_{1}^{e x}\left(u^{e x}\right)(x) & =-\alpha^{i n} \gamma_{1}^{i n}\left(u^{i n}\right)(x) & & \forall x \in \Gamma^{I} .
\end{aligned}
$$

where $u^{e x}:=\left.u\right|_{\Omega^{e x}}, u^{i n}:=\left.u\right|_{\overline{\Omega^{i n}}}, \gamma_{0}^{e x}, \gamma_{0}^{i n}, \gamma_{1}^{e x}$, and $\gamma_{1}^{i n}$ are Dirichlet and Neumann trace operators from $\Omega^{e x}$ and $\Omega^{i n}$, respectively, and $\Gamma^{I}:=\Gamma^{e x} \bigcap \Gamma^{i n}$ with $\Gamma^{e x}:=\partial \Omega^{e x}$ and $\Gamma^{i n}:=\partial \Omega^{i n}$. The definitions of the trace operators could be found in Section 1.2.3.

\subsubsection{Domain decomposition method}

We define $\sigma^{e x}:=\gamma_{1}^{e x}\left(u^{e x}\right), \sigma^{i n}:=\gamma_{1}^{i n}\left(u^{i n}\right), \varphi^{e x}:=\gamma_{0}^{e x}\left(u^{e x}\right)$ and $\varphi^{i n}:=\gamma_{0}^{i n}\left(u^{i n}\right)$. By using (1.2.11) we may define two Calderon projections for $\left(\varphi^{e x}, \sigma^{e x}\right)$ and $\left(\varphi^{i n}, \sigma^{i n}\right)$ respectively as

$$
\begin{aligned}
& \left(\begin{array}{c}
\varphi^{e x} \\
\sigma^{e x}
\end{array}\right)=\left(\begin{array}{cc}
\frac{1}{2} I-K_{k^{\Gamma^{e x}}}^{\Gamma^{e x}} & V_{k^{e x}}^{\Gamma^{e x}} \\
D_{k^{e x}}^{\Gamma^{e x}} & \frac{1}{2} I+K^{\prime{ }^{e x}}
\end{array}\right)\left(\begin{array}{c}
\varphi^{e x} \\
\sigma^{e x}
\end{array}\right), \\
& \left(\begin{array}{c}
\varphi^{i n} \\
\sigma^{i n}
\end{array}\right)=\left(\begin{array}{cc}
\frac{1}{2} I-K_{k^{i n}}^{\Gamma^{i n}} & V_{k^{i n}}^{\Gamma^{i n}} \\
D_{k^{i n}}^{\Gamma^{i n}} & \frac{1}{2} I+K^{\prime \Gamma^{i n}}
\end{array}\right)\left(\begin{array}{c}
\varphi^{i n} \\
\sigma^{i n}
\end{array}\right) .
\end{aligned}
$$

where $k^{e x}=\sqrt{\alpha^{e x}} k, k^{i n}=\sqrt{\alpha^{i n}} k$, and the definitions of the boundary integral operators are given in Section 1.2.3 on $\Gamma^{e x}$ and $\Gamma^{i n}$ respectively.

We use the second equations of (2.1.3a) and (2.1.3b) in (2.1.2b) and get

$$
\begin{aligned}
\alpha^{e x}\left(D_{k^{e x}}^{\Gamma^{e x}} \varphi^{e x}+\right. & \left.\left(\frac{1}{2} I+{K^{\prime}}_{k^{e x}}^{\Gamma^{e x}}\right) \sigma^{e x}\right) \\
& +\alpha^{i n}\left(D_{k^{i n}}^{\Gamma^{i n}} \varphi^{i n}+\left(\frac{1}{2} I+{K^{\prime}}_{k^{i n}}^{\Gamma^{i n}}\right) \sigma^{i n}\right)=0 .
\end{aligned}
$$

From (2.1.2a) and (2.1.1b), we have $\varphi^{e x}=\varphi^{i n}$ on $\Gamma^{I}$ and $\varphi^{e x}=f$ on $\Gamma$. We define $\varphi^{I}$ as $\varphi^{I}:=\varphi^{e x}=\varphi^{i n}$ on $\Gamma^{I}$ and $\varphi^{I}=0$ on $\Gamma$. We also assume that the extension of $f$ on $\Gamma^{I}$ is zero. We assume that the exterior unit normal on $\Gamma^{I}$ and $\Gamma$ is the same as on $\Gamma^{e x}$. We use $\varphi^{I}$ and $f$ in (2.1.4) and get

$$
\begin{aligned}
& \left(\alpha^{e x} D_{k^{e x}}^{\Gamma^{I}}+\alpha^{i n} D_{k^{i n}}^{\Gamma^{I}}\right)\left(\varphi^{I}\right)(x)+\alpha^{e x}\left(\frac{1}{2} I+{K^{\prime}}_{k^{e x}}^{\Gamma^{e x}}\right)\left(\sigma^{e x}\right)(x) \\
& +\alpha^{i n}\left(\frac{1}{2} I+{K^{\prime}}_{k^{i n}}^{\Gamma^{i n}}\right)\left(\sigma^{i n}\right)(x)=-\alpha^{e x} D_{k^{e x}}^{\Gamma}(f)(x)
\end{aligned} \quad \forall x \in \Gamma^{I} .
$$

From the first equations in (2.1.3a) and (2.1.3b) we get

$$
\begin{aligned}
\left(\frac{1}{2} I+K_{k^{e x}}^{\Gamma^{I}}\right)\left(\varphi^{I}\right)(x) & -V_{k^{e x}}^{\Gamma^{e x}}\left(\sigma^{e x}\right)(x) \\
& =-\left(\frac{1}{2} I+K_{k^{e x}}^{\Gamma}\right)(f)(x) \quad \forall x \in \Gamma^{e x},
\end{aligned}
$$




$$
\left(\frac{1}{2} I+K_{k^{i n}}^{\Gamma^{i n}}\right)\left(\varphi^{I}\right)(x)-V_{k^{i n}}^{\Gamma^{i n}}\left(\sigma^{i n}\right)(x)=0 \quad \forall x \in \Gamma^{i n} .
$$

The combination of (2.1.5), (2.1.6) and (2.1.7) is the system of equations for the solution on the boundaries of subdomains.

\subsubsection{Variational formulation}

By using the left hand sides of (2.1.5), (2.1.6) and (2.1.7), we define three operators

$$
\begin{aligned}
& \mathbf{F}_{1}: H^{1 / 2}\left(\Gamma^{I}\right) \times H^{-1 / 2}\left(\Gamma^{e x}\right) \times H^{-1 / 2}\left(\Gamma^{i n}\right) \rightarrow H^{-1 / 2}\left(\Gamma^{I}\right), \\
& \mathbf{F}_{2}: H^{1 / 2}\left(\Gamma^{I}\right) \times H^{-1 / 2}\left(\Gamma^{e x}\right) \rightarrow H^{1 / 2}\left(\Gamma^{e x}\right), \\
& \mathbf{F}_{3}: H^{1 / 2}\left(\Gamma^{I}\right) \times H^{-1 / 2}\left(\Gamma^{i n}\right) \rightarrow H^{1 / 2}\left(\Gamma^{i n}\right),
\end{aligned}
$$

which result into the following variational problem.

Find $\left(\varphi^{I}, \sigma^{e x}, \sigma^{i n}\right) \in H^{1 / 2}\left(\Gamma^{I}\right) \times H^{-1 / 2}\left(\Gamma^{e x}\right) \times H^{-1 / 2}\left(\Gamma^{i n}\right)$ such that

$$
\begin{aligned}
& \left\langle\mathbf{F}_{1}\left(\varphi^{I}, \sigma^{e x}, \sigma^{i n}\right), v^{I}\right\rangle_{\Gamma^{I}}=\left\langle-\alpha^{e x} D_{k^{e x}}^{\Gamma}(f), v^{I}\right\rangle_{\Gamma^{I}} \\
& \left\langle\mathbf{F}_{2}\left(\varphi^{I}, \sigma^{e x}\right), \chi^{e x}\right\rangle_{\Gamma^{e x}}=\left\langle-\left(\frac{1}{2} I+K_{k^{e x}}^{\Gamma}\right)(f), \chi^{e x}\right\rangle_{\Gamma^{e x}}, \\
& \left\langle\mathbf{F}_{3}\left(\varphi^{I}, \sigma^{i n}\right), \chi^{i n}\right\rangle_{\Gamma^{i n}}=0
\end{aligned}
$$

for all $\left(v^{I}, \chi^{e x}, \chi^{i n}\right) \in H^{1 / 2}\left(\Gamma^{I}\right) \times H^{-1 / 2}\left(\Gamma^{e x}\right) \times H^{-1 / 2}\left(\Gamma^{i n}\right)$.

Theorem 5. If $k^{e x}$ and $k^{\text {in }}$ are no eigenvalues of the Laplace eigenvalue problem in $\Omega^{e x}$ and $\Omega^{\text {in }}$, respectively, then there exists an unique solution for (2.1.8).

Proof. A similar proof is given in [58, Section 1.1.8]. We recall that $\varphi^{I}=0$ on $\Gamma$. We can extend the operator $K_{k^{e x}}^{\Gamma^{I}}$ in (2.1.6) to $K_{k^{e x}}^{\Gamma^{e x}}$ and get

$$
\left(\frac{1}{2} I+K_{k^{e x}}^{\Gamma^{e x}}\right)\left(\varphi^{I}\right)-V_{k^{e x}}^{\Gamma^{e x}}\left(\sigma^{e x}\right)=-\left(\frac{1}{2} I+K_{k^{e x}}^{\Gamma}\right)(f) .
$$

If we consider the variational formulation

$$
\left\langle V_{k^{e x}}^{\Gamma^{e x}}\left(\sigma^{e x}\right), \chi^{e x}\right\rangle_{\Gamma^{e x}}=\left\langle\left(\frac{1}{2} I+K_{k^{e x}}^{\Gamma^{e x}}\right)\left(\varphi^{I}\right)+\left(\frac{1}{2} I+K_{k^{e x}}^{\Gamma}\right)(f), \chi^{e x}\right\rangle_{\Gamma^{e x}},
$$

and we assume that $k^{e x}$ is not an eigenvalue of the Laplace operator in $\Omega^{e x}$, from Section 1.2.4 we know the existence and uniqueness of the solution of this variational formulation. Then we have

$$
\sigma^{e x}=\left(V_{k^{e x}}^{\Gamma^{e x}}\right)^{-1}\left(\left(\frac{1}{2} I+K_{k^{e x}}^{\Gamma^{e x}}\right)\left(\varphi^{I}\right)+\left(\frac{1}{2} I+K_{k^{e x}}^{\Gamma}\right)(f)\right) .
$$

It is the same for (2.1.7) and we have

$$
\sigma^{i n}=\left(V_{k^{i n}}^{\Gamma^{i n}}\right)^{-1}\left(\frac{1}{2} I+K_{k^{i n}}^{\Gamma^{i n}}\right)\left(\varphi^{I}\right) .
$$


We can extend the operator $D_{k^{e x}}^{\Gamma^{I}}$ in (2.1.5) to $D_{k^{e x}}^{\Gamma^{e x}}$. By using the above two formulae for $\sigma^{e x}$ and $\sigma^{i n}$ in (2.1.5), we get

$$
\begin{gathered}
\alpha^{e x} \underbrace{\left[D_{k^{e x}}^{\Gamma^{e x}}+\left(\frac{1}{2} I+K^{\prime \Gamma^{e x}}\right)\left(V_{k^{e x}}^{\Gamma^{e x}}\right)^{-1}\left(\frac{1}{2} I+K_{k^{e x}}^{\Gamma^{e x}}\right)\right]}_{S_{k}^{\Gamma^{e x}}}\left(\varphi^{I}\right) \\
+\alpha^{i n} \underbrace{\left[D_{k^{i n}}^{\Gamma^{i n}}+\left(\frac{1}{2} I+K^{\prime \Gamma^{i n}}\right)\left(V_{k^{i n}}^{\Gamma^{i n}}\right)^{-1}\left(\frac{1}{2} I+K_{k^{i n}}^{\Gamma^{i n}}\right)\right]}_{S_{k}^{\Gamma^{i n}}}\left(\varphi^{I}\right) \\
=-\alpha^{e x} D_{k^{e x}}^{\Gamma^{e x}}(f)-\alpha^{e x}\left(\frac{1}{2} I+K_{k^{\prime \Gamma^{e x}}}^{\Gamma^{e x}}\right)\left(V_{k^{e x}}^{\Gamma^{e x}}\right)^{-1}\left(\frac{1}{2} I+K_{k^{e x}}^{\Gamma}\right)(f) .
\end{gathered}
$$

From the left hand side of (2.1.9), we can define two Steklov-Poincaré operators, $S_{k^{e x}}^{\Gamma^{e x}}$ and $S_{k^{i n}}^{\Gamma^{i n}}$, and from Lemma 2.3 in [53] we know that they are coercive. Since we assume that $k^{e x}$ and $k^{i n}$ are no eigenvalues of the Laplace operator in $\Omega^{e x}$ and $\Omega^{i n}$, respectively, $S_{k^{e x}}^{\Gamma^{e x}}$ and $S_{k^{i n}}^{\Gamma^{i n}}$ are injective. Then we have the existence and uniqueness of $\varphi^{I}$ in (2.1.9) by the Fredholm's alternative. By the existence and uniqueness of $\varphi^{I}$, we get the same properties for $\sigma^{e x}$ and $\sigma^{i n}$. The proof is done.

\subsubsection{Galerkin-BEM}

The Galerkin discretization of (2.1.8) is to find $\left(\varphi_{h}^{I}, \sigma_{h}^{e x}, \sigma_{h}^{i n}\right) \in S_{h}^{1}\left(\Gamma_{h}^{I}\right) \times S_{h}^{0}\left(\Gamma_{h}^{e x}\right) \times$ $S_{h}^{0}\left(\Gamma_{h}^{i n}\right)$ such that

$$
\begin{aligned}
& \left\langle\mathbf{F}_{1}\left(\varphi_{h}^{I}, \sigma_{h}^{e x}, \sigma_{h}^{i n}\right), v_{h}^{I}\right\rangle_{\Gamma^{I}}=\left\langle-\alpha^{e x} D_{k^{e x}}^{\Gamma}(f), v_{h}^{I}\right\rangle_{\Gamma^{I}}, \\
& \left\langle\mathbf{F}_{2}\left(\varphi_{h}^{I}, \sigma_{h}^{e x}\right), \chi_{h}^{e x}\right\rangle_{\Gamma^{e x}}=\left\langle-\left(\frac{1}{2} I+K_{k^{e x}}^{\Gamma}\right)(f), \chi_{h}^{e x}\right\rangle_{\Gamma^{e x}}, \\
& \left\langle\mathbf{F}_{3}\left(\varphi_{h}^{I}, \sigma_{h}^{i n}\right), \chi_{h}^{i n}\right\rangle_{\Gamma^{i n}}=0
\end{aligned}
$$

for all $\left(v_{h}^{I}, \chi_{h}^{e x}, \chi_{h}^{i n}\right) \in S_{h}^{1}\left(\Gamma_{h}^{I}\right) \times S_{h}^{0}\left(\Gamma_{h}^{e x}\right) \times S_{h}^{0}\left(\Gamma_{h}^{i n}\right) . S_{h}^{1}\left(\Gamma_{h}^{I}\right)$ is a piecewise linear function space, and $S_{h}^{0}\left(\Gamma_{h}^{e x}\right)$ and $S_{h}^{0}\left(\Gamma_{h}^{i n}\right)$ are piecewise constant function spaces.

Let $\left\{\Phi_{i}^{I}\right\}_{i=1}^{N^{I}},\left\{\Phi_{i}^{e x}\right\}_{i=1}^{N^{e x}}$ and $\left\{\Phi_{i}^{i n}\right\}_{i=1}^{N^{i n}}$ be the basis of $S_{h}^{1}\left(\Gamma_{h}^{I}\right), S_{h}^{0}\left(\Gamma_{h}^{e x}\right)$ and $S_{h}^{0}\left(\Gamma_{h}^{i n}\right)$ respectively. We use $\varphi_{h}^{I}=\sum_{i=1}^{N^{I}} \xi_{i}^{I} \Phi_{i}^{I}, \sigma_{h}^{e x}=\sum_{i=1}^{N^{e x}} \xi_{i}^{e x} \Phi_{i}^{e x}$ and $\sigma_{h}^{i n}=\sum_{i=1}^{N^{i n}} \xi_{i}^{\text {in }} \Phi_{i}^{i n}$ in (2.1.10) and get one linear system

$$
A \xi:=\left(\begin{array}{ccc}
A_{11} & A_{12} & A_{13} \\
A_{21} & A_{22} & 0 \\
A_{31} & 0 & A_{33}
\end{array}\right)\left(\begin{array}{c}
\xi^{I} \\
\xi^{e x} \\
\xi^{\text {in }}
\end{array}\right)=b
$$

Obviously $A_{11}, A_{22}$ and $A_{33}$ are symmetric and the calculation is the same as $A$ in Section 1.2.5. So we just compare $A_{12}$ with $A_{21}$ and $A_{13}$ with $A_{31}$.

$$
A_{12}[i][j]=\alpha^{e x}\left(\frac{1}{2} \int_{\Gamma^{I}} \Phi_{j}^{e x}(x) \Phi_{i}^{I}(x) \mathrm{d} s_{x}+\int_{\Gamma^{I}} \int_{\Gamma^{e x}} \frac{\partial E_{k^{e x}}(x, y)}{\partial \mathbf{n}_{x}} \Phi_{j}^{e x}(y) \Phi_{i}^{I}(x) \mathrm{d} s_{y} \mathrm{~d} s_{x}\right)
$$




$$
\begin{aligned}
& A_{21}[j][i]=\frac{1}{2} \int_{\Gamma^{I}} \Phi_{i}^{I}(x) \Phi_{j}^{e x}(x) \mathrm{d} s_{x}+\int_{\Gamma^{e x}} \int_{\Gamma^{I}} \frac{\partial E_{k^{e x}}(x, y)}{\partial \mathbf{n}_{y}} \Phi_{i}^{I}(y) \Phi_{j}^{e x}(x) \mathrm{d} s_{y} \mathrm{~d} s_{x} \\
& A_{13}[i][j]=\alpha^{i n}\left(\frac{1}{2} \int_{\Gamma^{I}} \Phi_{j}^{i n}(x) \Phi_{i}^{I}(x) \mathrm{d} s_{x}-\int_{\Gamma^{I}} \int_{\Gamma^{i n}} \frac{\partial E_{k^{i n}}(x, y)}{\partial \mathbf{n}_{x}^{e x}} \Phi_{j}^{i n}(y) \Phi_{i}^{I}(x) \mathrm{d} s_{y} \mathrm{~d} s_{x}\right) \\
& A_{31}[j][i]=\frac{1}{2} \int_{\Gamma^{i n}} \Phi_{i}^{I}(x) \Phi_{j}^{i n}(x) \mathrm{d} s_{x}+\int_{\Gamma^{i n}} \int_{\Gamma^{i n}} \frac{\partial E_{k^{i n}}(x, y)}{\partial \mathbf{n}_{y}^{i n}} \Phi_{i}^{I}(y) \Phi_{j}^{i n}(x) \mathrm{d} s_{y} \mathrm{~d} s_{x}
\end{aligned}
$$

This shows that $A_{12}[i][j]=\alpha^{e x} A_{21}[j][i]$ and $A_{13}[i][j]=\alpha^{i n} A_{31}[j][i]$ results from $\mathbf{n}^{e x}(x)=-\mathbf{n}^{i n}(x)$ for $x \in \Gamma^{I}$.

Now we go to the last step to consider the a Priori error estimates for (2.1.10). The Galerkin discretization for the variation formulation of (2.1.9) is the same with (2.1.10). From [53, Lemma 2.3], we have the coercivity of $S_{k^{e x}}^{\Gamma^{e x}}$ and $S_{k^{i n}}^{\Gamma^{i n}}$, so we can repeat all the work in Section 1.2.5 for (2.1.9) and get the best approximation for $\varphi_{h}^{I}$

$$
\left\|\varphi_{h}^{I}-\varphi^{I}\right\|_{H^{1 / 2}\left(\Gamma^{I}\right)} \leq c \inf _{v_{h}^{I} \in S_{h}^{1}\left(\Gamma^{I}\right)}\left\|v_{h}^{I}-\varphi^{I}\right\|_{H^{1 / 2}\left(\Gamma^{I}\right)} .
$$

If we assume that the solution is regular enough, we can use (1.2.22b) in (2.1.11) and get the quasi-optimal error estimate

$$
\left\|\varphi_{h}^{I}-\varphi^{I}\right\|_{H^{1 / 2}\left(\Gamma^{I}\right)} \leq c h^{3 / 2}\left|\varphi^{I}\right|_{H_{p w}^{2}\left(\Gamma^{I}\right)} .
$$

By using the Aubin-Nitsche duality, we get the super convergence in $L^{2}$-norm

$$
\left\|\varphi_{h}^{I}-\varphi^{I}\right\|_{L^{2}\left(\Gamma^{I}\right)} \leq c h^{2}\left|\varphi^{I}\right|_{H_{p w}^{2}\left(\Gamma^{I}\right)} .
$$

We can use $\varphi_{h}^{I}$ as the given data in (2.1.10b) and (2.1.10c) to calculate $\sigma_{h}^{e x}$ and $\sigma_{h}^{i n}$. The error estimates for $\sigma_{h}^{e x}$ and $\sigma_{h}^{i n}$ are definitely the same as for the Dirichlet boundary value problem in Section 1.2.5. Then we have

$$
\begin{aligned}
& \left\|\sigma_{h}^{e x}-\sigma^{e x}\right\|_{L^{2}\left(\Gamma^{e x}\right)} \leq c h\left|\sigma^{e x}\right|_{H_{p w}^{1}\left(\Gamma^{e x}\right)}, \\
& \left\|\sigma_{h}^{i n}-\sigma^{i n}\right\|_{L^{2}\left(\Gamma^{i n}\right)} \leq c h\left|\sigma^{i n}\right|_{H_{p w}^{1}\left(\Gamma^{i n}\right)} .
\end{aligned}
$$

\subsubsection{Numerical tests}

We consider one example on the boundary of $\Omega^{i n}=(1 / 3,2 / 3)^{3}$ and the boundary of $\Omega^{e x}=(0,1)^{3} \backslash \overline{\Omega^{i n}}$. The analytical solution used for the test is (1.2.26). In Table 2.1 the first column is the level of meshes, the second column is the number of degrees of freedom, the third, fifth and seventh columns are $L^{2}$-error for $\sigma_{h}^{e x}, \varphi_{h}^{I}$ and $\sigma_{h}^{i n}$ respectively. The fourth, sixth and eighth columns are the rate of convergence $(s)$ in $O\left(h^{s}\right)$. Fig. 2.1a, $2.1 \mathrm{~b}$ and 2.1c are the results of $\sigma_{h}^{e x}, \varphi_{h}^{I}$ and $\sigma_{h}^{i n}$ respectively. The calculation of $L^{2}$-error and the rate of convergence is the same as in Section 1.2.6.

In the fourth and eighth columns we observe a linear convergence for piecewise constant function spaces and in the sixth column we observe a quadratic convergence for piecewise linear function spaces approximately. The perturbation in the rate of convergence is the same reasons as in Chapter 1. 


\begin{tabular}{|c|c|c|c|c|c|c|c|}
\hline level & dof & $\left\|\sigma_{h}^{e x}-\sigma^{e x}\right\|_{L^{2}}$ & $\mathrm{CR}$ & $\left\|\varphi_{h}^{I}-\varphi^{I}\right\|_{L^{2}}$ & $\mathrm{CR}$ & $\left\|\sigma_{h}^{i n}-\sigma^{i n}\right\|_{L^{2}}$ & $\mathrm{CR}$ \\
\hline 0 & 278 & 1.36232 & - & 0.03133 & - & 0.27695 & - \\
\hline 1 & 1106 & 0.52224 & 1.3833 & 0.00690 & 2.1827 & 0.13659 & 1.0197 \\
\hline 2 & 4418 & 0.21007 & 1.3138 & 0.00163 & 2.0813 & 0.05520 & 1.3071 \\
\hline
\end{tabular}

Table 2.1 Accuracy of Galerkin-BEMs for interface problem with Dirichlet boundary condition for the Helmholtz equation

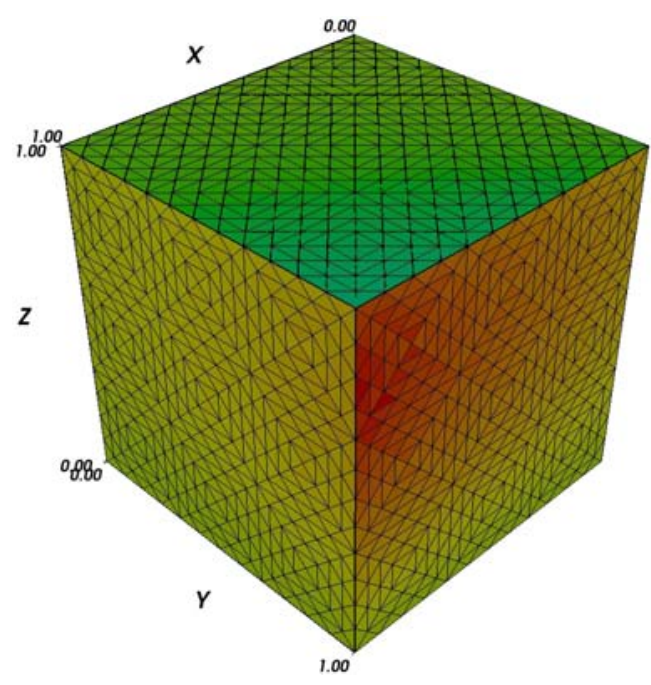

(a) $\sigma^{e x}$, Neumann trace from exterior domain

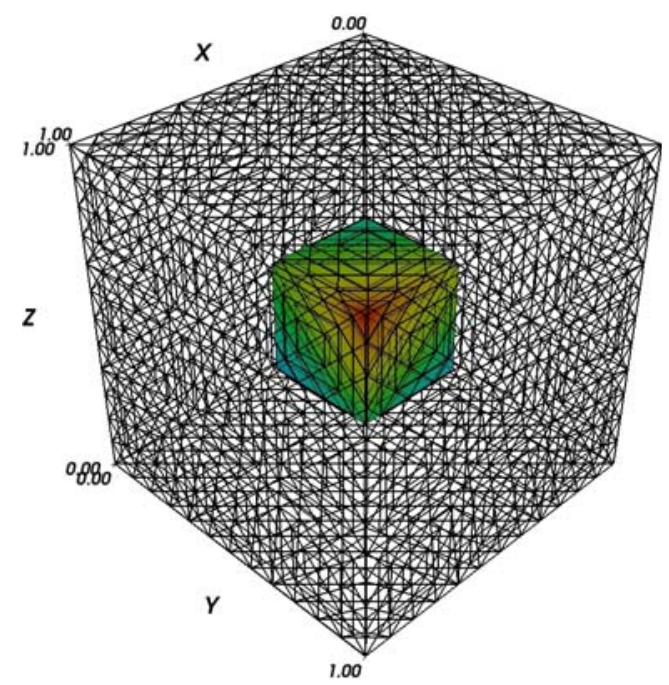

(b) $\varphi^{I}$, Dirichlet trace on interface

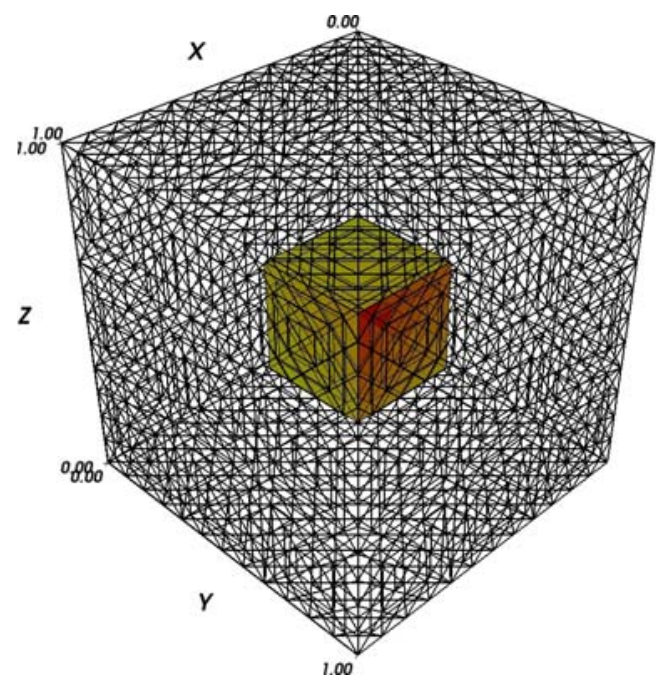

(c) $\sigma^{i n}$, Neumann trace from interior domain

Figure 2.1 Interface problem with Dirichlet boundary condition for the Helmholtz equation 


\subsection{The Maxwell case}

\subsubsection{Interface problem}

In this section we consider electromagnetic waves in an inhomogeneous dielectric medium in $\Omega:=\Omega^{e x} \cup \overline{\Omega^{i n}}$. The medium has different permeability and permittivity in $\Omega^{e x}$ and $\Omega^{i n}$ respectively. The time-harmonic Maxwell's equations for electric or magnetic fields with a Dirichlet boundary condition are

$$
\begin{array}{lc}
\nabla \times\left(\frac{1}{\alpha(x)} \nabla \times \mathbf{u}(x)\right)-k^{2} \mathbf{u}(x)=0 & \forall x \in \Omega^{e x} \bigcup \Omega^{i n}, \\
\nabla \cdot \mathbf{u}(x)=0 & \forall x \in \Omega^{e x} \bigcup \Omega^{i n}, \\
\gamma_{\mathbf{t}}(\mathbf{u})(x)=\mathbf{f}(x) & \forall x \in \Gamma:=\partial \Omega
\end{array}
$$

where $k$ is a positive real number, $\alpha=\alpha^{e x}=\varepsilon^{e x} \mu^{e x}$ in $\Omega^{e x}, \alpha=\alpha^{i n}=\varepsilon^{i n} \mu^{i n}$ in $\Omega^{i n}$, and $\mathbf{f}$ is the given data. $\varepsilon^{e x}, \mu^{e x}, \varepsilon^{i n}, \mu^{i n}$ are positive constants.

We have two interface conditions for this problem from (1.1.2b) and (1.1.2d),

$$
\begin{array}{ll}
\gamma_{\mathbf{t}}^{e x}\left(\mathbf{u}^{e x}\right)(x)+\gamma_{\mathbf{t}}^{i n}\left(\mathbf{u}^{i n}\right)(x)=0 & \forall x \in \Gamma^{I}, \\
\beta^{e x} \gamma_{\mathbf{N}}^{e x}\left(\mathbf{u}^{e x}\right)(x)+\beta^{i n} \gamma_{\mathbf{N}}^{i n}\left(\mathbf{u}^{i n}\right)(x)=0 & \forall x \in \Gamma^{I},
\end{array}
$$

where $\mathbf{u}^{e x}:=\left.\mathbf{u}\right|_{\overline{\Omega^{e x}}}, \mathbf{u}^{i n}:=\left.\mathbf{u}\right|_{\overline{\Omega^{i n}}}, \gamma_{\mathbf{t}}^{e x}, \gamma_{\mathbf{t}}^{i n}, \gamma_{\mathbf{N}}^{e x}, \gamma_{\mathbf{N}}^{i n}$ are Dirichlet and Neumann trace operators from $\Omega^{e x}$ and $\Omega^{i n}$, respectively, $\beta^{e x}=\sqrt{\frac{\varepsilon^{e x}}{\mu^{e x}}}, \beta^{i n}=\sqrt{\frac{\varepsilon^{i n}}{\mu^{i n}}}$ for electric fields, and $\Gamma^{I}:=\Gamma^{e x} \bigcap \Gamma^{i n}$ with $\Gamma^{e x}:=\partial \Omega^{e x}$ and $\Gamma^{i n}:=\partial \Omega^{i n}$. The definitions of the Dirichlet and Neumann trace operators could be found in Section 1.3.3.

\subsubsection{Domain decomposition method}

We define $\boldsymbol{\sigma}^{e x}:=\gamma_{\mathbf{N}}^{e x}\left(\mathbf{u}^{e x}\right), \boldsymbol{\sigma}^{i n}:=\gamma_{\mathbf{N}}^{i n}\left(\mathbf{u}^{i n}\right), \varphi^{e x}:=\gamma_{\mathbf{t}}^{e x}\left(\mathbf{u}^{e x}\right)$ and $\varphi^{i n}:=\gamma_{\mathbf{t}}^{i n}\left(\mathbf{u}^{i n}\right)$. From (1.3.14) we could define two Calderon projections for $\left(\boldsymbol{\sigma}^{e x}, \varphi^{e x}\right)$ and $\left(\boldsymbol{\sigma}^{i n}, \boldsymbol{\varphi}^{i n}\right)$, respectively, as

$$
\begin{aligned}
\left(\begin{array}{l}
\boldsymbol{\varphi}^{e x} \\
\boldsymbol{\sigma}^{e x}
\end{array}\right) & =\left(\begin{array}{cc}
\frac{1}{2} I+\mathbf{C}_{k^{e x}}^{\Gamma^{e x}} & \mathbf{S}_{k^{e x}}^{\Gamma^{e x}} \\
\mathbf{S}_{k^{e x}}^{\Gamma^{e x}} & \frac{1}{2} I+\mathbf{C}_{k^{e x}}^{\Gamma^{e x}}
\end{array}\right)\left(\begin{array}{l}
\boldsymbol{\varphi}^{e x} \\
\boldsymbol{\sigma}^{e x}
\end{array}\right), \\
\left(\begin{array}{c}
\boldsymbol{\varphi}^{i n} \\
\boldsymbol{\sigma}^{i n}
\end{array}\right) & =\left(\begin{array}{cc}
\frac{1}{2} I+\mathbf{C}_{k^{i n}}^{\Gamma^{i n}} & \mathbf{S}_{k^{i n}}^{\Gamma^{i n}} \\
\mathbf{S}_{k^{i n}}^{\Gamma^{i n}} & \frac{1}{2} I+\mathbf{C}_{k^{i n}}^{\Gamma^{i n}}
\end{array}\right)\left(\begin{array}{l}
\boldsymbol{\varphi}^{i n} \\
\boldsymbol{\sigma}^{i n}
\end{array}\right),
\end{aligned}
$$

where $k^{e x}=\sqrt{\alpha^{e x}} k, k^{i n}=\sqrt{\alpha^{i n}} k$, and $\mathbf{S}_{k^{e x}}^{\Gamma^{e x}}, \mathbf{C}_{k^{e x}}^{\Gamma^{e x}}, \mathbf{S}_{k^{i n}}^{\Gamma^{i n}}, \mathbf{C}_{k^{i n}}^{\Gamma^{i n}}$ are boundary integral operators on $\Gamma^{e x}$ and $\Gamma^{i n}$, respectively, with the definitions in Section 1.3.3.

We use the second equations of (2.2.3a) and (2.2.3b) in (2.2.2b) and get

$$
\begin{aligned}
\beta^{e x}\left(\mathbf{S}_{k^{e x}}^{\Gamma^{e x}}\left(\boldsymbol{\varphi}^{e x}\right)+\right. & \left.\left(\frac{1}{2} I+\mathbf{C}_{k^{e x}}^{\Gamma^{e x}}\right)\left(\boldsymbol{\sigma}^{e x}\right)\right) \\
& +\beta^{i n}\left(\mathbf{S}_{k^{i n}}^{\Gamma^{i n}}\left(\boldsymbol{\varphi}^{i n}\right)+\left(\frac{1}{2} I+\mathbf{C}_{k^{i n}}^{\Gamma^{i n}}\right)\left(\boldsymbol{\sigma}^{i n}\right)\right)=0 .
\end{aligned}
$$


From (2.2.2a) and (2.2.1c) we have $\varphi^{e x}=-\varphi^{i n}$ on $\Gamma^{I}$ and $\varphi^{e x}=\mathbf{f}$ on $\Gamma$. We define $\varphi^{I}$ as $\varphi^{I}=\varphi^{e x}=-\varphi^{i n}$ on $\Gamma^{I}$ and $\varphi^{I}=0$ on $\Gamma$. We also assume that the extension of $\mathrm{f}$ is zero on $\Gamma^{I}$. We assume that the exterior unit normal on $\Gamma^{I}$ and $\Gamma$ is the same as on $\Gamma^{e x}$. We use $\varphi^{I}$ and $\mathbf{f}$ in (2.2.4) and get

$$
\begin{aligned}
\left(\beta^{e x} \mathbf{S}_{k^{e x}}^{\Gamma^{I}}+\right. & \left.\beta^{i n} \mathbf{S}_{k^{i n}}^{\Gamma^{I}}\right)\left(\boldsymbol{\varphi}^{I}\right)(x)+\beta^{e x}\left(\frac{1}{2} I+\mathbf{C}_{k^{e x}}^{\Gamma^{e x}}\right)\left(\boldsymbol{\sigma}^{e x}\right)(x) \\
& +\beta^{i n}\left(\frac{1}{2} I+\mathbf{C}_{k^{i n}}^{\Gamma^{i n}}\right)\left(\boldsymbol{\sigma}^{i n}\right)(x)=-\beta^{e x} \mathbf{S}_{k^{e x}}^{\Gamma}(\mathbf{f})(x)
\end{aligned} \quad \forall x \in \Gamma^{I}
$$

Notice that we change the operator $\mathbf{S}_{k^{i n}}^{\Gamma^{i n}}$ to $-\mathbf{S}_{k^{i n}}^{\Gamma^{I}}$ in (2.2.5). From the first equations in (2.2.3a) and (2.2.3b) we get

$$
\begin{aligned}
& \left(-\frac{1}{2} I+\mathbf{C}_{k^{e x}}^{\Gamma^{I}}\right)\left(\boldsymbol{\varphi}^{I}\right)(x)+\mathbf{S}_{k^{e x}}^{\Gamma^{e x}}\left(\boldsymbol{\sigma}^{e x}\right)(x) \\
& =-\left(-\frac{1}{2} I+\mathbf{C}_{k^{e x}}^{\Gamma}\right)(\mathbf{f})(x) \quad \forall x \in \Gamma^{e x}, \\
& \left(\frac{1}{2} I-\mathbf{C}_{k^{i n}}^{\Gamma^{i n}}\right)\left(\boldsymbol{\varphi}^{I}\right)(x)+\mathbf{S}_{k^{i n}}^{\Gamma^{i n}}\left(\boldsymbol{\sigma}^{i n}\right)(x)=0 \quad \forall x \in \Gamma^{i n} .
\end{aligned}
$$

The combination of (2.2.5), (2.2.6) and (2.2.7) is the system of equations for the unknown functions $\left(\varphi^{I}, \sigma^{e x}, \sigma^{i n}\right)$. The solution on the boundary of the whole domain has been changed to the solution on the boundaries of the subdomains.

\subsubsection{Variational formulation}

By using the left hand side of (2.2.5), (2.2.6) and (2.2.7) we define three operators

$$
\begin{aligned}
& \mathbf{F}_{1}: \mathbf{W}^{-1 / 2}\left(\Gamma^{I}\right) \times \mathbf{W}^{-1 / 2}\left(\Gamma^{e x}\right) \times \mathbf{W}^{-1 / 2}\left(\Gamma^{i n}\right) \rightarrow \mathbf{W}^{-1 / 2}\left(\Gamma^{I}\right), \\
& \mathbf{F}_{2}: \mathbf{W}^{-1 / 2}\left(\Gamma^{I}\right) \times \mathbf{W}^{-1 / 2}\left(\Gamma^{e x}\right) \rightarrow \mathbf{W}^{-1 / 2}\left(\Gamma^{e x}\right), \\
& \mathbf{F}_{3}: \mathbf{W}^{-1 / 2}\left(\Gamma^{I}\right) \times \mathbf{W}^{-1 / 2}\left(\Gamma^{i n}\right) \rightarrow \mathbf{W}^{-1 / 2}\left(\Gamma^{i n}\right),
\end{aligned}
$$

which results into the following variational formulation.

Find $\left(\boldsymbol{\varphi}^{I}, \boldsymbol{\sigma}^{e x}, \boldsymbol{\sigma}^{i n}\right) \in \mathbf{W}^{-1 / 2}\left(\Gamma^{I}\right) \times \mathbf{W}^{-1 / 2}\left(\Gamma^{e x}\right) \times \mathbf{W}^{-1 / 2}\left(\Gamma^{i n}\right)$ such that

$$
\begin{aligned}
& \left\langle\mathbf{F}_{1}\left(\boldsymbol{\varphi}^{I}, \boldsymbol{\sigma}^{e x}, \boldsymbol{\sigma}^{i n}\right), \boldsymbol{v}^{1}\right\rangle_{\tau, \Gamma^{I}}=\left\langle-\beta^{e x} \mathbf{S}_{k^{e x}}^{\Gamma}(\mathbf{f}), \boldsymbol{v}^{1}\right\rangle_{\tau, \Gamma^{I}}, \\
& \left\langle\mathbf{F}_{2}\left(\boldsymbol{\varphi}^{I}, \boldsymbol{\sigma}^{e x}\right), \boldsymbol{v}^{2}\right\rangle_{\tau, \Gamma^{e x}}=\left\langle\left(\frac{1}{2} I-\mathbf{C}_{k^{e x}}^{\Gamma}\right)(\mathbf{f}), \boldsymbol{v}^{2}\right\rangle_{\tau, \Gamma^{e x}}, \\
& \left\langle\mathbf{F}_{3}\left(\boldsymbol{\varphi}^{I}, \boldsymbol{\sigma}^{i n}\right), \boldsymbol{v}^{3}\right\rangle_{\tau, \Gamma^{i n}}=0,
\end{aligned}
$$

for all $\left(\boldsymbol{v}^{1}, \boldsymbol{v}^{2}, \boldsymbol{v}^{3}\right) \in \mathbf{W}^{-1 / 2}\left(\Gamma^{I}\right) \times \mathbf{W}^{-1 / 2}\left(\Gamma^{e x}\right) \times \mathbf{W}^{-1 / 2}\left(\Gamma^{i n}\right)$.

For the existence and uniqueness of the solution of (2.2.8), we need a generalized Gårding inequality as in Section 1.3.4. The basic ideas of the proof have been given in [13, Theorem 9] for the transmission problem. We follow their ideas and give a proof for 
our problem (2.2.8). We define an operator $\mathbf{F}$ as

$$
\mathbf{F}\left(\begin{array}{c}
\mathbf{v}_{1} \\
\mathbf{v}_{2} \\
\mathbf{v}_{3}
\end{array}\right)=\left(\begin{array}{c}
\mathbf{F}_{1}\left(\mathbf{v}_{1}, \mathbf{v}_{2}, \mathbf{v}_{3}\right) \\
\beta^{e x} \mathbf{F}_{2}\left(\mathbf{v}_{1}, \mathbf{v}_{2}\right) \\
\beta^{\text {in }} \mathbf{F}_{3}\left(\mathbf{v}_{1}, \mathbf{v}_{3}\right)
\end{array}\right)
$$

for $\left(\mathbf{v}_{1}, \mathbf{v}_{2}, \mathbf{v}_{3}\right) \in \mathbf{W}^{-1 / 2}\left(\Gamma^{I}\right) \times \mathbf{W}^{-1 / 2}\left(\Gamma^{e x}\right) \times \mathbf{W}^{-1 / 2}\left(\Gamma^{i n}\right)$. The reason to multiply by $\beta^{e x}$ and $\beta^{i n}$ is to have a symmetry of $\mathbf{F}$. We also define a sesquilinear pairing for $\mathbf{F}$ as

$$
\left\langle\mathbf{F}\left(\begin{array}{c}
\mathbf{v}_{1} \\
\mathbf{v}_{2} \\
\mathbf{v}_{3}
\end{array}\right),\left(\begin{array}{c}
\mathbf{w}_{1} \\
\mathbf{w}_{2} \\
\mathbf{w}_{3}
\end{array}\right)\right\rangle_{\tau} \begin{aligned}
& \left\langle\mathbf{F}_{1}\left(\mathbf{v}_{1}, \mathbf{v}_{2}, \mathbf{v}_{3}\right), \mathbf{w}_{1}\right\rangle_{\tau, \Gamma^{I}} \\
& +\left\langle\mathbf{F}_{2}\left(\mathbf{v}_{1}, \mathbf{v}_{2}\right), \mathbf{w}_{2}\right\rangle_{\tau, \Gamma^{e x}} \\
& +\left\langle\mathbf{F}_{3}\left(\mathbf{v}_{1}, \mathbf{v}_{3}\right), \mathbf{w}_{3}\right\rangle_{\tau, \Gamma^{i n}}
\end{aligned} .
$$

We define an operator $\mathbf{X}$ as

$$
\mathbf{X}\left(\begin{array}{l}
\mathbf{v}_{1} \\
\mathbf{v}_{2} \\
\mathbf{v}_{3}
\end{array}\right)=\left(\begin{array}{c}
X^{\Gamma^{I}}\left(\mathbf{v}_{1}\right) \\
X^{\Gamma^{e x}}\left(\mathbf{v}_{2}\right) \\
X^{\Gamma^{i n}}\left(\mathbf{v}_{3}\right)
\end{array}\right)
$$

where the definitions of $X^{\Gamma^{I}}, X^{\Gamma^{e x}}$ and $X^{\Gamma^{i n}}$ can be found in Lemma 9 in Section 1.3.4. Then we have a lemma for the generalized Gårding inequality for $\mathbf{F}$.

Lemma 12. There exists a compact bilinear form $\mathbf{c}(\cdot, \cdot)$ such that $\mathbf{F}$ satisfies the generalized Gårding inequality

$$
\begin{aligned}
& \left\langle\mathbf{F}\left(\begin{array}{l}
\mathbf{v}_{1} \\
\mathbf{v}_{2} \\
\mathbf{v}_{3}
\end{array}\right), \mathbf{X}\left(\begin{array}{c}
\mathbf{v}_{1} \\
\mathbf{v}_{2} \\
\mathbf{v}_{3}
\end{array}\right)\right\rangle_{\tau}+\mathbf{c}\left(\left(\begin{array}{c}
\mathbf{v}_{1} \\
\mathbf{v}_{2} \\
\mathbf{v}_{3}
\end{array}\right),\left(\begin{array}{l}
\mathbf{v}_{1} \\
\mathbf{v}_{2} \\
\mathbf{v}_{3}
\end{array}\right)\right) \\
& \geq c\left(\left\|\mathbf{v}_{1}\right\|_{\mathbf{W}^{-1 / 2}\left(\Gamma^{I}\right)}^{2}+\left\|\mathbf{v}_{2}\right\|_{\mathbf{W}^{-1 / 2}\left(\Gamma^{e x}\right)}^{2}+\left\|\mathbf{v}_{3}\right\|_{\mathbf{W}^{-1 / 2}\left(\Gamma^{i n}\right)}^{2}\right) .
\end{aligned}
$$

Proof. First we consider the left hand side of the inequality and get

$$
\begin{aligned}
\left\langle\mathbf{F}\left(\begin{array}{c}
\mathbf{v}_{1} \\
\mathbf{v}_{2} \\
\mathbf{v}_{3}
\end{array}\right), \mathbf{X}\left(\begin{array}{c}
\mathbf{v}_{1} \\
\mathbf{v}_{2} \\
\mathbf{v}_{3}
\end{array}\right)\right\rangle_{\tau} & \left\langle\mathbf{F}_{1}\left(\mathbf{v}_{1}, \mathbf{v}_{2}, \mathbf{v}_{3}\right), X^{\Gamma^{I}}\left(\mathbf{v}_{1}\right)\right\rangle_{\tau, \Gamma^{I}} \\
& +\left\langle\mathbf{F}_{3}\left(\mathbf{v}_{1}, \mathbf{v}_{3}\right), X^{\Gamma^{i n}}\left(\mathbf{v}_{3}\right)\right\rangle_{\tau, \Gamma^{i n}} \\
= & \left\langle\left(\beta^{e x} \mathbf{S}_{k^{e x}}^{\Gamma^{I}}+\beta^{i n} \mathbf{S}_{k^{i n}}^{\Gamma^{I}}\right)\left(\mathbf{v}_{1}\right)+\beta^{e x}\left(\frac{1}{2} I+\mathbf{C}_{k^{e x}}^{\Gamma^{e x}}\right)\left(\mathbf{v}_{2}\right)\right. \\
& \left.+\beta^{i n}\left(\frac{1}{2} I+\mathbf{C}_{k^{i n}}^{\Gamma^{i n}}\right)\left(\mathbf{v}_{3}\right), X^{\Gamma^{I}}\left(\mathbf{v}_{1}\right)\right\rangle_{\tau, \Gamma^{I}} \\
& +\beta^{e x}\left\langle\left(-\frac{1}{2} I+\mathbf{C}_{k^{e x}}^{\Gamma^{I}}\right)\left(\mathbf{v}_{1}\right)+\mathbf{S}_{k^{e x}}^{\Gamma^{e x}}\left(\mathbf{v}_{2}\right), X^{\Gamma^{e x}}\left(\mathbf{v}_{2}\right)\right\rangle_{\tau, \Gamma^{e x}} \\
& +\beta^{i n}\left\langle\left(\frac{1}{2} I-\mathbf{C}_{k^{i n}}^{\Gamma^{i n}}\right)\left(\mathbf{v}_{1}\right)+\mathbf{S}_{k^{i n}}^{\Gamma^{i n}}\left(\mathbf{v}_{3}\right), X^{\Gamma^{i n}}\left(\mathbf{v}_{3}\right)\right\rangle_{\tau, \Gamma^{i n}} .
\end{aligned}
$$

From Lemma 9 we have the coercivity of $\mathbf{S}_{k^{e x}}^{\Gamma^{I}}, \mathbf{S}_{k^{i n}}^{\Gamma^{I}}, \mathbf{S}_{k^{e x}}^{\Gamma^{e x}}$ and $\mathbf{S}_{k^{i n}}^{\Gamma^{i n}}$, so we just need to 
consider the double-layer boundary integral operators. We consider the second and fourth terms on the right hand side of the above formulation as the following

$$
\left\langle\beta^{e x}\left(\frac{1}{2} I+\mathbf{C}_{k^{e x}}^{\Gamma^{e x}}\right)\left(\mathbf{v}_{2}\right), X^{\Gamma^{I}}\left(\mathbf{v}_{1}\right)\right\rangle_{\tau, \Gamma^{I}}+\beta^{e x}\left\langle\left(-\frac{1}{2} I+\mathbf{C}_{k^{e x}}^{\Gamma^{I}}\right)\left(\mathbf{v}_{1}\right), X^{\Gamma^{e x}}\left(\mathbf{v}_{2}\right)\right\rangle_{\tau, \Gamma^{e x}},
$$

and the argument for the third and sixth terms is the same.

By using Lemma 6 in [13] for the double-layer operators in (2.2.9), we have

$$
\begin{aligned}
& \left\langle\mathbf{C}_{k^{e x}}^{\Gamma^{e x}}\left(\mathbf{v}_{2}\right), X^{\Gamma^{I}}\left(\mathbf{v}_{1}\right)\right\rangle_{\tau, \Gamma^{I}}+\left\langle\mathbf{C}_{k^{e x}}^{\Gamma^{I}}\left(\mathbf{v}_{1}\right), X^{\Gamma^{e x}}\left(\mathbf{v}_{2}\right)\right\rangle_{\tau, \Gamma^{e x}} \\
\underbrace{=}_{\text {Lemma } 6} & \left\langle\mathbf{C}_{k^{e x}}^{\Gamma^{I}}\left(X^{\Gamma^{I}}\left(\mathbf{v}_{1}\right)\right), \mathbf{v}_{2}\right\rangle_{\tau, \Gamma^{e x}}+\left\langle\mathbf{C}_{k^{e x}}^{\Gamma^{I}}\left(\mathbf{v}_{1}\right), X^{\Gamma^{e x}}\left(\mathbf{v}_{2}\right)\right\rangle_{\tau, \Gamma^{e x}} \\
= & \left\langle\mathbf{C}_{k^{e x}}^{\Gamma^{I}}\left(R^{\Gamma^{I}}\left(\mathbf{v}_{1}\right)-Z^{\Gamma^{I}}\left(\mathbf{v}_{1}\right)\right), R^{\Gamma^{e x}}\left(\mathbf{v}_{2}\right)+Z^{\Gamma^{e x}}\left(\mathbf{v}_{2}\right)\right\rangle_{\tau, \Gamma^{e x}} \\
& +\left\langle\mathbf{C}_{k^{e x}}^{\Gamma^{I}}\left(R^{\Gamma^{I}}\left(\mathbf{v}_{1}\right)+Z^{\Gamma^{I}}\left(\mathbf{v}_{1}\right)\right), R^{\Gamma^{e x}}\left(\mathbf{v}_{2}\right)-Z^{\Gamma^{e x}}\left(\mathbf{v}_{2}\right)\right\rangle_{\tau, \Gamma^{e x}} \\
= & 2\left\langle\mathbf{C}_{k^{e x}}^{\Gamma^{I}}\left(R^{\Gamma^{I}}\left(\mathbf{v}_{1}\right)\right), R^{\Gamma^{e x}}\left(\mathbf{v}_{2}\right)\right\rangle_{\tau, \Gamma^{e x}}-2\left\langle\mathbf{C}_{k^{e x}}^{\Gamma^{I}}\left(Z^{\Gamma^{I}}\left(\mathbf{v}_{1}\right)\right), Z^{\Gamma^{e x}}\left(\mathbf{v}_{2}\right)\right\rangle_{\tau, \Gamma^{e x}}
\end{aligned}
$$

By Lemma 12 in [13], we know that $\left\langle\mathbf{C}_{k^{e x}}^{\Gamma^{I}}, \cdot\right\rangle_{\tau, \Gamma^{e x}}$ on $R^{\Gamma^{e x}}\left(\mathbf{W}^{-1 / 2}\left(\Gamma^{e x}\right)\right)^{2}$ and $\left\langle\mathbf{C}_{k^{e x}}^{\Gamma^{I}} \cdot, \cdot\right\rangle_{\tau, \Gamma^{e x}}$ on $Z^{\Gamma^{e x}}\left(\mathbf{W}^{-1 / 2}\left(\Gamma^{e x}\right)\right)^{2}$ are compact bilinear forms. For the left terms in (2.2.9), we have

$$
\begin{aligned}
& \left\langle\mathbf{v}_{2}, X^{\Gamma^{I}}\left(\mathbf{v}_{1}\right)\right\rangle_{\tau, \Gamma^{I}}-\left\langle\mathbf{v}_{1}, X^{\Gamma^{e x}}\left(\mathbf{v}_{2}\right)\right\rangle_{\tau, \Gamma^{I}} \\
= & \left\langle\mathbf{v}_{2}, X^{\Gamma^{I}}\left(\mathbf{v}_{1}\right)\right\rangle_{\tau, \Gamma^{I}}+\left\langle X^{\Gamma^{e x}}\left(\mathbf{v}_{2}\right), \mathbf{v}_{1}\right\rangle_{\tau, \Gamma^{I}} \\
= & \left\langle R^{\Gamma^{e x}}\left(\mathbf{v}_{2}\right)+Z^{\Gamma^{e x}}\left(\mathbf{v}_{2}\right), R^{\Gamma^{I}}\left(\mathbf{v}_{1}\right)-Z^{\Gamma^{I}}\left(\mathbf{v}_{1}\right)\right\rangle_{\tau, \Gamma^{I}} \\
& +\left\langle R^{\Gamma^{e x}}\left(\mathbf{v}_{2}\right)-Z^{\Gamma^{e x}}\left(\mathbf{v}_{2}\right), R^{\Gamma^{I}}\left(\mathbf{v}_{1}\right)+Z^{\Gamma^{I}}\left(\mathbf{v}_{1}\right)\right\rangle_{\tau, \Gamma^{I}} \\
= & 2\left\langle R^{\Gamma^{e x}}\left(\mathbf{v}_{2}\right), R^{\Gamma^{I}}\left(\mathbf{v}_{1}\right)\right\rangle_{\tau, \Gamma^{I}}-2\left\langle Z^{\Gamma^{e x}}\left(\mathbf{v}_{2}\right), Z^{\Gamma^{I}}\left(\mathbf{v}_{1}\right)\right\rangle_{\tau, \Gamma^{I}} .
\end{aligned}
$$

By Corollary 1 in [13], we know that $\left\langle R^{\Gamma^{e x}} \cdot, R^{\Gamma^{I}} \cdot\right\rangle_{\tau, \Gamma^{I}}$ and $\left\langle Z^{\Gamma^{e x}} \cdot, Z^{\Gamma^{I}} \cdot\right\rangle_{\tau, \Gamma^{I}}$ are compact bilinear forms. So with the coercivity of the single-layer operators we can define a compact bilinear form. The proof is done.

If we assume that $k$ is not an eigenvalue, we have the existence and uniqueness of the solution of (2.2.8) by Theorem 4 in [13] and the Fredholm alternative.

\subsubsection{Galerkin-BEM}

The discretization of (2.2.8) is to find $\left(\boldsymbol{\varphi}_{h}^{I}, \boldsymbol{\sigma}_{h}^{e x}, \boldsymbol{\sigma}_{h}^{i n}\right) \in \mathbf{W}_{h}^{-1 / 2}\left(\Gamma_{h}^{I}\right) \times \mathbf{W}_{h}^{-1 / 2}\left(\Gamma_{h}^{e x}\right) \times$ $\mathbf{W}_{h}^{-1 / 2}\left(\Gamma_{h}^{i n}\right)$ such that

$$
\begin{aligned}
& \left\langle\mathbf{F}_{1}\left(\boldsymbol{\varphi}_{h}^{I}, \boldsymbol{\sigma}_{h}^{e x}, \boldsymbol{\sigma}_{h}^{i n}\right), \boldsymbol{v}_{h}^{1}\right\rangle_{\tau, \Gamma^{I}}=\left\langle-\beta^{e x} \mathbf{S}_{k^{e x}}^{\Gamma}(\mathbf{f}), \boldsymbol{v}_{h}^{1}\right\rangle_{\tau, \Gamma^{I}}, \\
& \left\langle\mathbf{F}_{2}\left(\boldsymbol{\varphi}_{h}^{I}, \boldsymbol{\sigma}_{h}^{e x}\right), \boldsymbol{v}_{h}^{2}\right\rangle_{\tau, \Gamma^{e x}}=\left\langle\left(\frac{1}{2} I-\mathbf{C}_{k^{e x}}^{\Gamma}\right)(\mathbf{f}), \boldsymbol{v}_{h}^{2}\right\rangle_{\tau, \Gamma^{e x}}, \\
& \left\langle\mathbf{F}_{3}\left(\boldsymbol{\varphi}_{h}^{I}, \boldsymbol{\sigma}_{h}^{i n}\right), \boldsymbol{v}_{h}^{3}\right\rangle_{\tau, \Gamma^{i n}}=0
\end{aligned}
$$


for all $\left(\boldsymbol{v}_{h}^{1}, \boldsymbol{v}_{h}^{2}, \boldsymbol{v}_{h}^{3}\right) \in \mathbf{W}_{h}^{-1 / 2}\left(\Gamma_{h}^{I}\right) \times \mathbf{W}_{h}^{-1 / 2}\left(\Gamma_{h}^{e x}\right) \times \mathbf{W}_{h}^{-1 / 2}\left(\Gamma_{h}^{i n}\right)$. The definitions of $\mathbf{W}_{h}^{-1 / 2}\left(\Gamma_{h}^{I}\right), \mathbf{W}_{h}^{-1 / 2}\left(\Gamma_{h}^{e x}\right)$ and $\mathbf{W}_{h}^{-1 / 2}\left(\Gamma_{h}^{i n}\right)$ could be found in Section 1.3.5.

Let $\left\{\boldsymbol{\Phi}_{i}^{I}\right\}_{i=1}^{N^{I}},\left\{\boldsymbol{\Phi}_{i}^{e x}\right\}_{i=1}^{N^{e x}}$ and $\left\{\boldsymbol{\Phi}_{i}^{i n}\right\}_{i=1}^{N^{i n}}$ be the basis of $\mathbf{W}_{h}^{-1 / 2}\left(\Gamma_{h}^{I}\right), \mathbf{W}_{h}^{-1 / 2}\left(\Gamma_{h}^{e x}\right)$ and $\mathbf{W}_{h}^{-1 / 2}\left(\Gamma_{h}^{i n}\right)$ respectively. We use $\boldsymbol{\varphi}_{h}^{I}=\sum_{j=1}^{N^{I}} \xi_{j}^{I} \boldsymbol{\Phi}_{j}^{I}, \boldsymbol{\varphi}_{h}^{e x}=\sum_{j=1}^{N^{e x}} \xi_{j}^{e x} \boldsymbol{\Phi}_{j}^{e x}$ and $\boldsymbol{\varphi}_{h}^{i n}=$ $\sum_{j=1}^{N^{i n}} \xi_{j}^{i n} \boldsymbol{\Phi}_{j}^{i n}$ in (2.2.10) and get one linear system

$$
A \xi:=\left(\begin{array}{ccc}
A_{11} & A_{12} & A_{13} \\
A_{21} & A_{22} & 0 \\
A_{31} & 0 & A_{33}
\end{array}\right)\left(\begin{array}{c}
\xi^{I} \\
\xi^{e x} \\
\xi^{\text {in }}
\end{array}\right)=b .
$$

Obviously $A_{11}, A_{22}$ and $A_{33}$ are symmetric and the calculation is the same as $A$ in Section 1.3.5. So we just compare $A_{12}$ and $A_{13}$ with $A_{21}$ and $A_{31}$.

$$
\begin{aligned}
\frac{A_{12}[i, j]}{\beta^{e x}}= & \frac{1}{2} \int_{\Gamma^{I}} \boldsymbol{\Phi}_{j}^{e x}(x) \times \mathbf{n}(x) \cdot \boldsymbol{\Phi}_{i}^{I}(x) \mathrm{d} s_{x} \\
& +\int_{\Gamma^{I}} \int_{\Gamma^{e x} \backslash\{x\}} \nabla_{x} E_{k}(x, y) \cdot\left(\boldsymbol{\Phi}_{j}^{e x}(y) \times \boldsymbol{\Phi}_{i}^{I}(x)\right) \mathrm{d} s_{y} \mathrm{~d} s_{x} \\
A_{21}[j, i]= & -\frac{1}{2} \int_{\Gamma^{I}} \boldsymbol{\Phi}_{i}^{I}(x) \times \mathbf{n}(x) \cdot \boldsymbol{\Phi}_{j}^{e x}(x) \mathrm{d} s_{x} \\
& +\int_{\Gamma^{e x}} \int_{\Gamma^{I} \backslash\{x\}} \nabla_{x} E_{k}(x, y) \cdot\left(\boldsymbol{\Phi}_{i}^{I}(y) \times \boldsymbol{\Phi}_{j}^{e x}(x)\right) \mathrm{d} s_{y} \mathrm{~d} s_{x} \\
\frac{A_{13}[i, j]}{\beta^{i n}=} & \frac{1}{2} \int_{\Gamma^{I}} \boldsymbol{\Phi}_{j}^{I}(x) \times \mathbf{n}(x) \cdot \boldsymbol{\Phi}_{i}^{I}(x) \mathrm{d} s_{x} \\
& -\int_{\Gamma^{I}} \int_{\Gamma^{I} \backslash\{x\}} \nabla_{x} E_{k}(x, y) \cdot\left(\boldsymbol{\Phi}_{j}^{I}(y) \times \boldsymbol{\Phi}_{i}^{I}(x)\right) \mathrm{d} s_{y} \mathrm{~d} s_{x} \\
A_{31}[j, i]= & \frac{1}{2} \int_{\Gamma^{i n}} \boldsymbol{\Phi}_{i}^{I}(x) \times \mathbf{n}(x) \cdot \boldsymbol{\Phi}_{j}^{I}(x) \mathrm{d} s_{x} \\
& -\int_{\Gamma^{i n}} \int_{\Gamma^{i n} \backslash\{x\}} \nabla_{x} E_{k}(x, y) \cdot\left(\boldsymbol{\Phi}_{i}^{I}(y) \times \boldsymbol{\Phi}_{j}^{I}(x)\right) \mathrm{d} s_{y} \mathrm{~d} s_{x}
\end{aligned}
$$

This shows $A_{12}[i, j]=\beta^{e x} A_{21}[j, i]$, and $A_{13}[i, j]=\beta^{i n} A_{31}[j, i]$ results from $\mathbf{n}^{e x}(x)=$ $-\mathbf{n}^{i n}(x)$ for $x \in \Gamma^{I}$.

Now we go to the last step to consider the a Priori error estimates for (2.2.10). The difference between this section and Section 2.1.4 is that for the interface problem for the Helmholtz equation we use piecewise constant function spaces and piecewise linear function spaces together and for the Maxwell's equations we just use Raviart-Thomas boundary element spaces. So for the Helmholtz equation we need to use (2.1.9) to define Steklov-Poincaré operators to separate piecewise linear function spaces from piecewise constant function spaces. But for the Maxwell's equations, we just use Raviart-Thomas boundary element spaces, so we can repeat all the work in Section 1.3.5 directly. Then we know that $\left\|\boldsymbol{\varphi}^{I}-\boldsymbol{\varphi}_{h}^{I}\right\|_{\mathbf{L}_{\mathbf{t}}^{2}\left(\Gamma^{I}\right)},\left\|\boldsymbol{\sigma}^{e x}-\boldsymbol{\sigma}_{h}^{e x}\right\|_{\mathbf{L}_{\mathbf{t}}^{2}\left(\Gamma^{e x}\right)}$, and $\left\|\boldsymbol{\sigma}^{i n}-\boldsymbol{\sigma}_{h}^{i n}\right\|_{\mathbf{L}_{\mathbf{t}}^{2}\left(\Gamma^{i n}\right)}$ are linear convergence with the lowest order Raviart-Thomas boundary element spaces. We will not repeat all these technical work. 


\subsubsection{Numerical tests}

We consider one example on the boundary of $\Omega^{i n}=(1 / 3,2 / 3)^{3}$ and $\Omega^{e x}=(0,1)^{3} \backslash$ $\overline{\Omega^{i n}}$. An analytical solution used for the test is (1.3.25). In Table 2.2 the first column is the level of meshes, the second column is the number of degrees of freedom, the third, fifth and seventh columns are $\mathbf{L}^{2}$-error for $\boldsymbol{\sigma}_{h}^{e x}, \boldsymbol{\varphi}_{h}^{I}$ and $\boldsymbol{\sigma}_{h}^{i n}$, respectively. The fourth, sixth and eighth columns are the rate of convergence $(s)$ in $O\left(h^{s}\right)$. Fig. 2.2a, 2.2b and 2.2c are the results of $\boldsymbol{\sigma}_{h}^{e x}, \varphi_{h}^{I}$ and $\boldsymbol{\sigma}_{h}^{i n}$, respectively. The calculation of $\mathbf{L}^{2}$-error and the rate of convergence is the same as Section 1.3.6.

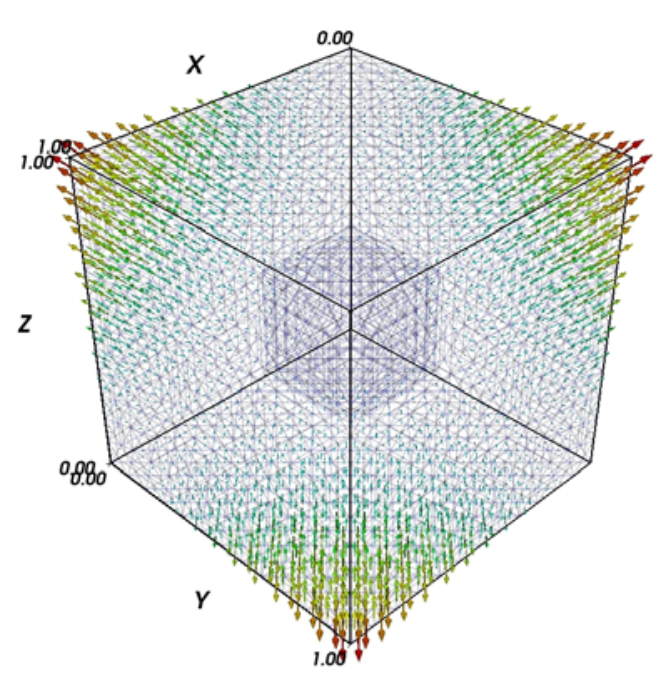

(a) $\sigma^{e x}$, Neumann trace from exterior domain

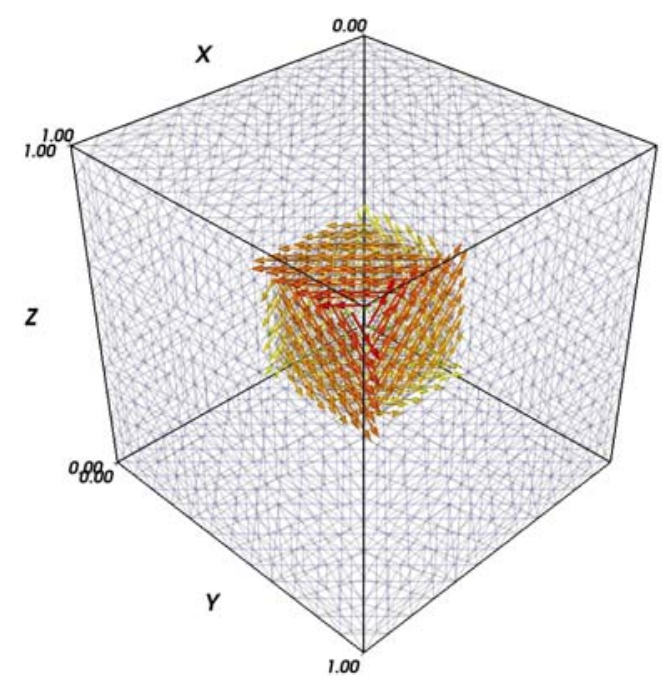

(b) $\varphi^{I}$, Dirichlet trace from exterior domain on interface

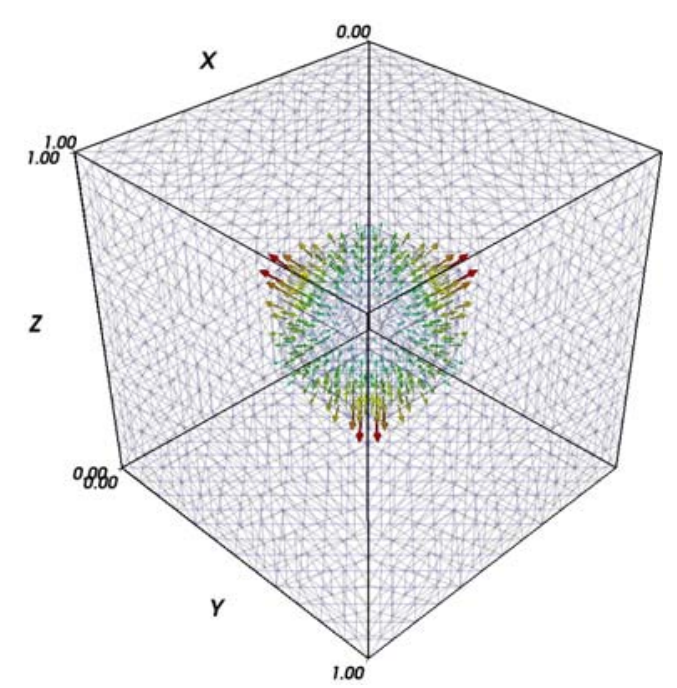

(c) $\sigma^{\text {in }}$, Neumann trace from interior domain

Figure 2.2 Interface problem with Dirichlet boundary condition for Maxwell's equations 


\begin{tabular}{|c|c|c|c|c|c|c|c|}
\hline level & dof & $\left\|\boldsymbol{\sigma}^{e x}-\boldsymbol{\sigma}_{h}^{e x}\right\|_{\mathbf{L}^{2}}$ & $\mathrm{CR}$ & $\left\|\boldsymbol{\varphi}^{I}-\boldsymbol{\varphi}_{h}^{I}\right\|_{\mathbf{L}^{2}}$ & $\mathrm{CR}$ & $\left\|\boldsymbol{\sigma}^{i n}-\boldsymbol{\sigma}_{h}^{i n}\right\|_{\mathbf{L}^{2}}$ & $\mathrm{CR}$ \\
\hline 0 & 432 & 0.17427 & - & 0.04833 & - & 0.01998 & - \\
\hline 1 & 1728 & 0.08608 & 1.0177 & 0.02515 & 0.9426 & 0.01012 & 0.9810 \\
\hline 2 & 6912 & 0.04265 & 1.0131 & 0.01268 & 0.9878 & 0.00499 & 1.0210 \\
\hline
\end{tabular}

Table 2.2 Accuracy of Galerkin-BEMs for interface problem with Dirichlet boundary condition for Maxwell's equations

In the fourth, sixth and eighth columns of Table 2.2, we observe a linear convergence for the lowest order Raviart-Thomas boundary element spaces. Our numerical results match the a Priori error estimates very well. 


\section{Chapter 3}

\section{Boundary Element Methods for Eigenvalue Problems}

\subsection{A Priori error estimates for holomorphic eigenvalue problems}

If we use Galerkin-BEMs to solve a linear eigenvalue problem, the eigenvalue is a parameter in the fundamental solution. So the linear eigenvalue problem is changed to a nonlinear eigenvalue problem. For the study of this nonlinear problem, we follow the $\mathrm{PhD}$ work of Dr. G. Unger [71]. In his thesis he presents a method to compute eigenvalues of the Helmholtz equation and he provides a Priori error estimates for eigenvalues and eigenvectors. His results could be applied to nonlinear operator functions satisfying:

1. Operator functions are holomorphic;

2. The operator defined by an operator function with a fixed value is a Fredholm operator with index zero and the operator can be splitted into an elliptic operator and a compact operator. For example,

$$
H(k)=E+C(k),
$$

where $E$ is an elliptic operator and $C(k)$ is a compact operator.

Definition 5. The operator $E: X \rightarrow X^{\prime}$ is called $X$-elliptic if

$$
\langle E x, x\rangle \geq c\|x\|_{X}^{2} \quad \forall x \in X
$$

where $X$ is a Hilbert space, $X^{\prime}$ is the dual space of $X$ and $\langle\cdot, \cdot\rangle$ is the dual pairing.

In this section we give a brief summary of the work in [71] and we will use these results to check the convergence of our numerical results.

\subsubsection{Basic definitions}

First we define some notations. Let $\Lambda$ denote an open and connected subset of $\mathbb{C}$. Let $\mathcal{L}(X, Y)$ denote a space of bounded linear operators from $X$ to $Y . X$ and $Y$ are Banach 
spaces. Since $X$ and $Y$ are Banach spaces, $\mathcal{L}(X, Y)$ is also a Banach space. If $X$ is a Hilbert space, let $\left\{X^{n}\right\}_{n \in \mathbb{N}}$ denote a sequence of finite dimensional subspaces of $X$ such that $X^{n} \subset X^{n+1}$ and

$$
\lim _{n \rightarrow \infty} \inf _{x^{n} \in X^{n}}\left\|x^{n}-x\right\|_{X}=0 \quad \forall x \in X
$$

Let $P^{n}$ denote a projection from $X$ to $X^{n}$ by the best approximation in $X$.

Definition 6. Let $B$ be a Banach space. A function $h: \Lambda \rightarrow B$ is holomorphic on $\Lambda$ if there exists a function $h^{\prime}: \Lambda \rightarrow B$ such that

$$
\lim _{\epsilon \rightarrow 0}\left\|\frac{h(k+\epsilon)-h(k)}{\epsilon}-h^{\prime}(k)\right\|_{B}=0 \quad \forall k \in \Lambda .
$$

One can show that holomorphic functions are analytic [71, Definition 3.1.1]. In our work, $B$ is a Banach space $\mathcal{L}(X, Y)$, so we call $h$ an operator function. If $h(k)$ is a Fredholm operator for all $k \in \Lambda$, we call $h$ a Fredholm operator function. Then we can define a holomorphic Fredholm operator function $h$. We characterize a holomorphic operator function based on [43, Theorem 3.12] as the following lemma.

Lemma 13. An operator function $H: \Lambda \rightarrow \mathcal{L}(X, Y)$ is holomorphic if the function defined by

$$
k \in \Lambda \rightarrow\langle H(k) x, y\rangle_{Y \times Y^{\prime}}
$$

is holomorphic for all $x \in X$ and $y \in Y^{\prime}$. $Y^{\prime}$ is the dual space of $Y$.

Definition 7. A number $k^{0} \in \Lambda$ is called an eigenvalue of a holomorphic operator function $H$ if there exists a nontrivial solution $x^{0} \in X$ such that

$$
H\left(k^{0}\right) x^{0}=0 .
$$

Every $x^{0}$ satisfying (3.1.3) is called an eigenvector of $H$ corresponding to $k^{0}$.

The definitions of the resolvent $\rho(H)$ and spectrum $\sigma(H)$ are similar to linear eigenvalue problems and could be found in [71, Definition 3.1.5].

In the proof of a Priori error estimates we need to define Jordan chains and Jordan functions for holomorphic operator functions.

Definition 8. Let $H: \Lambda \rightarrow \mathcal{L}(X, Y)$ be a holomorphic operator function. Let $k^{0}$ be an eigenvalue of $H$ with a corresponding eigenvector $x^{0}$. A Jordan chain with order $m$ is a set $\left\{x^{0}, x^{1}, \cdots, x^{m-1}\right\} \subset X$ such that

$$
H\left(k^{0}\right) x^{0}=0 \quad \text { and } \quad \sum_{j=0}^{i} \frac{1}{j !} H^{(j)}\left(k^{0}\right) x^{i-j}=0 \quad \text { for } i=1, \cdots, m-1 .
$$

For holomorphic Fredholm operators all Jordan chains are finite [71, Lemma 3.2.4].

Definition 9. Let $H: \Lambda \rightarrow \mathcal{L}(X, Y)$ be a holomorphic operator function. Let $k^{0}$ be an eigenvalue of $H$ with a corresponding eigenvector $x^{0}$. A Jordan function $u: \Lambda \rightarrow X$ with 
order $m$ is defined in a neighborhood of $k^{0}$ such that $u\left(k^{0}\right)=x^{0}$,

$$
\begin{aligned}
H\left(k^{0}\right) u\left(k^{0}\right)=0 \quad \text { and } \quad & {\left[\frac{\partial^{j}}{\partial k^{j}}(H(k) u(k))\right]_{k=k^{0}}=0 \quad j=1, \cdots, m-1, } \\
& {\left[\frac{\partial^{m}}{\partial k^{m}}(H(k) u(k))\right]_{k=k^{0}} \neq 0 . }
\end{aligned}
$$

Jordan functions can be constructed by Jordan chains [71, Lemma 3.2.13]. From the definitions of Jordan chains and Jordan functions we know that there are different Jordan chains and Jordan functions corresponding to the same eigenvalue and eigenvector. By using order $m$ we can identify eigenvectors to define a canonical system.

Definition 10. Let $H: \Lambda \rightarrow \mathcal{L}(X, Y)$ be a holomorphic Fredholm operator function. Let $k^{0}$ be an eigenvalue of $H$ such that the eigenspace has finite dimension. Let $\left\{x_{1}^{0}, \cdots, x_{J}^{0}\right\}$ be a basis of the eigenspace corresponding to $k^{0}$ and $m\left(H, k^{0}, x_{j}^{0}\right)$ denote the highest order of a Jordan chain corresponding to $\left(k^{0}, x_{j}^{0}\right)$ for $j=1, \cdots, J$. We call $m\left(H, k^{0}, x_{j}^{0}\right)$ the partial multiplicity and the sum of partial multiplicities is called the algebraic multiplicity. A canonical system corresponding to $k^{0}$ is defined by a set of $\left\{x_{1}^{0}, \cdots, x_{J}^{0}\right\}$ ordered by partial multiplicities.

\subsubsection{Convergence}

The eigenvalue problem in our work is to find $k^{0} \in \Lambda$ with a nontrivial solution $x^{0} \in X$ such that

$$
\left(H\left(k^{0}\right) x^{0}, y\right)_{X}=0 \quad \forall y \in X,
$$

where $H: \Lambda \rightarrow \mathcal{L}(X, X)$ is a holomorphic Fredholm operator function and $H$ satisfies (3.1.1). $(\cdot, \cdot)_{X}$ is an inner product of $X$.

Remark 1. The reason to consider $H: \Lambda \rightarrow \mathcal{L}(X, X)$ is to use an inner product and only one function space for this complicated and very technical convergence analysis.

The discrete problem is to find $k^{0, n} \in \Lambda$ and a nontrivial solution $x^{0, n} \in X^{n}$ such that

$$
\left(H\left(k^{0, n}\right) x^{0, n}, y^{n}\right)_{X}=0 \quad \forall y^{n} \in X^{n} .
$$

By the orthogonality from (3.1.5) we have

$$
P^{n} H\left(k^{0, n}\right) x^{0, n}=0 .
$$

Obviously (3.1.5) is equivalent to (3.1.6).

Remark 2. The eigenvalue problem for $P^{n} H(k)$ is to find an eigenvalue $k^{0, n} \in \Lambda$ and an eigenvector $x^{0, n} \in X^{n}$ for (3.1.6).

Lemma 14. Let $H: \Lambda \rightarrow \mathcal{L}(X, X)$ be a holomorphic Fredholm operator function and satisfy (3.1.1). Let $\left\{k^{0, n}\right\}_{n \in \mathbb{N}} \in \Lambda$ be a sequence such that

$$
\lim _{n \rightarrow \infty} k^{0, n}=k^{0} .
$$


Let $\left\{x^{0, n}\right\}_{n \in \mathbb{N}}$ be a sequence for $x^{0, n} \in X^{n}$ and $\left\|x^{0, n}\right\|_{X}=1$ such that

$$
\lim _{n \rightarrow \infty} P^{n} H\left(k^{0, n}\right) x^{0, n}=0 .
$$

Then there exists $x^{0} \in X$ with $\left\|x^{0}\right\|_{X}=1$ such that

$$
\begin{gathered}
H\left(k^{0}\right) x^{0}=0, \\
\lim _{l \rightarrow \infty}\left\|x^{0}-x^{0, n_{l}}\right\|_{X} \rightarrow 0,
\end{gathered}
$$

where $\left\{x^{0, n_{l}}\right\}_{l \in \mathbb{N}}$ is a subsequence of $\left\{x^{0, n}\right\}_{n \in \mathbb{N}}$.

Proof. This Lemma is given in [71, Lemma 4.2.1] which follows [31].

Obviously the last lemma does not give the existence of a converging sequence of eigenvalues of (3.1.6). We give a theorem from [71, Theorem 4.2.3] which could be used for the convergence of Galerkin-BEMs for the nonlinear problem (3.1.6).

Theorem 6. Let $H: \Lambda \rightarrow \mathcal{L}(X, X)$ be a holomorphic Fredholm operator function and satisfy (3.1.1). Let $k^{0, n}$ be an eigenvalue of (3.1.6) with a corresponding eigenvector $x^{0, n} \in X^{n}$. Then we have

$$
\lim _{n \rightarrow \infty} k^{0, n}=k^{0} \quad \text { and } \quad \lim _{n \rightarrow \infty} x^{0, n}=x^{0},
$$

$k^{0}$ is an eigenvalue of $H$ with a corresponding eigenvector $x^{0} \in X$.

Proof. The proof follows the part (i) in [71, Theorem 4.2.3] by Lemma 14.

\subsubsection{A Priori error estimates}

The work for a Priori error estimates in [71] follows [41] and [42]. The basic idea is to construct the equivalent eigenvalue problems $M$ for $H$ and $M^{n}$ for $P^{n} H$ respectively. $M$ and $M^{n}$ are matrix functions. Then the work is changed to do a Priori error estimates for $M^{n}$. Lemma 15 is from [71, Lemma 4.3.1] and it is the basis to construct equivalent problems.

Lemma 15. Let $X$ and $Z$ be Banach spaces. We define five holomorphic operator functions, $H: \Lambda \rightarrow \mathcal{L}(X, X), R: \Lambda \rightarrow \mathcal{L}(X, X), C: \Lambda \rightarrow \mathcal{L}(X, Z), D: \Lambda \rightarrow \mathcal{L}(Z, X)$, and $M: \Lambda \rightarrow \mathcal{L}(Z, Z)$, such that

$$
\begin{aligned}
H(k) & =R(k)\left(I_{X}-D(k) C(k)\right), \\
M(k) & =I_{Z}-C(k) D(k),
\end{aligned}
$$

for all $k \in \Lambda$. We assume that $H$ is a holomorphic Fredholm operator function. We assume that $\rho(H)$ is not empty and $\Lambda \subset \rho(R)$. Then we have the following relations for $k^{0} \in \sigma(H), x^{0} \in X$ and $z^{0} \in Z$.

1. If $x^{0} \neq 0$ such that $H\left(k^{0}\right) x^{0}=0$, we have $C\left(k^{0}\right) x^{0} \neq 0$ such that $M\left(k^{0}\right) C\left(k^{0}\right) x^{0}=$ 0 and $m\left(H, k^{0}, x^{0}\right)=m\left(M, k^{0}, C\left(k^{0}\right) x^{0}\right)$. 
2. If $z^{0} \neq 0$ such that $M\left(k^{0}\right) z^{0}=0$, we have $D\left(k^{0}\right) z^{0} \neq 0$ such that $H\left(k^{0}\right) D\left(k^{0}\right) z^{0}=$ 0 and $m\left(M, k^{0}, z^{0}\right)=m\left(H, k^{0}, D\left(k^{0}\right) z^{0}\right)$.

3. If $u(k)$ is a Jordan function of order $m$ for $H$ to $k^{0}, C(k) u(k)$ is a Jordan function of order $m^{\prime} \geq m$ for $M$ to $k^{0}$.

4. If $v(k)$ is a Jordan function of order $m^{\prime}$ for $M$ to $k^{0}, D(k) v(k)$ is a Jordan function of order $m \geq m^{\prime}$ for $H$ to $k^{0}$.

5. A canonical system of $H$ to $k^{0}$ could be changed to a canonical system of $M$ to $k^{0}$ by a mapping $C\left(k^{0}\right)$ with the same partial and algebraic multiplicities.

Proof. See [71, Lemma 4.3.1].

From Lemma 15, the idea is clear to build an equivalent eigenvalue problem $M$. Let $Z=\mathbb{C}^{J}$ and $J$ is the dimension of the eigenspace of $k^{0}$. Then $M$ is a matrix function from $\mathbb{C}^{J}$ to $\mathbb{C}^{J}$. The next step is to build $R, C$ and $D$ which satisfy the requirements in Lemma 15. The basic idea is to define a finite dimensional operator by using Jordan functions based on Jordan chains. Jordan chains are corresponding to two canonical systems of an eigenvalue $k^{0}$ of $H$ and an eigenvalue $\overline{k^{0}}$ of $H^{*}$ respectively. $H^{*}$ is the adjoint operator of $H$. $k^{0}$ is an eigenvalue of $H$ if and only if $\overline{k^{0}}$ is an eigenvalue of $H^{*}$ and the partial and algebraic multiplicities are the same from [71, Lemma 3.3.4].

Let $H: \Lambda \rightarrow \mathcal{L}(X, X)$ be a holomorphic Fredholm operator function. The reason to use $\mathcal{L}(X, X)$ is the same as Remark 2. Let $\left\{x_{1}^{0}, \cdots, x_{J}^{0}\right\}$ be a canonical system of an eigenvalue $k^{0}$ of $H$ and we simplify the notation for partial multiplicities $m\left(H, k^{0}, x_{j}^{0}\right)$ to $m_{j}$. Let $\left\{x_{j}^{0}, \cdots, x_{j}^{m_{j}-1}\right\}$ be a Jordan chain with order $m_{j}$ corresponding to $x_{j}^{0}$ for $j=1, \cdots, J$. According [71, Lemma 3.2.13] we can define a Jordan function with order $m_{j}$ by $\left\{x_{j}^{0}, \cdots, x_{j}^{m_{j}-1}\right\}$,

$$
u_{j}(k)=\sum_{i=0}^{m_{j}-1}\left(k-k^{0}\right)^{i} x_{j}^{i} \quad j=1, \cdots, J .
$$

By the definition of a Jordan function with order $m_{j}$, we have

$$
\left[\frac{\partial^{m_{j}}}{\partial k^{m_{j}}}\left(H(k) u_{j}(k)\right)\right]_{k=k^{0}} \neq 0 .
$$

We define holomorphic functions as

$$
\widehat{u}_{j}(k):=\left\{\begin{array}{ll}
\left(k-k^{0}\right)^{-m_{j}} H(k) u_{j}(k) & k \neq k^{0} \\
\frac{1}{m_{j} !}\left[\frac{\partial^{m_{j}}}{\partial k^{m_{j}}}\left(H(k) u_{j}(k)\right)\right]_{k=k^{0}} & k=k^{0}
\end{array} \quad j=1, \cdots, J .\right.
$$

We can repeat this for the adjoint operator $H^{*}$ and define

$$
\widehat{v}_{j}(k):=\left\{\begin{array}{ll}
\left(k-\overline{k^{0}}\right)^{-m_{j}} H^{*}(k) v_{j}(k) & k \neq \overline{k^{0}} \\
\frac{1}{m_{j} !}\left[\frac{\partial^{m_{j}}}{\partial k^{m_{j}}}\left(H^{*}(k) v_{j}(k)\right)\right]_{k=\overline{k^{0}}} & k=\overline{k^{0}}
\end{array} \quad j=1, \cdots, J,\right.
$$


where the definition $v_{j}$ is similar with $u_{j}$.

Then we define an operator function

$$
K(k) x:=\sum_{j=1}^{J}\left(x, \widehat{v}_{j}(\bar{k})\right)_{X} \widehat{u}_{j}(k) .
$$

$K$ is holomorphic and the proof see [71, Section 4.3]. $R$ is defined by

$$
R(k):=H(k)+K(k) .
$$

Lemma 4.3.3 in [71] proves that there exists a neighborhood $\Lambda$ of an eigenvalue $k^{0}$ which satisfies the condition $\Lambda \subset \rho(R)$ in Lemma 15. By using (3.1.8) in (3.1.9) we get

$$
\begin{aligned}
H(k) x & =(R(k)-K(k)) x=R(k)\left(I-R(k)^{-1} K(k)\right) x \\
& =R(k)\left(x-\sum_{j=1}^{J}\left(x, \widehat{v}_{j}(\bar{k})\right)_{X} R(k)^{-1} \widehat{u}_{j}(k)\right) .
\end{aligned}
$$

From (3.1.10) we can define $C$ and $D$ as the following.

$$
C(k): x \in X \rightarrow a \in \mathbb{C}^{J},
$$

with $a_{i}=\left(x, \widehat{v}_{i}(\bar{k})\right)_{X}$ for $i=1, \cdots, J$.

$$
D(k): a \in \mathbb{C}^{J} \rightarrow x \in X,
$$

with $x=\sum_{j=1}^{J} a_{j} R(k)^{-1} \widehat{u}_{j}(k)$. According (3.1.7b), (3.1.11) and (3.1.12), we define $M(k)=I-C(k) D(k)$ as

$$
M(k): a \in \mathbb{C}^{J} \rightarrow b \in \mathbb{C}^{J},
$$

with $b_{i}=a_{i}-\sum_{j=1}^{J} a_{j}\left(R(k)^{-1} \widehat{u}_{j}(k), \widehat{v}_{i}(\bar{k})\right)_{X}$. More detial see [71, Section 4.3].

We continue to define corresponding operators for a discretization $X^{n}$. The definition of $P^{n}$ is given in Section 3.1.1. We define

$$
\begin{array}{ll}
H^{n}(k): H^{n}(k) x=P^{n} H(k) x & \forall x \in X^{n}, \\
K^{n}(k): K^{n}(k) x=P^{n} K(k) x & \forall x \in X^{n}, \\
R^{n}(k):=H^{n}(k)+K^{n}(k): X^{n} \rightarrow X^{n} .
\end{array}
$$

Lemma 4.3.3 in [71] also proves that $R^{n}$ satisfies the conditions in Lemma 15. We have

$$
\begin{aligned}
H^{n}(k) x & =\left(R^{n}(k)-K^{n}(k)\right) x \\
& =R^{n}(k)\left(I-R^{n}(k)^{-1} K^{n}(k)\right) x \\
& =R^{n}(k)\left(x-\sum_{j=1}^{J}\left(x, \widehat{v}_{j}(\bar{k})\right)_{X}\left(R^{n}(k)\right)^{-1} P^{n} \widehat{u}_{j}(k)\right) \quad \forall x \in X^{n} .
\end{aligned}
$$


The definitions of $C^{n}$ and $D^{n}$ are

$$
C^{n}(k): x \in X^{n} \rightarrow a \in \mathbb{C}^{J}
$$

with $a_{i}=\left(x, \widehat{v}_{i}(\bar{k})\right)_{X}$ for $i=1, \cdots, J$ and

$$
D^{n}(k): a \in \mathbb{C}^{J} \rightarrow x \in X^{n}
$$

with $x=\sum_{j=1}^{J} a_{j}\left(R^{n}(k)\right)^{-1} P^{n} \widehat{u}_{j}(k)$. According (3.1.15) and (3.1.16) we define $M^{n}$ as

$$
M^{n}(k): a \in \mathbb{C}^{J} \rightarrow b \in \mathbb{C}^{J},
$$

with $b_{i}=a_{i}-\sum_{j=1}^{J} a_{j}\left(R(k)^{-1} P^{n} \widehat{u}_{j}(k), \widehat{v}_{i}(\bar{k})\right)_{X}$. More detial see [71, Section 4.3].

According Lemma 15, $M$ is an equivalent eigenvalue problem of $H$ and $M^{n}$ is an equivalent eigenvalue problem of $H^{n}$. For a Priori error estimates of $M^{n}$ the following results are given in [71, Chapter 4].

Lemma 16. Let $\Lambda$ satisfy the condition $\Lambda \subset \rho(R)$ in Lemma 15. Let $\Lambda_{c}$ be a compact subset of $\Lambda$. c depends on $\Lambda_{c}$ and $c>0$. There exist a $N \in \mathbb{N}$ such that for all $n>N$

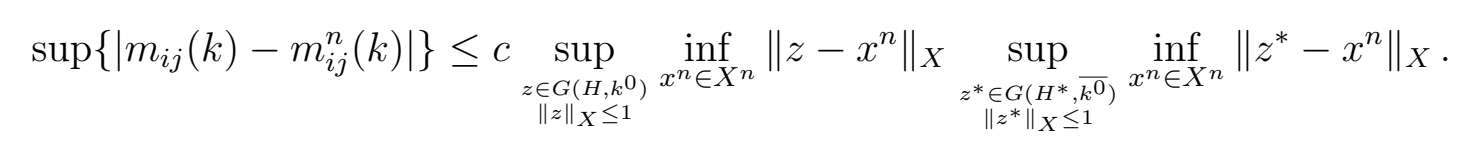

for $k \in \Lambda_{c}$ and $G\left(H, k^{0}\right)$ and $G\left(H^{*}, \overline{k^{0}}\right)$ are the generalized eigenspaces corresponding to $H$ and $k^{0}$, and $H^{*}$ and $\overline{k^{0}}$, respectively.

Proof. See [71, Lemma 4.3.5].

Lemma 16 is the key tool to do a Priori error estimates for eigenvalues and eigenvectors. From this lemma we can see the reasons to use equivalent eigenvalue problems. Since $M$ and $M^{n}$ are finite dimensional operators, we just need to consider $\left|m_{i j}(k)-m_{i j}^{n}(k)\right|$ and this is much easier. From (3.1.13) and (3.1.17) we know that we can use the properties of $R$ and $R^{n}$ in the proof of Lemma 16. $R$ and $R^{n}$ are invertible in $\Lambda$ and the inverse of $R^{n}$ is uniformly bounded. This is the other reason to use $M$ and $M^{n}$.

We define

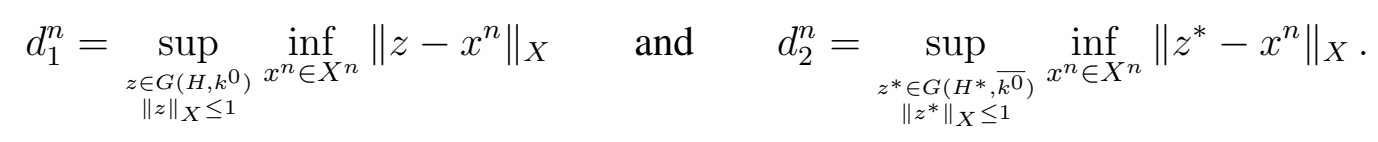

Theorem 7 gives a Priori error estimate for eigenvalues by using the result from Lemma 16. In our numerical examples we will use this theorem to check the convergence for eigenvalues. In our cases we don't consider the convergence of eigenvectors.

Theorem 7. Let $H: \Lambda \rightarrow \mathcal{L}(X, X)$ be a holomorphic Fredholm operator function and satisfy (3.1.1). Let $\Lambda_{c}$ be a compact subset of $\Lambda . \partial \Lambda_{c} \subset \rho(H)$ and $\Lambda_{c} \bigcap \sigma(H)=\left\{k^{0}\right\}$. There exist a $N \in \mathbb{N}$ such that

$$
\sigma\left(P^{n} H\right) \bigcap \Lambda_{c} \neq \emptyset \quad \forall n>N .
$$


Let $k^{0, n}$ be an eigenvalue of $P^{n} H$ and $k^{0, n} \in \sigma\left(P^{n} H\right) \bigcap \Lambda_{c}$. There exist a constant $c>0$ such that

$$
\left|k^{0, n}-k^{0}\right| \leq c\left(d_{1}^{n} d_{2}^{n}\right)^{1 / \varkappa} \quad \forall n>N,
$$

where $\varkappa$ is the maximal length of Jordan chains of $k^{0}$.

Proof. See Theorem 4.3.6 in [71]

A Priori error estimate for eigenvectors is also given in [71, Theorem 4.3.7].

\subsection{The Helmholtz case}

From Section 1.1 we know that if we want to calculate $\lambda$ and e together in (1.1.20), this is an eigenvalue problem. In this section we study the eigenvalue problem for the Helmholtz equation with a homogeneous Dirichlet boundary condition. Find $\lambda$ with a nontrivial solution $u$ such that

$$
\begin{aligned}
-\Delta u(x) & =\lambda u(x) & & \forall x \in \Omega, \\
u(x) & =0 & & \forall x \in \Gamma,
\end{aligned}
$$

where $\lambda$ is a positive real number, $\Omega$ is a bounded domain and $\Gamma:=\partial \Omega$.

\subsubsection{Nonlinear solution method for eigenvalue problem}

We define $\sigma:=\gamma_{1} u$. From the boundary integral equation (1.2.12) we have

$$
V_{k}(\sigma)=\left(\frac{1}{2} I+K_{k}\right) f
$$

where $f$ is the Dirichlet given data and $k=\sqrt{\lambda}$. The homogeneous Dirichlet boundary condition (3.2.1b) yields $f=0$, so the boundary integral equation is

$$
V_{k}(\sigma)=0
$$

Obviously, $k$ is a parameter in the fundamental solution $E_{k}(\cdot, \cdot)$, so this is a nonlinear eigenvalue problem.

The variation formulation is to find $k \in \mathbb{R}^{+}$with a nontrivial solution $\sigma \in H^{-1 / 2}(\Gamma)$ such that

$$
\left\langle V_{k}(\sigma), \chi\right\rangle_{\Gamma}=0
$$

for all $\chi \in H^{-1 / 2}(\Gamma)$.

The discretization of (3.2.3) is to find $k_{h} \in \mathbb{R}^{+}$with a nontrivial solution $\sigma_{h} \in S_{h}^{0}\left(\Gamma_{h}\right)$ such that

$$
\left\langle V_{k_{h}}\left(\sigma_{h}\right), \chi_{h}\right\rangle_{\Gamma}=0
$$


for all $\chi_{h} \in S_{h}^{0}\left(\Gamma_{h}\right)$.

Let $\left\{\Phi_{i}\right\}_{i=1}^{N}$ be a basis of $S_{h}^{0}\left(\Gamma_{h}\right)$. We use $\sigma_{h}=\sum_{j=1}^{N} \xi_{j} \Phi_{j}$ in (3.2.4) and get a nonlinear system of equations

$$
A\left(k_{h}\right) \xi=0
$$

where

$$
A\left(k_{h}\right)[i, j]=\operatorname{Re}\left(\int_{\Omega_{i}} \int_{\Omega_{j}} \frac{\mathrm{e}^{\mathrm{i} k_{h}|x-y|}}{4 \pi|x-y|} \mathrm{d} s_{y} \mathrm{~d} s_{x}\right) .
$$

\section{Iterative methods for nonlinear solution method}

We use the Newton method to solve (3.2.5). To avoid the trivial solution of (3.2.5) we need to do the normalization for $\xi$ by $\|\xi\|_{l^{2}}^{2}=1$. The nonlinear system of equations is

$$
\begin{aligned}
& A\left(k_{h}\right) \xi=0, \\
& \|\xi\|_{l^{2}}^{2}-1=0 .
\end{aligned}
$$

The Frechet derivative is derived by

$$
\begin{aligned}
\lim _{\epsilon \rightarrow 0} \frac{A\left(k_{h}+\epsilon k_{h}^{\prime}\right)\left(\xi+\epsilon \xi^{\prime}\right)-A\left(k_{h}\right)(\xi)}{\epsilon} & =A\left(k_{h}\right) \xi^{\prime}+\left(\lim _{\epsilon \rightarrow 0} \frac{A\left(k_{h}+\epsilon k_{h}^{\prime}\right)-A\left(k_{h}\right)}{\epsilon}\right) \xi \\
& =A\left(k_{h}\right) \xi^{\prime}+k_{h}^{\prime} C\left(k_{h}\right) \xi
\end{aligned}
$$

where

$$
\begin{gathered}
C\left(k_{h}\right)[i, j]=\operatorname{Re}\left(\frac{1}{4 \pi} \int_{\Omega_{i}} \int_{\Omega_{j}} \frac{\mathrm{i}^{\mathrm{i} k_{h}|x-y|}}{4 \pi} \mathrm{d} s_{y} \mathrm{~d} s_{x}\right) . \\
\lim _{h \rightarrow 0} \frac{\left\|\xi+h \xi^{\prime}\right\|_{l^{2}}^{2}-\|\xi\|_{l^{2}}^{2}}{h}=2\left(\xi, \xi^{\prime}\right)_{l^{2}} .
\end{gathered}
$$

The Newton method is defined by

$$
\begin{aligned}
& A\left(k_{h}^{n}\right)\left(\xi^{n+1}-\xi^{n}\right)+\left(k_{h}^{n+1}-k_{h}^{n}\right) C\left(k_{h}^{n}\right) \xi^{n}+A\left(k_{h}^{n}\right) \xi^{n}=0, \\
& 2\left(\xi^{n}, \xi^{n+1}-\xi^{n}\right) l_{l^{2}}+\left\|\xi^{n}\right\|_{l^{2}}^{2}-1=0,
\end{aligned}
$$

and the iteration step is to find $\left(k_{h}^{n+1}, \xi^{n+1}\right)$ with the given data $\left(k_{h}^{n}, \xi^{n}\right)$ by solving

$$
\begin{aligned}
& A\left(k_{h}^{n}\right) \xi^{n+1}+k_{h}^{n+1} C\left(k_{h}^{n}\right) \xi^{n}=k_{h}^{n} C\left(k_{h}^{n}\right) \xi^{n}, \\
& 2\left(\xi^{n}, \xi^{n+1}\right) l^{2}=\left\|\xi^{n}\right\|_{l^{2}}^{2}+1 .
\end{aligned}
$$

\subsubsection{A Priori error estimates}

If we want to give a Priori error estimates for eigenvalue values of (3.2.3) and (3.2.4) by Theorem 7, we need to check that $V_{k}$ is a holomorphic Fredholm operator function and satisfies (3.1.1). In our work we consider electromagnetic waves in a dielectric medium, so $k$ is a real positive number. We can extend $k$ to be a complex number and the results 
are the same. We change the notation from $V_{k}$ to $V(k)$. First we have

$$
V(k)=V(0)+V(k)-V(0),
$$

where $V(0)$ is an elliptic operator from $H^{-1 / 2}(\Gamma)$ to $H^{1 / 2}(\Gamma)$ and $V(k)-V(0)$ is a compact operator. So $V(k)$ satisfies 3.1.1. Next by using the Gårding inequality in Lemma 3 and the Fredholm alternative we know that $V(k)$ is a Fredholm operator with index zero for all $k \in \mathbb{C}$. Then we give a lemma to show that $V(k)$ is holomorphic following [71, Lemma 5.1.1].

Lemma 17. The operator function $V(k)$ is holomorphic.

Proof. The proof uses Lemma 13. We define a function as

$$
\begin{gathered}
f_{v, w}(k):=\langle(V(k))(v), w\rangle_{\Gamma} \quad \forall v, w \in H^{-1 / 2}(\Gamma) . \\
f_{v, w}(k)=\int_{\Gamma} \int_{\Gamma} \frac{\mathrm{e}^{\mathrm{i} k|x-y|}}{4 \pi|x-y|} v(y) w(x) \mathrm{d} s_{y} \mathrm{~d} s_{x}
\end{gathered}
$$

By using the Taylor expansion we have

$$
\mathrm{e}^{\mathrm{i} k|x-y|}=\sum_{n=0}^{\infty} \frac{(\mathrm{i} k|x-y|)^{n}}{n !} .
$$

We use $\mathrm{e}^{\mathrm{i} k|x-y|}$ in $f_{v, w}(k)$ and get

$$
f_{v, w}(k)=\sum_{n=0}^{\infty}\left(\int_{\Gamma} \int_{\Gamma} \frac{\mathrm{i}^{n}|x-y|^{n-1}}{4 \pi n !} v(y) w(x) \mathrm{d} s_{y} \mathrm{~d} s_{x}\right) k^{n} .
$$

So $f_{v, w}(k)$ is a power series in $k$ and the highest order singular integral in the coefficients is a weakly singular integral for $n=0$ which is bounded. So $f_{v, w}(k)$ is a holomorphic function for $k \in \mathbb{C}$. The proof is done.

By Theorem 1 we have

$$
V(k): \mathbb{C} \rightarrow \mathcal{L}\left(H^{-1 / 2}(\Gamma), H^{1 / 2}(\Gamma)\right) .
$$

$V(k)$ does not satisfy the requirement $H(k): \Lambda \rightarrow \mathcal{L}(X, X)$ in Theorem 7. An additional operator $I: H^{1 / 2}(\Gamma) \rightarrow H^{-1 / 2}(\Gamma)$ is defined by [71, (5.9)] and from [71, Theorem 5.1.3] we know that $I V(k): \mathbb{C} \rightarrow \mathcal{L}\left(H^{-1 / 2}(\Gamma), H^{-1 / 2}(\Gamma)\right)$ has the same properties with $V(k)$. Then we can use Theorem 6 for $I V(k)$ with $X=H^{-1 / 2}(\Gamma)$ and by the results of $I V(k)$ we get the convergence of (3.2.4).

Theorem 8. Let $k_{h} \in \mathbb{C}$ be an eigenvalue of (3.2.4) with a corresponding eigenvector $\sigma_{h} \in S_{h}^{0}\left(\Gamma_{h}\right)$. Then there exist $k$ and $\sigma$ such that

$$
\lim _{h \rightarrow 0} k_{h}=k \quad \text { and } \quad \lim _{h \rightarrow 0} \sigma_{h}=\sigma,
$$

$k$ is an eigenvalue of $V(k)$ with a corresponding eigenvector $\sigma \in H^{-1 / 2}(\Gamma)$. 
Proof. See [71, Theorem 5.3.1].

It is the same to use Theorem 7 for $I V(k)$ and by the results of $I V(k)$ with (1.2.22a) we get a Priori error estimates of eigenvalues of (3.2.4).

Theorem 9. Let $k$ be an eigenvalue of $V(k)$. There exists a $\delta$ such that $k$ is the only eigenvalue in $U_{\delta}(k)$. Let $\left\{t^{1}, \cdots, t^{n}\right\}$ be an orthonormal basis of the generalized eigenspace $G(V(k), k)$. Assume that $t^{i} \in H_{p w}^{s}(\Gamma)$ for $s \in[0,1]$. There exists a $h^{0}>0$ such that for all $h<h^{0}$

$$
\left|k_{h}-k\right| \leq c\left(h^{2 s+1}\right)^{1 / \varkappa(V, k)} \sum_{i=1}^{n}\left|t^{i}\right|_{H_{p w}^{s}(\Gamma)},
$$

where $k_{h}$ is the eigenvalue of (3.2.4) and $k_{h} \in U_{\delta}(k)$.

Proof. See Theorem 5.3.2 and Corollary 5.3.3 in [71].

\subsubsection{Numerical tests}

We consider the first and second eigenvalues of the Laplace eigenvalue problem with corresponding eigenvectors on the boundary of a unit cube $(0,1)^{3}$. An analytical solution used for the test is

$$
u(x)=\sin \left(\pi m_{1} x_{1}\right) \sin \left(\pi m_{2} x_{2}\right) \sin \left(\pi m_{3} x_{3}\right) .
$$

where $m_{1}, m_{2}, m_{3}$ are nonzero integers. The eigenvalue $\lambda$ is given by $\sqrt{\lambda}=k=$ $\pi \sqrt{m_{1}^{2}+m_{2}^{2}+m_{3}^{2}}$. In Tables 3.1 and 3.2 the first column is the level of meshes and the second column is the number of degrees of freedom. In Table 3.1 the third column is the numerical results for the first eigenvalue and the fourth column is the rate of convergence $(s)$ in $O\left(h^{2 s+1}\right) . \varkappa=1$ from [67, Lemma 5.3]. In Table 3.2 the third, fifth and seventh columns are the numerical results for the second eigenvalue and the fourth, sixth and eighth columns are the rate of convergence $(s)$ in $O\left(h^{2 s+1}\right)$. Fig. 3.1a is the numerical result of an eigenvector corresponding to the first eigenvalue and Fig. 3.1b - 3.1d are the numerical results of eigenvectors corresponding to the second eigenvalue. In the fourth column in Table 3.1 and the fourth, sixth and eighth columns in Table 3.2 we observe a cubic convergence approximately, since we use piecewise constant function spaces.

\begin{tabular}{|c|c|c|c|}
\hline \multicolumn{2}{|c|}{} & \multicolumn{2}{c|}{$(1,1,1)$} \\
\hline level & dof & $k_{h}$ & $\mathrm{CR}_{k}$ \\
\hline 0 & 24 & 5.04298 & - \\
\hline 1 & 96 & 5.42173 & 4.3404 \\
\hline 2 & 384 & 5.43908 & 3.0860 \\
\hline 3 & 1536 & 5.44116 & 3.2624 \\
\hline$\infty$ & & 5.44140 & \\
\hline
\end{tabular}

Table 3.1 Convergence of the first eigenvalue of the Laplace eigenvalue problem with homogeneous Dirichlet boundary condition 
The formula for the rate of convergence $(s)$ of eigenvalues is given by

$$
\mathrm{CR}_{k}=\log _{2} \frac{\left|k_{h}^{n-1}-k\right|}{\left|k_{h}^{n}-k\right|},
$$

where $k_{h}^{n}$ is the eigenvalue calculated by a mesh of a level $n$.

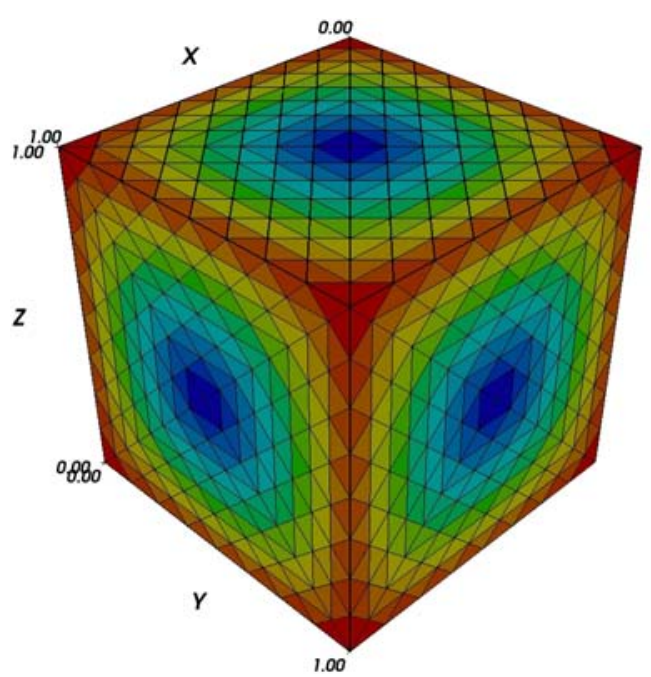

(a) $\sigma_{h}$, Neumann trace of the first eigenvector

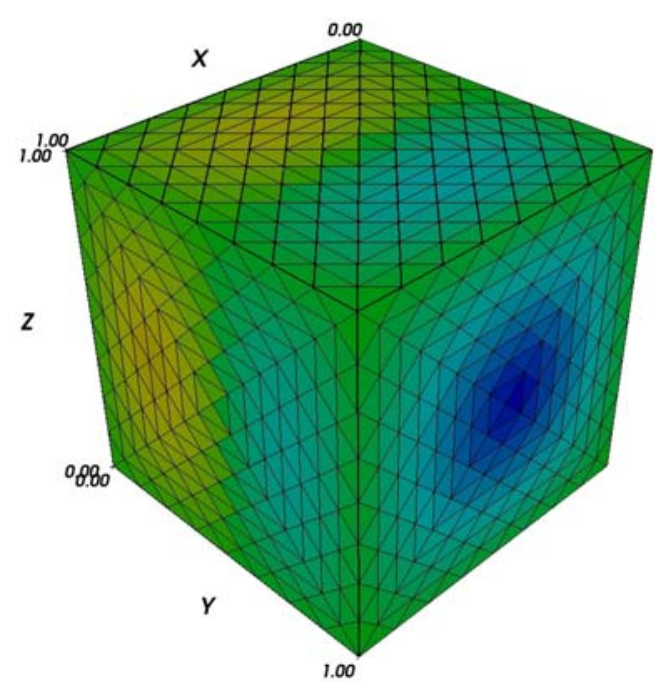

(c) $\sigma_{h}$, Neumann trace of one second eigenvector

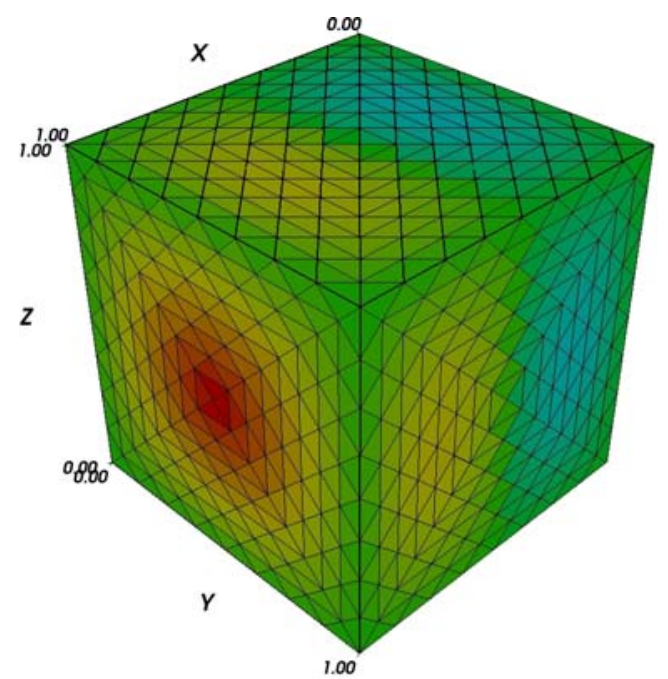

(b) $\sigma_{h}$, Neumann trace of one second eigenvector

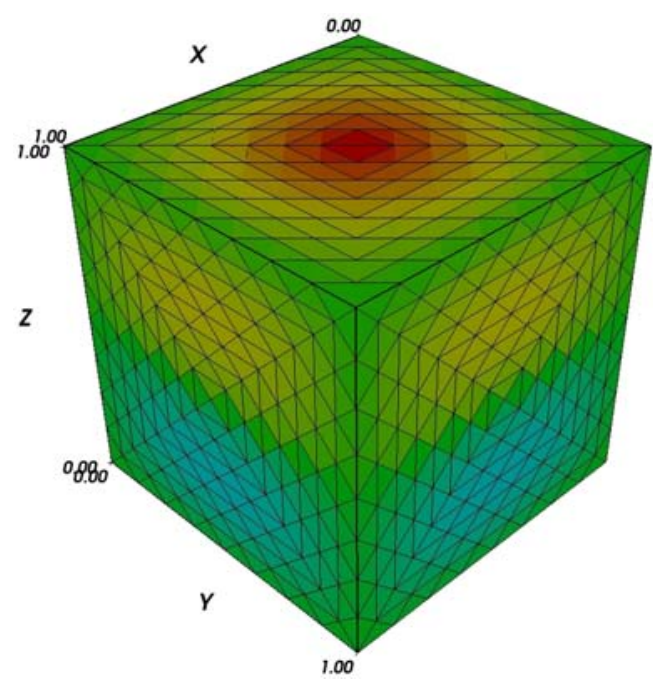

(d) $\sigma_{h}$, Neumann trace of one second eigenvector

Figure 3.1 First eigenvector and second eigenvector of the Laplace eigenvalue problem with homogeneous Dirichlet boundary condition 


\begin{tabular}{|c|c|c|c|c|c|c|c|}
\hline \multicolumn{2}{|c|}{} & \multicolumn{2}{c|}{$(2,1,1)$} & \multicolumn{2}{c|}{$(1,2,1)$} & \multicolumn{2}{c|}{$(1,1,2)$} \\
\hline level & dof & $k_{h}$ & $\mathrm{CR}_{k}$ & $k_{h}$ & $\mathrm{CR}_{k}$ & $k_{h}$ & $\mathrm{CR}_{k}$ \\
\hline 0 & 24 & 7.182768265 & - & 7.182768275 & - & 7.182761379 & - \\
\hline 1 & 96 & 7.619649988 & 2.7602 & 7.619649309 & 2.7602 & 7.619649019 & 2.7602 \\
\hline 2 & 384 & 7.688713513 & 3.5220 & 7.688713646 & 3.5220 & 7.688713542 & 3.5220 \\
\hline 3 & 1536 & 7.694600008 & 3.2360 & 7.694601568 & 3.2392 & 7.694602375 & 3.2409 \\
\hline$\infty$ & & 7.695298981 & & 7.695298981 & & 7.695298981 & \\
\hline
\end{tabular}

Table 3.2 Convergence of the second eigenvalue of the Laplace eigenvalue problem with homogeneous Dirichlet boundary condition

\subsection{The Maxwell case}

In this section we study the eigenvalue problem for the Maxwell's equations. Find $\lambda$ with a nontrivial solution $\mathbf{u}$ such that

$$
\begin{aligned}
\nabla \times \nabla \times \mathbf{u}(x)-\lambda \mathbf{u}(x) & =0 & & \forall x \in \Omega, \\
\nabla \cdot \mathbf{u}(x) & =0 & & \forall x \in \Omega, \\
\gamma_{\mathbf{t}}(\mathbf{u})(x) & =0 & & \forall x \in \Gamma,
\end{aligned}
$$

where $\lambda$ is a real positive number, $\Omega$ is a bounded domain and $\Gamma:=\partial \Omega$.

\subsubsection{Nonlinear solution method for eigenvalue problem}

We define $\boldsymbol{\sigma}:=\gamma_{\mathbf{t}}(\mathbf{u})$. From the boundary integral equation (1.3.15) we get

$$
\mathbf{S}_{k}(\boldsymbol{\sigma})=\left(\frac{1}{2} I-\mathbf{C}_{k}\right) \mathbf{f}
$$

where $\mathbf{f}$ is the Dirichlet given data and $k=\sqrt{\lambda}$. From (3.3.1c) we know $\mathbf{f}=0$, so the boundary integral equations is

$$
\mathbf{S}_{k}(\boldsymbol{\sigma})=0 .
$$

The variational formulation is to find $k$ with a nontrivial solution $\boldsymbol{\sigma} \in \mathbf{W}^{-1 / 2}(\Gamma)$ such that

$$
\left\langle\mathbf{S}_{k}(\boldsymbol{\sigma}), \boldsymbol{\chi}\right\rangle_{\tau, \Gamma}=0
$$

for all $\chi \in \mathbf{W}^{-1 / 2}(\Gamma)$.

The discretization of (3.3.3) is to find $k_{h}$ with a nontrivial solution $\boldsymbol{\sigma}_{h} \in \mathbf{W}_{h}^{-1 / 2}\left(\Gamma_{h}\right)$ such that

$$
\left\langle\mathbf{S}_{k_{h}}\left(\boldsymbol{\sigma}_{h}\right), \chi_{h}\right\rangle_{\tau, \Gamma}=0
$$

for all $\chi_{h} \in \mathbf{W}_{h}^{-1 / 2}\left(\Gamma_{h}\right)$.

Let $\left\{\boldsymbol{\Phi}_{i}\right\}_{i=1}^{N}$ be a basis of $\mathbf{W}_{h}^{-1 / 2}\left(\Gamma_{h}\right)$. We use $\boldsymbol{\sigma}_{h}=\sum_{j=1}^{N} \xi_{j} \boldsymbol{\Phi}_{j}$ in (3.3.4) and get a 
nonlinear equation system,

$$
\begin{aligned}
A\left(k_{h}\right) \xi=0, & \\
A\left(k_{h}\right)[i, j]= & \operatorname{Re}\left(-k_{h} \int_{\Gamma} \int_{\Gamma \backslash\{x\}} \boldsymbol{\Phi}_{j}(y) \cdot \boldsymbol{\Phi}_{i}(x) \frac{\mathrm{e}^{\mathrm{i} k_{h}|x-y|}}{4 \pi|x-y|} \mathrm{d} s_{y} \mathrm{~d} s_{x}\right. \\
& \left.+\frac{1}{k_{h}} \int_{\Gamma} \int_{\Gamma \backslash\{x\}} \operatorname{div}_{\Gamma}\left(\boldsymbol{\Phi}_{j}\right)(y) \operatorname{div}_{\Gamma}\left(\boldsymbol{\Phi}_{i}\right)(x) \frac{\mathrm{e}^{\mathrm{i} k_{h}|x-y|}}{4 \pi|x-y|} \mathrm{d} s_{y} \mathrm{~d} s_{x}\right) .
\end{aligned}
$$

\section{Iterative methods for the nonlinear solution method}

We use the Newton method to solve (3.3.5). To avoid a trivial solution of (3.3.5) we include the normalization for $\xi$ as $\|\xi\|_{l^{2}}^{2}=1$. The nonlinear equation system is

$$
\begin{aligned}
& A\left(k_{h}\right) \xi=0, \\
& \|\xi\|_{l^{2}}^{2}-1=0 .
\end{aligned}
$$

The Frechet derivative is

$$
\begin{aligned}
\lim _{\epsilon \rightarrow 0} \frac{A\left(k_{h}+\epsilon k_{h}^{\prime}\right)\left(\xi+\epsilon \xi^{\prime}\right)-A\left(k_{h}\right)(\xi)}{\epsilon} & =A\left(k_{h}\right) \xi^{\prime}+\left(\lim _{\epsilon \rightarrow 0} \frac{A\left(k_{h}+\epsilon k_{h}^{\prime}\right)-A\left(k_{h}\right)}{\epsilon}\right) \xi \\
& =A\left(k_{h}\right) \xi^{\prime}+k_{h}^{\prime} C\left(k_{h}\right) \xi
\end{aligned}
$$

where

$$
\begin{aligned}
& C\left(k_{h}\right)[i, j]= \operatorname{Re}\left(-\int_{\Gamma} \int_{\Gamma \backslash\{x\}} \boldsymbol{\Phi}_{j}(y) \cdot \boldsymbol{\Phi}_{i}(x) \frac{\mathrm{e}^{\mathrm{i} k_{h}|x-y|}}{4 \pi}\left(\frac{1}{|x-y|}+\mathrm{i} k_{h}\right) \mathrm{d} s_{y} \mathrm{~d} s_{x}\right. \\
&\left.+\int_{\Gamma} \int_{\Gamma \backslash\{x\}} \operatorname{div}_{\Gamma}\left(\boldsymbol{\Phi}_{j}\right)(y) \operatorname{div}_{\Gamma}\left(\boldsymbol{\Phi}_{i}\right)(x) \frac{\mathrm{e}^{\mathrm{i} k_{h}|x-y|}}{4 \pi}\left(-\frac{1}{k_{h}^{2}|x-y|}+\frac{\mathrm{i}}{k_{h}}\right) \mathrm{d} s_{y} \mathrm{~d} s_{x}\right) . \\
& \lim _{h \rightarrow 0} \frac{\left\|\xi+h \xi^{\prime}\right\|_{l^{2}}^{2}-\|\xi\|_{l^{2}}^{2}}{h}=2\left(\xi, \xi^{\prime}\right) l_{l^{2}}
\end{aligned}
$$

The Newton iteration is the same as in Section 3.2.1.

\subsubsection{A Priori error estimates}

If we want to give a Priori error estimate for eigenvalues of (3.3.3) and (3.3.4) by Theorem 7, we need to check if $\mathbf{S}_{k}$ is a holomorphic Fredholm operator function and satisfies (3.1.1). In our work we consider electromagnetic waves in a dielectric medium, so $k$ is a real positive number. We can extend $k$ to be a complex number and the results are the same. We change the notation from $\mathbf{S}_{k}$ to $\mathbf{S}(k)$. First according the generalized Gårding inequality in Lemma 9 we can define an elliptic operator and a compact operator such that $\mathbf{S}_{k}$ can be splitted into these two operators. So $\mathbf{S}(k)$ satisfies (3.1.1). Next by using the generalized Gårding inequality, Theorem 4 in [13] and the Fredholm alternative we know that $\mathbf{S}(k)$ is a Fredholm operator with index zero for all $k \in \mathbb{C} \backslash\{0\}$. Then we give a lemma to show that $\mathbf{S}(k)$ is holomorphic. 
Lemma 18. The operator function $\mathbf{S}(k)$ is holomorphic for $k \in \mathbb{C} \backslash\{0\}$.

Proof. The proof uses Lemma 13. We define a function as

$$
\mathbf{f}_{\mathbf{v}, \mathbf{w}}(k):=\langle(\mathbf{S}(k))(\mathbf{v}), \mathbf{w}\rangle_{\tau, \Gamma} \quad \forall \mathbf{v}, \mathbf{w} \in \mathbf{W}^{-1 / 2}(\Gamma) .
$$

By using the formula for $\langle(\mathbf{S}(k))(\mathbf{v}), \mathbf{w}\rangle_{\tau, \Gamma}$ in Section 1.3.3 we have

$$
\begin{aligned}
\mathbf{f}_{\mathbf{v}, \mathbf{w}}(k)= & -k \int_{\Gamma} \int_{\Gamma} \mathbf{v}(y) \cdot \mathbf{w}(x) E_{k}(x, y) \mathrm{d} s_{y} \mathrm{~d} s_{x} \\
& +\frac{1}{k} \int_{\Gamma} \int_{\Gamma} \operatorname{div}_{\Gamma}(\mathbf{v})(y) \operatorname{div}_{\Gamma}(\mathbf{w})(x) E_{k}(x, y) \mathrm{d} s_{y} \mathrm{~d} s_{x},
\end{aligned}
$$

By using the Taylor expansion we have

$$
\mathrm{e}^{\mathrm{i} k|x-y|}=\sum_{n=0}^{\infty} \frac{(\mathrm{i} k|x-y|)^{n}}{n !} .
$$

We use $\mathrm{e}^{\mathrm{i} k|x-y|}$ in $f_{\mathbf{v}, \mathbf{w}}(k)$ and get

$$
\begin{aligned}
\mathbf{f}_{\mathbf{v}, \mathbf{w}}(k)= & \sum_{n=0}^{\infty}\left(-\int_{\Gamma} \int_{\Gamma} \mathbf{v}(y) \cdot \mathbf{w}(x) \frac{\mathbf{i}^{n}|x-y|^{n-1}}{4 \pi n !} \mathrm{d} s_{y} \mathrm{~d} s_{x}\right) k^{n+1} \\
& +\sum_{n=0}^{\infty}\left(\int_{\Gamma} \int_{\Gamma} \operatorname{div}_{\Gamma}(\mathbf{v})(y) \operatorname{div}_{\Gamma}(\mathbf{w})(x) \frac{\mathrm{i}^{n}|x-y|^{n-1}}{4 \pi n !} \mathrm{d} s_{y} \mathrm{~d} s_{x}\right) k^{n-1},
\end{aligned}
$$

So $\mathbf{f}_{\mathbf{v}, \mathbf{w}}(k)$ is a power series in $k$ and the highest order singular integral in the coefficients is a weakly singular integral for $n=0$ which is bounded. So $\mathbf{f}_{\mathbf{v}, \mathbf{w}}(k)$ is a holomorphic function for $k \in \mathbb{C} \backslash\{0\}$. The proof is done.

For the proof of a Priori error estimates of eigenvalues of (3.3.4) we can follow the same way as for the Helmholtz equation. We don't repeat these technical work and for the lowest order Raviart-Thomas boundary element space we should also get a cubic convergence.

\subsubsection{Numerical tests}

We consider the first and second eigenvalues of the Maxwell eigenvalue problem with corresponding eigenvectors on the boundary of a unit cube $(0,1)^{3}$. An analytical solution used for the test is

$$
\mathbf{u}_{k}=\left(\begin{array}{c}
\lambda_{1} \cos \left(m_{1} \pi x_{1}\right) \sin \left(m_{2} \pi x_{2}\right) \sin \left(m_{3} \pi x_{3}\right) \\
\lambda_{2} \sin \left(m_{1} \pi x_{1}\right) \cos \left(m_{2} \pi x_{2}\right) \sin \left(m_{3} \pi x_{3}\right) \\
\lambda_{3} \sin \left(m_{1} \pi x_{1}\right) \sin \left(m_{2} \pi x_{2}\right) \cos \left(m_{3} \pi x_{3}\right)
\end{array}\right)
$$

where $m_{1}, m_{2}, m_{3}$ are nonzero integers. The eigenvalue is given by

$$
\sqrt{\lambda}=k=\pi \sqrt{m_{1}^{2}+m_{2}^{2}+m_{3}^{2}} .
$$


In Tables 3.3 the first column is the level of meshes and the second column is the number of degrees of freedom, the third and fifth columns are the numerical results for the first and second eigenvalues and the fourth and sixth columns are the rate of convergence $(s)$ in $O\left(h^{2 s+1}\right)$. Fig. 3.2a is the numerical result of one eigenvector corresponding to the first eigenvalue and Fig. 3.2b is the numerical result of one eigenvector corresponding to the second eigenvalue. In the fourth and sixth columns in Table 3.3 we observe a cubic convergence approximately, since we use the lowest order Raviart-Thomas boundary element space. Our numerical results match the a Priori error estimate very well.

\begin{tabular}{|c|c|c|c|c|c|}
\hline \multicolumn{2}{|c|}{} & \multicolumn{2}{c|}{ first eigenvalue } & \multicolumn{2}{c|}{ second eigenvalue } \\
\hline level & dof & $k_{h}$ & $\mathrm{CR}_{k}$ & $k_{h}$ & $\mathrm{CR}_{k}$ \\
\hline 1 & 144 & 4.39613 & - & 5.31930 & - \\
\hline 2 & 576 & 4.43845 & 3.4004 & 5.43078 & 3.5228 \\
\hline 3 & 2304 & 4.44241 & 3.2383 & 5.44026 & 3.2217 \\
\hline$\infty$ & & 4.44288 & & 5.44140 & \\
\hline
\end{tabular}

Table 3.3 Convergence of the first eigenvalue and second eigenvalue of Maxwell eigenvalue problem with homogeneous Dirichlet boundary values

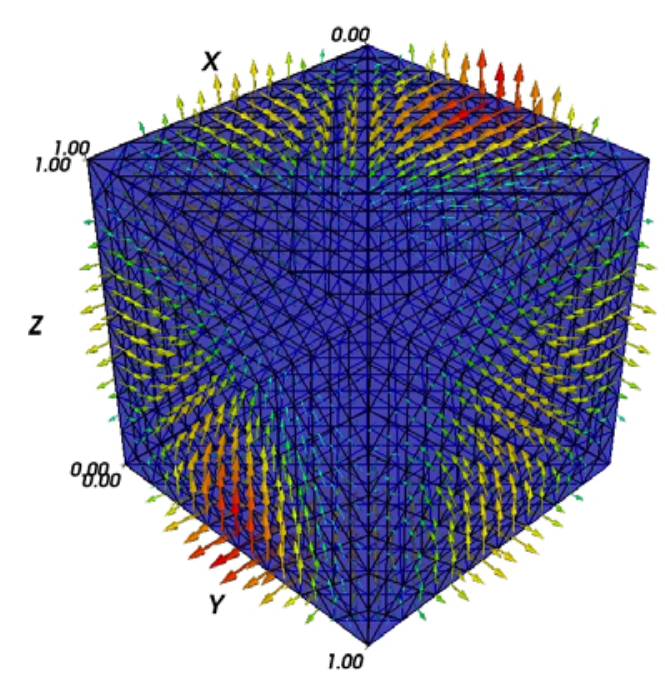

(a) $\sigma_{h}$, Neumann trace of one first eigenvector

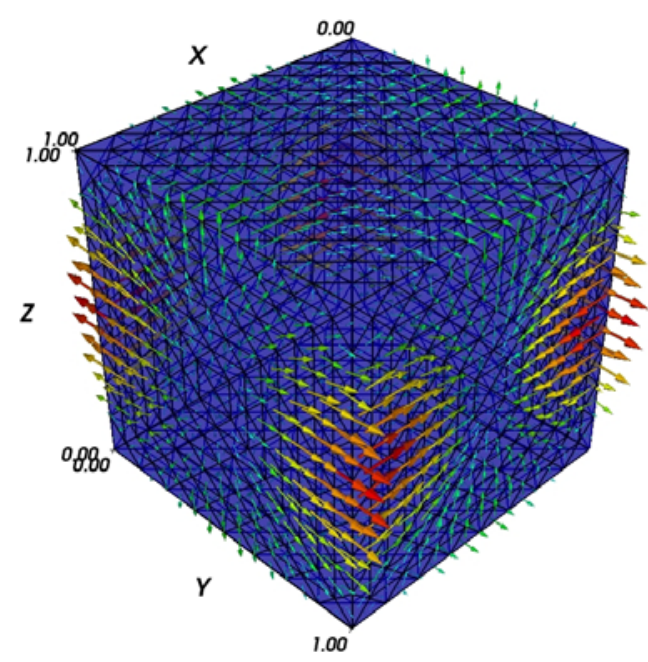

(b) $\sigma_{h}$, Neumann trace of one second eigenvector

Figure 3.2 First eigenvector and second eigenvector of Maxwell eigenvalue problem with homogeneous Dirichlet boundary condition 


\section{Chapter 4}

\section{Boundary Element Methods for Interface Eigenvalue Problems}

\subsection{The Helmholtz case}

In this section we consider the inhomogeneous problem for the Helmholtz equation in $\Omega:=\Omega^{e x} \bigcup \overline{\Omega^{i n}}$ in Section 2.1 as an eigenvalue problem and the coefficient $\alpha$ in the equation has different values in $\Omega^{e x}$ and $\Omega^{i n}$. Since we use interface conditions for the coupling in this eigenvalue problem, we call it the interface eigenvalue problem. The interface eigenvalue problem with a homogeneous Dirichlet boundary condition is to find $\lambda$ with a nontrivial solution $u$ such that

$$
\begin{aligned}
-\nabla \cdot\left(\frac{1}{\alpha} \nabla u(x)\right)-\lambda u(x) & =0 & \forall x \in \Omega^{e x} \cup \Omega^{i n}, \\
u(x) & =0 & \forall x \in \Gamma:=\partial \Omega,
\end{aligned}
$$

where $\lambda=k^{2}, k$ is a positive real number, $\alpha=\alpha^{e x}$ in $\Omega^{e x}$, and $\alpha=\alpha^{i n}$ in $\Omega^{i n} . \alpha^{e x}$ and $\alpha^{\text {in }}$ are positive constants.

We have two interface conditions for this problem

$$
\begin{aligned}
\gamma_{0}^{e x}\left(u^{e x}\right)(x) & =\gamma_{0}^{i n}\left(u^{i n}\right)(x) & & \forall x \in \Gamma^{I}, \\
\alpha^{e x} \gamma_{1}^{e x}\left(u^{e x}\right)(x) & =-\alpha^{i n} \gamma_{1}^{i n}\left(u^{i n}\right)(x) & & \forall x \in \Gamma^{I},
\end{aligned}
$$

where $u^{e x}:=\left.u\right|_{\overline{\Omega^{e x}}}, u^{i n}:=\left.u\right|_{\overline{\Omega^{i n}}}, \gamma_{0}^{e x}, \gamma_{0}^{i n}, \gamma_{1}^{e x}, \gamma_{1}^{i n}$ are Dirichlet and Neumann trace operators from $\Omega^{e x}$ and $\Omega^{i n}$, respectively, and $\Gamma^{I}:=\Gamma^{e x} \bigcap \Gamma^{i n} . \Gamma^{e x}:=\partial \Omega^{e x}$ and $\Gamma^{\text {in }}:=\partial \Omega^{i n}$.

\subsubsection{Nonlinear solution method for interface eigenvalue problem}

As in Chapter 2 we also use domain decomposition methods for this interface eigenvalue problem. We define $\sigma^{e x}:=\gamma_{1}^{e x} u^{e x}, \sigma^{i n}:=\gamma_{1}^{i n} u^{i n}, \varphi^{e x}:=\gamma_{0}^{e x} u^{e x}$, and $\varphi^{i n}:=\gamma_{0}^{i n} u^{i n}$. From (1.2.11) we have two Calderon projections for $\left(\varphi^{e x}, \sigma^{e x}\right)$ and 
$\left(\varphi^{i n}, \sigma^{i n}\right)$ respectively as

$$
\begin{aligned}
& \left(\begin{array}{c}
\varphi^{e x} \\
\sigma^{e x}
\end{array}\right)=\left(\begin{array}{cc}
\frac{1}{2} I-K_{k^{e x}}^{\Gamma^{e x}} & V_{k^{e x}}^{\Gamma^{e x}} \\
D_{k^{\Gamma^{e x}}}^{\Gamma^{\Gamma^{e x}}} & \frac{1}{2} I+K^{\prime} k^{e x}
\end{array}\right)\left(\begin{array}{c}
\varphi^{e x} \\
\sigma^{e x}
\end{array}\right), \\
& \left(\begin{array}{c}
\varphi^{i n} \\
\sigma^{i n}
\end{array}\right)=\left(\begin{array}{cc}
\frac{1}{2} I-K_{k^{i n}}^{\Gamma^{i n}} & V_{k^{i n}}^{\Gamma^{i n}} \\
D_{k^{i n}}^{\Gamma^{i n}} & \frac{1}{2} I+K^{{ }^{\Gamma^{i n}}} k^{i n}
\end{array}\right)\left(\begin{array}{c}
\varphi^{i n} \\
\sigma^{i n}
\end{array}\right),
\end{aligned}
$$

where $k^{e x}=\sqrt{\alpha^{e x}} k, k^{i n}=\sqrt{\alpha^{i n}} k$, and the definitions of boundary integral operators could be found in Section 1.2.3 on $\Gamma^{e x}$ and $\Gamma^{\text {in }}$ respectively.

We use the second equations in (4.1.3a) and (4.1.3b) in (4.1.2b) and get on $\Gamma^{I}$

$$
\alpha^{e x}\left(D_{k^{e x}}^{\Gamma^{e x}} \varphi^{e x}+\left(\frac{1}{2} I+K_{k^{\prime x x}}^{\Gamma^{e x}}\right) \sigma^{e x}\right)+\alpha^{i n}\left(D_{k^{i n}}^{\Gamma^{i n}} \varphi^{i n}+\left(\frac{1}{2} I+{K^{\prime}}_{k^{i n}}^{\Gamma^{i n}}\right) \sigma^{i n}\right)=0 .
$$

From (4.1.2a) and (4.1.1b) we have $\varphi^{e x}=\varphi^{i n}$ on $\Gamma^{I}$ and $\varphi^{e x}=0$ on $\Gamma$. We define $\varphi^{I}$ as $\varphi^{I}=\varphi^{e x}=\varphi^{\text {in }}$ on $\Gamma^{I}$ and $\varphi^{I}=0$ on $\Gamma$. We assume that the exterior unit normal on $\Gamma^{I}$ and $\Gamma$ is the same as on $\Gamma^{e x}$. We use $\varphi^{I}$ and $\varphi^{e x}=0$ on $\Gamma$ in (4.1.4) and get

$$
\begin{aligned}
\left(\alpha^{e x} D_{k^{e x}}^{\Gamma^{I}}+\alpha^{i n} D_{k^{i n}}^{\Gamma^{I}}\right)\left(\varphi^{I}\right)(x) & +\alpha^{e x}\left(\frac{1}{2} I+{K^{\prime}}_{k^{\Gamma^{e x}}}\right)\left(\sigma^{e x}\right)(x) \\
& +\alpha^{i n}\left(\frac{1}{2} I+{K^{\prime{ }^{i n}}}_{k^{i n}}\right)\left(\sigma^{i n}\right)(x)=0
\end{aligned}
$$

From the first equations in (4.1.3a) and (4.1.3b) we get

$$
\begin{gathered}
\left(\frac{1}{2} I+K_{k^{e x}}^{\Gamma^{I}}\right)\left(\varphi^{I}\right)(x)-V_{k^{e x}}^{\Gamma^{e x}}\left(\sigma^{e x}\right)(x)=0 \quad \text { a.e. } x \in \Gamma^{e x}, \\
\left(\frac{1}{2} I+K_{k^{i n}}^{\Gamma^{i n}}\right)\left(\varphi^{I}\right)(x)-V_{k^{i n}}^{\Gamma^{i n}}\left(\sigma^{i n}\right)(x)=0 \quad \text { a.e. } x \in \Gamma^{i n} .
\end{gathered}
$$

The combination of (4.1.5), (4.1.6) and (4.1.7) is the system of equations in distributional sense.

By using the left hand side of (4.1.5), (4.1.6) and (4.1.7) we define three operators

$$
\begin{aligned}
& \mathbf{F}_{\mathbf{1}}: H^{1 / 2}\left(\Gamma^{I}\right) \times H^{-1 / 2}\left(\Gamma^{e x}\right) \times H^{-1 / 2}\left(\Gamma^{i n}\right) \times \mathbb{R} \rightarrow H^{-1 / 2}\left(\Gamma^{I}\right), \\
& \mathbf{F}_{\mathbf{2}}: H^{1 / 2}\left(\Gamma^{I}\right) \times H^{-1 / 2}\left(\Gamma^{e x}\right) \times \mathbb{R} \rightarrow H^{1 / 2}\left(\Gamma^{e x}\right), \\
& \mathbf{F}_{\mathbf{3}}: H^{1 / 2}\left(\Gamma^{I}\right) \times H^{-1 / 2}\left(\Gamma^{i n}\right) \times \mathbb{R} \rightarrow H^{1 / 2}\left(\Gamma^{i n}\right),
\end{aligned}
$$

which result into the following variational problem: Find $k$ with a nontrivial solution $\left(\varphi^{I}, \sigma^{e x}, \sigma^{i n}\right) \in H^{1 / 2}\left(\Gamma^{I}\right) \times H^{-1 / 2}\left(\Gamma^{e x}\right) \times H^{-1 / 2}\left(\Gamma^{i n}\right)$ such that

$$
\begin{aligned}
& \left\langle\mathbf{F}_{1}\left(\varphi^{I}, \sigma^{e x}, \sigma^{i n}, k\right), v^{I}\right\rangle_{\Gamma^{I}}=0 \\
& \left\langle\mathbf{F}_{2}\left(\varphi^{I}, \sigma^{e x}, k\right), \chi^{e x}\right\rangle_{\Gamma^{e x}}=0 \\
& \left\langle\mathbf{F}_{3}\left(\varphi^{I}, \sigma^{i n}, k\right), \chi^{i n}\right\rangle_{\Gamma^{i n}}=0
\end{aligned}
$$


for all $\left(v^{I}, \chi^{e x}, \chi^{i n}\right) \in H^{1 / 2}\left(\Gamma^{I}\right) \times H^{-1 / 2}\left(\Gamma^{e x}\right) \times H^{-1 / 2}\left(\Gamma^{i n}\right)$.

We define a piecewise linear function space $S_{h}^{1}\left(\Gamma_{h}^{I}\right)$ and two piecewise constant function spaces $S_{h}^{0}\left(\Gamma_{h}^{e x}\right)$ and $S_{h}^{0}\left(\Gamma_{h}^{i n}\right)$ corresponding to the Sobolev spaces. The discretization of (4.1.8) is to find $k_{h}$ and a nontrivial solution $\left(\varphi_{h}^{I}, \sigma_{h}^{e x}, \sigma_{h}^{i n}\right) \in S_{h}^{1}\left(\Gamma_{h}^{I}\right) \times S_{h}^{0}\left(\Gamma_{h}^{e x}\right) \times$ $S_{h}^{0}\left(\Gamma_{h}^{i n}\right)$ such that

$$
\begin{aligned}
& \left\langle\mathbf{F}_{1}\left(\varphi_{h}^{I}, \sigma_{h}^{e x}, \sigma_{h}^{i n}, k_{h}\right), v_{h}^{I}\right\rangle_{\Gamma^{I}}=0, \\
& \left\langle\mathbf{F}_{2}\left(\varphi_{h}^{I}, \sigma_{h}^{e x}, k_{h}\right), \chi_{h}^{e x}\right\rangle_{\Gamma^{e x}}=0, \\
& \left\langle\mathbf{F}_{3}\left(\varphi_{h}^{I}, \sigma_{h}^{i n}, k_{h}\right), \chi_{h}^{i n}\right\rangle_{\Gamma^{i n}}=0,
\end{aligned}
$$

for all $\left(v_{h}^{I}, \chi_{h}^{e x}, \chi_{h}^{i n}\right) \in S_{h}^{1}\left(\Gamma_{h}^{I}\right) \times S_{h}^{0}\left(\Gamma_{h}^{e x}\right) \times S_{h}^{0}\left(\Gamma_{h}^{i n}\right)$.

Let $\left\{\Phi_{i}^{I}\right\}_{i=1}^{N^{I}},\left\{\Phi_{i}^{e x}\right\}_{i=1}^{N^{e x}}$ and $\left\{\Phi_{i}^{i n}\right\}_{i=1}^{N^{i n}}$ be the basis of $S_{h}^{1}\left(\Gamma_{h}^{I}\right), S_{h}^{0}\left(\Gamma_{h}^{e x}\right)$ and $S_{h}^{0}\left(\Gamma_{h}^{i n}\right)$. We use $\varphi_{h}^{I}=\sum_{i=1}^{N^{I}} \xi_{i}^{I} \Phi_{i}^{I}, \sigma_{h}^{e x}=\sum_{i=1}^{N^{e x}} \xi_{i}^{e x} \Phi_{i}^{e x}$ and $\sigma_{h}^{i n}=\sum_{i=1}^{N^{i n}} \xi_{i}^{i n} \Phi_{i}^{i n}$ in (4.1.9) and get a nonlinear equation system,

$$
\operatorname{Re} A\left(k_{h}\right) \xi:=\operatorname{Re}\left(\begin{array}{ccc}
A_{11}\left(k_{h}\right) & A_{12}\left(k_{h}\right) & A_{13}\left(k_{h}\right) \\
A_{21}\left(k_{h}\right) & A_{22}\left(k_{h}\right) & 0 \\
A_{31}\left(k_{h}\right) & 0 & A_{33}\left(k_{h}\right)
\end{array}\right)\left(\begin{array}{c}
\xi^{I} \\
\xi^{\text {ex }} \\
\xi^{\text {in }}
\end{array}\right)=0 .
$$

Obviously the calculation of the elements in $A\left(k_{h}\right)$ is the same as in $A$ in Section 2.1.4 and $A\left(k_{h}\right)$ is symmetric. To avoid a trivial solution we include a normalization for $\xi$. A suitable normalization is defined by

$$
\left\|\xi^{I}\right\|_{l^{2}}^{2}+\left\|\xi^{e x}\right\|_{l^{2}}^{2}+\left\|\xi^{i n}\right\|_{l^{2}}^{2}=1
$$

Then the eigenvalue problem is to solve a real nonlinear equation system

$$
\begin{aligned}
& \operatorname{Re} A\left(k_{h}\right) \xi=0 \\
& \left\|\xi^{I}\right\|_{l^{2}}^{2}+\left\|\xi^{e x}\right\|_{l^{2}}^{2}+\left\|\xi^{i n}\right\|_{l^{2}}^{2}-1=0 .
\end{aligned}
$$

We continue to use the Newton method to solve (4.1.10). The Frechet derivative is derived as the following.

$$
\lim _{h \rightarrow 0} \frac{A\left(k+h k^{\prime}\right)\left(\xi+h \xi^{\prime}\right)-A(k)(\xi)}{h}=A(k) \xi^{\prime}+k^{\prime} C(k) \xi,
$$

where

$$
\begin{gathered}
C(k)=\left(\begin{array}{ccc}
C_{11}(k) & C_{12}(k) & C_{13}(k) \\
C_{21}(k) & C_{22}(k) & 0 \\
C_{31}(k) & 0 & C_{33}(k)
\end{array}\right) \cdot \\
\operatorname{Re} C_{11}(k)[i, j]=\operatorname{Re}\left(-\alpha^{e x} \int_{\Gamma^{I}} \int_{\Gamma^{I}} \frac{\partial}{\partial \mathbf{n}_{x}}\left(\frac{\partial}{\partial \mathbf{n}_{y}}\left(\frac{\mathrm{i} \sqrt{\alpha^{e x}} \mathrm{e}^{\mathrm{i} k^{e x}|x-y|}}{4 \pi}\right)\right) \Phi_{j}^{I}(y) \Phi_{i}^{I}(x) \mathrm{d} s_{y} \mathrm{~d} s_{x}\right. \\
\left.-\alpha^{i n} \int_{\Gamma^{I}} \int_{\Gamma^{I}} \frac{\partial}{\partial \mathbf{n}_{x}}\left(\frac{\partial}{\partial \mathbf{n}_{y}}\left(\frac{\mathrm{i} \sqrt{\alpha^{i n}} \mathrm{e}^{\mathrm{i} k^{i n}|x-y|}}{4 \pi}\right)\right) \Phi_{j}^{I}(y) \Phi_{i}^{I}(x) \mathrm{d} s_{y} \mathrm{~d} s_{x}\right)
\end{gathered}
$$

The calculation of $C_{22}(k)$ and $C_{33}(k)$ is the same as in Section 3.2.1. Obviously $C_{11}(k)$, 
$C_{22}(k)$ and $C_{33}(k)$ are symmetric.

$$
\begin{aligned}
& \operatorname{Re} C_{12}(k)[i][j]=\operatorname{Re}\left(\alpha^{e x} \int_{\Gamma^{I}} \int_{\Gamma^{e x}} \frac{\partial}{\partial \mathbf{n}_{x}}\left(\frac{\mathrm{i} \sqrt{\alpha^{e x}} \mathrm{e}^{\mathrm{i} k^{e x}|x-y|}}{4 \pi}\right) \Phi_{j}^{e x}(y) \Phi_{i}^{I}(x) \mathrm{d} s_{y} \mathrm{~d} s_{x}\right) \\
& \operatorname{Re} C_{21}(k)[j][i]=\operatorname{Re}\left(\int_{\Gamma^{e x}} \int_{\Gamma^{I}} \frac{\partial}{\partial \mathbf{n}_{y}}\left(\frac{\mathrm{i} \sqrt{\alpha^{e x}} \mathrm{e}^{\mathrm{i} k^{e x}|x-y|}}{4 \pi}\right) \Phi_{i}^{I}(y) \Phi_{j}^{e x}(x) \mathrm{d} s_{y} \mathrm{~d} s_{x}\right) \\
& \operatorname{Re}_{13}(k)[i][j]=\operatorname{Re}\left(-\alpha^{i n} \int_{\Gamma^{I}} \int_{\Gamma^{i n}} \frac{\partial}{\partial \mathbf{n}_{x}^{e x}}\left(\frac{\mathrm{i} \sqrt{\alpha^{i n}} \mathrm{e}^{\mathrm{i} k^{i n}|x-y|}}{4 \pi}\right) \Phi_{j}^{i n}(y) \Phi_{i}^{I}(x) \mathrm{d} s_{y} \mathrm{~d} s_{x}\right) \\
& \operatorname{Re}_{31}(k)[j][i]=\operatorname{Re}\left(\int_{\Gamma^{i n}} \int_{\Gamma^{i n}} \frac{\partial}{\partial \mathbf{n}_{y}^{i n}}\left(\frac{\mathrm{i} \sqrt{\alpha^{i n}} \mathrm{e}^{\mathrm{i} k^{i n}|x-y|}}{4 \pi}\right) \Phi_{i}^{I}(y) \Phi_{j}^{i n}(x) \mathrm{d} s_{y} \mathrm{~d} s_{x}\right)
\end{aligned}
$$

This shows that $\operatorname{Re} C_{12}(k)[i][j]=\alpha^{e x} \operatorname{Re} C_{21}(k)[j][i]$, and $\operatorname{Re} C_{13}(k)[i][j]=\alpha^{i n} \operatorname{Re} C_{31}(k)[j][i]$ results from $\mathbf{n}^{e x}(x)=-\mathbf{n}^{i n}(x)$ for $x \in \Gamma^{I}$. So $\operatorname{Re} C(k)$ is symmetric. The integrals in $C(k)$ are more regular than in $A(k)$.

$$
\lim _{h \rightarrow 0} \frac{\left\|\xi+h \xi^{\prime}\right\|_{l^{2}}^{2}-\|\xi\|_{l^{2}}^{2}}{h}=2\left(\xi^{I}, \xi^{I^{\prime}}\right)_{l^{2}}+2\left(\xi^{e x}, \xi^{e x \prime}\right)_{l^{2}}+2\left(\xi^{i n}, \xi^{i n^{\prime}}\right)_{l^{2}}
$$

The Newton method is defined by

$$
\begin{aligned}
& \operatorname{Re} A\left(k^{n}\right)\left(\xi^{n+1}-\xi^{n}\right)+\left(k^{n+1}-k^{n}\right) \operatorname{Re} C\left(k^{n}\right) \xi^{n}+\operatorname{Re} A\left(k^{n}\right) \xi^{n}=0 \\
& 2\left(\xi^{n}, \xi^{n+1}-\xi^{n}\right) l^{2}+\|\xi\|_{l^{2}}^{2}-1=0
\end{aligned}
$$

and the iteration step is to find $\left(k^{n+1}, \xi^{n+1}\right)$ with given data $\left(k^{n}, \xi^{n}\right)$ by solving

$$
\begin{aligned}
& \operatorname{Re} A\left(k^{n}\right) \xi^{n+1}+k^{n+1} \operatorname{Re} C\left(k^{n}\right) \xi^{n}=k^{n} \operatorname{Re} C\left(k^{n}\right) \xi^{n}, \\
& 2\left(\xi^{n}, \xi^{n+1}\right)_{l^{2}}=\|\xi\|_{l^{2}}^{2}+1 .
\end{aligned}
$$

\subsubsection{Numerical tests}

We consider the first and second eigenvalues of the interface eigenvalue problem for the Laplace operator with corresponding eigenvectors on the boundary of a unit cube $(0,1)^{3}$. We assume $\alpha^{e x}=\alpha^{i n}=1$. An analytical solution used for the test is (3.2.6). The eigenvalue is $k=\pi \sqrt{m_{1}^{2}+m_{2}^{2}+m_{3}^{2}}$. In Table 4.1, the first column is the level of meshes, the second column is the number of degrees of freedom, the third and fifth columns are the absolute value of the difference between eigenvalues and their approximations, and the fourth and sixth columns are the rate of convergence $(s)$ in $O\left(h^{2 s+1}\right)$. Fig. 4.1a, 4.1b and 4.1c are the results of an eigenvector $\left(\sigma_{h}^{e x}, \varphi_{h}^{I}, \sigma_{h}^{i n}\right)$ corresponding to the first eigenvalue and Fig. 4.2a, 4.2b and 4.2c are the results of an eigenvector $\left(\sigma_{h}^{e x}, \varphi_{h}^{I}, \sigma_{h}^{i n}\right)$ corresponding to the second eigenvalue. In the fourth and sixth columns in Table 4.1 we observed that the rate of convergence is much faster than a cubic convergence. This may be explained by the better approximation of $\varphi^{I}$ with piecewise linear function spaces if the error is dominated at the interface. 


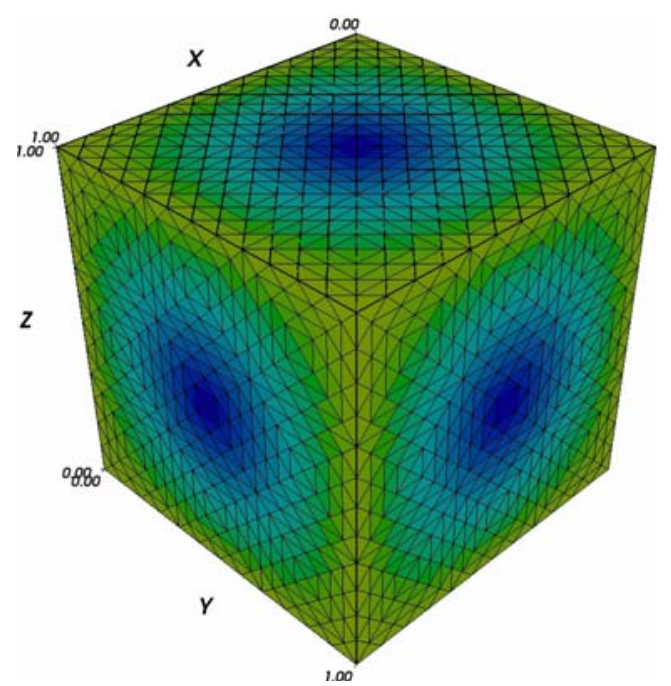

(a) $\sigma^{e x}$, Neumann trace from exterior domain

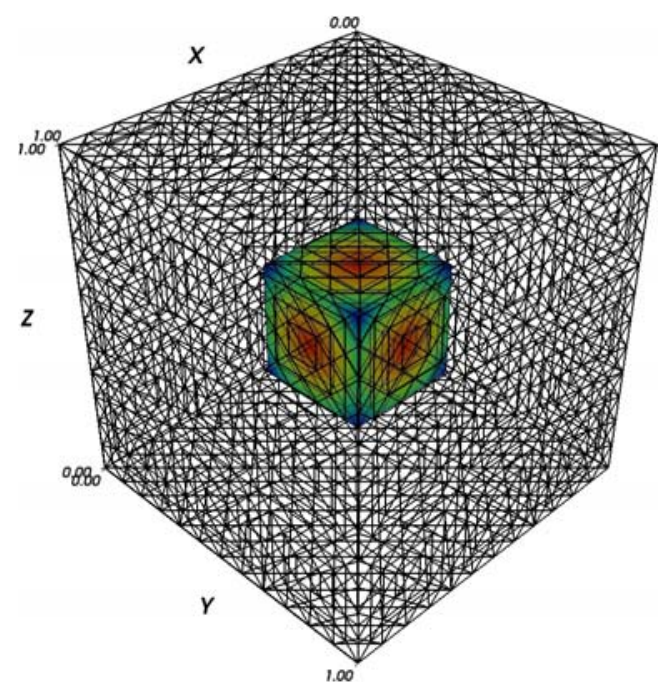

(b) $\varphi^{I}$, Dirichlet trace on interface

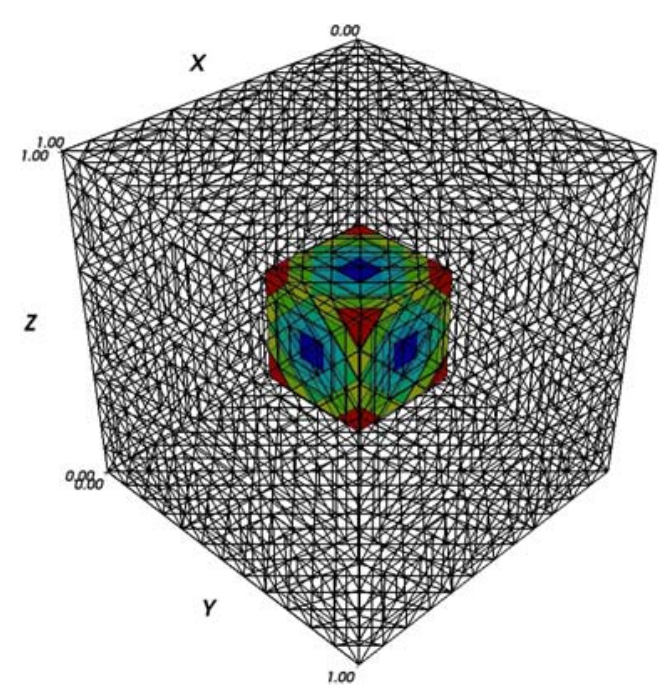

(c) $\sigma^{i n}$, Neumann trace from interior domain

Figure 4.1 First eigenvector of the interface eigenvalue problem for the Helmholtz equation with homogeneous Dirichlet boundary condition

\begin{tabular}{|c|c|c|c|c|c|}
\hline \multicolumn{2}{|c|}{} & \multicolumn{2}{c|}{ first eigenvalue } & \multicolumn{2}{c|}{ second eigenvalue } \\
\hline level & dof & $\left|k_{h}-k\right|$ & $\mathrm{CR}_{k}$ & $\left|k_{h}-k\right|$ & $\mathrm{CR}_{k}$ \\
\hline 0 & 278 & 5.43185 & - & 7.66779 & - \\
\hline 1 & 1106 & 5.44105 & 4.7890 & 7.69367 & 4.0782 \\
\hline 2 & 4418 & 5.44142 & 4.1393 & 7.69518 & 3.7372 \\
\hline$\infty$ & & 5.44140 & & 7.69530 & \\
\hline
\end{tabular}

Table 4.1 Convergence of the first eigenvalue and second eigenvalue of the interface eigenvalue problem for the Laplace equation with homogeneous Dirichlet boundary condition 


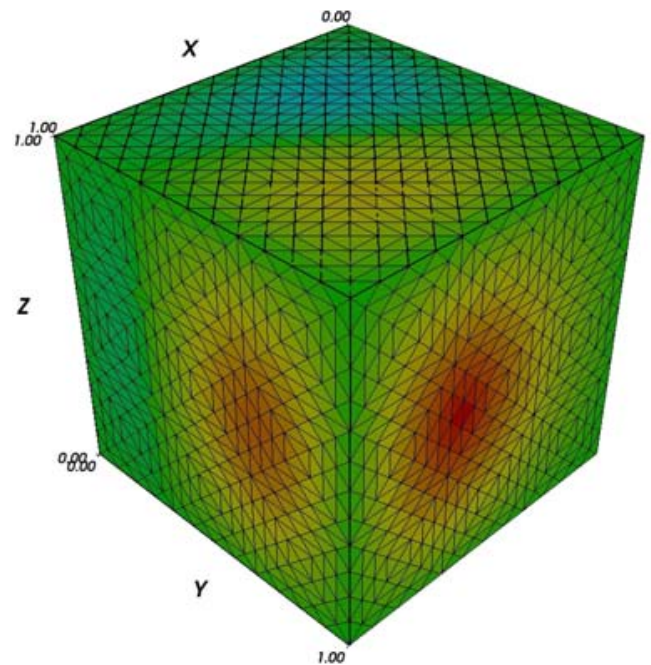

(a) $\sigma^{e x}$, Neumann trace from exterior domain

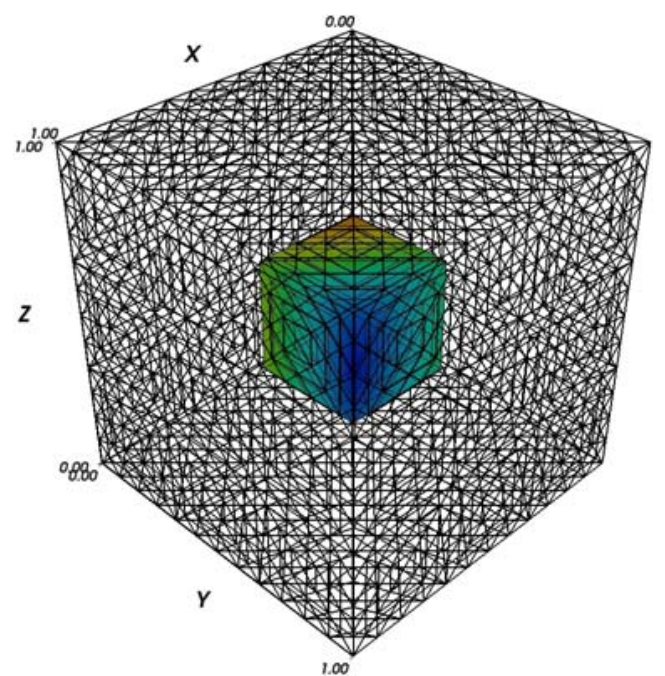

(b) $\varphi^{I}$, Dirichlet trace on interface

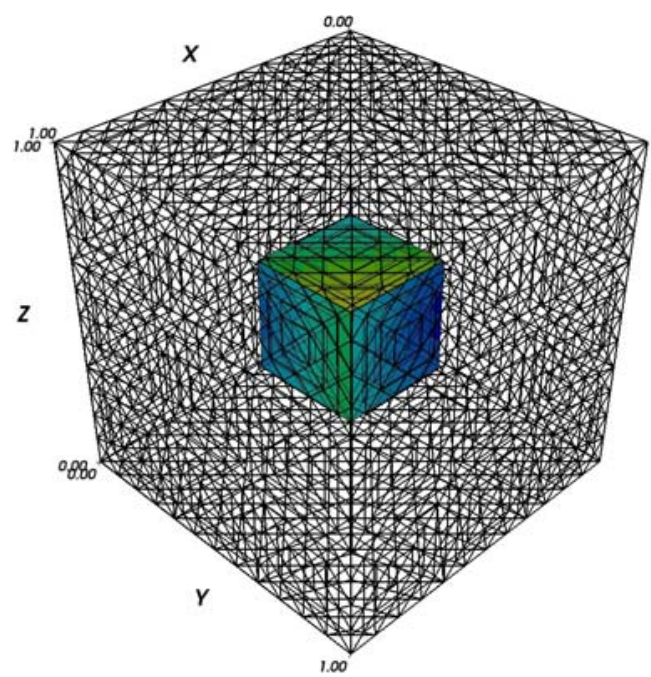

(c) $\sigma^{\text {in }}$, Neumann trace from interior domain

Figure 4.2 Second eigenvector of the interface eigenvalue problem for the Helmholtz equation with homogeneous Dirichlet boundary condition 


\subsection{The Maxwell case}

In Section 2.2 we consider electromagnetic waves in an inhomogeneous dielectric medium. In this section we consider this inhomogeneous problem for the Maxwell's equations in $\Omega:=\Omega^{e x} \cup \overline{\Omega^{i n}}$ as an eigenvalue problem and the coefficient $\alpha$ in the equation has different values in $\Omega^{e x}$ and $\Omega^{i n}$. Since we use interface conditions for this eigenvalue problem, we call it the interface eigenvalue problem. The interface eigenvalue problem with a homogeneous Dirichlet boundary condition is to find $\lambda$ with a nontrivial solution $\mathbf{u}$ such that

$$
\begin{array}{lc}
\nabla \times\left(\frac{1}{\alpha(x)} \nabla \times \mathbf{u}(x)\right)-\lambda \mathbf{u}(x)=0 & \forall x \in \Omega^{e x} \cup \Omega^{i n}, \\
\nabla \cdot \mathbf{u}(x)=0 & \forall x \in \Omega^{e x} \cup \Omega^{i n}, \\
\gamma_{\mathbf{t}}(\mathbf{u})(x)=0 & \forall x \in \Gamma:=\partial \Omega,
\end{array}
$$

where $\lambda=k^{2}, k$ is the frequence $\omega, \alpha=\alpha^{e x}=\varepsilon^{e x} \mu^{e x}$ in $\Omega^{e x}$, and $\alpha=\alpha^{i n}=\varepsilon^{i n} \mu^{i n}$ in $\Omega^{i n}$. $\varepsilon^{e x}, \varepsilon^{i n}, \mu^{e x}, \mu^{i n}$ are the permeability and permittivity in $\Omega^{e x}$ and $\Omega^{i n}$, respectively, and they are positive constants.

We have two interface conditions for this problem from (1.1.2b) and (1.1.2d),

$$
\begin{array}{ll}
\gamma_{\mathbf{t}}^{e x}\left(\mathbf{u}^{e x}\right)(x)+\gamma_{\mathbf{t}}^{i n}\left(\mathbf{u}^{i n}\right)(x)=0 & \forall x \in \Gamma^{I}, \\
\beta^{e x} \gamma_{\mathbf{N}}^{e x}\left(\mathbf{u}^{e x}\right)(x)+\beta^{i n} \gamma_{\mathbf{N}}^{i n}\left(\mathbf{u}^{i n}\right)(x)=0 & \forall x \in \Gamma^{I},
\end{array}
$$

where $\mathbf{u}^{e x}:=\left.\mathbf{u}\right|_{\Omega^{e x}}, \mathbf{u}^{i n}:=\left.\mathbf{u}\right|_{\Omega^{i n}}, \gamma_{\mathbf{t}}^{e x}, \gamma_{\mathbf{t}}^{i n}, \gamma_{\mathbf{N}}^{e x}, \gamma_{\mathbf{N}}^{i n}$ are Dirichlet and Neumann trace operators from $\Omega^{e x}$ and $\Omega^{i n}$, respectively, $\beta^{e x}=\sqrt{\frac{\varepsilon^{e x}}{\mu^{e x}}}$ and $\beta^{i n}=\sqrt{\frac{\varepsilon^{i n}}{\mu^{i n}}}$ for electric fields, and $\Gamma^{I}:=\Gamma^{e x} \bigcap \Gamma^{i n}$ with $\Gamma^{e x}:=\partial \Omega^{e x}$ and $\Gamma^{i n}:=\partial \Omega^{i n}$. The definitions of the trace operators could be found in Section 1.3.3 from $\Omega^{e x}$ and $\Omega^{i n}$, respectively.

\subsubsection{Nonlinear solution method for interface eigenvalue problem}

As in Chapter 2 we also use domain decomposition methods for this interface eigenvalue problem. We define $\boldsymbol{\sigma}^{e x}:=\gamma_{\mathbf{N}}^{e x}\left(\mathbf{u}^{e x}\right), \boldsymbol{\sigma}^{i n}:=\gamma_{\mathbf{N}}^{i n}\left(\mathbf{u}^{i n}\right), \varphi^{e x}:=\gamma_{\mathbf{t}}^{e x}\left(\mathbf{u}^{e x}\right)$ and $\varphi^{i n}:=\gamma_{\mathbf{t}}^{i n}\left(\mathbf{u}^{i n}\right)$. From (1.3.14) we define two Calderon projections for $\left(\boldsymbol{\sigma}^{e x}, \boldsymbol{\varphi}^{e x}\right)$ and $\left(\sigma^{i n}, \varphi^{i n}\right)$, respectively, as

$$
\begin{aligned}
\left(\begin{array}{l}
\boldsymbol{\varphi}^{e x} \\
\boldsymbol{\sigma}^{e x}
\end{array}\right) & =\left(\begin{array}{cc}
\frac{1}{2} I+\mathbf{C}_{k^{e x}}^{\Gamma^{e x}} & \mathbf{S}_{k^{e x}}^{\Gamma^{e x}} \\
\mathbf{S}_{k^{e x}}^{\Gamma^{e x}} & \frac{1}{2} I+\mathbf{C}_{k^{e x}}^{\Gamma^{e x}}
\end{array}\right)\left(\begin{array}{c}
\boldsymbol{\varphi}^{e x} \\
\boldsymbol{\sigma}^{e x}
\end{array}\right), \\
\left(\begin{array}{c}
\boldsymbol{\varphi}^{i n} \\
\boldsymbol{\sigma}^{i n}
\end{array}\right) & =\left(\begin{array}{cc}
\frac{1}{2} I+\mathbf{C}_{k^{i n}}^{\Gamma^{i n}} & \mathbf{S}_{k^{i n}}^{\Gamma^{i n}} \\
\mathbf{S}_{k^{i n}}^{\Gamma^{i n}} & \frac{1}{2} I+\mathbf{C}_{k^{i n}}^{\Gamma^{i n}}
\end{array}\right)\left(\begin{array}{c}
\boldsymbol{\varphi}^{i n} \\
\boldsymbol{\sigma}^{i n}
\end{array}\right),
\end{aligned}
$$

where $k^{e x}=\sqrt{\alpha^{e x}} k, k^{i n}=\sqrt{\alpha^{i n}} k$, and $\mathbf{S}_{k^{e x}}^{\Gamma^{e x}}, \mathbf{C}_{k^{e x}}^{\Gamma^{e x}}, \mathbf{S}_{k^{i n}}^{\Gamma^{i n}}, \mathbf{C}_{k^{i n}}^{\Gamma^{i n}}$ are boundary integral operators on $\Gamma^{e x}$ and $\Gamma^{i n}$ with the definitions in Section 1.3.3. 
We use the second equations of (4.2.3a) and (4.2.3b) in (4.2.2b) and get on $\Gamma^{I}$

$$
\begin{aligned}
\beta^{e x}\left(\mathbf{S}_{k^{e x}}^{\Gamma^{e x}}\left(\boldsymbol{\varphi}^{e x}\right)+\right. & \left.\left(\frac{1}{2} I+\mathbf{C}_{k^{e x}}^{\Gamma^{e x}}\right)\left(\boldsymbol{\sigma}^{e x}\right)\right) \\
& +\beta^{i n}\left(\mathbf{S}_{k^{i n}}^{\Gamma^{i n}}\left(\boldsymbol{\varphi}^{i n}\right)+\left(\frac{1}{2} I+\mathbf{C}_{k^{i n}}^{\Gamma^{i n}}\right)\left(\boldsymbol{\sigma}^{i n}\right)\right)=0 .
\end{aligned}
$$

From (4.2.2a) and (4.2.1c) we have $\varphi^{e x}=-\varphi^{i n}$ on $\Gamma^{I}$ and $\varphi^{e x}=0$ on $\Gamma$. We define $\varphi^{I}$ as $\varphi^{I}=\varphi^{e x}=-\varphi^{i n}$ on $\Gamma^{I}$ and $\varphi^{I}=0$ on $\Gamma$. We assume that the exterior unit normal on $\Gamma^{I}$ and $\Gamma$ is the same as on $\Gamma^{e x}$. We use $\varphi^{I}$ and $\varphi^{e x}=0$ on $\Gamma$ in (4.2.4) and get

$$
\begin{aligned}
\left(\beta^{e x} \mathbf{S}_{k^{e x}}^{\Gamma^{I}}+\beta^{i n} \mathbf{S}_{k^{i n}}^{\Gamma^{I}}\right)\left(\boldsymbol{\varphi}^{I}\right)(x) & +\beta^{e x}\left(\frac{1}{2} I+\mathbf{C}_{k^{e x}}^{\Gamma^{e x}}\right)\left(\boldsymbol{\sigma}^{e x}\right)(x) \\
& +\beta^{i n}\left(\frac{1}{2} I+\mathbf{C}_{k^{i n}}^{\Gamma^{i n}}\right)\left(\boldsymbol{\sigma}^{i n}\right)(x)=0
\end{aligned}
$$

We change the operator $\mathbf{S}_{k^{i n}}^{\Gamma^{i n}}$ to $-\mathbf{S}_{k^{i n}}^{\Gamma^{I}}$ in (4.2.5). From the first equations in (4.2.3a) and (4.2.3b) we get

$$
\begin{aligned}
& \left(-\frac{1}{2} I+\mathbf{C}_{k^{e x}}^{\Gamma^{I}}\right)\left(\boldsymbol{\varphi}^{I}\right)(x)+\mathbf{S}_{k^{e x}}^{\Gamma^{e x}}\left(\boldsymbol{\sigma}^{e x}\right)(x)=0 \quad \text { a.e. } x \in \Gamma^{e x}, \\
& \left(\frac{1}{2} I-\mathbf{C}_{k^{i n}}^{\Gamma^{i n}}\right)\left(\boldsymbol{\varphi}^{I}\right)(x)+\mathbf{S}_{k^{i n}}^{\Gamma^{i n}}\left(\boldsymbol{\sigma}^{i n}\right)(x)=0 \quad \text { a.e. } x \in \Gamma^{i n} .
\end{aligned}
$$

The combination of (4.2.5), (4.2.6) and (4.2.7) defines the solution of (4.2.1), an eigenvalue $k$ with it's corresponding eigenvector $\left(\boldsymbol{\varphi}^{I}, \boldsymbol{\sigma}^{e x}, \boldsymbol{\sigma}^{i n}\right)$.

By using the left hand side of (4.2.5), (4.2.6) and (4.2.7) we define three operators

$$
\begin{aligned}
& \mathbf{F}_{1}: \mathbf{W}^{-1 / 2}\left(\Gamma^{I}\right) \times \mathbf{W}^{-1 / 2}\left(\Gamma^{e x}\right) \times \mathbf{W}^{-1 / 2}\left(\Gamma^{i n}\right) \times \mathbb{R} \rightarrow \mathbf{W}^{-1 / 2}\left(\Gamma^{I}\right), \\
& \mathbf{F}_{2}: \mathbf{W}^{-1 / 2}\left(\Gamma^{I}\right) \times \mathbf{W}^{-1 / 2}\left(\Gamma^{e x}\right) \times \mathbb{R} \rightarrow \mathbf{W}^{-1 / 2}\left(\Gamma^{e x}\right), \\
& \mathbf{F}_{3}: \mathbf{W}^{-1 / 2}\left(\Gamma^{I}\right) \times \mathbf{W}^{-1 / 2}\left(\Gamma^{i n}\right) \times \mathbb{R} \rightarrow \mathbf{W}^{-1 / 2}\left(\Gamma^{i n}\right),
\end{aligned}
$$

which results into the following variational formulation:

Find $k$ with a nontrivial solution $\left(\boldsymbol{\varphi}^{I}, \boldsymbol{\sigma}^{e x}, \boldsymbol{\sigma}^{i n}\right) \in \mathbf{W}^{-1 / 2}\left(\Gamma^{I}\right) \times \mathbf{W}^{-1 / 2}\left(\Gamma^{e x}\right) \times$ $\mathbf{W}^{-1 / 2}\left(\Gamma^{\text {in }}\right)$ such that

$$
\begin{aligned}
& \left\langle\mathbf{F}_{1}\left(\boldsymbol{\varphi}^{I}, \boldsymbol{\sigma}^{e x}, \boldsymbol{\sigma}^{i n}, k\right), \boldsymbol{v}^{1}\right\rangle_{\tau, \Gamma^{I}}=0 \\
& \left\langle\mathbf{F}_{2}\left(\boldsymbol{\varphi}^{I}, \boldsymbol{\sigma}^{e x}, k\right), \boldsymbol{v}^{2}\right\rangle_{\tau, \Gamma^{e x}}=0 \\
& \left\langle\mathbf{F}_{3}\left(\boldsymbol{\varphi}^{I}, \boldsymbol{\sigma}^{i n}, k\right), \boldsymbol{v}^{3}\right\rangle_{\tau, \Gamma^{i n}}=0
\end{aligned}
$$

for all $\left(\boldsymbol{v}^{1}, \boldsymbol{v}^{2}, \boldsymbol{v}^{3}\right) \in \mathbf{W}^{-1 / 2}\left(\Gamma^{I}\right) \times \mathbf{W}^{-1 / 2}\left(\Gamma^{e x}\right) \times \mathbf{W}^{-1 / 2}\left(\Gamma^{i n}\right)$.

We use the lowest order Raviart-Thomas boundary element spaces $\mathbf{W}_{h}^{-1 / 2}\left(\Gamma_{h}^{I}\right)$, $\mathbf{W}_{h}^{-1 / 2}\left(\Gamma_{h}^{e x}\right)$ and $\mathbf{W}_{h}^{-1 / 2}\left(\Gamma_{h}^{i n}\right)$ corresponding to the Hilbert spaces. The discretization 
of (4.2.8) is to find $k_{h}$ with a nontrivial solution $\left(\boldsymbol{\varphi}_{h}^{I}, \boldsymbol{\sigma}_{h}^{e x}, \boldsymbol{\sigma}_{h}^{i n}\right) \in \mathbf{W}_{h}^{-1 / 2}\left(\Gamma_{h}^{I}\right) \times$ $\mathbf{W}_{h}^{-1 / 2}\left(\Gamma_{h}^{e x}\right) \times \mathbf{W}_{h}^{-1 / 2}\left(\Gamma_{h}^{i n}\right)$ such that

$$
\begin{aligned}
& \left\langle\mathbf{F}_{1}\left(\boldsymbol{\varphi}_{h}^{I}, \boldsymbol{\sigma}_{h}^{e x}, \boldsymbol{\sigma}_{h}^{i n}, k_{h}\right), \boldsymbol{v}_{h}^{1}\right\rangle_{\tau, \Gamma^{I}}=0, \\
& \left\langle\mathbf{F}_{2}\left(\boldsymbol{\varphi}_{h}^{I}, \boldsymbol{\sigma}_{h}^{e x}, k_{h}\right), \boldsymbol{v}_{h}^{2}\right\rangle_{\tau, \Gamma^{e x}}=0, \\
& \left\langle\mathbf{F}_{3}\left(\boldsymbol{\varphi}_{h}^{I}, \boldsymbol{\sigma}_{h}^{i n}, k_{h}\right), \boldsymbol{v}_{h}^{3}\right\rangle_{\tau, \Gamma^{i n}}=0
\end{aligned}
$$

for all $\left(\boldsymbol{v}_{h}^{1}, \boldsymbol{v}_{h}^{2}, \boldsymbol{v}_{h}^{3}\right) \in \mathbf{W}_{h}^{-1 / 2}\left(\Gamma_{h}^{I}\right) \times \mathbf{W}_{h}^{-1 / 2}\left(\Gamma_{h}^{e x}\right) \times \mathbf{W}_{h}^{-1 / 2}\left(\Gamma_{h}^{i n}\right)$.

Let $\left\{\boldsymbol{\Phi}_{i}^{I}\right\}_{i=1}^{N^{I}},\left\{\boldsymbol{\Phi}_{i}^{e x}\right\}_{i=1}^{N^{e x}}$ and $\left\{\boldsymbol{\Phi}_{i}^{i n}\right\}_{i=1}^{N^{i n}}$ be the basis of $\mathbf{W}_{h}^{-1 / 2}\left(\Gamma_{h}^{I}\right), \mathbf{W}_{h}^{-1 / 2}\left(\Gamma_{h}^{e x}\right)$ and $\mathbf{W}_{h}^{-1 / 2}\left(\Gamma_{h}^{i n}\right)$, respectively. We use $\boldsymbol{\varphi}_{h}^{I}=\sum_{i=1}^{N^{I}} \xi_{j}^{I} \boldsymbol{\Phi}_{j}^{I}, \boldsymbol{\varphi}_{h}^{e x}=\sum_{i=1}^{N^{e x}} \xi_{j}^{e x} \boldsymbol{\Phi}_{j}^{e x}$ and $\boldsymbol{\varphi}_{h}^{i n}=$ $\sum_{i=1}^{N^{i n}} \xi_{j}^{i n} \boldsymbol{\Phi}_{j}^{i n}$ in (4.2.9) and get one nonlinear system

$$
\operatorname{Re} A\left(k_{h}\right) \xi:=\operatorname{Re}\left(\begin{array}{ccc}
A_{11}\left(k_{h}\right) & A_{12}\left(k_{h}\right) & A_{13}\left(k_{h}\right) \\
A_{21}\left(k_{h}\right) & A_{22}\left(k_{h}\right) & 0 \\
A_{31}\left(k_{h}\right) & 0 & A_{33}\left(k_{h}\right)
\end{array}\right)\left(\begin{array}{c}
\xi^{I} \\
\xi^{e x} \\
\xi^{\text {in }}
\end{array}\right)=0 .
$$

The calculation of the elements in $A\left(k_{h}\right)$ is the same as in $A$ in Section 2.2.4 and $A\left(k_{h}\right)$ is symmetric. To avoid a trivial solution we include a normalization for $\xi$. A suitable normalization is defined by

$$
\left\|\xi^{I}\right\|_{l^{2}}^{2}+\left\|\xi^{e x}\right\|_{l^{2}}^{2}+\left\|\xi^{i n}\right\|_{l^{2}}^{2}=1
$$

Then the eigenvalue problem is to solve a nonlinear equation system

$$
\begin{aligned}
& \operatorname{Re} A\left(k_{h}\right) \xi=0, \\
& \left\|\xi^{I}\right\|_{l^{2}}^{2}+\left\|\xi^{e x}\right\|_{l^{2}}^{2}+\left\|\xi^{i n}\right\|_{l^{2}}^{2}-1=0 .
\end{aligned}
$$

We continue to use the Newton method to solve (4.2.10). The Frechet derivative is

$$
\lim _{h \rightarrow 0} \frac{A\left(k+h k^{\prime}\right)\left(\xi+h \xi^{\prime}\right)-A(k)(\xi)}{h}=A(k) \xi^{\prime}+k^{\prime} C(k) \xi
$$

where

$$
C(k)=\left(\begin{array}{ccc}
C_{11}(k) & C_{12}(k) & C_{13}(k) \\
C_{21}(k) & C_{22}(k) & 0 \\
C_{31}(k) & 0 & C_{33}(k)
\end{array}\right)
$$

The calculation of $C_{11}(k), C_{22}(k)$ and $C_{33}(k)$ is similar with $C(k)$ in Section 3.3.1 and they are symmetric.

$$
\begin{gathered}
C_{12}[i, j](k)=\beta^{e x} \int_{\Gamma^{I}} \int_{\Gamma^{e x} \backslash\{x\}} \nabla_{x}\left(\frac{\mathrm{i} \sqrt{\alpha^{e x}} \mathrm{e}^{\mathrm{i} k^{e x}|x-y|}}{4 \pi}\right) \cdot\left(\boldsymbol{\Phi}_{j}^{e x}(y) \times \boldsymbol{\Phi}_{i}^{I}(x)\right) \mathrm{d} s_{y} \mathrm{~d} s_{x} \\
C_{21}(k)[j, i]=\int_{\Gamma^{e x}} \int_{\Gamma^{I} \backslash\{x\}} \nabla_{x}\left(\frac{\mathrm{i} \sqrt{\alpha^{e x}} \mathrm{e}^{\mathrm{i} k^{e x}|x-y|}}{4 \pi}\right) \cdot\left(\boldsymbol{\Phi}_{i}^{I}(y) \times \boldsymbol{\Phi}_{j}^{e x}(x)\right) \mathrm{d} s_{y} \mathrm{~d} s_{x}
\end{gathered}
$$




$$
\begin{gathered}
C_{13}(k)[i, j]=-\beta^{i n} \int_{\Gamma^{I}} \int_{\Gamma^{I} \backslash\{x\}} \nabla_{x}\left(\frac{\mathrm{i} \sqrt{\alpha^{i n}} \mathrm{e}^{\mathrm{i} k^{i n}|x-y|}}{4 \pi}\right) \cdot\left(\boldsymbol{\Phi}_{j}^{I}(y) \times \boldsymbol{\Phi}_{i}^{I}(x)\right) \mathrm{d} s_{y} \mathrm{~d} s_{x} \\
C_{31}(k)[j, i]=-\int_{\Gamma^{i n}} \int_{\Gamma^{i n} \backslash\{x\}} \nabla_{x}\left(\frac{\mathrm{i} \sqrt{\alpha^{i n}} \mathrm{e}^{\mathrm{i} k^{i n}|x-y|}}{4 \pi}\right) \cdot\left(\boldsymbol{\Phi}_{i}^{I}(y) \times \boldsymbol{\Phi}_{j}^{I}(x)\right) \mathrm{d} s_{y} \mathrm{~d} s_{x}
\end{gathered}
$$

This shows $C_{12}(k)[i, j]=\beta^{e x} C_{21}(k)[j, i]$, and $C_{13}(k)[i, j]=\beta^{i n} C_{31}(k)[j, i]$ results from $\mathbf{n}^{e x}(x)=-\mathbf{n}^{i n}(x)$ for $x \in \Gamma^{I} . \mathbf{n}^{e x}$ is the exterior unit normal from $\Omega^{e x}$ and $\mathbf{n}^{\text {in }}$ is the exterior unit normal from $\Omega^{i n}$. So $C(k)$ is symmetric. The integrals in $C(k)$ are more regular than the integrals in $A(k)$.

$$
\lim _{h \rightarrow 0} \frac{\left\|\xi+h \xi^{\prime}\right\|_{l^{2}}^{2}-\|\xi\|_{l^{2}}^{2}}{h}=2\left(\xi^{I}, \xi^{I^{\prime}}\right)_{l^{2}}+2\left(\xi^{e x}, \xi^{e x \prime}\right)_{l^{2}}+2\left(\xi^{i n}, \xi^{i n^{\prime}}\right)_{l^{2}}
$$

The Newton method is defined by

$$
\begin{aligned}
& \operatorname{Re} A\left(k^{n}\right)\left(\xi^{n+1}-\xi^{n}\right)+\left(k^{n+1}-k^{n}\right) \operatorname{Re} C\left(k^{n}\right) \xi^{n}+\operatorname{Re} A\left(k^{n}\right) \xi^{n}=0, \\
& 2\left(\xi^{n}, \xi^{n+1}-\xi^{n}\right)_{l^{2}}+\left\|\xi^{n}\right\|_{l^{2}}^{2}-1=0,
\end{aligned}
$$

and the iteration step is to find $\left(k^{n+1}, \xi^{n+1}\right)$ with given data $\left(k^{n}, \xi^{n}\right)$ by solving

$$
\begin{aligned}
& \operatorname{Re} A\left(k^{n}\right) \xi^{n+1}+k^{n+1} \operatorname{Re} C\left(k^{n}\right) \xi^{n}=k^{n} \operatorname{Re} C\left(k^{n}\right) \xi^{n}, \\
& 2\left(\xi^{n}, \xi^{n+1}\right)_{l^{2}}=\left\|\xi^{n}\right\|_{l^{2}}^{2}+1 .
\end{aligned}
$$

\subsubsection{Numerical tests}

We consider the first and second eigenvalues of the interface eigenvalue problem for the Maxwell's equations with corresponding eigenvectors on the boundary of a unit cube $(0,1)^{3}$. We assume that $\varepsilon^{e x}=\varepsilon^{i n}$ and $\mu^{e x}=\mu^{i n}$. An analytical solution used for the test is (3.3.6). The eigenvalue is $k=\pi \sqrt{m_{1}^{2}+m_{2}^{2}+m_{3}^{2}}$. In Table 4.2, the first column is the level of meshes, the second column is the number of degrees of freedom, the third and fifth columns are the absolute value of the difference between eigenvalue and their approximation, and the fourth and sixth columns are the rate of convergence $(s)$ in $O\left(h^{2 s+1}\right)$. Fig. 4.3a, 4.3b and $4.3 \mathrm{c}$ are the results of an eigenvector $\left(\boldsymbol{\sigma}_{h}^{e x}, \boldsymbol{\varphi}_{h}^{I}, \boldsymbol{\sigma}_{h}^{i n}\right)$ corresponding to the first eigenvalue and Fig. 4.4a, 4.4b and 4.4c are the results of an eigenvector $\left(\boldsymbol{\sigma}_{h}^{e x}, \boldsymbol{\varphi}_{h}^{I}, \boldsymbol{\sigma}_{h}^{i n}\right)$ corresponding to the second eigenvalue. In the fourth and sixth columns in Table 4.2 we observe that the rate of convergence is cubic. 


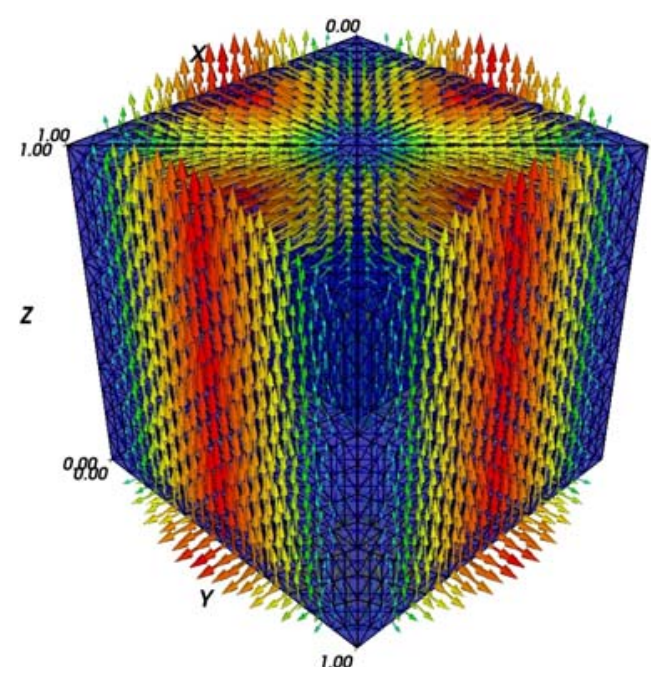

(a) $\sigma^{e x}$, Neumann trace from exterior domain

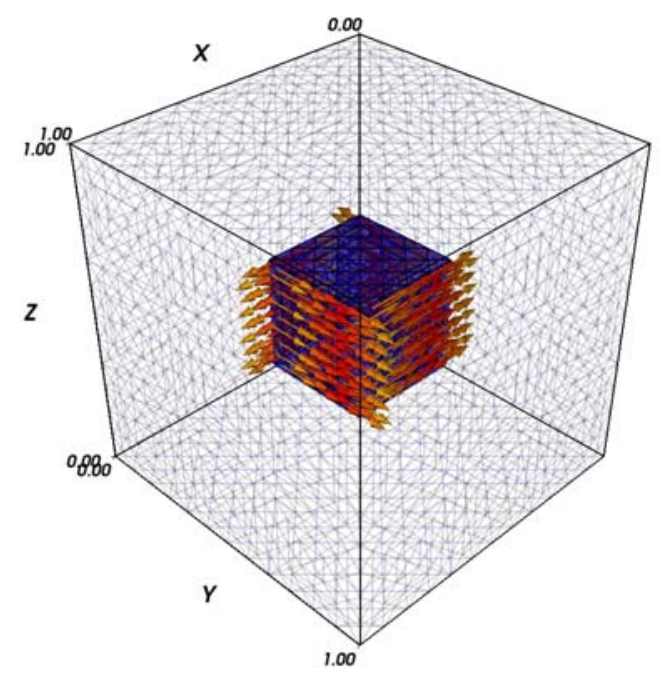

(b) $\varphi^{I}$, Dirichlet trace on interface

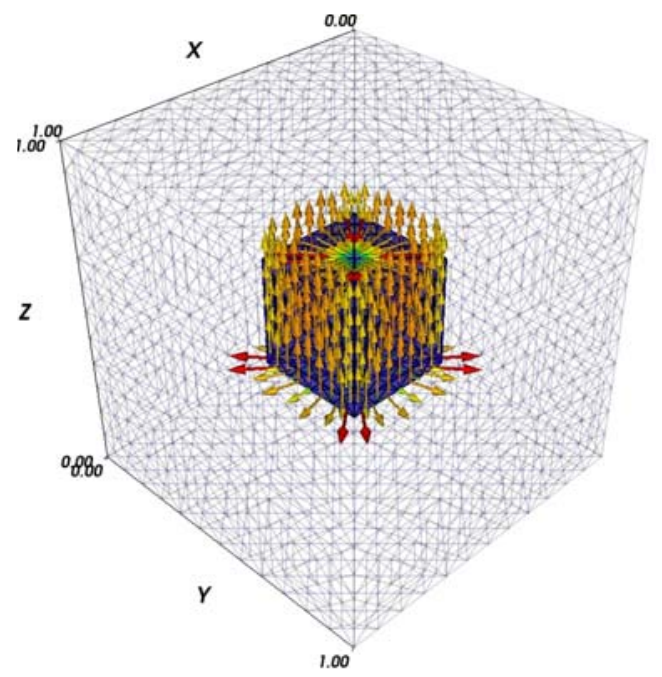

(c) $\sigma^{\text {in }}$, Neumann trace from interior domain

Figure 4.3 First eigenvector of the interface eigenvalue problem for Maxwell's equations with homogeneous Dirichlet boundary condition

\begin{tabular}{|c|c|c|c|c|c|}
\hline \multicolumn{2}{|c|}{} & \multicolumn{2}{c|}{ first eigenvalue } & \multicolumn{2}{c|}{ second eigenvalue } \\
\hline level & dof & $\left|k_{h}-k\right|$ & $\mathrm{CR}_{k}$ & $\left|k_{h}-k\right|$ & $\mathrm{CR}_{k}$ \\
\hline 0 & 432 & 4.42676 & - & 5.37587 & - \\
\hline 1 & 1728 & 4.44152 & 3.5593 & 5.43617 & 3.6474 \\
\hline 2 & 6912 & 4.44276 & 3.4450 & 5.44088 & 3.3271 \\
\hline$\infty$ & & 4.44288 & & 5.44140 & \\
\hline
\end{tabular}

Table 4.2 Convergence of the first eigenvalue and second eigenvalue of the interface eigenvalue problem for Maxwell's equations with homogeneous Dirichlet boundary condition 


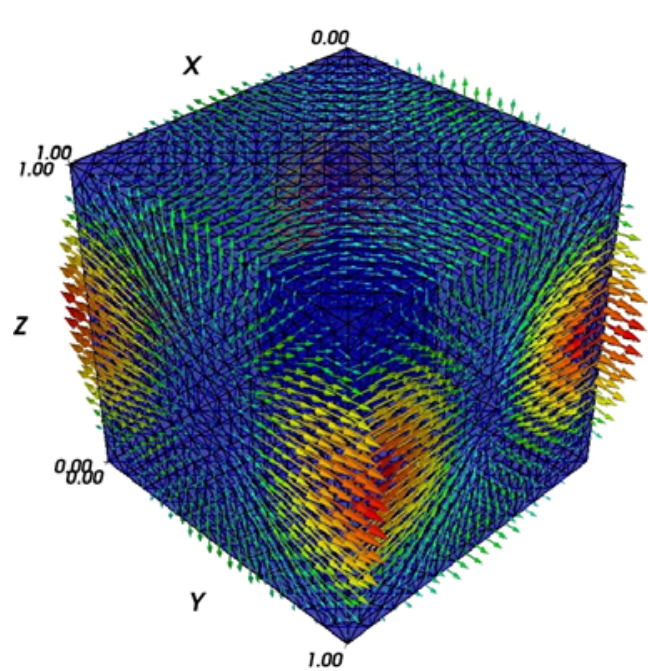

(a) $\sigma^{e x}$, Neumann trace from exterior domain

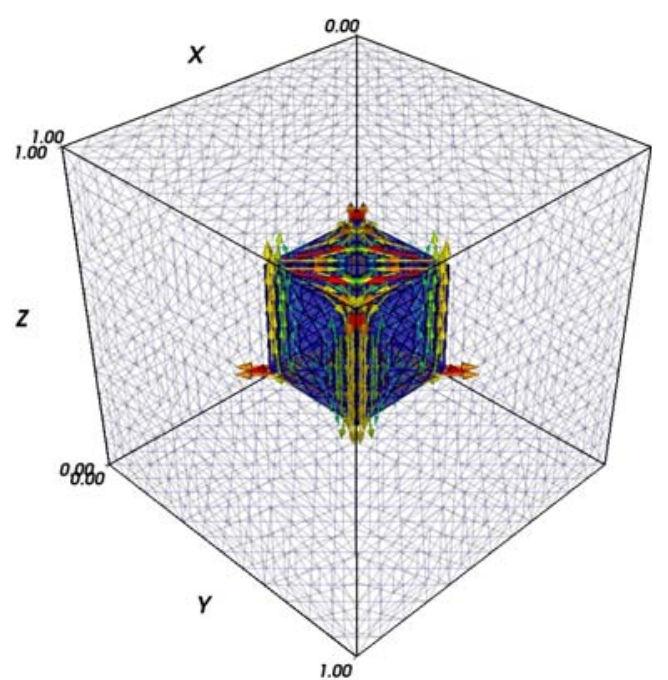

(b) $\varphi^{I}$, Dirichlet trace on interface

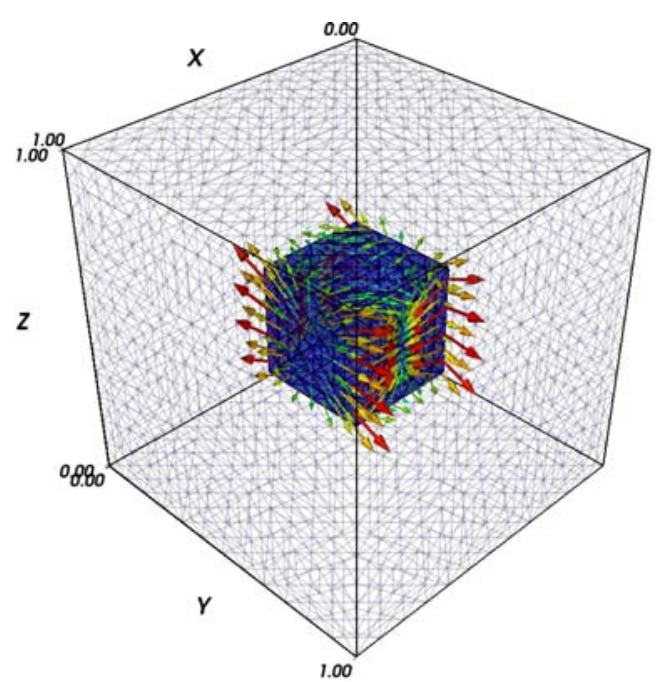

(c) $\sigma^{\text {in }}$, Neumann trace from interior domain

Figure 4.4 Second eigenvector of the interface eigenvalue problem for Maxwell's equations with homogeneous Dirichlet boundary condition 


\section{Chapter 5}

\section{Comparison of BEMs and FEMs in Band Structure Computation in 3D Photonic Crystals}

\subsection{A brief introduction to photonic crystals}

Photonic crystals are materials which are composed of periodic dielectric or metallodielectric nanostructures. They exist in nature and can also be technically produced. Photonic crystals have been studied for more than one hundred years, but the term "Photonic crystals" was first used in 1987 in [77] and [40]. In this section we introduce some background about photonic crystals to define the final problem in this thesis. The study of photonic crystals could follow [38]. First we give a definition of photonic crystals from [45].

Definition 11. Photonic crystals are periodic optical nanostructures that are designed to affect the motion of photons in a similar way that periodicity of a semiconductor crystal affects the motion of electrons.

Since photonic crystals affect the motion of photons, the Maxwell's equations are used to describe this phenomena. Since semiconductor crystals affect the motion of electrons, the Schrödinger equation is used to describe this phenomena. The motion of electrons in semiconductor crystals is studied in solid physics [36], and photonic crystals and semiconductor crystals affect the motion of photons and electrons, respectively, in a similar way. So the knowledge of solid physics is very useful for the understanding of photonic crystals. For the beginning, we have the following questions from Definition 11.

1. What is the definition of crystals?

2. How does the periodic structure of photonic crystals affect the motion of photons?

3. How to use PDEs to describe a problem in a periodic structure?

First, from solid physics we have a very simple definition of crystals for the first question:

$$
\text { Crystals }=\text { Lattice }+ \text { Basis . }
$$




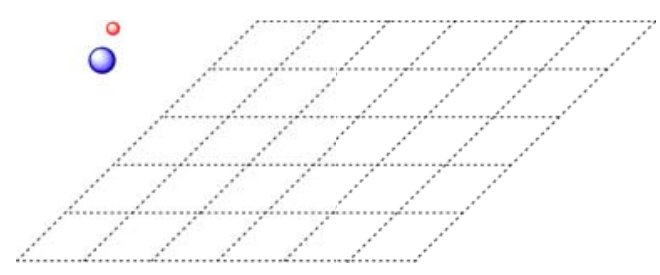

(a) lattice and basis

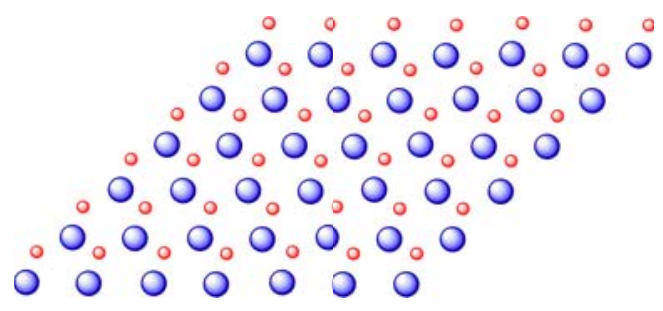

(b) crystals

Figure 5.1 A simple definition of crystals

Figure 5.1 is an example of the definition of crystals. Figure 5.1a gives a basis and a lattice and Figure 5.1b is the structure of crystals. From Figure 5.1 it is obviously that crystals have periodic structures. We can use the same way to define periodic structures for photonic crystals. The difference between crystals and photonic crystals is that the periodic structures of crystals are built by atoms and the periodic structures of photonic crystals are built by dielectric materials. The following figures are 1D, 2D and 3D periodic structures for photonic crystals from [38].

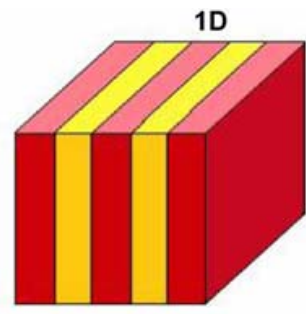

(a)

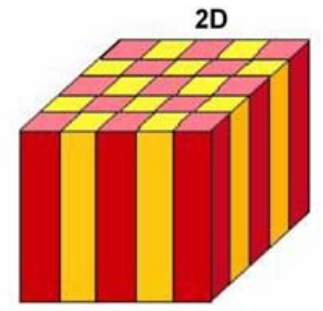

(b)

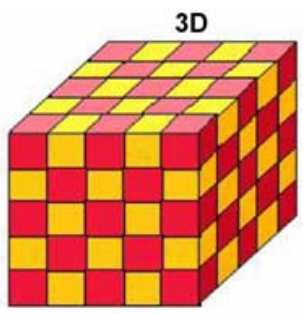

(c)

Figure 5.2 1D, 2D and 3D periodic structures of photonic crystals

The different colors in Figures 5.2 (a), (b) and (c) mean the jump of the properties of dielectric materials. For dielectric materials, the properties are the permeability $\mu$ and permittivity $\varepsilon$. By the periodicity, $\mu$ and $\varepsilon$ are periodic functions. We should pay attention that Figures 5.2 (a), (b) and (c) just show an abstract meaning of the periodicity and in fact the dielectric material in one unit cell of photonic crystals is inhomogeneous.

Next, we go to the second question. It is found that electromagnetic waves can not propagate through photonic crystals for some frequencies by Bragg diffraction because of the periodicity. This means that we can use photonic crystals to control the flow of light and this is the reason for us to produce photonic crystals. If we want to design and produce photonic crystals, we need to know for which frequencies electromagnetic waves can not propagate. So we need to calculate the frequencies and this is an eigenvalue problem obviously.

Then, we go to the third question. It looks very complex to solve the Maxwell's equations in the whole domain which has different permeabilities and permittivities in so many sub-domains. So we change the problem from the whole domain to one unit cell by the famous Bloch theorem. We give a brief introduction of the Bloch theorem for the Schrödinger equation in a periodic structure from [36] and we can use the same results to 
the Maxwell's equations. We have the Schrödinger equation in a periodic structure as

$$
H \psi(x):=\left(-\frac{\hbar^{2}}{2 m} \Delta+V(x)\right) \psi(x)=E \psi(x),
$$

where $H$ is called the Hamiltonian operator, $\hbar$ is the reduced Planck constant, $m$ is the mass of the particle, $V$ is the potential energy, $E$ is the energy and $\psi$ is the wave function for the particle. $V$ is a periodic potential from the periodic structure such that

$$
V\left(x+\mathbf{R}^{n}\right)=V(x),
$$

where $\mathbf{R}^{n}=n_{1} \mathbf{a}_{1}+n_{2} \mathbf{a}_{2}+n_{3} \mathbf{a}_{3}$ with $n_{1}, n_{2}, n_{3} \in \mathbb{N}^{0} .\left\{\mathbf{a}_{1}, \mathbf{a}_{2}, \mathbf{a}_{3}\right\}$ is a basis of one unit cell.

We define an operator $T\left(\mathbf{R}^{n}\right)$ as

$$
T\left(\mathbf{R}^{n}\right) f(x)=f\left(x+\mathbf{R}^{n}\right),
$$

where $f$ is any function. We use $T\left(\mathbf{R}^{n}\right)$ to the Hamiltonian operator and get

$$
\begin{aligned}
T\left(\mathbf{R}^{n}\right)\left(-\frac{\hbar^{2}}{2 m} \Delta+V(x)\right) \psi(x) & =\left(-\frac{\hbar^{2}}{2 m} \Delta+V\left(x+\mathbf{R}^{n}\right)\right) \psi\left(x+\mathbf{R}^{n}\right) \\
& =\left(-\frac{\hbar^{2}}{2 m} \Delta+V(x)\right) \psi\left(x+\mathbf{R}^{n}\right) \\
& =\left(-\frac{\hbar^{2}}{2 m} \Delta+V(x)\right) T\left(\mathbf{R}^{n}\right) \psi(x) .
\end{aligned}
$$

The second equality is from (5.1.2). So the eigenvalue problem for the Hamiltonian operator is equivalent to the eigenvalue problem for $T\left(\mathbf{R}^{n}\right)$. The eigenvalue problem for $T\left(\mathbf{R}^{n}\right)$ is

$$
T\left(\mathbf{R}^{n}\right) \psi(x)=\lambda^{n} \psi(x) .
$$

By the periodic structure, we have

$$
\begin{aligned}
\left|\psi\left(x+\mathbf{R}^{n}\right)\right|=\left|T\left(\mathbf{R}^{n}\right) \psi(x)\right|=\left|\lambda^{n} \psi(x)\right| & =|\psi(x)|, \\
\left|\lambda^{n}\right| & =1 .
\end{aligned}
$$

So we can write $\lambda^{n}$ as

$$
\lambda^{n}=\mathrm{e}^{\mathrm{i} \boldsymbol{\alpha} \cdot \mathbf{R}^{n}},
$$

where $\boldsymbol{\alpha}$ is any vector. By using (5.1.4) in (5.1.3), we get the Bloch theorem for the Schrödinger equation

$$
\psi\left(x+\mathbf{R}^{n}\right)=\mathrm{e}^{\mathrm{i} \boldsymbol{\alpha} \cdot \mathbf{R}^{n}} \psi(x) .
$$

We continue to consider the Maxwell's equations. By the periodic structure, we have

$$
\begin{aligned}
\varepsilon\left(x+\mathbf{R}^{n}\right) & =\varepsilon(x), \\
\mu\left(x+\mathbf{R}^{n}\right) & =\mu(x) .
\end{aligned}
$$


If we consider the time-harmonic Maxwell's equations for magnetic fields, we have

$$
\begin{aligned}
& \frac{1}{\mu} \nabla \times\left(\frac{1}{\varepsilon} \nabla \times \mathbf{u}\right)=\omega^{2} \mathbf{u}, \\
& \nabla \cdot \mathbf{u}=0
\end{aligned}
$$

From (5.1.6), it is obviously that the operator on the left hand side of (5.1.7) is also a periodic operator and we can repeat all the same things for (5.1.7) as for the Schrödinger equation. We have the Bloch theorem for the Maxwell's equations as

$$
\mathbf{u}\left(x+\mathbf{R}^{n}\right)=\mathrm{e}^{\mathrm{i} \boldsymbol{\alpha} \cdot \mathbf{R}^{n}} \mathbf{u}(x) .
$$

As a summary we define our final problem. Let $\Omega:=\Omega^{e x} \cup \overline{\Omega^{i n}}$ be a primitive cell and be a unit cube. $\Gamma:=\partial \Omega$. For this primitive cell, $\mathbf{a}_{1}=(1,0,0), \mathbf{a}_{2}=(0,1,0)$ and $\mathbf{a}_{3}=(0,0,1)$ and we change the notation from a to e. Let $\Gamma_{1}=[0,1] \times[0,1] \times\{0\}$, $\Gamma_{2}=[0,1] \times\{0\} \times[0,1]$ and $\Gamma_{3}=\{0\} \times[0,1] \times[0,1]$. By using the Bloch theorem, we have the Maxwell's equations with quasi-periodic boundary conditions as the following.

$$
\begin{array}{rlrl}
\frac{1}{\mu} \nabla \times\left(\frac{1}{\varepsilon} \nabla \times \mathbf{u}\right) & =\omega^{2} \mathbf{u} & \forall x \in \Omega^{e x} \cup \Omega^{i n} \\
\nabla \cdot \mathbf{u} & =0 & \forall x \in \Omega^{e x} \cup \Omega^{i n} \\
\gamma_{\mathbf{t}}^{e x}\left(\mathbf{u}^{e x}\right)\left(x+\mathbf{e}_{i}\right) & =-\mathrm{e}^{\mathrm{i} \boldsymbol{\alpha} \cdot \mathbf{e}_{i}} \gamma_{\mathbf{t}}^{e x}\left(\mathbf{u}^{e x}\right)(x) & \text { a.e. } x & \in \Gamma_{i}, i=1,2,3 \\
\gamma_{\mathbf{N}}^{e x}\left(\mathbf{u}^{e x}\right)\left(x+\mathbf{e}_{i}\right) & =-\mathrm{e}^{\mathrm{i} \boldsymbol{\alpha} \cdot \mathbf{e}_{i}} \gamma_{\mathbf{N}}^{e x}\left(\mathbf{u}^{e x}\right)(x) & \text { a.e. } x \in \Gamma_{i}, i=1,2,3
\end{array}
$$

As we have mentioned in the first question, the dielectric material in one unit cell of photonic crystals is inhomogeneous. So we need interface conditions as the following,

$$
\begin{array}{ll}
\gamma_{\mathbf{t}}^{e x}\left(\mathbf{u}^{e x}\right)(x)+\gamma_{\mathbf{t}}^{i n}\left(\mathbf{u}^{i n}\right)(x)=0 & \text { a.e. } x \in \Gamma^{I}, \\
\beta^{e x} \gamma_{\mathbf{N}}^{e x}\left(\mathbf{u}^{e x}\right)(x)+\beta^{i n} \gamma_{\mathbf{N}}^{i n}\left(\mathbf{u}^{i n}\right)(x)=0 & \text { a.e. } x \in \Gamma^{I},
\end{array}
$$

where $\mathbf{u}^{e x}:=\left.\mathbf{u}\right|_{\overline{\Omega^{e x}}}, \mathbf{u}^{i n}:=\left.\mathbf{u}\right|_{\overline{\Omega^{i n}}}, \gamma_{\mathbf{t}}^{e x}, \gamma_{\mathbf{t}}^{i n}, \gamma_{\mathbf{N}}^{e x}, \gamma_{\mathbf{N}}^{i n}$ are Dirichlet and Neumann trace operators from $\Omega^{e x}$ and $\Omega^{i n}$ respectively, $\beta^{e x}=\sqrt{\frac{\mu^{e x}}{\varepsilon^{e x}}}, \beta^{i n}=\sqrt{\frac{\mu^{i n}}{\varepsilon^{i n}}}$ for magnetic fields, and $\Gamma^{I}:=\Gamma^{e x} \bigcap \Gamma^{i n}$ with $\Gamma^{e x}:=\partial \Omega^{e x}$ and $\Gamma^{i n}:=\partial \Omega^{i n}$. As we have mentioned in the second question, we solve (5.1.9) as an eigenvalue problem. So our final problem is an interface eigenvalue problem for the Maxwell's equations with quasi-periodic boundary conditions.

\subsection{A homogeneous problem with periodic boundary conditions}

It is too complex to solve (5.1.9) with (5.1.10) directly. So in this section we simplify the problem to a homogeneous problem with periodic boundary conditions and in the next section we consider an inhomogeneous problem with periodic boundary conditions. For 
a homogeneous problem, we assume that $\varepsilon^{e x}=\varepsilon^{i n}=\mu^{e x}=\mu^{i n}=1$ and $\boldsymbol{\alpha}=(0,0,0)$ in (5.1.9). Quasi-periodic boundary conditions in (5.1.9c) and (5.1.9d) are simplified to periodic boundary conditions and we don't need interface conditions (5.1.10) for this problem. Then the Maxwell's equations (5.1.9) with interface conditions (5.1.10) are changed to

$$
\begin{array}{lr}
\nabla \times \nabla \times \mathbf{u}(x)=\lambda \mathbf{u}(x) & \forall x \in \Omega, \\
\nabla \cdot \mathbf{u}(x)=0 & \forall x \in \Omega, \\
\gamma_{\mathbf{t}}(\mathbf{u})\left(x+\mathbf{e}_{i}\right)=-\gamma_{\mathbf{t}}(\mathbf{u})(x) & \forall x \in \Gamma_{i}, i=1,2,3, \\
\gamma_{\mathbf{N}}(\mathbf{u})\left(x+\mathbf{e}_{i}\right)=-\gamma_{\mathbf{N}}(\mathbf{u})(x) & \forall x \in \Gamma_{i}, i=1,2,3 .
\end{array}
$$

where $\lambda=k^{2}=\mu \varepsilon \omega^{2}$.

\subsubsection{Nonlinear solution method}

We define $\varphi:=\gamma_{\mathbf{t}}(\mathbf{u})$ and $\boldsymbol{\sigma}:=\gamma_{\mathbf{N}}(\mathbf{u})$. From (1.3.14) we have a Calderón projection for $(\varphi, \sigma)$

$$
\left(\begin{array}{cc}
\left(\frac{1}{2} I+\mathbf{C}_{k}\right) & \mathbf{S}_{k} \\
\mathbf{S}_{k} & \left(\frac{1}{2} I+\mathbf{C}_{k}\right)
\end{array}\right)\left(\begin{array}{c}
\boldsymbol{\varphi} \\
\boldsymbol{\sigma}
\end{array}\right)=\left(\begin{array}{c}
\boldsymbol{\varphi} \\
\boldsymbol{\sigma}
\end{array}\right) .
$$

By using (5.2.2), the eigenvalue problem (5.2.1) is to find $k \in \mathbb{R}^{+}$with a nontrivial solution $(\boldsymbol{\varphi}, \boldsymbol{\sigma})$ such that

$$
\begin{array}{ll}
\left(-\frac{1}{2} I+\mathbf{C}_{k}\right)(\boldsymbol{\varphi})(x)+\mathbf{S}_{k}(\boldsymbol{\sigma})(x)=0 & \text { a.e. } x \in \Gamma, \\
\mathbf{S}_{k}(\boldsymbol{\varphi})(x)+\left(-\frac{1}{2} I+\mathbf{C}_{k}\right)(\boldsymbol{\sigma})(x)=0 & \text { a.e. } x \in \Gamma,
\end{array}
$$

where $\varphi$ and $\sigma$ satisfy the periodic boundary conditions in (5.2.1c) and (5.2.1d). The variational formulation for (5.2.3) is to find $k \in \mathbb{R}^{+}$with a nontrivial solution $(\boldsymbol{\varphi}, \boldsymbol{\sigma}) \in$ $\mathbf{W}^{-1 / 2}(\Gamma) \times \mathbf{W}^{-1 / 2}(\Gamma)$ such that

$$
\begin{aligned}
& \left\langle\left(-\frac{1}{2} I+\mathbf{C}_{k}\right)(\boldsymbol{\varphi})+\mathbf{S}_{k}(\boldsymbol{\sigma}), \boldsymbol{v}^{1}\right\rangle_{\tau, \Gamma}=0 \\
& \left\langle\mathbf{S}_{k}(\boldsymbol{\varphi})+\left(-\frac{1}{2} I+\mathbf{C}_{k}\right)(\boldsymbol{\sigma}), \boldsymbol{v}^{2}\right\rangle_{\tau, \Gamma}=0
\end{aligned}
$$

for all $\left(\boldsymbol{v}^{1}, \boldsymbol{v}^{2}\right) \in \mathbf{W}^{-1 / 2}(\Gamma) \times \mathbf{W}^{-1 / 2}(\Gamma) . \boldsymbol{\varphi}$ and $\boldsymbol{\sigma}$ satisfy periodic boundary conditions. The Galerkin formulation for (5.2.4) is to find $k_{h} \in \mathbb{R}^{+}$with a nontrivial solution $\left(\boldsymbol{\varphi}_{h}, \boldsymbol{\sigma}_{h}\right) \in \mathbf{W}_{h}^{-1 / 2}\left(\Gamma_{h}\right) \times \mathbf{W}_{h}^{-1 / 2}\left(\Gamma_{h}\right)$ such that

$$
\begin{aligned}
& \left\langle\left(-\frac{1}{2} I+\mathbf{C}_{k_{h}}\right)\left(\boldsymbol{\varphi}_{h}\right)+\mathbf{S}_{k_{h}}\left(\boldsymbol{\sigma}_{h}\right), \boldsymbol{v}_{h}^{1}\right\rangle_{\tau, \Gamma}=0, \\
& \left\langle\mathbf{S}_{k_{h}}\left(\boldsymbol{\varphi}_{h}\right)+\left(-\frac{1}{2} I+\mathbf{C}_{k_{h}}\right)\left(\boldsymbol{\sigma}_{h}\right), \boldsymbol{v}_{h}^{2}\right\rangle_{\tau, \Gamma}=0,
\end{aligned}
$$


for all $\left(\boldsymbol{v}_{h}^{1}, \boldsymbol{v}_{h}^{2}\right) \in \mathbf{W}_{h}^{-1 / 2}\left(\Gamma_{h}\right) \times \mathbf{W}_{h}^{-1 / 2}\left(\Gamma_{h}\right) . \quad \boldsymbol{\varphi}_{h}$ and $\boldsymbol{\sigma}_{h}$ satisfy periodic boundary conditions.

Let $\left\{\boldsymbol{\Phi}_{i}\right\}_{i=1}^{N}$ be a basis of $\mathbf{W}_{h}^{-1 / 2}\left(\Gamma_{h}\right)$. We use $\boldsymbol{\varphi}_{h}=\sum_{i=1}^{N} \xi_{i}^{\mathbf{t}} \boldsymbol{\Phi}_{i}$ and $\boldsymbol{\sigma}_{h}=\sum_{i=1}^{N} \xi_{i}^{\mathbf{N}} \boldsymbol{\Phi}_{i}$ in the Galerkin formulation (5.2.5) and get a nonlinear equation system

$$
\operatorname{Re} A \xi:=\left(\begin{array}{ll}
\operatorname{Re} A_{11}\left(k_{h}\right) & \operatorname{Re} A_{12}\left(k_{h}\right) \\
\operatorname{Re} A_{21}\left(k_{h}\right) & \operatorname{Re} A_{22}\left(k_{h}\right)
\end{array}\right)\left(\begin{array}{c}
\xi^{\mathbf{t}} \\
\xi^{\mathbf{N}}
\end{array}\right)=0
$$

where $\xi^{\mathbf{t}}$ and $\xi^{\mathbf{N}}$ satisfy the periodic boundary conditions. The calculation of the elements in $A$ is similar with Section 3.3.1 and Section 4.2.1. We define $\widehat{\Gamma}_{1}:=(0,1) \times(0,1) \times\{0\}$, $\widehat{\Gamma}_{2}:=(0,1) \times\{0\} \times(0,1)$ and $\widehat{\Gamma}_{3}:=\{0\} \times(0,1) \times(0,1)$. We define $\Gamma_{12}:=\Gamma_{1} \bigcap \Gamma_{2}$, $\Gamma_{13}:=\Gamma_{1} \bigcap \Gamma_{3}$ and $\Gamma_{23}:=\Gamma_{2} \bigcap \Gamma_{3}$. We define $\Gamma^{\text {per }}:=\widehat{\Gamma}_{1} \bigcup \widehat{\Gamma}_{2} \bigcup \widehat{\Gamma}_{3} \bigcup \Gamma_{12} \bigcup \Gamma_{13} \bigcup \Gamma_{23}$. Let $\left\{\boldsymbol{\Phi}_{i_{k}}\right\}_{k=1}^{M}$ be a basis of $\mathbf{W}_{h}^{-1 / 2}\left(\Gamma_{h}^{p e r}\right)$. By using the periodic boundary conditions, we could define a mapping $B^{N \times M}$ such that

$$
\xi^{\mathbf{t}}=B \xi_{p e r}^{\mathbf{t}} \quad \text { and } \quad \xi^{\mathbf{N}}=B \xi_{p e r}^{\mathbf{N}},
$$

where $\xi_{p e r}^{\mathbf{t}}$ and $\xi_{p e r}^{\mathbf{N}}$ are the coefficients of the testing functions defined on $\Gamma^{p e r}$. We use the mapping (5.2.7) in (5.2.6) and get

$$
A^{\text {per }}\left(k_{h}\right) \xi^{\text {per }}:=\left(\begin{array}{cc}
B^{T} \operatorname{Re} A_{11}\left(k_{h}\right) B & B^{T} \operatorname{Re} A_{12}\left(k_{h}\right) B \\
B^{T} \operatorname{Re} A_{21}\left(k_{h}\right) B & B^{T} \operatorname{Re} A_{22}\left(k_{h}\right) B
\end{array}\right)\left(\begin{array}{c}
\xi_{\text {per }}^{\mathbf{t}} \\
\xi_{\text {per }}^{\mathrm{N}}
\end{array}\right)=0
$$

To avoid a trivial solution, we include a suitable normalization for $\xi^{\text {per }}$ by $\left\|\xi^{p e r}\right\|_{l^{2}}=1$. The nonlinear equation system is

$$
\begin{aligned}
& A^{\text {per }}(k) \xi^{p e r}=0 \\
& \left\|\xi^{\text {per }}\right\|_{l^{2}}^{2}-1=0
\end{aligned}
$$

We continue to use the Newton method for (5.2.8), and the Frechet derivative and the iteration steps are the same as in Section 3.3.1 and Section 4.2.1.

\subsubsection{Numerical tests}

We consider the first and second eigenvalues of the periodic eigenvalue problem with corresponding eigenvectors on the boundary of a unit cube $(0,1)^{3}$. An analytical solution used for the test is

$$
\mathbf{u}_{k}=\left(\begin{array}{c}
\lambda_{1} \cos \left(2 m_{1} \pi x_{1}\right) \sin \left(2 m_{2} \pi x_{2}\right) \sin \left(2 m_{3} \pi x_{3}\right) \\
\lambda_{2} \sin \left(2 m_{1} \pi x_{1}\right) \cos \left(2 m_{2} \pi x_{2}\right) \sin \left(2 m_{3} \pi x_{3}\right) \\
\lambda_{3} \sin \left(2 m_{1} \pi x_{1}\right) \sin \left(2 m_{2} \pi x_{2}\right) \cos \left(2 m_{3} \pi x_{3}\right)
\end{array}\right) .
$$

The eigenvalue is given by $k=2 \pi \sqrt{m_{1}^{2}+m_{2}^{2}+m_{3}^{2}}$ with $m_{1}, m_{2}, m_{3} \in \mathbb{N}^{0}$. In Table 5.1 the first column is the level of meshes and the second column is the number of degrees of freedom, the third and fifth columns are the numerical results for the first and second eigenvalues and the fourth and sixth columns are the rate of convergence. Fig. 5.3a and 
$5.3 \mathrm{~b}$ are the numerical results of one eigenvector corresponding to the first eigenvalue and Fig. $5.3 \mathrm{c}$ and $5.3 \mathrm{~d}$ are the numerical results of one eigenvector corresponding to the second eigenvalue. In the fourth and sixth columns in Table 5.1 we observe a cubic convergence approximately, since we use the lowest order Raviart Thomas boundary element space.

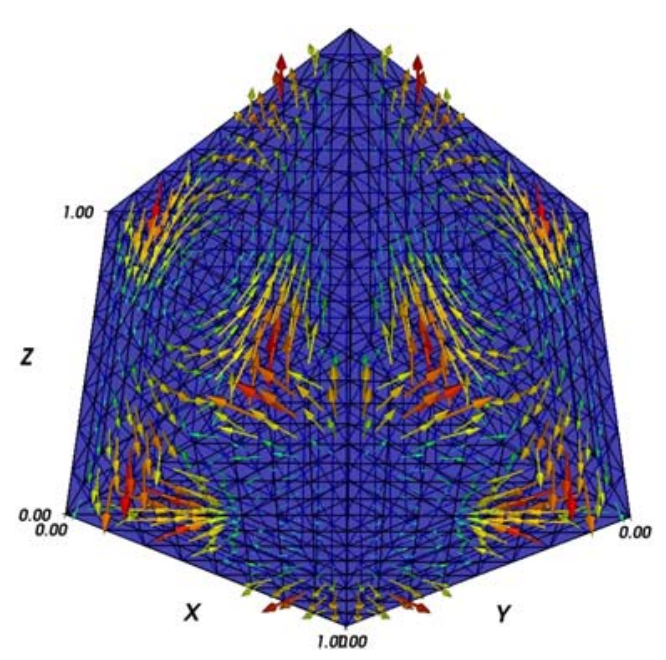

(a) Dirichlet trace of one eigenvector corresponding to the first eigenvalue on $\Gamma^{\text {per }}$

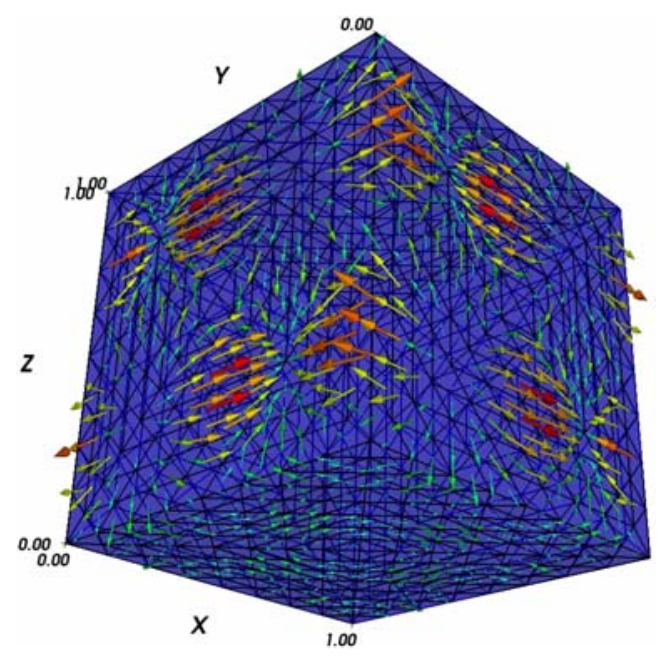

(c) Dirichlet trace of one eigenvector corresponding to the second eigenvalue on $\Gamma^{\text {per }}$

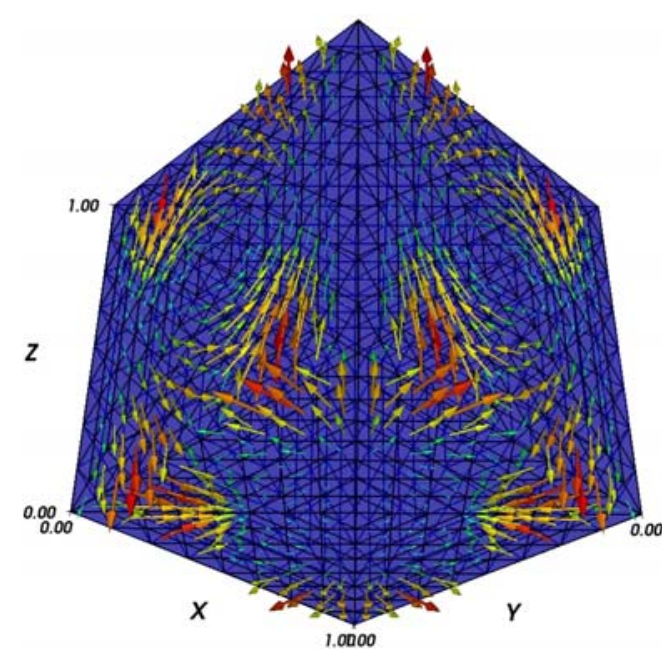

(b) Neumann trace of one eigenvector corresponding to the first eigenvalue on $\Gamma^{\text {per }}$

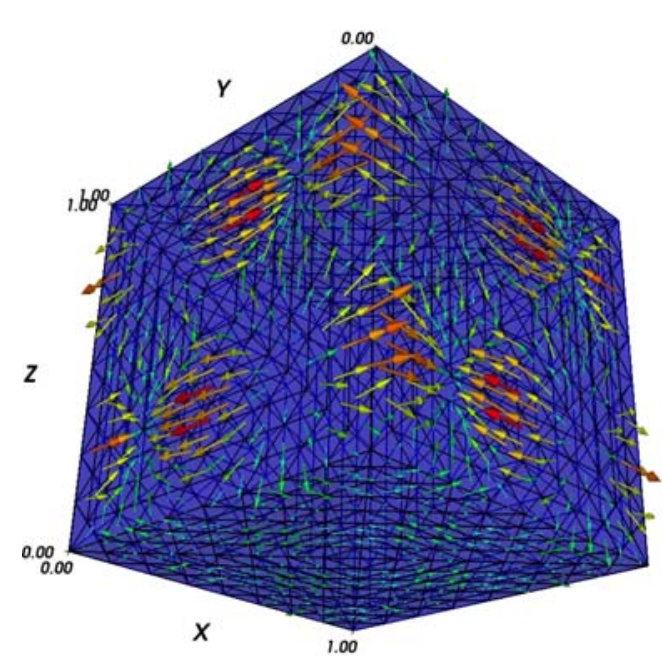

(d) Neumann trace of one eigenvector corresponding to the second eigenvalue on $\Gamma^{p e r}$

Figure 5.3 First eigenvector and second eigenvector of the eigenvalue problem for Maxwell's equations with periodic boundary conditions 


\begin{tabular}{|c|c|c|c|c|c|}
\hline \multicolumn{2}{|c|}{} & \multicolumn{2}{|c|}{ first eigenvalue } & \multicolumn{2}{c|}{ second eigenvalue } \\
\hline level & dof & $\left|k_{h}-k\right|$ & $\mathrm{CR}_{k}$ & $\left|k_{h}-k\right|$ & $\mathrm{CR}_{k}$ \\
\hline 2 & 552 & 8.83865 & - & 10.78751 & - \\
\hline 3 & 2256 & 8.88096 & 3.2930 & 10.87471 & 3.5589 \\
\hline$\infty$ & & 8.88577 & & 10.88280 & \\
\hline
\end{tabular}

Table 5.1 Convergence of the first eigenvalue and second eigenvalue of the eigenvalue problem for Maxwell's equations with periodic boundary conditions

\subsection{An inhomogeneous problem with periodic boundary conditions}

In this section we consider an interface eigenvalue problem for (5.1.9) and assume that $\boldsymbol{\alpha}=(0,0,0)$. Quasi-periodic boundary conditions in $(5.1 .9 \mathrm{c})$ and $(5.1 .9 \mathrm{~d})$ are simplified to periodic boundary conditions. The Maxwell's equations (5.1.9) with interface conditions (5.1.10) are changed to

$$
\begin{array}{lr}
\frac{1}{\mu} \nabla \times\left(\frac{1}{\varepsilon} \nabla \times \mathbf{u}\right)=\omega^{2} \mathbf{u} & \forall x \in \Omega^{e x} \bigcup \Omega^{i n}, \\
\nabla \cdot \mathbf{u}=0 & \forall x \in \Omega^{e x} \bigcup \Omega^{i n}, \\
\gamma_{\mathbf{t}}^{e x}\left(\mathbf{u}^{e x}\right)\left(x+\mathbf{e}_{i}\right)=-\gamma_{\mathbf{t}}^{e x}\left(\mathbf{u}^{e x}\right)(x) & \forall x \in \Gamma_{i}, i=1,2,3, \\
\gamma_{\mathbf{N}}^{e x}\left(\mathbf{u}^{e x}\right)\left(x+\mathbf{e}_{i}\right)=-\gamma_{\mathbf{N}}^{e x}\left(\mathbf{u}^{e x}\right)(x) & \forall x \in \Gamma_{i}, i=1,2,3, \\
\gamma_{\mathbf{t}}^{e x}\left(\mathbf{u}^{e x}\right)(x)+\gamma_{\mathbf{t}}^{i n}\left(\mathbf{u}^{i n}\right)(x)=0 & \forall x \in \Gamma^{I}, \\
\beta^{e x} \gamma_{\mathbf{N}}^{e x}\left(\mathbf{u}^{e x}\right)(x)+\beta^{i n} \gamma_{\mathbf{N}}^{i n}\left(\mathbf{u}^{i n}\right)(x)=0 & \forall x \in \Gamma^{I},
\end{array}
$$

where all the notations are the same as in (5.1.9) and (5.1.10).

\subsubsection{Nonlinear solution method}

As in Section 4.2.1 we use domain decomposition methods to solve this interface eigenvalue problem. We define $\boldsymbol{\sigma}^{e x}:=\gamma_{\mathbf{N}}^{e x}\left(\mathbf{u}^{e x}\right), \boldsymbol{\sigma}^{i n}:=\gamma_{\mathbf{N}}^{i n}\left(\mathbf{u}^{i n}\right), \varphi^{e x}:=\gamma_{\mathbf{t}}^{e x}\left(\mathbf{u}^{e x}\right)$ and $\varphi^{i n}:=\gamma_{\mathbf{t}}^{i n}\left(\mathbf{u}^{i n}\right)$. From (1.3.14) we have two Calderon projections for $\left(\boldsymbol{\sigma}^{e x}, \boldsymbol{\varphi}^{e x}\right)$ and $\left(\sigma^{i n}, \varphi^{i n}\right)$, respectively, as

$$
\begin{aligned}
\left(\begin{array}{l}
\boldsymbol{\varphi}^{e x} \\
\boldsymbol{\sigma}^{e x}
\end{array}\right) & =\left(\begin{array}{cc}
\frac{1}{2} I+\mathbf{C}_{k^{e x}}^{\Gamma^{e x}} & \mathbf{S}_{k^{e x}}^{\Gamma^{e x}} \\
\mathbf{S}_{k^{e x}}^{\Gamma^{e x}} & \frac{1}{2} I+\mathbf{C}_{k^{e x}}^{\Gamma^{e x}}
\end{array}\right)\left(\begin{array}{l}
\boldsymbol{\varphi}^{e x} \\
\boldsymbol{\sigma}^{e x}
\end{array}\right), \\
\left(\begin{array}{c}
\boldsymbol{\varphi}^{i n} \\
\boldsymbol{\sigma}^{i n}
\end{array}\right) & =\left(\begin{array}{cc}
\frac{1}{2} I+\mathbf{C}_{k^{i n}}^{\Gamma^{i n}} & \mathbf{S}_{k^{i n}}^{\Gamma^{i n}} \\
\mathbf{S}_{k^{i n}}^{\Gamma^{i n}} & \frac{1}{2} I+\mathbf{C}_{k^{i n}}^{\Gamma^{i n}}
\end{array}\right)\left(\begin{array}{l}
\boldsymbol{\varphi}^{i n} \\
\boldsymbol{\sigma}^{i n}
\end{array}\right),
\end{aligned}
$$

where $k^{e x}=\sqrt{\alpha^{e x}} k, k^{i n}=\sqrt{\alpha^{i n}} k$, and $\mathbf{S}_{k^{e x}}^{\Gamma^{e x}}, \mathbf{C}_{k^{e x}}^{\Gamma^{e x}}, \mathbf{S}_{k^{i n}}^{\Gamma^{i n}}, \mathbf{C}_{k^{i n}}^{\Gamma^{i n}}$ are boundary integral operators on $\Gamma^{e x}$ and $\Gamma^{i n}$ with the definitions in Section 1.3.3. $\alpha^{e x}=\varepsilon^{e x} \mu^{e x}$ and $\alpha^{i n}=\varepsilon^{i n} \mu^{i n}$. From the interface condition (5.3.1e) we have $\varphi^{e x}=-\varphi^{i n}$ on $\Gamma^{I}$. We define $\varphi^{I}$ as $\varphi^{I}=\varphi^{e x}=-\varphi^{i n}$ on $\Gamma^{I}$ and $\varphi^{I}=0$ on $\Gamma$. We use the second equations of 
(5.3.2a) and (5.3.2b) in (5.3.1f) and get

$$
\begin{aligned}
\beta^{e x}\left(\mathbf{S}_{k^{e x}}^{\Gamma^{e x}}\left(\boldsymbol{\varphi}^{e x}\right)+\right. & \left.\left(\frac{1}{2} I+\mathbf{C}_{k^{e x}}^{\Gamma^{e x}}\right)\left(\boldsymbol{\sigma}^{e x}\right)\right) \\
& +\beta^{i n}\left(\mathbf{S}_{k^{i n}}^{\Gamma^{i n}}\left(\boldsymbol{\varphi}^{i n}\right)+\left(\frac{1}{2} I+\mathbf{C}_{k^{i n}}^{\Gamma^{i n}}\right)\left(\boldsymbol{\sigma}^{i n}\right)\right)=0
\end{aligned}
$$

and by using $\varphi^{I}$ we get

$$
\begin{aligned}
& \left(\beta^{e x} \mathbf{S}_{k^{e x}}^{\Gamma^{I}}+\beta^{i n} \mathbf{S}_{k^{i n}}^{\Gamma^{I}}\right)\left(\boldsymbol{\varphi}^{I}\right)(x)+\beta^{e x} \mathbf{S}_{k^{e x}}^{\Gamma}\left(\left.\boldsymbol{\varphi}^{e x}\right|_{\Gamma}\right)(x) \\
& +\beta^{e x}\left(\frac{1}{2} I+\mathbf{C}_{k^{e x}}^{\Gamma^{I}}\right)\left(\left.\boldsymbol{\sigma}^{e x}\right|_{\Gamma^{I}}\right)(x)+\beta^{e x} \mathbf{C}_{k^{e x}}^{\Gamma}\left(\left.\boldsymbol{\sigma}^{e x}\right|_{\Gamma}\right)(x) \\
& +\beta^{i n}\left(\frac{1}{2} I+\mathbf{C}_{k^{i n}}^{\Gamma^{i n}}\right)\left(\boldsymbol{\sigma}^{i n}\right)(x)=0
\end{aligned}
$$

By using the second equation in (5.3.2a) on $\Gamma$ we get

$$
\mathbf{S}_{k^{e x}}^{\Gamma^{e x}}\left(\boldsymbol{\varphi}^{e x}\right)+\left(-\frac{1}{2} I+\mathbf{C}_{k^{e x}}^{\Gamma^{e x}}\right)\left(\boldsymbol{\sigma}^{e x}\right)=0
$$

and by using $\varphi^{I}$ we get

$$
\begin{aligned}
\mathbf{S}_{k^{e x}}^{\Gamma^{I}}\left(\boldsymbol{\varphi}^{I}\right)(x)+\mathbf{S}_{k^{e x}}^{\Gamma}\left(\left.\boldsymbol{\varphi}^{e x}\right|_{\Gamma}\right)(x)+\mathbf{C}_{k^{e x}}^{\Gamma^{I}}\left(\left.\boldsymbol{\sigma}^{e x}\right|_{\Gamma^{I}}\right)(x) & \\
& +\left(-\frac{1}{2} I+\mathbf{C}_{k^{e x}}^{\Gamma}\right)\left(\left.\boldsymbol{\sigma}^{e x}\right|_{\Gamma}\right)(x)=0
\end{aligned}
$$

By using the first equations in (5.3.2a) and (5.3.2b) we get

$$
\begin{aligned}
& \left(-\frac{1}{2} I+\mathbf{C}_{k^{e x}}^{\Gamma^{e x}}\right)\left(\boldsymbol{\varphi}^{e x}\right)+\mathbf{S}_{k^{e x}}^{\Gamma^{e x}}\left(\boldsymbol{\sigma}^{e x}\right)=0 \\
& \left(-\frac{1}{2} I+\mathbf{C}_{k^{i n}}^{\Gamma^{i n}}\right)\left(\boldsymbol{\varphi}^{i n}\right)+\mathbf{S}_{k^{i n}}^{\Gamma^{i n}}\left(\boldsymbol{\sigma}^{i n}\right)=0
\end{aligned}
$$

and by using $\varphi^{I}$ we get

$$
\begin{aligned}
& \left(-\frac{1}{2} I+\mathbf{C}_{k^{e x}}^{\Gamma^{I}}\right)\left(\boldsymbol{\varphi}^{I}\right)(x)+\mathbf{C}_{k^{e x}}^{\Gamma}\left(\left.\boldsymbol{\varphi}^{e x}\right|_{\Gamma}\right)(x) \\
& +\mathbf{S}_{k^{e x}}^{\Gamma^{I}}\left(\left.\boldsymbol{\sigma}^{e x}\right|_{\Gamma^{I}}\right)(x)+\mathbf{S}_{k^{e x}}^{\Gamma}\left(\left.\boldsymbol{\sigma}^{e x}\right|_{\Gamma}\right)(x)=0 \\
& \mathbf{C}_{k^{e x}}^{\Gamma^{I}}\left(\boldsymbol{\varphi}^{I}\right)(x)+\left(-\frac{1}{2} I+\mathbf{C}_{k^{e x}}^{\Gamma}\right)\left(\left.\boldsymbol{\varphi}^{e x}\right|_{\Gamma}\right)(x) \\
& +\mathbf{S}_{k^{e x}}^{\Gamma^{I}}\left(\left.\boldsymbol{\sigma}^{e x}\right|_{\Gamma^{I}}\right)(x)+\mathbf{S}_{k^{e x}}^{\Gamma}\left(\left.\boldsymbol{\sigma}^{e x}\right|_{\Gamma}\right)(x)=0 \\
& \left(\frac{1}{2} I-\mathbf{C}_{k^{i n}}^{\Gamma^{i n}}\right)\left(\boldsymbol{\varphi}^{I}\right)(x)+\mathbf{S}_{k^{i n}}^{\Gamma^{i n}}\left(\boldsymbol{\sigma}^{i n}\right)(x)=0 \quad \text { a.e. } x \in \Gamma^{i n} .
\end{aligned}
$$


The combination of (5.3.3), (5.3.4), (5.3.5), (5.3.6) and (5.3.7) is the system of equations in distributional sense. By using their left hand side, we define five operators as

$$
\begin{aligned}
\mathbf{F}_{1}: & \mathbf{W}^{-1 / 2}\left(\Gamma^{I}\right) \times \mathbf{W}^{-1 / 2}(\Gamma) \times \mathbf{W}^{-1 / 2}\left(\Gamma^{I}\right) \times \mathbf{W}^{-1 / 2}(\Gamma) \times \mathbf{W}^{-1 / 2}\left(\Gamma^{i n}\right) \times \mathbb{R} \\
& \rightarrow \mathbf{W}^{-1 / 2}\left(\Gamma^{I}\right), \\
\mathbf{F}_{2}: & \mathbf{W}^{-1 / 2}\left(\Gamma^{I}\right) \times \mathbf{W}^{-1 / 2}(\Gamma) \times \mathbf{W}^{-1 / 2}\left(\Gamma^{I}\right) \times \mathbf{W}^{-1 / 2}(\Gamma) \times \mathbb{R} \rightarrow \mathbf{W}^{-1 / 2}(\Gamma), \\
\mathbf{F}_{3}: & \mathbf{W}^{-1 / 2}\left(\Gamma^{I}\right) \times \mathbf{W}^{-1 / 2}(\Gamma) \times \mathbf{W}^{-1 / 2}\left(\Gamma^{I}\right) \times \mathbf{W}^{-1 / 2}(\Gamma) \times \mathbb{R} \rightarrow \mathbf{W}^{-1 / 2}\left(\Gamma^{I}\right), \\
\mathbf{F}_{4}: & \mathbf{W}^{-1 / 2}\left(\Gamma^{I}\right) \times \mathbf{W}^{-1 / 2}(\Gamma) \times \mathbf{W}^{-1 / 2}\left(\Gamma^{I}\right) \times \mathbf{W}^{-1 / 2}(\Gamma) \times \mathbb{R} \rightarrow \mathbf{W}^{-1 / 2}(\Gamma), \\
\mathbf{F}_{5}: & \mathbf{W}^{-1 / 2}\left(\Gamma^{i n}\right) \times \mathbf{W}^{-1 / 2}\left(\Gamma^{i n}\right) \times \mathbb{R} \rightarrow \mathbf{W}^{-1 / 2}\left(\Gamma^{i n}\right) .
\end{aligned}
$$

The variational formulation for this system of equations is to find $k$ with a nontrivial solution $\left(\boldsymbol{\varphi}^{I},\left.\boldsymbol{\varphi}^{e x}\right|_{\Gamma},\left.\boldsymbol{\sigma}^{e x}\right|_{\Gamma^{I}},\left.\boldsymbol{\sigma}^{e x}\right|_{\Gamma}, \boldsymbol{\sigma}^{i n}, k\right) \in \mathbf{W}^{-1 / 2}\left(\Gamma^{I}\right) \times \mathbf{W}^{-1 / 2}(\Gamma) \times \mathbf{W}^{-1 / 2}\left(\Gamma^{I}\right) \times$ $\mathbf{W}^{-1 / 2}(\Gamma) \times \mathbf{W}^{-1 / 2}\left(\Gamma^{i n}\right)$ such that

$$
\begin{aligned}
& \left\langle\mathbf{F}_{1}\left(\boldsymbol{\varphi}^{I},\left.\boldsymbol{\varphi}^{e x}\right|_{\Gamma},\left.\boldsymbol{\sigma}^{e x}\right|_{\Gamma^{I}},\left.\boldsymbol{\sigma}^{e x}\right|_{\Gamma}, \boldsymbol{\sigma}^{i n}, k\right), \boldsymbol{v}^{1}\right\rangle_{\tau, \Gamma^{I}}=0, \\
& \left\langle\mathbf{F}_{2}\left(\boldsymbol{\varphi}^{I},\left.\boldsymbol{\varphi}^{e x}\right|_{\Gamma},\left.\boldsymbol{\sigma}^{e x}\right|_{\Gamma^{I}},\left.\boldsymbol{\sigma}^{e x}\right|_{\Gamma}, k\right), \boldsymbol{v}^{2}\right\rangle_{\tau, \Gamma}=0 \\
& \left\langle\mathbf{F}_{3}\left(\boldsymbol{\varphi}^{I},\left.\boldsymbol{\varphi}^{e x}\right|_{\Gamma},\left.\boldsymbol{\sigma}^{e x}\right|_{\Gamma^{I}},\left.\boldsymbol{\sigma}^{e x}\right|_{\Gamma}, k\right), \boldsymbol{v}^{3}\right\rangle_{\tau, \Gamma^{I}}=0, \\
& \left\langle\mathbf{F}_{4}\left(\boldsymbol{\varphi}^{I},\left.\boldsymbol{\varphi}^{e x}\right|_{\Gamma},\left.\boldsymbol{\sigma}^{e x}\right|_{\Gamma^{I}},\left.\boldsymbol{\sigma}^{e x}\right|_{\Gamma}, k\right), \boldsymbol{v}^{3}\right\rangle_{\tau, \Gamma}=0, \\
& \left\langle\mathbf{F}_{5}\left(\boldsymbol{\varphi}^{I}, \boldsymbol{\sigma}^{i n}, k\right), \boldsymbol{v}^{5}\right\rangle_{\tau, \Gamma^{i n}}=0
\end{aligned}
$$

for all $\left(\boldsymbol{v}^{1}, \boldsymbol{v}^{2}, \boldsymbol{v}^{3}, \boldsymbol{v}^{4}, \boldsymbol{v}^{5}\right) \in \mathbf{W}^{-1 / 2}\left(\Gamma^{I}\right) \times \mathbf{W}^{-1 / 2}(\Gamma) \times \mathbf{W}^{-1 / 2}\left(\Gamma^{I}\right) \times \mathbf{W}^{-1 / 2}(\Gamma) \times$ $\mathbf{W}^{-1 / 2}\left(\Gamma^{i n}\right)$, and $\left.\varphi^{e x}\right|_{\Gamma}$ and $\left.\boldsymbol{\sigma}^{e x}\right|_{\Gamma}$ satisfy the periodic boundary conditions (5.3.1c) and (5.3.1d).

The discretization for the variational formulation (5.3.8) is to find $k_{h}$ with a nontrivial solution $\left(\boldsymbol{\varphi}_{h}^{I},\left.\boldsymbol{\varphi}_{h}^{e x}\right|_{\Gamma_{h}},\left.\boldsymbol{\sigma}_{h}^{e x}\right|_{\Gamma_{h}^{I}},\left.\boldsymbol{\sigma}_{h}^{e x}\right|_{\Gamma_{h}}, \boldsymbol{\sigma}_{h}^{i n}, k_{h}\right) \in \mathbf{W}_{h}^{-1 / 2}\left(\Gamma_{h}^{I}\right) \times \mathbf{W}_{h}^{-1 / 2}\left(\Gamma_{h}\right) \times \mathbf{W}_{h}^{-1 / 2}\left(\Gamma_{h}^{I}\right) \times$ $\mathbf{W}_{h}^{-1 / 2}\left(\Gamma_{h}\right) \times \mathbf{W}_{h}^{-1 / 2}\left(\Gamma_{h}^{i n}\right)$ such that

$$
\begin{aligned}
& \left\langle\mathbf{F}_{1}\left(\boldsymbol{\varphi}_{h}^{I},\left.\boldsymbol{\varphi}_{h}^{e x}\right|_{\Gamma_{h}},\left.\boldsymbol{\sigma}_{h}^{e x}\right|_{\Gamma_{h}^{I}},\left.\boldsymbol{\sigma}_{h}^{e x}\right|_{\Gamma_{h}}, \boldsymbol{\sigma}_{h}^{i n}, k_{h}\right), \boldsymbol{v}_{h}^{1}\right\rangle_{\tau, \Gamma^{I}}=0, \\
& \left\langle\mathbf{F}_{2}\left(\boldsymbol{\varphi}_{h}^{I},\left.\boldsymbol{\varphi}_{h}^{e x}\right|_{\Gamma_{h}},\left.\boldsymbol{\sigma}_{h}^{e x}\right|_{\Gamma_{h}^{I}},\left.\boldsymbol{\sigma}_{h}^{e x}\right|_{\Gamma_{h}}, k_{h}\right), \boldsymbol{v}_{h}^{2}\right\rangle_{\tau, \Gamma}=0, \\
& \left\langle\mathbf{F}_{3}\left(\boldsymbol{\varphi}_{h}^{I},\left.\boldsymbol{\varphi}_{h}^{e x}\right|_{\Gamma_{h}},\left.\boldsymbol{\sigma}_{h}^{e x}\right|_{\Gamma_{h}^{I}},\left.\boldsymbol{\sigma}_{h}^{e x}\right|_{\Gamma_{h}}, k_{h}\right), \boldsymbol{v}_{h}^{3}\right\rangle_{\tau, \Gamma^{I}}=0, \\
& \left\langle\mathbf{F}_{4}\left(\boldsymbol{\varphi}_{h}^{I},\left.\boldsymbol{\varphi}_{h}^{e x}\right|_{\Gamma_{h}},\left.\boldsymbol{\sigma}_{h}^{e x}\right|_{\Gamma_{h}^{I}},\left.\boldsymbol{\sigma}_{h}^{e x}\right|_{\Gamma_{h}}, k_{h}\right), \boldsymbol{v}_{h}^{4}\right\rangle_{\tau, \Gamma}=0, \\
& \left\langle\mathbf{F}_{5}\left(\boldsymbol{\varphi}_{h}^{I}, \boldsymbol{\sigma}_{h}^{i n}, k_{h}\right), \boldsymbol{v}_{h}^{5}\right\rangle_{\tau, \Gamma^{i n}}=0,
\end{aligned}
$$

for all $\left(\boldsymbol{v}_{h}^{1}, \boldsymbol{v}_{h}^{2}, \boldsymbol{v}_{h}^{3}, \boldsymbol{v}_{h}^{4}, \boldsymbol{v}_{h}^{5}\right) \in \mathbf{W}_{h}^{-1 / 2}\left(\Gamma_{h}^{I}\right) \times \mathbf{W}_{h}^{-1 / 2}\left(\Gamma_{h}\right) \times \mathbf{W}_{h}^{-1 / 2}\left(\Gamma_{h}^{I}\right) \times \mathbf{W}_{h}^{-1 / 2}\left(\Gamma_{h}\right) \times$ $\mathbf{W}_{h}^{-1 / 2}\left(\Gamma_{h}^{i n}\right)$ and $\left.\boldsymbol{\varphi}_{h}^{e x}\right|_{\Gamma_{h}}$ and $\left.\boldsymbol{\sigma}_{h}^{e x}\right|_{\Gamma_{h}}$ satisfy the periodic boundary conditions.

Let $\left\{\boldsymbol{\Phi}_{i}^{I}\right\}_{i=1}^{N^{I}}$ be a basis of $\mathbf{W}_{h}^{-1 / 2}\left(\Gamma_{h}^{I}\right)$ and $\left\{\boldsymbol{\Phi}_{i}^{\Gamma}\right\}_{i=1}^{N^{\Gamma}}$ be a basis of $\mathbf{W}_{h}^{-1 / 2}\left(\Gamma_{h}\right)$. We use $\boldsymbol{\varphi}_{h}^{I}=\sum_{j=1}^{N^{I}} \xi_{j}^{1} \boldsymbol{\Phi}_{j}^{I},\left.\boldsymbol{\varphi}_{h}^{e x}\right|_{\Gamma_{h}}=\sum_{j=1}^{N^{\Gamma}} \xi_{j}^{2} \boldsymbol{\Phi}_{j}^{\Gamma},\left.\boldsymbol{\sigma}_{h}^{e x}\right|_{\Gamma_{h}^{I}}=\sum_{j=1}^{N^{I}} \xi_{j}^{3} \boldsymbol{\Phi}_{j}^{I},\left.\boldsymbol{\sigma}_{h}^{e x}\right|_{\Gamma_{h}}=\sum_{j=1}^{N^{\Gamma}} \xi_{j}^{4} \boldsymbol{\Phi}_{j}^{\Gamma}$ 
and $\boldsymbol{\sigma}_{h}^{i n}=\sum_{j=1}^{N^{I}} \xi_{j}^{5} \boldsymbol{\Phi}_{j}^{I}$ in (5.3.9) and get a nonlinear system

$$
\operatorname{Re} A\left(k_{h}\right) \xi:=\operatorname{Re}\left(\begin{array}{ccccc}
A_{11}\left(k_{h}\right) & A_{12}\left(k_{h}\right) & A_{13}\left(k_{h}\right) & A_{14}\left(k_{h}\right) & A_{15}\left(k_{h}\right) \\
A_{21}\left(k_{h}\right) & A_{22}\left(k_{h}\right) & A_{23}\left(k_{h}\right) & A_{24}\left(k_{h}\right) & 0 \\
A_{31}\left(k_{h}\right) & A_{32}\left(k_{h}\right) & A_{33}\left(k_{h}\right) & A_{34}\left(k_{h}\right) & 0 \\
A_{41}\left(k_{h}\right) & A_{42}\left(k_{h}\right) & A_{43}\left(k_{h}\right) & A_{44}\left(k_{h}\right) & 0 \\
A_{51}\left(k_{h}\right) & 0 & 0 & 0 & A_{55}\left(k_{h}\right)
\end{array}\right)\left(\begin{array}{l}
\xi^{1} \\
\xi^{2} \\
\xi^{3} \\
\xi^{4} \\
\xi^{5}
\end{array}\right)=0
$$

where $\xi_{2}$ and $\xi_{4}$ satisfy the periodic boundary conditions. It is easy to check that $A$ is a symmetric matrix. The calculation of the elements in $A$ is similar with Section 3.3.1 and Section 4.2.1. The definition of $\mathbf{W}_{h}^{-1 / 2}\left(\Gamma_{h}^{p e r}\right)$ is the same as in Section 5.2.1. By the periodic boundary conditions we can define a mapping $B$ such that

$$
\xi^{2}=B \xi_{p e r}^{2} \quad \text { and } \quad \xi^{4}=B \xi_{p e r}^{4} .
$$

where $\xi_{p e r}^{2}$ and $\xi_{\text {per }}^{4}$ are the coefficients of the testing functions defined on $\Gamma_{h}^{p e r}$. We use (5.3.11) in (5.3.10) and get a system of equations

$$
\begin{aligned}
& A^{\text {per }}\left(k_{h}\right) \xi^{\text {per }}:= \\
& \operatorname{Re}\left(\begin{array}{ccccc}
A_{11}\left(k_{h}\right) & A_{12}\left(k_{h}\right) B & A_{13}\left(k_{h}\right) & A_{14}\left(k_{h}\right) B & A_{15}\left(k_{h}\right) \\
B^{T} A_{21}\left(k_{h}\right) & B^{T} A_{22}\left(k_{h}\right) B & B^{T} A_{23}\left(k_{h}\right) & B^{T} A_{24}\left(k_{h}\right) B & 0 \\
A_{31}\left(k_{h}\right) & A_{32}\left(k_{h}\right) B & A_{33}\left(k_{h}\right) & A_{34}\left(k_{h}\right) B & 0 \\
B^{T} A_{41}\left(k_{h}\right) & B^{T} A_{42}\left(k_{h}\right) B & B^{T} A_{43}\left(k_{h}\right) & B^{T} A_{44}\left(k_{h}\right) B & 0 \\
A_{51}\left(k_{h}\right) & 0 & 0 & 0 & A_{55}\left(k_{h}\right)
\end{array}\right)\left(\begin{array}{c}
\xi^{1} \\
\xi_{\text {per }}^{2} \\
\xi^{3} \\
\xi_{\text {per }}^{4} \\
\xi^{5}
\end{array}\right) \\
& =0 .
\end{aligned}
$$

To avoid a trivial solution, we include a suitable normalization for $\xi^{\text {per }}$ by $\left\|\xi^{\text {per }}\right\|_{l^{2}}=1$. Then the nonlinear equations for $\left(\xi^{p e r}, k_{h}\right)$ is

$$
\begin{aligned}
A^{\text {per }}\left(k_{h}\right) \xi^{\text {per }} & =0 \\
\left\|\xi^{\text {per }}\right\|_{l^{2}}-1 & =0 .
\end{aligned}
$$

We continue to use the Newton method to solve (5.3.13). The Frechet derivative is derived as the following.

$$
\begin{gathered}
\lim _{h \rightarrow 0} \frac{A^{\text {per }}\left(k+h k^{\prime}\right)\left(\xi^{p e r}+h \xi^{p e r \prime}\right)-A^{\text {per }}(k)\left(\xi^{p e r}\right)}{h}=A^{\text {per }}(k) \xi^{\text {per } \prime}+k^{\prime} C^{\text {per }}(k) \xi^{\text {per }} \\
\lim _{h \rightarrow 0} \frac{\left\|\xi^{\text {per }}+h \xi^{\text {per }}\right\|_{l^{2}}^{2}-\left\|\xi^{\text {per }}\right\|_{l^{2}}^{2}}{h}=2\left(\xi^{\text {per }}, \xi^{\text {per } \prime}\right)_{l^{2}} .
\end{gathered}
$$

\subsubsection{Numerical tests}

We consider the first and second eigenvalues of the interface eigenvalue problem with periodic boundary conditions with corresponding eigenvectors on the boundary of a unit 
cube $(0,1)^{3}$. We assume that $\Omega^{i n}=\left(\frac{1}{3}, \frac{2}{3}\right)^{3}, \Omega^{e x}=(0,1)^{3} \backslash \overline{\Omega^{i n}}, \varepsilon^{i n}=\varepsilon^{e x}=1$ and $\mu^{i n}=\mu^{e x}=1$. An analytical solution used for the test is the same as in Section 5.2.2. The eigenvalue is given by $k=2 \pi \sqrt{m_{1}^{2}+m_{2}^{2}+m_{3}^{2}}$ with $m_{1}, m_{2}, m_{3} \in \mathbb{N}^{0}$. In Table 5.2 the first column is the level of meshes and the second column is the number of degrees of freedom, the third and fifth columns are the numerical results for the first and second eigenvalues and the fourth and sixth columns are the rate of convergence. Fig. 5.4a, 5.4b and $5.4 \mathrm{c}$ are the numerical results of one eigenvector corresponding to the first eigenvalue and Fig. 5.5a, 5.5b and 5.5c are the numerical results of one eigenvector corresponding to the second eigenvalue. In the fourth and sixth columns in Table 5.2 we observe a cubic convergence approximately, since we use the lowest order Raviart Thomas boundary element space.

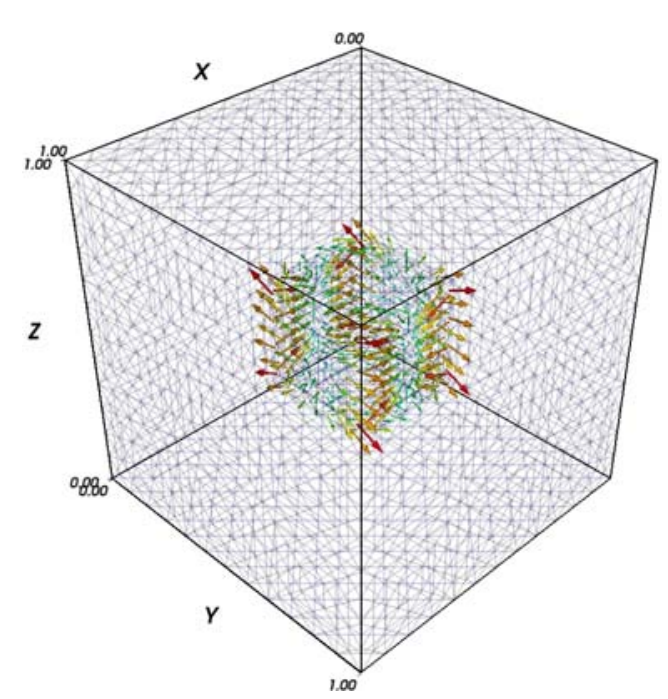

(a) $\varphi^{e x}$ corresponding to the first eigenvalue

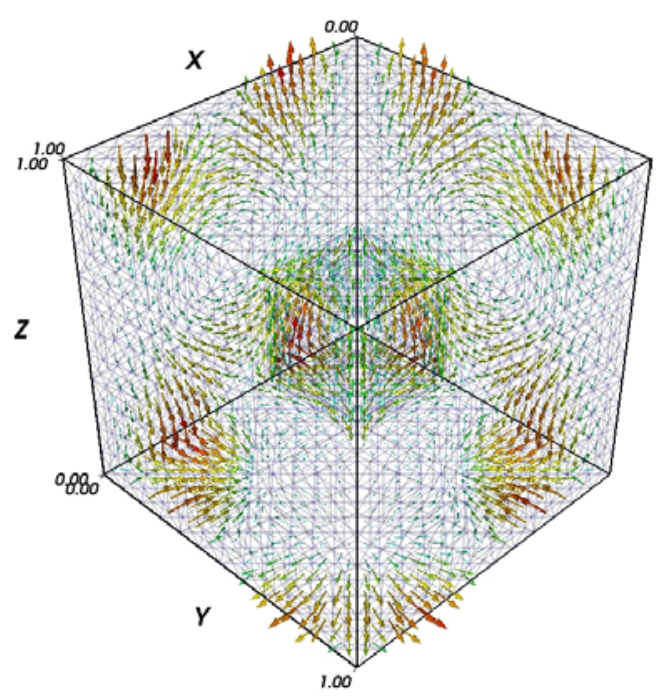

(b) $\sigma^{e x}$ corresponding to the first eigenvalue

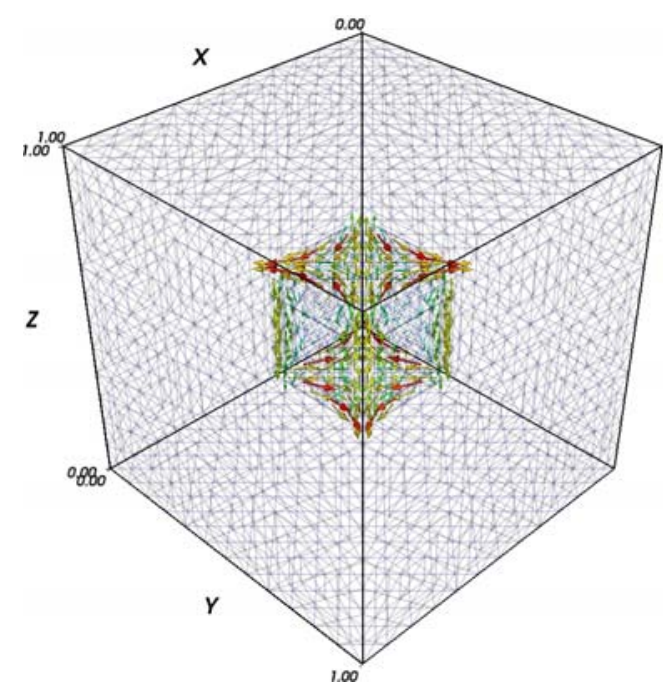

(c) $\sigma^{i n}$ corresponding to the first eigenvalue

Figure 5.4 First eigenvector of the interface eigenvalue problem for Maxwell's equations with periodic boundary conditions 


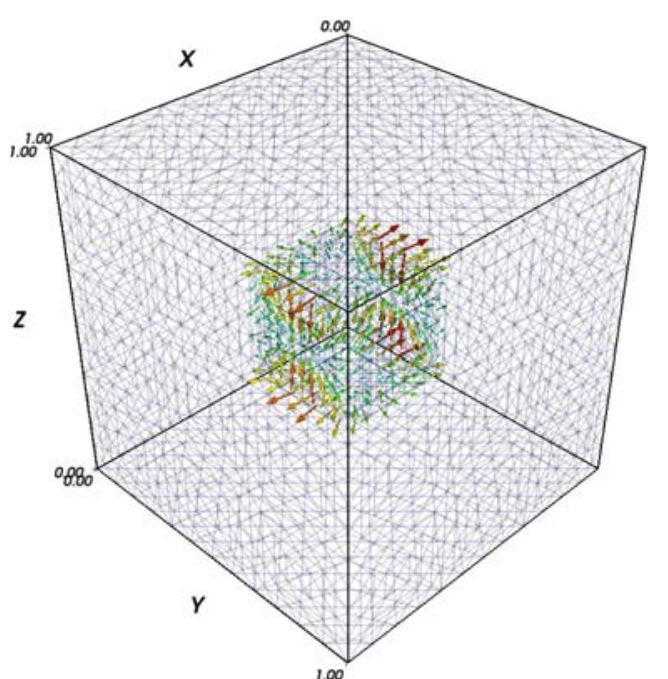

(a) $\varphi^{e x}$ of one eigenvector corresponding to the second eigenvalue

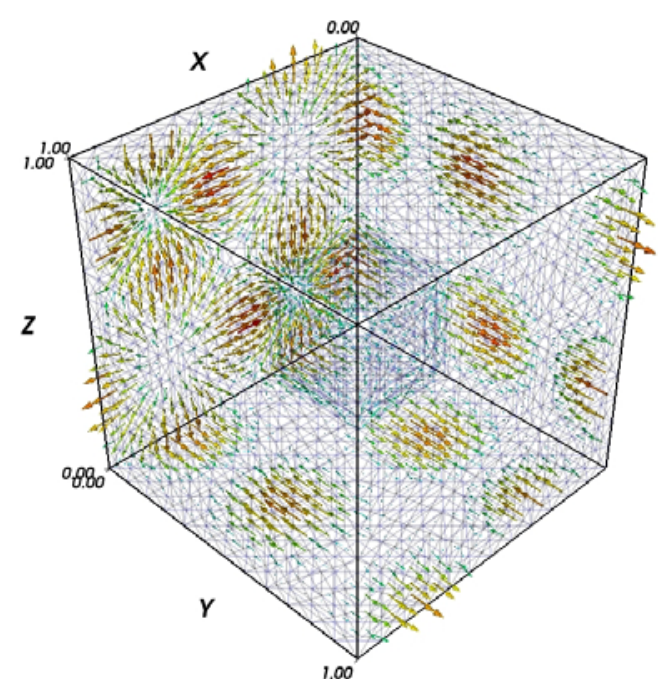

(b) $\sigma^{e x}$ of one eigenvector corresponding to the second eigenvalue

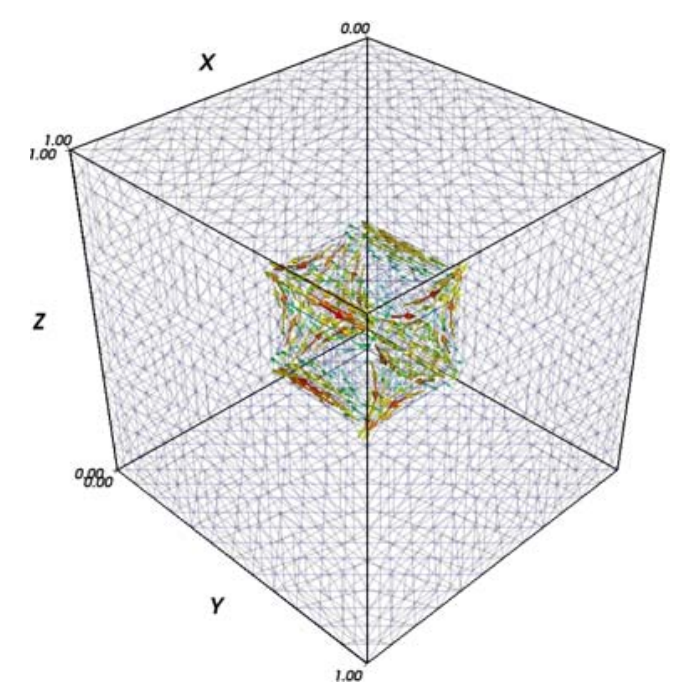

(c) $\sigma^{i n}$ of one eigenvector corresponding to the second eigenvalue

Figure 5.5 Second eigenvector of the interface eigenvalue problem for Maxwell's equations with periodic boundary conditions

\subsection{Comparison of BEMs and FEMs}

In this section we use Galerkin-BEMs to calculate the band structure of photonic crystals and compare the accuracy of our results with FEMs. The numerical results of FEMs are from the $\mathrm{PhD}$ work of Dr. A. Bulovyatov [14]. In the first numerical test we solve the interface eigenvalue problem with quasi-periodic boundary conditions in a homogeneous medium as a test and an analytical solution is known. In the second numerical example we solve the same problem in an inhomogeneous medium without a 


\begin{tabular}{|c|c|c|c|c|c|}
\hline \multicolumn{2}{|l|}{} & \multicolumn{2}{c|}{ first eigenvalue } & \multicolumn{2}{c|}{ second eigenvalue } \\
\hline level & dof & Error & CR & Error & CR \\
\hline 0 & 415 & 8.66876 & - & 10.46234 & - \\
\hline 1 & 1693 & 8.86738 & 3.5608 & 10.85214 & 3.7778 \\
\hline 2 & 6841 & 8.88392 & 3.3185 & 10.87987 & 3.3906 \\
\hline$\infty$ & & 8.88577 & & 10.88280 & \\
\hline
\end{tabular}

Table 5.2 Convergence of the first eigenvalue and second eigenvalue of the interface eigenvalue problem for Maxwell's equations with periodic boundary conditions

given solution which is compared with FEMs.

\subsubsection{Numerical tests}

We assume that $\Omega=(0,1)^{3}, \Omega^{i n}=(1 / 3,2 / 3)^{3}, \Omega^{e x}=(0,1)^{3} \backslash \overline{\Omega^{i n}}$ and $\mu^{e x}=\mu^{i n}=$ $\varepsilon^{e x}=\varepsilon^{i n}=1$. The problem is defined by

$$
\begin{array}{lr}
\nabla \times \nabla \times \mathbf{u}^{i n}(x)=k^{2} \mathbf{u}^{i n}(x) & \forall x \in \Omega^{i n}, \\
\nabla \cdot \mathbf{u}^{i n}(x)=0 & \forall x \in \Omega^{i n}, \\
\nabla \times \nabla \times \mathbf{u}^{e x}(x)=k^{2} \mathbf{u}^{e x}(x) & \forall x \in \Omega^{e x}, \\
\nabla \cdot \mathbf{u}^{e x}(x)=0 & \forall x \in \Omega^{e x}, \\
\gamma_{\mathbf{t}}^{e x}\left(\mathbf{u}^{e x}\right)(x)+\gamma_{\mathbf{t}}^{i n}\left(\mathbf{u}^{i n}\right)(x)=0 & \forall x \in \Gamma^{I}, \\
\gamma_{\mathbf{N}}^{e x}\left(\mathbf{u}^{e x}\right)(x)+\gamma_{\mathbf{N}}^{i n}\left(\mathbf{u}^{i n}\right)(x)=0 & \forall x \in \Gamma^{I}, \\
\gamma_{\mathbf{t}}^{e x}\left(\mathbf{u}^{e x}\right)\left(x+\mathbf{e}_{i}\right)=-\mathrm{e}^{\mathrm{i} \boldsymbol{\alpha} \cdot \mathbf{e}_{i}} \gamma_{\mathbf{t}}^{e x}\left(\mathbf{u}^{e x}\right)(x) & \forall x \in \Gamma_{i}, i=1,2,3, \\
\gamma_{\mathbf{N}}^{e x}\left(\mathbf{u}^{e x}\right)\left(x+\mathbf{e}_{i}\right)=-\mathrm{e}^{\mathrm{i} \boldsymbol{\alpha} \cdot \mathbf{e}_{i}} \gamma_{\mathbf{N}}^{e x}\left(\mathbf{u}^{e x}\right)(x) & \forall x \in \Gamma_{i}, i=1,2,3,
\end{array}
$$

The nonlinear solution method for (5.4.1) is similar with (5.3.1). The only difference is that by using the quasi-periodic boundary conditions (5.1.9c) and (5.1.9d), the mapping $B$ is a complex matrix. Then the nonlinear equation system (5.3.12) is changed to

$$
A^{\text {per }}\left(k_{h}\right) \xi^{\text {per }}:=D^{H} \operatorname{Re} A\left(k_{h}\right) D,
$$

where $A\left(k_{h}\right)$ is given in (5.3.10) and

$$
D:=\left(\begin{array}{ccccc}
I & 0 & 0 & 0 & 0 \\
0 & B & 0 & 0 & 0 \\
0 & 0 & I & 0 & 0 \\
0 & 0 & 0 & B & 0 \\
0 & 0 & 0 & 0 & I
\end{array}\right)
$$

$A^{\text {per }}\left(k_{h}\right)$ is a complex matrix and $\xi^{\text {per }}$ is a complex vector. To avoid a trivial solution, the normalization for a real vector (5.3.13b) is changed to $v^{H} \xi=1$. $v$ is a given complex 
vector and the nonlinear equation system is

$$
\begin{aligned}
A\left(k_{h}\right) \xi & =0, \\
v^{H} \xi-1 & =0 .
\end{aligned}
$$

The Frechet derivative is derived as the following.

$$
\begin{gathered}
\lim _{h \rightarrow 0} \frac{A^{\text {per }}\left(k+h k^{\prime}\right)\left(\xi^{\text {per }}+h \xi^{\text {per' }}\right)-A^{\text {per }}(k)\left(\xi^{\text {per }}\right)}{h}=A^{\text {per }}(k) \xi^{\text {per' }}+k^{\prime} C^{p e r}(k) \xi, \\
\lim _{h \rightarrow 0} \frac{v^{H}\left(\xi^{\text {per }}+h \xi^{\text {per' }}\right)-1-\left(v^{H} \xi^{p e r}-1\right)}{h}=v^{H} \xi^{\text {per' }} .
\end{gathered}
$$

The Newton method is the same with other sections.

An analytical solution used for the test is

$$
\mathbf{u}(x)=\left(\begin{array}{c}
\lambda_{1} \mathbf{u}_{1}(x) \\
\lambda_{2} \mathbf{u}_{2}(x) \\
\lambda_{3} \mathbf{u}_{3}(x)
\end{array}\right)
$$

where

$$
\mathbf{u}_{1}(x)=\mathbf{u}_{2}(x)=\mathbf{u}_{3}(x)=\mathrm{e}^{i \boldsymbol{\alpha}_{1} x_{1}} \mathrm{e}^{i \boldsymbol{\alpha}_{2} x_{2}} \mathrm{e}^{i \boldsymbol{\alpha}_{3} x_{3}} \mathrm{e}^{i 2 m_{1} \pi x_{1}} \mathrm{e}^{i 2 m_{2} \pi x_{2}} \mathrm{e}^{i 2 m_{3} \pi x_{3}} .
$$

for $\boldsymbol{\alpha} \in[-\pi, \pi]^{3}$ and $m_{1}, m_{2}, m_{3} \in \mathbb{Z}^{0}$. From (5.4.1b) and (5.4.1d) we need

$$
\lambda_{1}\left(\boldsymbol{\alpha}_{1}+2 \pi m_{1}\right)+\lambda_{2}\left(\boldsymbol{\alpha}_{2}+2 \pi m_{2}\right)+\lambda_{3}\left(\boldsymbol{\alpha}_{3}+2 \pi m_{3}\right)=0 .
$$

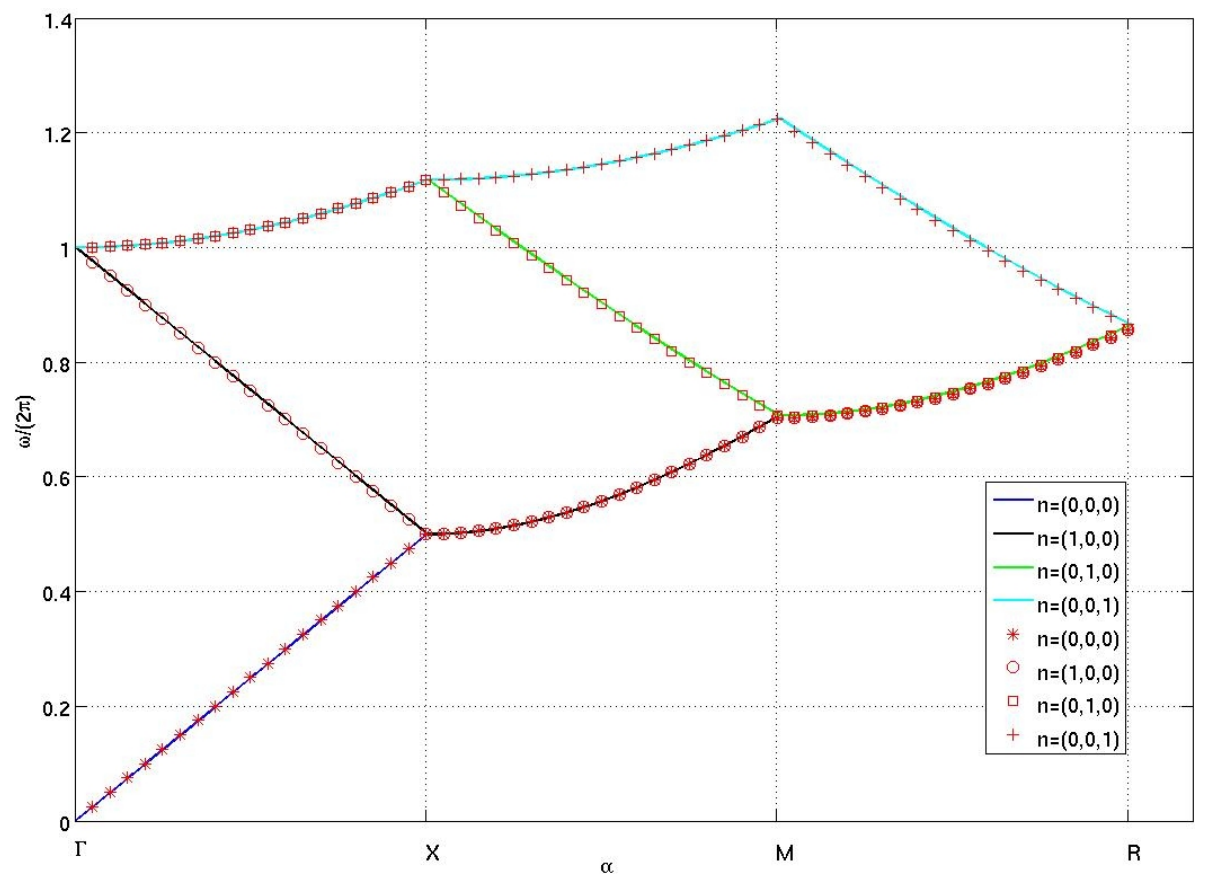

Figure 5.6 Band structure of a homogeneous problem calculated by Galerkin-BEMs 
The eigenvalue is given by

$$
k^{2}=\sum_{i=1}^{3}\left(\boldsymbol{\alpha}_{i}+2 \pi m_{i}\right)^{2} .
$$

The $\mathrm{x}$-axis in Fig. 5.6 is the value of $\boldsymbol{\alpha}$ and we define $\Gamma:=(0,0,0), X:=(-\pi, 0,0)$, $M:=(-\pi,-\pi, 0)$, and $R:=(-\pi,-\pi,-\pi)$. The different eigenvalues for a fixed $\boldsymbol{\alpha}$ are calculated by $m:=\left(m_{1}, m_{2}, m_{3}\right)=(0,0,0), m=(1,0,0), m=(0,1,0)$, and $m=(0,0,1)$. The lines with different colors in Fig. 5.6 are calculated by (5.4.4). The dots with different color in Fig. 5.6 are calculated by Galerkin-BEMs. We observe that the numerical results of Galerkin-BEMs match the analytical solution very well.

\subsubsection{Numerical examples}

We assume that $\Omega=(0,1)^{3}, \Omega^{i n}=(1 / 8,7 / 8)^{3}, \Omega^{e x}=(0,1)^{3} \backslash \bar{\Omega}^{i n}, \mu^{e x}=\mu^{i n}=1$, $\varepsilon^{e x}=13$ and $\varepsilon^{i n}=1$. The problem is defined by

$$
\begin{array}{lr}
\nabla \times \nabla \times \mathbf{h}^{i n}(x)=k^{2} \mathbf{h}^{i n}(x) & \forall x \in \Omega^{i n}, \\
\nabla \cdot \mathbf{h}^{i n}(x)=0 & \forall x \in \Omega^{i n}, \\
\nabla \times \nabla \times \mathbf{h}^{e x}(x)=13 k^{2} \mathbf{h}^{e x}(x) & \forall x \in \Omega^{e x}, \\
\nabla \cdot \mathbf{h}^{e x}(x)=0 & \forall x \in \Omega^{e x}, \\
\gamma_{\mathbf{t}}^{e x}\left(\mathbf{h}^{e x}\right)(x)+\gamma_{\mathbf{t}}^{i n}\left(\mathbf{h}^{i n}\right)(x)=0 & \forall x \in \Gamma^{I}, \\
\left.\gamma_{\mathbf{N}}^{e x}\left(\mathbf{h}^{e x}\right)(x)+\sqrt{13} \gamma_{\mathbf{N}}^{i n} \mathbf{h}^{i n}\right)(x)=0 & \forall x \in \Gamma^{I}, \\
\gamma_{\mathbf{t}}^{e x}\left(\mathbf{h}^{e x}\right)\left(x+\mathbf{e}_{i}\right)=-\mathrm{e}^{\boldsymbol{\alpha} \cdot \mathbf{e}_{i} \mathrm{i}} \gamma_{\mathbf{t}}^{e x}\left(\mathbf{h}^{e x}\right)(x) & \forall x \in \Gamma_{i}, i=1,2,3, \\
\gamma_{\mathbf{N}}^{e x}\left(\mathbf{h}^{e x}\right)\left(x+\mathbf{e}_{i}\right)=-\mathrm{e}^{\boldsymbol{\alpha} \cdot \mathbf{e}_{i} \mathrm{i}} \gamma_{\mathbf{N}}^{e x}\left(\mathbf{h}^{e x}\right)(x) & \forall x \in \Gamma_{i}, i=1,2,3, \\
&
\end{array}
$$

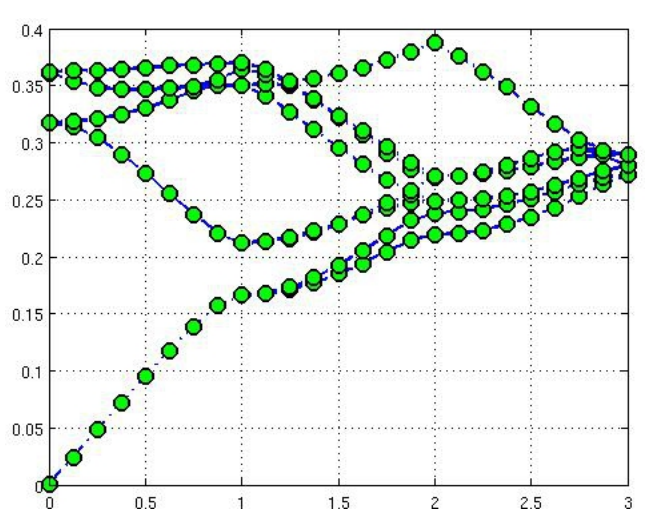

(a) band structure from FEMs

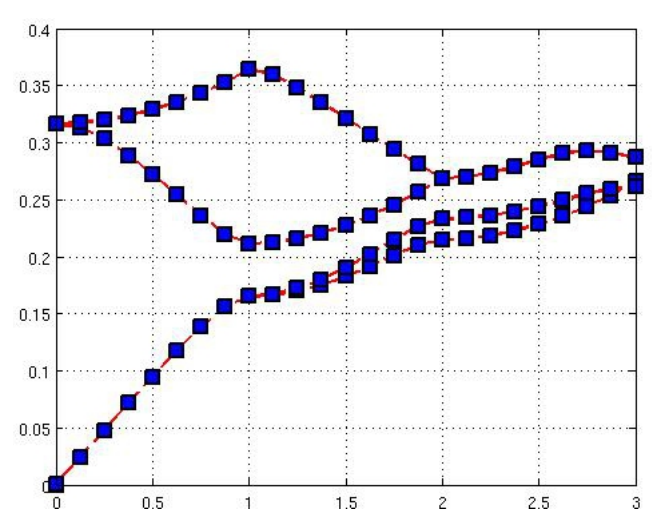

(b) band structure from BEMs

Figure 5.7 Band structure of an inhomogeneous problem solved by Galerkin-BEMs and FEMs 


\begin{tabular}{|c|c|c|c|c|c|}
\hline level & dof & $\Gamma$ & $\left|k^{n}-k^{n-1}\right|$ & $X$ & $\left|k^{n}-k^{n-1}\right|$ \\
\hline 1 & 564 & 1.964717262 & & 1.03892722 & \\
\hline 2 & 2280 & 1.986816725 & 0.0221 & 1.042721944 & 0.0038 \\
\hline 3 & 9168 & 1.98986934 & 0.0031 & 1.043618266 & 0.000896 \\
\hline
\end{tabular}

Table 5.3 Convergence of the eigenvalues calculated by Galerkin-BEMs in band structure

\begin{tabular}{|c|c|c|c|c|c|}
\hline level & dof & $\Gamma$ & $\left|k^{n}-k^{n-1}\right|$ & $X$ & $\left|k^{n}-k^{n-1}\right|$ \\
\hline 3 & 512 & 2.031763 & & 1.052913 & \\
\hline 4 & 4096 & 2.002369 & 0.0294 & 1.046865 & 0.0060 \\
\hline 5 & 32768 & 1.994080 & 0.0083 & 1.044979 & 0.0019 \\
\hline
\end{tabular}

Table 5.4 Convergence of the eigenvalues calculated by finite element methods in band structure

Figure $5.7 \mathrm{a}$ is the band structure of the problem (5.4.5) calculated by finite element methods. Figure $5.7 \mathrm{~b}$ is the band structure of the problem (5.4.5) calculated by GalerkinBEMs. In Tables 5.3 and 5.4 the first column is the level of meshes, the second column is the number of degrees of freedom, the third and fifth columns are the numerical results for one eigenvalue for $\boldsymbol{\alpha}=\Gamma, X$. The other columns are the rate of convergence. From Figure 5.7 and Tables 5.3 and 5.4, we observe that the results of Galerkin-BEMs match the results of finite element methods very well. Nevertheless, there is a problem: if we compare Figure $5.7 \mathrm{a}$ with $5.7 \mathrm{~b}$, we find that we computed only eigenvalues of some band by boundary element methods, since we need a Newton iteration of every single eigenvalue. For finite element methods, block algorithms are available which compute several eigenvalues simultaneously. 


\section{Bibliography}

[1] R.A. Adams and J.J.F. Fournier. Sobolev Spaces. Academic Press, Amsterdam, second edition, 2003.

[2] A. Alonso and A. Valli. Some remarks on the characterization of the space of tangential traces of $\mathbf{H}(\operatorname{rot} ; \Omega)$ and the construction of an extension operator. Manuscripta Math., 89(2):159-178, 1996.

[3] S. Börm, L. Grasedyck, and W. Hackbusch. Hierarchical Matrices (Lecture Notes). Max-Planck Institute, 2003.

[4] S.C. Brenner and L.R. Scott. The Mathematical Theory of Finite Element Methods. Springer, New York, third edition, 2008.

[5] H. Brezis. Functional Analysis, Sobolev Spaces and Partial Differential Equations. Springer, New York, 2010.

[6] A. Buffa. Hodge decompositions on the boundary of a polyhedron: The multiconnected case. Math. Mod. Meth. Appl. Sci., 11:1491-1504, 2001.

[7] A. Buffa. Traces theorems for functional spaces related to maxwell equations: an overview. In Proceedings of GAMM-Workshop, Kiel, 2001.

[8] A. Buffa and S. Christiansen. The electric field integral equation on Lipschitz screens: Definition and numerical approximation. Numer. Mathem., 2002.

[9] A. Buffa and P. Ciarlet. On traces for function spaces related to Maxwell's equations. Part I: An integration by parts formula in Lipschitz polyhedra. Math. Meth. Appl. Sci., 21:9-30, 2001.

[10] A. Buffa and P. Ciarlet. On traces for function spaces related to Maxwell's equations. Part II: Hodge decompositions on the boundary of Lipschitz polyhedra and applications. Math. Meth. Appl. Sci., 21:31-48, 2001.

[11] A. Buffa, M. Costabel, and C. Schwab. Boundary element methods for Maxwell's equations on non-smooth domains. Numer. Mathem., 4/92:679-710, 2002.

[12] A. Buffa, M. Costabel, and D. Sheen. On traces for $\mathbf{H}(\mathbf{c u r l}, \Omega)$ in Lipschitz domains. J. Math. Anal. Appl., 276/2:845-876, 2002. 
[13] A. Buffa and R. Hiptmair. Galerkin boundary element methods for electromagnetic scattering. Computational Methods in Wave Propagation, 31:83-124, 2003.

[14] A. Bulovyatov. A parallel multigrid method for band structure computation of $3 D$ photonic crystals with higher order finite elements. PhD thesis, Karlsruhe Institute of Technology, 2010.

[15] M. Cessenat. Mathematical Methods in Electromagnetism (Series on Advances in Mathematics for Applied Sciences). World Scientific, Singapore, 1996.

[16] P. Ciarlet. The Finite Element Method for Elliptic Problems. North-Holland, Amsterdam, 1978.

[17] D.M. Copeland. Boundary-element-based finite element methods for Helmholtz and Maxwell equations on general polyhedral meshes. International Journal of Applied Mathematics and Computational Sciences, 2009.

[18] M. Costabel. Boundary integral operators on Lipschitz domains: Elementary results. SIAM J. Math. Anal, 19:613-626, 1988.

[19] R. Dautray and J.L. Lions. Mathematical Analysis and Numerical Methods for Science and Technology. Springer, Berlin, 1990.

[20] L. Debnath and P. Mikusiński. Introduction to Hilbert Spaces with Applications. Academic Press, San Diego, third edition, 2005.

[21] J.W. Demmel. Applied Numerical Linear Algebra. SIAM, 1997.

[22] K. Eriksson, D. Estep, P. Hansbo, and C. Johnson. Computational Differential Equations. Studentlitteratur, Lund, 1996.

[23] L.C. Evans. Partial Differential Equations. American Mathematical Society, 2010.

[24] Q. Fang. Complex Analysis. Peking University Press, 1996.

[25] G.B. Folland. Introduction to Partial Differential Equations. Princeton University Press, Princeton, NJ, second edition, 1995.

[26] G.B. Folland. Real Analysis. Wiley, 1999.

[27] G.B. Folland. Fourier Analysis and It's Application. American Mathematical Society, 2009.

[28] V. Girault and P. Raviart. Finite Element Methods for Navier-Stokes Equations. Springer, Berlin, 1986.

[29] P. Grisvard. Elliptic Problems in Nonsmooth Domains. Pitman, Boston, 1985.

[30] S. Guo. Electrodynamics. Higher Education Press, Beijing, 1997. 
[31] S. Hildebrandt. Über die Lösung nichtlinear Eigenwertaufgaben mit dem Galerkinverfahren. Math. Z., 101:255-264, 1967.

[32] R. Hiptmair. Symmetric coupling for eddy current problems. SIAM J. Numer. Anal., 40:41-65, 2002.

[33] R. Hiptmair and C. Schwab. Natural boundary element methods for the electric field integral equation on polyhedra. SIAM J. Number. Anal., 40:66-86, 2002.

[34] G.C. Hsiao and W.L. Wendland. Boundary element methods: Foundation and error analysis. Encyclopedia of Computational Mechanics, 1:339-373, 2004.

[35] G.C. Hsiao and W.L. Wendland. Boundary Integral Equations. Springer-Verlag, Berlin, Heidelberg, 2008.

[36] K. Huang. Solid Physics. Peking University Press, Beijing, 2009.

[37] J.D. Jackson. Classical Electrodynamics. John Wiley \& Sons, Inc., third edition, 1998.

[38] J.D. Joannopoulos, S.G. Johnson, J.N. Winn, and R.D. Meade. Photonic Crystals: Molding the Flow of Light. Princeton University Press, 2008.

[39] F. John. Partial Differential Equations. Springer-Verlag, New York, fourth edition, 1991.

[40] S. John. Strong localization of photons in certain disordered dielectric superlattices. Physical Review Letters, 58:2486-2489, 1987.

[41] O. Karma. Approximation in eigenvalue problems for holomorphic Fredholm operator functions i. Numerical Functional Analysis and Optimization, 17(3-4):365$387,1996$.

[42] O. Karma. Approximation in eigenvalue problems for holomorphic Fredholm operator functions ii (convergence rate). Numerical Functional Analysis and Optimization, 17(3-4):389-408, 1996.

[43] T. Kato. Perturbation Theory for Linear Operators. Springer-Verlag, Berlin, 1995.

[44] C.T. Kelley. Iterative Methods for Linear and Nonlinear Equations. Society for Industrial Mathematics, 1987.

[45] V.E. Laine. Photonic Crystals: Fabrication, Band Structure and Applications. Nova Science Publishers Inc, 2011.

[46] U. Langer, G. Of, O. Steinbach, and W.Zulehner. Inexact data-sparse boundary element tearing and interconnecting methods. SIAM J. Sci. Comput., 29:290âĂŞ314, 2007.

[47] S. Larsson and V. Thomee. Partial Differential Equations with Numerical Methods. Springer-Verlag, Berlin, Heidelberg, 2009. 
[48] W. McLean. Strongly Elliptic Systems and Boundary Integral Equations. Cambridge University Press, 2000.

[49] SG. Mikhlin. Mathematical Physics, an Advanced Course. North Holland, Amsterdam, 1970.

[50] P. Monk. Finite Element Methods for Maxwell's Equations. Oxford University Press, Oxford, 2003.

[51] J. Nédélec. Integral equations with non integrable kernels. Int. Eq. Operator Th., 5:562-572, 1982.

[52] J. Nédélec. Acoustic and Electromagnic Equations Integral Representation for Harmonic Problems. Springer, New York, 2001.

[53] O.Steinbach and M.Windisch. Stable boundary element domain decomposition methods for the Helmholtz equation. preprint, 2009.

[54] P.S. Pacheco. A User's Guide to MPI. Department of Mathematics, University of San Francisco, 1998.

[55] L. Paquet. Mixed problems for the Maxwell system. Annales de la Faculté des Sciences de Toulouse, 4(2):103-141, 1982.

[56] J.S. Przemieniecki. Matrix structural analysis of substructures. AIAA J., 1:138-147, 1963.

[57] J.S. Przemieniecki. Theory of Matrix Structural Analysis. MCGraw-Hill, New York, 1968.

[58] S. Rjasanow and O. Steinbach. The Fast Solution of Boundary Integral Equations. Springer Science+Business Media, New York, 2007.

[59] W. Rudin. Functional Analysis. McGraw-Hill Inc., New York, second edition, 1991.

[60] W. Rudin. Real and Complex Analysis. McGraw-Hill Education(Asia) Co. and China Machine Press, Beijing, third edition, 2004.

[61] S. Sahni. Data Structures, Algorithms, and Applications in C++. Silicon Press, 2004.

[62] S.A. Sauter and C. Schwab. Boundary Element Methods. Springer-Verlag, Berlin, Heidelberg, 2011.

[63] H.A. Schwarz. Vierteljahrsschrift der Naturforschenden Gesellschaft in Zürich, volume 15, pages 272-286. Springer, 1870.

[64] H.A. Schwarz. Gesammelte Mathematische Abhandlungen, volume 2, pages 133134. Springer, 1890. 
[65] O. Steinbach. Numerical Approximation Methods for Elliptic Boundary Value Problems. Finite and Boundary Elements. Springer Science+Business Media, New York, 2008.

[66] O. Steinbach and G. Unger. A boundary element method for the Dirichlet eigenvalue problem of the Laplace operator. Numer. Math., 113:281-298, 2009.

[67] O. Steinbach and G. Unger. Convergence analysis of a Galerkin boundary element method for the Dirichlet Laplacian eigenvalue problem. Technical report, Johann Radon Institute for Computational and Applied Mathematics, 2010.

[68] A. Toselli and O. Widlund. Domain Decomposition Methods - Algorithms and Theory. Springer, Berlin, 2005.

[69] O. Steinbach U. Langer. Boundary element tearing and interconnecting methods. Computing, 71:205-228, 2003.

[70] O. Steinbach U. Langer. Coupled boundary and finite element tearing and interconnecting methods in: Proceedings of the 15th international conference on domain decomposition. Lecture Notes in Computational Sciences and Engineering, Springer, Heidelberg, 40:83-97, 2004.

[71] G. Unger. Analysis of Boundary Element Methods for Laplacian Eigenvalue Problems. PhD thesis, TU Graz, 2009.

[72] H.A. Vorst. Computational Methods for Large Eigenvalue Problems (Lecture Notes). Mathematical Institute, Utrecht University, 2000.

[73] P. Wesseling. An Introduction to Multigrid Methods. R.T. Edwards, Inc., 2004.

[74] C. Wieners. Parallele Finite Elemente (Lecture Notes). Department of Mathematics, Karlsruhe Institute of Technology, 2004.

[75] C. Wieners. Distributed point objects: A new concept for parallel finite elements applied to a geomechanical problem. Future Generation Computer Systems, 22(4):532-545, 2006.

[76] C. Wieners. A geometric data structure for parallel finite elements and the application to multigrid methods with block smoothing. Journal Computing and Visualization in Science, 13(4):161-175, 2010.

[77] E. Yablonovitch. Inhibited spontaneous emission in solid-state physics and electronics. Physical Review Letters, 58:2059-2062, 1987.

[78] Y. Yu. A Concise Course on Electrodynamics. Peking University Press, Beijing, 1999.

[79] M. Zhou. Real Analysis. Peking University Press, Beijing, 2008. 